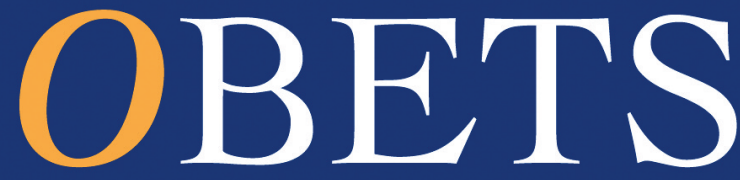

Revista de Ciencias Sociales

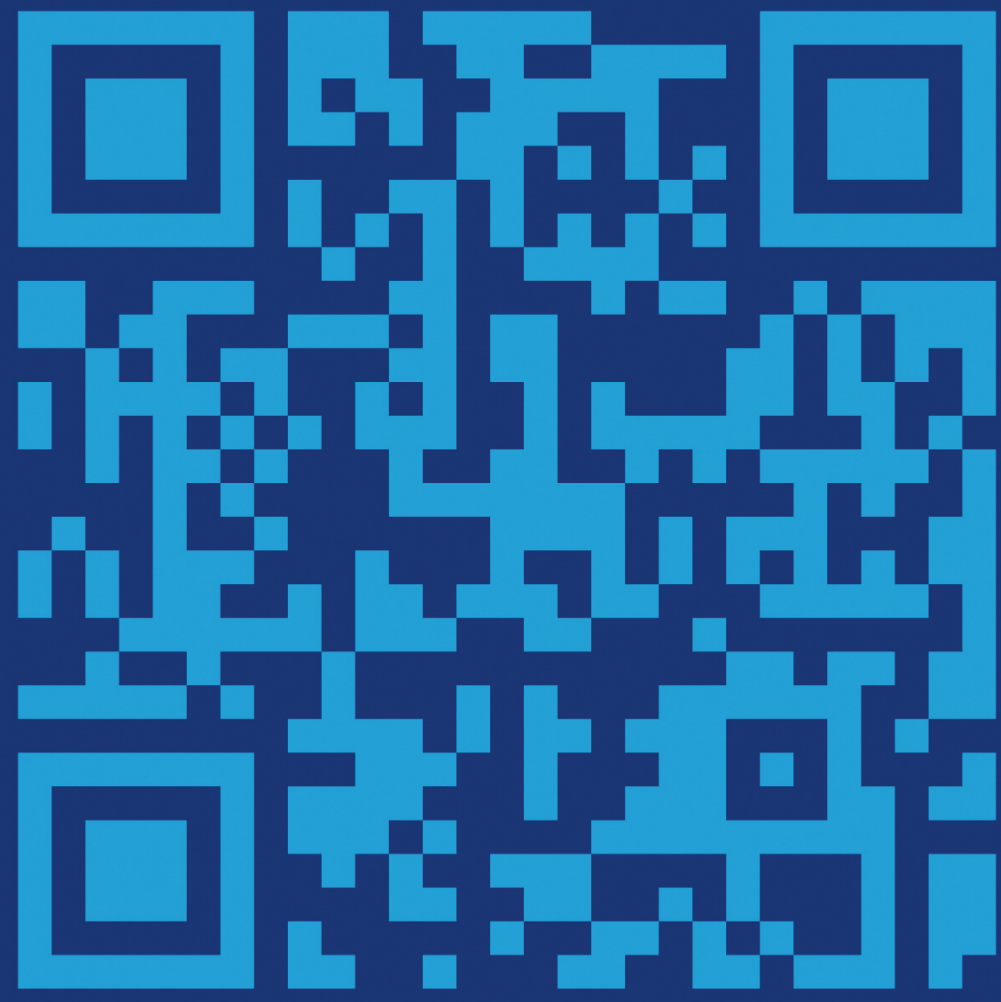

\title{
Ocio y desarrollo juvenil
}

\section{Noviembre 2017}

Instituto Interuniversitario de Desarrollo Social y Paz Universidad de Alicante

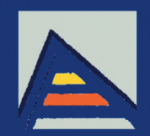

Universitat d'Alacant Universidad de Alicante 



\section{OBETS}

REVISTA DE CIENCIAS SOCIALES

PUBLICACIONES DE LA UNIVERSIDAD DE ALICANTE 
El presente número de la revista ha obtenido una ayuda del Vicerrectorado de Investigación, Desarrollo e Innovación de la Universidad de Alicante

Publicaciones de la Universidad de Alicante

Campus de San Vicente s/n

03690 San Vicente del Raspeig

Publicaciones@ua.es

http://publicaciones.ua.es

Teléfono: 965903480

Fax: 965909445

(C) de la presente edición: los autores

OBETS. Revista de Ciencias Sociales.

Editada por IUDESP.

Instituto Interuniversitario de Desarrollo Social y Paz.

Universidad de Alicante.

Periodicidad: Semestral

http://revistaobets.ua.es

Email: revistaobets@ua.es

ISSN: 2529-9727

e-ISSN: 1989-1385

Depósito legal: A-885-2008

DOI: $10.14198 /$ OBETS

Diseño de portada: candela ink. / O. Santacreu

Composición: Patricia Barbero

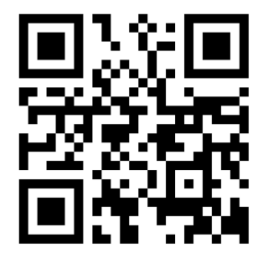

Los contenidos están bajo una licencia Creative Commons Reconocimiento 4.0 España.

Los contenidos pueden copiarse, distribuirse o comunicarse públicamente, bajo las siguientes condiciones generales: Reconocimiento. Debe reconocerse los créditos de la obra de la manera especificada por el autor o el licenciador (pero no de una manera que sugiera que tiene su apoyo o apoyan el uso que hace de su obra). Los términos de la licencia disponibles on-line en: http://creativecommons.org 
OBETS, Revista de Ciencias Sociales es una revista de investigación de periodicidad semestral editada por el Observatorio Europeo de Tendencias Sociales (OBETS), grupo de investigación integrado en la sede de la Universidad de Alicante (España) del Instituto Interuniversitario de Desarrollo Social y Paz (IUDESP). Dirigida a profesionales, investigadores/as, estudiantes, profesorado y público general interesado en la investigación académica en el campo de las Ciencias Sociales, comenzó a editarse en 2008.

La revista tiene como objetivo la difusión de estudios de naturaleza académica que contribuyan al mejor conocimiento de la sociedad contemporánea. En este sentido está abierta a la publicación de resultados de investigación empírica, metodológica o teórica de ámbito nacional e internacional. La revista abarca un amplio abanico de temas inscritos en el ámbito de la Sociología y Ciencias Sociales, como estudios de opinión pública, metodología, demografía, estructura y cambio social, ciencia política, teoría sociológica, antropología o psicología social. Los trabajos se publican en español, inglés, italiano, portugués o valenciano/catalán.

La revista está integrada en RUA, Repositorio Institucional de la Universidad de Alicante y cumple con el estándar de metadatos Dublin Core (http://www.dublincore.org) y con el protocolo OAI-PMH (Open Archives Initiative-Protocol for Metadata Harvesting: http://www.openarchives.org) para la transmisión de contenidos en Internet. Cuenta también con el DOI 10.14198/OBETS. A través de su página web y del RUA la revista ofrece opciones de acceso a contenido por títulos, autores, palabras claves o fecha.

A fin de alcanzar el mayor nivel de calidad posible en los trabajos publicados, todos los artículos se someten al arbitraje por pares académicos, considerando tanto la novedad de los resultados como el rigor metodológico en los diseños de investigación y análisis. El equipo editorial de OBETS. Revista de Ciencias Sociales no se hace responsable, en ningún caso, de la credibilidad y autenticidad de los trabajos. Del mismo modo, las opiniones y hechos expresados en cada artículo son de exclusiva responsabilidad de sus autores/as y OBETS. Revista de Ciencias Sociales no se identifica necesariamente con ellos. 
Incluida en:

\begin{tabular}{|l|l|}
\hline SCOPUS & $\begin{array}{l}\text { Base de datos bibliográfica de resúmenes y citas de artículos } \\
\text { de revistas científicas editada por Elsevier }\end{array}$ \\
\hline ESCI & $\begin{array}{l}\text { Emerging Sources Citation Index - Producto de la Web of } \\
\text { Science (WoS) editada por Thomson Reuters }\end{array}$ \\
\hline DICE & $\begin{array}{l}\text { Base de datos para la difusión y calidad editorial de las } \\
\text { revistas españolas de humanidades y ciencias sociales y } \\
\text { jurídicas }\end{array}$ \\
\hline ISOC & $\begin{array}{l}\text { ISOC - Base de datos del Centro de Información y } \\
\text { Documentación Científica (CINDOC) del CSIC }\end{array}$ \\
\hline CIRC & Clasificación Integrada de Revistas Científicas \\
\hline RESH & $\begin{array}{l}\text { Sistema de valoración integrada de revistas españolas de } \\
\text { Humanidades y Ciencias Sociales }\end{array}$ \\
\hline LATINDEX & $\begin{array}{l}\text { LATINDEX-Catálogo. Sistema regional de información en } \\
\text { línea para revistas científicas de América Latina, el Caribe, } \\
\text { España y Portugal }\end{array}$ \\
\hline DOAJ & DOAJ - Directory of Open Access Journals \\
\hline REDIB & $\begin{array}{l}\text { Red Iberoamericana de Innovación y Conocimiento } \\
\text { Científico }\end{array}$ \\
\hline DIALNET & $\begin{array}{l}\text { Portal de difusión de la producción científica iberoamericana } \\
\text { impulsado por la Universidad de La Rioja }\end{array}$ \\
\hline MIAR & MIAR: Matriu d'informació per a l'avaluació de revistes \\
\hline CiteFactor & CiteFactor: Academic Scientific Journals \\
\hline SJIF & SJIF Scientific Journal Impact Factor \\
\hline ULRICH'S & ULRICH'S Periodicals Directory \\
\hline RECOLECTA & $\begin{array}{l}\text { RECOLECTA: Recolector de Ciencia Abierta de la FECYT } \\
\text { y del Plan Estratégico REBIUN 2007-2010 }\end{array}$ \\
\hline SCIRUS & SCIRUS - Buscador de información científica \\
\hline SHERPA/RoMEO & $\begin{array}{l}\text { Políticas de copyright de las editoriales y autoarchivo. } \\
\text { University of Nottingham. }\end{array}$ \\
\hline DULCINEA & $\begin{array}{l}\text { Dulcinea - Proyecto coordinado por el Ministerio de } \\
\text { Educación y Ciencia para identificar y analizar las } \\
\text { políticas editoriales de las revistas científicas españolas }\end{array}$ \\
\hline
\end{tabular}




\section{EQUIPO EDITORIAL}

\section{Consejo de redacción}

\section{Dirección:}

Oscar Antonio Santacreu Fernández - IUDESP. Universidad de Alicante, España

\section{Secretaría:}

Francisco José Francés García - Departamento Sociología II. Universidad de Alicante, España

Liberto Carratalá Puertas. Departamento Sociología I. Universidad de Alicante, España

\section{Secretaría técnica:}

María Carmen Albert Guardiola - Unidad Técnica de Calidad. Universidad de Alicante, España

Vocales:

- Mercedes Alcañiz Moscardó - Universitat Jaume I de Castellón, España

- Cristino Barroso - Universidad de La Laguna, España

- Mario Alejandro Guillén García - Programa Interdisciplinario de Población y Desarrollo Local Sustentable (PYDLOS), Universidad de Cuenca, Ecuador

- Nina Rother - BAMF. Alemania

- Natalia Ix-Chel Vázquez González - Facultad de Ciencias Políticas y

Administración Pública. Universidad Autónoma del Estado de México, México

\section{Comité científico}

- Alberto Acosta Espinosa - Facultad Latinoamericana de Ciencias Sociales (FLACSO), Ecuador

- Antonio Alaminos Chica - IUDESP. Universidad de Alicante, España

- José Beltrán Llavador - Dpto. Sociología y Antropología Social, Universidad de Valencia, España

- Gianfranco Bettin - Università degli Studi di Firenze, Italia

- José Manuel Canales Aliende - Dpto. de Estudios Jurídicos del Estado, Universidad de Alicante, España

- Vidal Díaz de Rada - Universidad Pública de Navarra, España

- Guillermina Díaz Pérez - Universidad Autónoma del Estado de México

- Mariano Fernández Enguita - Universidad Complutense de Madrid, España

- Margarita Latiesa Rodríguez - Universidad de Granada, España

- María Asunción Martínez Román - Dpto. Trabajo Social y Servicios Sociales. Universidad de Alicante, España

- Beatriz Padilla - Instituto Universitário de Lisboa (ISCTE-IUL), Lisboa, Portugal

- Andrea Pirni - University of Genova, Italia

- Ettore Recchi - Università degli Studi 'G. d'Annunzio' Chieti e Pescara, Italia

- M'hammed Sabour - University of Eastern Finland, Finlandia

- Pedro Sánchez Vera - Universidad de Murcia, España

- Sylvie Strudel - Université Panthéon-Assas, Paris, Francia

- José María Tortosa Blasco - IUDESP. Universidad de Alicante, España

- Anna Triandafyllidou - European University Institute, Florencia, Italia; ELIAMEP, Atenas, Grecia 



\title{
OBETS \\ REVISTA DE CIENCIAS SOCIALES
}

\author{
MONOGRÁFICO
}

\section{OCIO Y DESARROLLO JUVENIL}

Coordinado por: Jaime Cuenca

y Aurora Madariaga 



\section{INDICE}

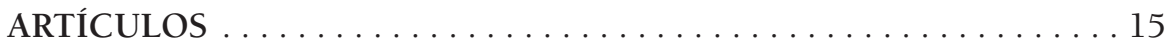

Jaime Cuenca, Aurora Madariaga

Ocio y desarrollo juvenil . . . . . . . . . . . . . . . . . . . . 17

Leisure and youth development

Fernando Bayón, Jaime Cuenca, José Antonio Caride

Reimaginar la ciudad. Prácticas de ocio juvenil y producción del espacio público urbano $\ldots \ldots \ldots \ldots \ldots \ldots \ldots \ldots \ldots \ldots \ldots . \ldots 21$

Reimagining the city. Youth leisure practices and the production of public urban space

María Belén Caballo Villar, Laura Varela Crespo, Eusebio Manuel Nájera Martínez

El ocio de los jóvenes en España. Una aproximación a sus prácticas y barreras .............................. 43

Leisure activities of young people in Spain. An approach to their practices and barriers

Nuria Codina, Jose V. Pestana, Robert A. Stebbins

Serious and casual leisure activities in the construction of young adult identity: a study based on participants' self-descriptions

Los ocios serio y casual en la construcción de la identidad juvenil.

Un estudio basado en la autoexpresión de los participantes

Joseba Doistua Nebreda, Yolanda Lázaro Fernández, Concepción Bru Ronda

La experiencia del ocio en familia de jóvenes estudiantes de enseñanza secundaria postobligatoria 81

Family leisure-time experiences of post-compulsory secondary education students 
Idurre Lazcano, Aurora Madariaga, Douglas A. Kleiber, Daniel Muriel

Satisfacción de las personas jóvenes con las actividades de ocio entre pares

Satisfaction of young people with leisure activities between peers

María Isabel Luis Rico, Tamara de la Torre Cruz, Alfredo Jiménez Palmero

Emprendimiento y ocio en la formación inicial del profesorado

de Educación Primaria . . . . . . . . . . . . . . . . . . . . . . . 121

Entrepreneurship and leisure in the initial of Primary Education teachers

Amaia Makua Biurrun, Macarena Cuenca-Amigo, Roberto San Salvador del Valle Doistua

Relación entre las prácticas de ocio significativas de la juventud y el emprendimiento social. El caso Think Big Jóvenes .......... 151 The relationship between youth's significant leisure practices and social entrepreneurship. The Think Big Jóvenes case

María Jesús Monteagudo, Ruth Ahedo, Ana Ponce de León

Los beneficios del ocio juvenil y su contribución al desarrollo humano ............................... 177 The benefits of youth leisure and their contribution to human development

Fátima Poza-Vilches, Ana Fernández-García, João Paulo Ferreira Delgado

Buenas prácticas de intervención social en relación al ocio con jóvenes en riesgo de exclusión desde la percepción de los agentes sociales 203

Good practices of social intervention in relation to leisure with young people at risk of exclusion from the perception of social agents

Magdalena Sáenz de Jubera Ocón, Eva Sanz Arazuri, Emauele Isidori Desarrollo personal mediante la actividad física. Percepción de los jóvenes españoles según áreas geográficas . . . . . . . . . . . . 229 Personal development through physical activity. Spanish youth's perception as a function of geographical areas 
Antonio Alaminos, Paloma Alaminos-Fernández

La potencia estadística prospectiva del voto retrospectivo en España (1996-2015)

The prospective statistical power of the retrospective vote in Spain (1996-2015)

CRÍTICA DE LIBROS 279

\section{Liberto Carratalá Puertas}

Francisco José Francés García, Antonio Alaminos Chica, Clemente Penalva Verdú y Óscar Antonio Santacreu Fernández La investigación participativa: métodos y técnicas . . . . . . . . . . . 281

NORMAS EDITORIALES E INSTRUCCIONES PARA COLABORACIONES ............................... 289

EDITORIAL STANDARDS AND INSTRUCTIONS FOR CONTRIBUTORS 

ARTículos ARTICLES 



\title{
OCIO Y DESARROLLO JUVENIL LEISURE AND YOUTH DEVELOPMENT
}

\author{
Jaime Cuenca \\ Instituto de Estudios de Ocio \\ Facultad de Ciencias Sociales y Humanas \\ Universidad de Deusto \\ jaime.cuenca@deusto.es \\ Aurora Madariaga \\ Instituto de Estudios de Ocio \\ Facultad de Ciencias Sociales y Humanas \\ Universidad de Deusto \\ aurora.madariaga@deusto.es
}

Como citar / Citation

Cuenca, Jaime y Madariaga, Aurora (2017) "Ocio y desarrollo juvenil".

OBETS. Revista de Ciencias Sociales, 12(Extra 1): pp-pp. 17-20.

doi:10.14198/OBETS2017.12.1.09

Este número monográfico recoge los datos de un amplio estudio en torno al ocio y el desarrollo juvenil a nivel estatal en el que hemos participado seis universidades españolas, realizado a partir de una muestra representativa con participantes de edades comprendidas entre los 16 y los 18 años. La investigación ha estado dirigida a conocer cuáles son las prácticas de ocio identificadas como más significativas por la población objeto de estudio, para poder correlacionarlas con datos relativos a la gobernanza de los espacios en que se desarrollan, la gestión de los tiempos que se les dedican, los beneficios psicosociales que los sujetos perciben derivarse de ellas, así como con información acerca de la compañía de referencia en cada uno de sus repertorios de actividades.

La muestra de alumnos ha sido completada con sendas muestras de profesores y padres, con objeto de introducir una mirada más compleja, que sirviera 
para integrar en el análisis las perspectivas de los educadores y las familias sobre las aportaciones reales de las experiencias de ocio al desarrollo social de los jóvenes españoles. El monográfico recoge los resultados contrastados de esta investigación transdisciplinar, en que expertos nacionales, en colaboración con investigadores europeos y estadounidenses de las ciencias sociales y humanas, la pedagogía y la psicología, han trabajado con una base de datos que les permite aproximarse de forma innovadora a las realidades y retos del fenómeno del ocio en jóvenes estudiantes de enseñanza secundaria post-obligatoria. Se ofrece así un complejo análisis de la construcción social y la gobernanza de los espacios emergentes en los que se localizan sus actividades recreativas, discutiendo la redefinición del papel de las familias en el disfrute intergeneracional de su tiempo libre e identificando nuevos estilos de vida latentes que reconsideran el peso que los roles de género siguen teniendo en su consolidación, todo lo cual hace de este monográfico, tanto en sus contenidos como en la consistencia metodológica de sus resultados, una aportación original, exhaustiva y actualizada a los estudios de ocio en nuestro país.

Los objetivos del monográfico son epistemológicos y sociopolíticos ya que, por una parte, queremos compartir con la comunidad científica el diseño de una herramienta válida para ponderar mejor la relevancia social del ocio en la constitución de la identidad juvenil, desde metodologías que concilian instrumentos cuantitativos de importante alcance estadístico (cuestionarios) con otros de naturaleza cualitativa (como entrevistas y focus group), cuya triangulación permite ofrecer una rica batería de datos al alcance de los investigadores interesados. Por otra parte, nos parece relevante sentar sobre esos datos las bases de una discusión de alcance sociopolítico, que nos permite reflexionar sobre asuntos tan relevantes como la correlación existente entre la construcción de la vida cotidiana y los consumos de tiempo educativo y social, las modalidades de supervivencia de las brechas de género a través de la repertorización de actividades recreativas, o las dificultades de equilibrar en el espacio público contemporáneo las formas institucionalizadas y autogestionadas de ese ocio que los jóvenes consideran precisamente como más libre, significativo y beneficioso.

Pretendemos contribuir a la literatura científica en castellano, dedicada al análisis de las prácticas de ocio juveniles, a la luz de las variables que definen el contexto social actual, así como brindar una panorámica científicamente sólida de esas variables y factores que están condicionando las múltiples realidades del ocio de los jóvenes españoles, entre ellas: las nuevas formas de gestión de los tiempos educativos y recreativos, el repertorio de aficiones significativas como medio de construcción de la identidad de los jóvenes y su inci- 
dencia en la redefinición de los roles de género, o la relación de los sujetos de este grupo de edad con el espacio público de convivencia, físico y virtual.

Consideramos necesario reflexionar sobre la emergencia de nuevos estilos de vida, en los que el ocio desempeña un papel configurador determinante. La inserción del joven en el espacio social está en gran medida apoyada sobre el repertorio de prácticas de ocio percibidas por él como más significativas. Este número recoge, con una extensión estadística notable, un mapa muy completo de los repertorios de actividades identificadas por los propios sujetos como más importantes en la conformación de su experiencia cotidiana. Al cruzar esta información con otras referidas a la frecuencia, la compañía, los lugares y los beneficios asociados a dichas actividades, es posible representar tendencias dominantes, susceptibles de ser interpretadas como inspiradoras de estilos de vida compatibles con y representativas de las circunstancias de nuestro presente socio-político.

Los trabajos aquí presentados aportan también luz sobre las modalidades más innovadoras de incorporación del joven a la vivencia comunitaria del espacio público, caracterizado por un creciente carácter híbrido, en la medida en que los jóvenes, nativos digitales, lo habitan y ocupan al mismo tiempo desde plataformas tecnológicas en red y en grupos físicos de pares afines: con sus riesgos y sus peligros, sus posibilidades y sus debilidades. El monográfico consta de diez artículos que profundizan en visiones complementarias de la importancia del ocio en el desarrollo juvenil. Los temas que se abordan son: la satisfacción de las personas jóvenes con las actividades de ocio; la vivencia del ocio en la articulación cotidiana de los tiempos sociales; el ocio serio en la construcción de la identidad; el ocio juvenil y la imaginación política del espacio; los beneficios del ocio juvenil; el desarrollo juvenil y la actividad física de ocio; la intervención social en el ocio con jóvenes en riesgo de exclusión; el emprendimiento y el ocio en la formación del profesorado de Educación Primaria; y la relación entre las prácticas de ocio significativas de la juventud y el emprendimiento social. Confiamos en que este amplio elenco de temas, atravesado por una preocupación unánime por el fenómeno del ocio como cauce de desarrollo juvenil, suscite interés en la comunidad científica y aliente nuevas direcciones y líneas de trabajo en la investigación.

JAIME CUENCA (Bilbao, 1983) es Doctor en Ciencias Sociales y Humanas y licenciado en Filosofía. Actualmente, es investigador asociado en el Instituto de Estudios de Ocio de la Universidad de Deusto y miembro del equipo de investigación reconocido Ocio y Desarrollo Humano. Ha impartido docencia en la 
Universidad de Deusto y en el Tecnológico de Monterrey en el ámbito de la teoría del ocio, estética, filosofía del arte y otras disciplinas filosóficas. Es autor de dos monografías y más de una veintena de artículos en revistas especializadas, tales como Arbor, World Leisure, Pensamiento, Journal of Leisure Research o Estudios filosóficos. Ha contribuido a varios catálogos de artista y ejerce como crítico de arte en diversos medios de prensa escrita.

AURORA MADARIAGA es doctora en Ocio. Máster en Educación Especial. Experto en Pedagogía Terapéutica. Psicóloga. Investigadora Principal del equipo A "El ocio como factor de desarrollo personal" (Gobierno Vasco, IT98416). Directora de la Cátedra Ocio y Discapacidad. Docente de grado y posgrado. Ha impartido multitud de cursos y ponencias. Además de artículos es coautora de varios libros sobre inclusión, accesibilidad, ocio y discapacidad. Ha dirigido tesis doctorales. Orcid. Org 0000-0002-8369-2781.

Recibido: 16/11/2016

Aceptado: 9/10/2017 


\title{
REIMAGINAR LA CIUDAD. PRÁCTICAS \\ DE OCIO JUVENIL Y PRODUCCIÓN DEL ESPACIO PÚBLICO URBANO ${ }^{1}$

\author{
REIMAGINING THE CITY. YOUTH LEISURE PRACTICES
} AND THE PRODUCTION OF PUBLIC URBAN SPACE
}

\author{
Fernando Bayón \\ Instituto de Estudios de Ocio \\ Facultad de Ciencias Sociales y Humanas \\ Universidad de Deusto, España \\ fernandobayon@deusto.es \\ Jaime Cuenca \\ Instituto de Estudios de Ocio \\ Facultad de Ciencias Sociales y Humanas \\ Universidad de Deusto, España \\ jaime.cuenca@deusto.es \\ José Antonio Caride \\ Facultad de Ciencias de la Educación \\ Universidad de Santiago de Compostela, España \\ joseantonio.caride@usc.es
}

Como citar / Citation

Bayón, Fernando; Cuenca, Jaime y Caride, José Antonio (2017) "Reimaginar

la ciudad. Prácticas de ocio juvenil y producción del espacio público urba-

\footnotetext{
${ }^{1}$ El trabajo que se presenta toma como referencia dos proyectos de investigación que, en el marco de dos convocatorias del Plan Nacional de I+D+i, han sido financiados por el Ministerio de Economía y Competitividad del Gobierno de España y por los Fondos FEDER de la Unión Europea: "De los tiempos educativos a los tiempos sociales: la construcción cotidiana de la condición juvenil en una sociedad de redes. Problemáticas específicas y alternativas pedagógico-sociales" (EDU2012-39080-C07-01 a 07_RESORTES) y "Educar el ocio: Realidades y perspectivas en clave intergeneracional, integral e inclusiva en una sociedad de redes" (EDU2015-65638-C6-1-R_ENREDOS).
} 
no". OBETS. Revista de Ciencias Sociales, 12(Extra 1): pp-pp. 21-41

doi:10.14198/OBETS2017.12.1.10

\section{Resumen}

Tras recorrer algunas de las recientes transformaciones discursivas en la comprensión del espacio urbano, este artículo teórico analiza tres prácticas de ocio juvenil, seleccionadas por su especial imbricación con la trama de la ciudad: el skateboarding, el graffiti y el juego de realidad aumentada Pokémon Go. Se pretende así contribuir a la comprensión del papel de las formas contemporáneas del ocio juvenil, y los tipos de experiencia territorial que les son propias, en la reestructuración del imaginario urbano y la producción del espacio público en la actualidad.

Palabras clave: ocio juvenil; espacio público; ciudad; imaginario.

\section{Abstract}

After reviewing some of the most recent discursive transformations in the understanding of urban space, this theoretical article analyses three youth leisure practices, which were selected because of their special link with the city plot: skateboarding, graffiti and the augmented reality game Pokémon Go. We intend to contribute to the understanding of the role of contemporary forms of youth leisure, and the their own types of experience, in the restructuring of the urban imaginary and the production of the public space nowadays.

Keywords: youth leisure; public space; city; imaginary.

\section{Extended abstract}

One of the most renowned analyses of critical urban geography, that of Henri Lefebvre (2013), assigned three specific characteristics to the space of the modern city: homogeneity, fragmentation and hierarchy. Even if his fundamental hypothesis, concerning the nature of space as a social product, remains unchallenged -or precisely because of that-, Lefebvre's description of urban space cannot simply continued to be applied to contemporary cities as it was when it was formulated back in the $60 \mathrm{~s}$ and $70 \mathrm{~s}$. The fabric and structure of modern urbanism was being contested then on the name of creativity and imagination by the Situationist International. But contemporary performative critiques to the ruling spatial principles of urban life need to come from different places and follow different strategies if the principles themselves have been thoroughly transformed. The strictly structured and rationalized city of Lefebvre has been replaced by the evanescent, flux-centered and -as suggested by Sloterdijk (2014)- foam-resembling postmetropolis of today.

One of the prevalent metaphors among urban development decision-makers and city branding managers is that of the city as an ever-changing scene always able to stage new and exciting events. In this light, leisure is not any more a free-time phenomenon giving functional meaning to clearly delimited areas of the urban plot. Rather, as a privileged arena for the late modern quest for intense lived experiences, leisure becomes a driving force in bringing forth new ways of understanding and living the city through embodied and socially meaningful action. This theoretical article intends to shed light on the role three 
specific youth leisure practices play in restructuring urban imaginaries and producing the public space of today: street skateboarding, graffiti and the augmented reality game Pokémon Go. Very different from each other in their history, the resources they require and the skills they help to develop, these three leisure practices though have something in common: they represent emplaced types of action that enact a creative and playful experience of the city. The selected youth leisure practices are analyzed in the article taking into account their own internal social logic and the language with which they imagine and appropriate public space. There are three main questions being addressed by our interpretation of the most relevant current scientific literature on these practices: first, how do they interact with urban space, understood as a social product; second, how do their participants re-imagine and re-signify the public space in the city; and third, how do they help define new functions and opportunities for the territory of the contemporary city, often encountering a hostile reaction while doing so.

Born as a youth leisure practice in southern California in the 50s, skateboarding was first located in found spaces (such as empty pools) and, later, in ad hoc built skateparks. It was only in the 80s that skaters began to choose downtown locations and use urban furniture for their 'tricks', hence marking the birth of street skateboarding. It encountered a degree of hostility among the general public not matched by other outdoor urban sport activities, which most probably relies on its condition of unstructured expressive physical activity, not linked to an external reward (Beal \& Weidman, 2003). Thanks to this peculiar type of activity, skaters develop their own imaginary around and a specific experience of the urban environment they use as a playground, thus embodying a performative critique of the city (Borden, 1998a, 2001a, 2001b; Woolley \& Johns, 2001).

The contemporary graffiti scene first developed in New York in the 70s, along other expressions of Afro-American hip hop youth culture, such as rap or breakdance. What remains specific about graffiti, as a visual creative practice, is that it cannot be understood without taking seriously its territorial condition. In order to achieve the sought after reputation, any graffiti writer (as graffiti artists are known) needs to balance the display of aesthetic skills with an extensive presence -mainly of his or her signature (tag)- throughout the city. Hence writers imagine the city as a space full of possibilities to assure peer recognition through an exercise of creative self-expression: the city is understood, not as a homogeneous unit, but as a continuous plane of multiple surfaces and interfaces (Halsey \& Young, 2006). Such a comprehension of urban space implies a neutralization of the marks and signs that intend to order it, a performative critique of officially sanctioned hierarchies of use and function, which explains the crusades against graffiti so many city councils around the globe have embarked on.

In July 2016, the American software development company Niantic launched Pokémon Go, an augmented reality location based game for iOS and Android mobile devices. Pokémon Go was one of the most popular apps of 2016 worldwide and by February 2017 its number of downloads amounted to 650 million, which makes it one of the youth leisure practices of greatest 
global impact in the last years. The hybrid nature of the game, consisting of the superimposition of physical and digital environments, allows the player to enjoy a specific experience of urban space: by adding digital elements, trivial and familiar locations become meaningful game loci, scenes of a ludic adventure through the city (Hjorth y Richardson, 2017). This combination of familiarity and excitement has a powerful effect on the players, many of whom change their daily routines because of the game, engaging in behaviour easily ridiculed by the media.

After analysing the three selected forms of contemporary youth leisure some commonalities become apparent. In their practice, the skater, the graffiti writer and the Pokémon Go player gain access to very specific experiences of urban environment and enact a lived space that is beyond some of the most fundamental features of modern urbanism, such as functional zoning and strictly delimited forms of land ownership. This kind of space is not totally unrelated to the most prevalent metaphors of the contemporary city; only, it does not rise from the abstract field of city branding, but from embodied and emplaced action in the living urban plot. That is what makes this three leisure practices plausible strategies to retake and reimagine public space in late modern cities.

\section{INTRODUCCIÓN}

La convicción de que se puede escrutar la génesis de la sociedad actual a través del espacio producido, se remonta al menos a los trabajos de Henri Lefebvre. En su ensayo filosóficamente más ambicioso, La producción del espacio, dejaba constancia de que el espacio de la modernidad posee tres caracteres precisos, que inicialmente pueden parecer incluso antagónicos: homogeneidad, fragmentación y jerarquización. Los modelos de gestión, así como las exigencias de vigilancia y comunicación, del espacio tienden a volverlo homogéneo en todas sus partes. Sin embargo, este espacio homogéneo se fragmenta en lotes, se deshilvana en bloques o parcelas, que acaban produciendo clausuras y guetos, pseudo-conjuntos pobremente vinculados con los centros urbanos. Además, esta fragmentación obedece a una estricta jerarquización: espacios residenciales, espacios comerciales, espacios de ocio, espacios marginales, etc. (Lefebvre, 2013: 58)

Este esquema del espacio moderno, cuyas raíces se hunden al menos en el París de Napoléon III, pero cuya paternidad más reciente suele atribuirse comúnmente a quienes, como la escuela Bauhaus o Le Corbusier, fueron primero tildados de revolucionarios para acabar posteriormente adoptados por el capitalismo avanzado, con su lógica de homogeneidad-fragmentación-jerarquización, puede que dé muestras de fatiga si recurrimos a él para explicar el comportamiento del espacio social contemporáneo. Pese a todo, la caracteriza- 
ción de Lefebvre sigue vigente en muchos de sus puntos clave: la homogeneidad del espacio oculta los conflictos reales, situación que el sociólogo francés cree extensible al funcionamiento del saber y la cultura, y aun de toda la sociedad, tras la Modernidad. Su hipótesis fundamental, la concepción del espacio como producto social, choca todavía hoy contra dos ilusiones. La primera es la ilusión de la transparencia, según la cual el espacio aparece como algo inteligible, un campo libre a la acción. Como alguien que circula cotidianamente por una región que disimula perfectamente sus peligros y oculta sus trampas, el ciudadano no ve detrás del diseño ningún designio. La segunda es la ilusión realista, que tiende a naturalizar ese artificio social llamado espacio urbano. La masiva aceptación de la facticidad del espacio, como un dato que se da por sentado dentro de lo que Blumenberg denominara "el mundo de la vida" (Blumenberg, 1999: 51), parece dejar claro hasta qué punto ha triunfado el rasgo general de la producción en el capitalismo, subrayado por la tradición marxista que va de Walter Benjamin a Henri Lefebvre: en los productos, sea un objeto o un espacio -o un espacio como objeto- se borran las huellas de la actividad productora tanto como es posible (Lefebvre, 2013: 255).

La ley del espacio urbano de la modernidad, tan poderosamente escrutada por Lefebvre, conoce sin embargo en nuestros días adendas múltiples e impugnaciones profundas. La organización del espacio público ha estado inspirada tradicionalmente por una cierta antropología social que repartía la urbe en zonas de accesibilidad normal, zonas de frontera -que imponían vedas relativas o absolutas a los flujos-, zonas residenciales y puntos de sutura que servían principalmente como lugares de encuentro e intercambio. Esta distribución ha padecido todo tipo de reformulaciones a lo largo de la historia; pero, con todo, ha mantenido su funcionalidad y, lo que acaso haya resultado más importante, ha normalizado un tipo de "civilidad" cuyo principal blasón ético era el de saber estar en el momento preciso en el espacio oportuno haciendo algo útil o aceptable que consintiera con la razón de ser del lugar.

Esta disciplina de producción del espacio urbano generó muy pronto contestaciones de diversa naturaleza, que coincidían en la pretensión de evidenciar el carácter restrictivo y anti-imaginativo de la ciudad burguesa del capitalismo avanzado. Seguramente una de las contestaciones más subversivas en ese sentido fue la protagonizada por la Internacional Situacionista que liderara a mediados de los años cincuenta Guy Debord quien, tanto en su Introducción a una crítica de la geografía urbana como especialmente en Teoría de la deriva, defiende un modo de comportamiento experimental ligado a las condiciones de la sociedad urbana, inspirado en técnicas de paso fugaz a través de ambientes aleatorios y heterogéneos, cuyos antecedentes habría que buscarlos en el 
surrealismo (Fargue: 2014; Aragon: 2016), inspirador también del flâneur benjaminiano (Benjamin, 2005). La ciudad ya no es un damero sobre el que deslizarnos ordenadamente, igual que las fichas de un juego regulado preliminarmente por agencias de autoridad indiscutida, sino un espacio discontinuo de puntos y vórtices que constituye una cartografía modulada por el estado de conciencia de cada uno de los que penetran en ella mezclando su exposición a los designios del azar con la intensificación de comportamientos afectivos hacia el medio físico (AAVV, 2006; Andreotti y Costa, 1996; Careri, 2013: 78-79). Pues las ciudades han transformado la anatomía de sus espacios de modo que, como afirma Peter Sloterdijk en el cierre de su trilogía "Esferas":

Se definen, por una parte, como emplazamientos de colectores pensados para colectivos reunibles; alojan, por otra, los complejos de apartamentos que sirven de cápsulas-vivienda a familias pequeñas o a quienes viven solos; $y$, finalmente, albergan las numerosas instalaciones del mundo del trabajo, en las que la mayoría de los habitantes de las ciudades aseguran sus bases económicas de existencia. Para la tarea de conformar un espacio común sobre los tres polos de la vida ciudadana (trabajo, vivienda, espacio público y colector) se han impuesto en la literatura urbanística las expresiones de tráfico y comunicación; como si se quisiera reducir el fenómeno ciudad a las generalidades del cambio de lugar y del flujo de datos. Desde que el impulso electrónico ha alcanzado a la teoría, esto llega hasta ficciones como la de la ciudad virtual, el territorio-online, la City of Bits, la Ciberville y metáforas de descorporeización semejantes. (Sloterdijk, 2014: 497)

Estamos, por lo tanto, ante un urbanismo-e que parece dejar muy atrás el concepto de "deriva" de Guy Debord: cuando el modelo de urbanismo vigente vaporiza la ciudad contemporánea en nombre de un nuevo romanticismo de la inmaterialidad que volatiliza las viejas zonificaciones de la cartografía urbana tardoburguesa (trabajo/residencia/ocio), y sustituye las localizaciones físicas por tramas casi fantasmales de nodos telemáticos (disembedding), todas las modalidades del flâneur y los mitos liberadores asociados al vagabundeo y la errancia parecen de repente trasnochados esfuerzos por reventar los límites físicos de lo que no cabe ya imaginar sino como una macro-espuma. Y precisamente estos cambiantes modos de imaginar la ciudad deben escrutarse con la mayor atención, porque el espacio no es algo dado, sino algo imaginado.

Asistimos, como habitantes de las ciudades, a una lucha diaria por la territorialización del espacio público urbano. Las modernas urbes apuestan cada vez más por la versatilidad de sus espacios, en la confianza de que, de este modo, sus calles y plazas puedan parecerse idealmente a un escenario acogedor de eventos múltiples. El espacio público urbano tiene que acreditar, como toda la fuerza de trabajo del capitalismo de mercado, su perenne y atlética disponi- 
bilidad a ser usada. Estas políticas mercadotécnicas, urgidas por el llamado city-branding que, según se insiste, "pone a las ciudades en el mapa", parecen apoyarse en el éxito de la metáfora del escenario, olvidando -o no- que el escenarium era la plataforma elevada dentro de un teatro que se reservaba para las representaciones... y de la que quedaba excluido, por definición, el público (Bayón, 2012: 410). En esta conversión de la ciudad en escenario de todo tipo de eventos (deportivos, culturales y lúdicos) desempeña un papel crucial el amplio repertorio de ofertas de ocio de las sociedades de consumo contemporáneas. El ocio no representa ya hoy, como en el urbanismo moderno, un conjunto de ocupaciones del tiempo libre al que se consagran funcionalmente ciertas áreas que, junto a las residenciales y las del trabajo, estructuran la trama urbana en una tríada de tanques estancos. Al contrario, como cauce privilegiado para canalizar la constante exigencia tardomoderna de vivencias excitantes en lo cotidiano (Cuenca, 2012), el ocio se revela en la actualidad como un agente extraordinario de reestructuración del imaginario urbano y la producción del espacio público. Es llamativo constatar cómo la volatilización del espacio real bajo las oportunidades masivamente brindadas por la digitalización se ha visto acompañada, desde el ámbito del ocio, por una forma lúdica de experiencia urbana hiperreal. Por ejemplo, la que facilitaban simuladores de ingeniería social, como el clásico videojuego Simcity, en cuyo manual de usuario se podía leer:

Cuando juegas a Simcity, tú diseñas, construyes y gestionas ciudades. (...) Eres el responsable. Si tu ciudad es un agradable para vivir, tu población crecerá. Si no lo es, tus Sims dejarán la ciudad. Y estate seguro de que te harán saber lo que piensan de ti y tus políticas (...). Uno de los retos más duros de Simcity 2000 es mantener una enorme ciudad sin sacrificar la calidad de vida de tus Sims, sin arruinarte por mantener las infraestructuras y sin subir los impuestos tanto que los negocios se deslocalicen (Soja, 2008: 473).

El producto más evolucionado de esta saga de simuladores fue Simcity Societies, lanzado en 2007, en que el usuario se investía con los poderes, no ya de un gestor urbanista a escala municipal, sino con los de un primer ministro. Este género de sustituciones hiperrealistas de la realidad, por otros medios, han acabado poblando la ciudad de espacios simbólicos que simulan un tema en particular o un paquete de paisajes-imágenes. Autores como Edward Soja han abordado de manera muy perspicaz esta Disneyización o "parque-tematización" del espacio público urbano de lo que denomina la "postmetrópolis" contemporánea; espacios cuyas comunidades pueden elegir entre visitar "Fantasilandia, Aventurelandia, Fronteralandia, La Tierra del Mañana o El Pueblo de los Dibujos Animados" (Soja, 2008: 474), cada uno con su imaginario ad hoc y su colección de regulaciones formales e informales. 
Con todo, hay prácticas de ocio contemporáneas constitutivamente híbridas y con un carácter marcadamente juvenil -tanto por el perfil de sus practicantes como por su contribución al actual ideal de juventud (Cuenca, 2013)-, que ayudan a entender que cualquier metaforización de la ciudad (la ciudadescenario, tanto como la ciudad-lienzo o la ciudad-interfaz) tiene otras derivas y no todas son proclives a un grado de institucionalización como el descrito hasta aquí. La producción de un espacio público con una creciente tendencia a vaporizarse en nombre de la conectividad telemática, el Internet de las cosas (Smart-cities), la versatilidad funcional y la "eventualización" digna de su nuevo estatuto de escenario, no debe llevarnos al error de pasar por alto la cotidiana pugna por recuperar territorios de la ciudad mediante intervenciones y prácticas que basculan entre lo deportivo, lo cultural y lo lúdico. En este artículo seleccionaremos, precisamente, una práctica de ocio juvenil por cada uno de estos tres ámbitos: el street skateboarding, el graffiti y el juego de realidad aumentada Pokémon Go.

Se trata de tres prácticas muy heterogéneas en cuanto a origen, trayectoria, recursos, hábitos o destrezas, pero todas ellas tienen en común algunos rasgos que van a estar presentes en nuestro análisis. Interactúan con el espacio fáctico proyectando sobre él itinerarios que obedecen a reglas prácticas y necesidades recreativas ni previstas ni amparadas por las funciones originalmente asignadas al mismo. Son formas de reescritura del espacio, suponen formas de reterritorialización que, más que a la necesidad neotribal de visibilizar el control simbólico que una determinada subcultura ejerce en un determinado espacio urbano (Maffesoli, 1990), expresan alternativas imaginativas a la creciente incorporeidad y estandarización de nuestros paisajes metropolitanos, poniendo de relieve formas de leer y corresponsabilizarse con el entorno que, sin ser punibles, parecen marginales por estar situadas fuera del consenso funcional asumido por el ethos mayoritario de los ciudadanos. Son, en fin, marcas territoriales que reestructuran el hábitat urbano conforme a itinerarios de prácticas que rozan con los usos previstos y consensuados en torno a los espacios de las ciudades. A la luz del análisis de estas prácticas pretendemos ofrecer una respuesta a la cuestión por el papel de las formas contemporáneas del ocio juvenil en la reestructuración del imaginario urbano y la producción del espacio público en la actualidad.

\section{METODOLOGÍA}

Tras habernos detenido, con carácter general, en algunas de las dinámicas urbanas más recientes, que ofrecen contextos siempre polémicos a la experiencia de la ciudad, la metodología que empleamos pretende analizar cada una de las prácticas seleccionadas desde sus propias lógicas sociales internas, atendien- 
do a los lenguajes con que ellas imaginan, planean y se apropian de sus interactuaciones con el espacio público. Estos son algunas de las variables que pretendemos explorar transversalmente: primero, cómo prácticas que se constituyen en manifestaciones específicamente urbanas del deporte al aire libre, la creatividad plástica y la "gamificación", interactúan con un espacio entendido como producto social; segundo, cómo dichas interacciones contribuyen a imaginar reestructuraciones del ecosistema urbano, conforme a códigos de conducta y lenguaje de los que emergen nuevos sentidos de pertenencia a la ciudad, a la vez que re-significan el espacio público heredado; y, tercero, cómo esas prácticas -del deporte, la plástica o el videojuego "urbanizados"-, no sólo son proyectos alternativos de cartografía de la ciudad, sino que se constituyen en espacios (vividos) dentro del espacio (recibido), con lo que ayudan a definir funciones y oportunidades del territorio conforme a lógicas que están -o al menos nacen- en los márgenes de esa versatilidad de nuestras metrópolis hoy ya políticamente institucionalizada y que a menudo encuentran una respuesta de larvada o explícita hostilidad.

Tabla 1. Esquema de las categorías de interpretación

\begin{tabular}{l|l|l|l}
\hline Ámbito del ocio & Tipo de práctica & Metáforas de la ciudad & $\begin{array}{l}\text { Reestructuración } \\
\text { del imaginario urbano }\end{array}$ \\
\hline Deportivo & Skate & La ciudad-escenario & $\begin{array}{l}\text { Movimiento: destrezas } \\
\text { que vencen sobre los } \\
\text { usos previstos del } \\
\text { mobiliario urbano }\end{array}$ \\
\hline $\begin{array}{l}\text { Creativo } \\
\text { (plástico) }\end{array}$ & Grafitti & La ciudad-lienzo & $\begin{array}{l}\text { Lenguaje: la plasticidad } \\
\text { marginal sobre soportes } \\
\text { estructurales y de } \\
\text { delimitación }\end{array}$ \\
\hline Lúdico & Pokémon-Go & La ciudad-interfaz & $\begin{array}{l}\text { Logro: recorridos físicos } \\
\text { que se hibridan y } \\
\text { justifican mediante } \\
\text { motivos digitalmente } \\
\text { diseñados }\end{array}$ \\
\hline
\end{tabular}

\section{RESULTADOS Y DISCUSIÓN}

\section{Street skateboarding: el uso imprevisto del escenario urbano}

Surgido en el sur de California a lo largo de los años 50 como práctica de ocio juvenil, el skateboarding conoció su primera popularización a comienzos de la 
década siguiente, cuando la industria de tablas de surf comenzó a fabricar skates para su comercialización masiva (Beal, 2013; Borden, 1998a). En un primer momento, el skateboarding fue concebido por sus practicantes y comunicado públicamente como una extensión del surf en tierra firme, una forma de sidewalk surfing (surf de acera). En esta primera escena californiana, el stakeboarding comenzó a practicarse en espacios encontrados, especialmente propicios para la imitación de ciertas maniobras del surf (como piscinas vacías), y no fue sino hasta avanzados los años 70 cuando aparecieron los primeros skateparks, entornos construidos ad hoc para facilitar ejercicios de creciente dificultad acrobática (Borden, 1998a). A finales de los 80 surge propiamente el street skateboarding, que abandona las pistas de los skateparks para ocupar el centro mismo de las ciudades, aprovechando el mobiliario urbano para sus rutinas exhibitorias (Beal, 2013, pp. 19-21).

Como el resto de variedades, el street skateboarding se estructura alrededor de los trucos (tricks): un vasto repertorio de maniobras cuyo progresivo dominio va marcando la destreza del practicante. En lo que constituye todo un patrimonio comunitario de los skaters a nivel global, los trucos son movimientos cuidadosamente codificados, sometidos a unas exhaustivas nomenclatura (nollie, pop, FS pop, etc.) y tipología (trucos de trip, de grab, old school, etc.) y en ocasiones incluso vinculados al recuerdo colectivo de su inventor (como es el caso del ollie, creado por Alan "Ollie" Gelfand en los 70). Cada skater se ve obligado a repetir pacientemente un cierto número de movimientos hasta que domina el truco correspondiente. Aun cuando esto se ejecute en comunidad, a menudo por turnos y ante la mirada de otros practicantes, cada skater compite sobre todo consigo mismo; en un skate spot (lugar de reunión de skaters) se encontrará antes un acicate a la emulación que un verdadero sentido de competencia. Las actitudes patentemente competitivas son juzgadas con severidad: es el desarrollo de un estilo propio de autoexpresión creativa lo que se valora (Beal y Weidman, 2003).

La peculiaridad del street skateboarding consiste en que esta búsqueda colectiva de la destreza individual tiene lugar en el espacio público de la ciudad. Aun cuando cualquier paraje urbano podría concebirse como un skate spot en potencia, pueden identificarse una serie de características que concurren indefectiblemente en los lugares más exitosos entre los practicantes (Woolley y Johns, 2001). En primer lugar, el espacio debe ser fácilmente accesible desde otras partes de la ciudad, por lo que a menudo se localiza en ubicaciones céntricas, cercanas a nodos de la red viaria y del transporte público. En segundo lugar, la disposición del terreno y del mobiliario urbano debe ofrecer las condiciones adecuadas para el aprendizaje y la ejecución de trucos (por ejemplo, 
por medio de la presencia de escalones, barandillas o rampas). En tercer lugar, debe facilitar, al mismo tiempo, la sociabilidad entre practicantes, lo que a menudo implica que quienes están descansando puedan contemplar los ejercicios y exhibiciones de los demás desde los márgenes. Por último, los skaters suelen elegir espacios cuyo uso sea compatible con el tránsito de peatones, al menos en ciertos horarios (Woolley y Johns, 2001, pp. 214-215).

Esta última prevención no evita que el street skateboarding se perciba como más amenazador, en términos generales, que otras actividades deportivas en el espacio público, vistas bajo una luz más positiva (Monteagudo y Morejón, 2016), lo que parece deberse a su demanda de una acción física no estructurada de antemano, con altas dosis de improvisación y sin la subordinación a ninguna meta externa predeterminada (Beal y Weidman, 2003, p. 344). Son precisamente estas características de su práctica las que conducen a los skaters a desarrollar un peculiar imaginario en torno al espacio urbano que usan como territorio lúdico (Borden, 1998a, 2001a, 2001b; Woolley y Johns, 2001). Este uso sólo es posible porque arraiga en el espacio moderno descrito por Lefebvre: un espacio homogéneamente pavimentado, tendente al plano y la línea recta, cuya fragmentación en una serie de parcelas funcionales jerarquizadas favorece una notoria diversidad de elementos divisorios y de mobiliario urbano. A un tiempo, el street skateboarding surge de este tipo de entorno urbano y se rebela contra él, asignándole una serie de usos y significados imprevistos que subvierten las funciones oficialmente sancionadas y las someten a una "crítica performativa" (Borden, 2001b). Un elemento de seguridad, por ejemplo, como la barandilla de unas escaleras, se convierte para el skater en un estímulo para maniobras exhibitorias de alto riesgo. Este ejercicio de reasignación creativa de significados explica la potencia desestabilizadora del skateboarding:

The meaning of the skateboard move then in part takes its power and vitality from the fact that it comes out-of-the-blue, an unexpected and sudden eruption of meaning where society had previously been content to say nothing. Skateboarding is a critique of the emptiness of meaning (Borden, 2001b: 187).

Cabe ver en esta capacidad de resignificar el espacio cotidiano en entornos delimitados de acción lúdica un reverso de la "parque-tematización" de la que habla Soja (2008), aquí desligada de sus compromisos con los intereses hegemónicos de la postmetrópolis contemporánea. De hecho, esa vocación de subvertir los sentidos del espacio público ha llevado a que el street skateboarding sea percibido con frecuencia como una amenaza por los agentes administrativos y económicos de la ciudad, que no dudan en tomar medidas para impedir o al menos limitar su práctica en muchos centros metropolitanos alrededor 
del mundo y tratar de salvaguardar así la solidez de las funciones y significados atribuidos a la trama urbana (Németh, 2006). Tales medidas van desde la imposición de sanciones económicas cuantiosas hasta la intervención física sobre el espacio urbano mediante la adición de pinchos a las barandillas o la instalación de pavimentos rugosos o de gravilla (Borden, 1998b).

\section{Graffiti: la ciudad como lienzo inagotable}

La escena contemporánea del graffiti surge en el Nueva York de los años 70 en el marco de la cultura juvenil afroamericana del hip-hop, junto a otros elementos característicos como el rap o el breakdance. Frente a anteriores manifestaciones gráficas en los muros urbanos (tan antiguas como la ciudad misma), el graffiti se rige por unas reglas muy características por las cuales el practicante (writer, escritor), para ganarse el respeto de sus pares, debe lograr la máxima visibilidad en un entorno hostil a su trabajo y que siempre está dispuesto, por tanto, a eliminarlo del espacio público (Macdonald, 2001). Esto lleva a buscar la colocación de piezas muy elaboradas y de gran calidad estética en lugares especialmente visibles por su importancia funcional en la trama de tránsitos urbanos (como los vagones de metro, por ejemplo) o cuyo difícil acceso asegure la permanencia (como la estructura de un acueducto). Pero, al mismo tiempo, obliga a compensar esa tarea de elevado riesgo y alta inversión de tiempo y recursos con otra actividad menos comprometida: el tagging, es decir, la proliferación del propio tag (o firma) por toda la ciudad (Macdonald, 2001; Taylor, 2011). El tag de un escritor es un signo idiosincrático que debe poder realizarse rápidamente en cualquier lugar, a menudo con un rotulador grueso. Sólo desarrollando una marca reconocible por la comunidad del graffiti puede un escritor ir completando su proceso de "dejarse ver" (getting up) e ir creándose una reputación. La especificidad del graffiti como práctica de ocio juvenil se malinterpretaría si se concibiera centrada exclusivamente en el resultado estético de cada pieza: su dimensión territorial goza de igual importancia (si no superior).

Pese a que las prácticas visibilizadoras del control simbólico del entorno, identificadas por Maffesoli (1990) en su exploración del neotribalismo tardomoderno, constituyen el más amplio contexto de la dimensión espacial del graffiti, esta se ha hecho acreedora, frente a otras, de un tratamiento particularmente alarmista o condescendiente por parte de la literatura científica. Por un lado, desde los años 70 no han dejado de sucederse las interpretaciones que han procurado subsumir el fenómeno en los usos de marcado territorial entre grupos rivales de adolescentes potencialmente inclinados a una trayectoria delictiva (Ley y Cybriwsky, 1974; Taylor, 2011); por otro lado, se ha conceptua- 
lizado la búsqueda de visibilidad del escritor a través del tagging como una práctica adictiva y cercana a la psicopatología (Pani y Sagliaschi, 2009; Taylor, 2012). Halsey y Young (2006) argumentan que este tipo de lecturas, y otras equivalentes en términos de clase o género, dificultan cualquier aproximación honesta al modo en que los propios escritores viven y se explican su práctica, algo que hacen predominantemente en términos de placer y orgullo. La consideración de la práctica como fuente de placer, esencial para su comprensión como forma de ocio juvenil, debe comprenderse en toda su complejidad: hay una satisfacción emocional a la vista del desarrollo de destrezas específicas, una potente descarga de adrenalina derivada de la ilegalidad de la práctica y, sobre todo, una sensación de que se está haciendo "lo correcto" al intervenir sobre un muro (Halsey y Young, 2006). Esta última nota, quizá la más difícil de comprender desde fuera del mundo del graffiti, revela que los escritores experimentan el espacio urbano de un modo peculiar.

A uni-coloured wall is considered 'boring' -as 'negative space'- and therefore as something to be filled out or brought to life. (...) Most importantly, for many writers the surfaces that make up the built environment present more in the order of a flow than a structure. They are, in other words, canvasses permanently in waiting. (Halsey y Young, 2006: 288)

En términos generales, puede decirse que un escritor de graffiti imagina la ciudad como un espacio lleno de posibilidades para lograr el reconocimiento de sus pares a través de un ejercicio de auto-expresión creativa: la ciudad no aparece como un todo homogéneo organizado en diversas funciones jerarquizadas, sino como un plano continuo de múltiples superficies (Halsey y Young, 2006: 296). Los muros, que habitualmente cumplen una función delimitadora entre diversas formas y estatutos de propiedad del espacio, aquí se reinterpretan de un modo totalmente distinto. Frente a una concepción "ortogonal", por la cual el muro separa entre un adentro y un afuera, se instaura un uso "longitudinal" que conecta un aquí con un allí, como si cada pared fuera parte de una conversación vertical ilimitada entre pares (Brighenti, 2010: 329). Esta comprensión del espacio urbano implica una neutralización de todos los signos y marcas que lo ordenan habitualmente, una crítica-desde los márgenes- de las jerarquías de uso y función oficialmente sancionadas. Como afirma el antropólogo Néstor García Canclini:

Las luchas por el control del espacio se establecen a través de marcas propias y modificaciones de los graffiti de otros. Sus referencias sexuales, políticas o estéticas son maneras de enunciar el modo de vida y de pensamiento de un grupo que no dispone de circuitos comerciales, políticos o massmediáticos para expresarse, pero que a través del graffiti afirma su estilo. Su trazo manual, 
espontáneo, se opone estructuralmente a las leyendas políticas o publicitarias "bien" pintadas e impresas, y desafía esos lenguajes institucionalizados cuando los altera. El graffiti afirma el territorio pero desestructura las colecciones de bienes materiales y simbólicos. (Canclini, 2001: 306)

De ahí que la actividad de los escritores de graffiti sea percibida, no sólo como un acto de vandalismo por el público general, sino como una auténtica amenaza por los actores que quieren preservar el monopolio en el poder de marcado y ordenación del espacio urbano. Desde los años 70 hasta la actualidad, las autoridades de la ciudad de Nueva York, cuna de esta práctica de ocio juvenil, han llevado a cabo una auténtica cruzada contra el graffiti (Dickinson, 2008; Kramer, 2010), imitada por muchas otras ciudades en otras partes del mundo. En última instancia, este tipo de campañas anti-graffiti se revelan solidarias de una concepción de la ciudad que privatiza el espacio público y criminaliza cualquier intervención sobre el mismo que no tenga finalidad comercial (Dickinson, 2008).

\section{Pokémon Go: jugando en la interfaz físico-digital}

En julio de 2016, la compañía estadounidense de desarrollo de software Niantic lanzó Pokémon Go, un juego de realidad aumentada geolocalizada para dispositivos móviles iOS y Android. El juego aprovechaba buena parte de la tecnología aplicada en un proyecto anterior de Niantic, denominado Ingress, pero, gracias a un acuerdo de colaboración con Nintendo, sacaba partido a la franquicia Pokémon, un vasto conjunto de personajes ficticios que han protagonizado videojuegos y series televisivas de animación desde los años 90. Fue probablemente esta elección de contenidos la que convirtió el lanzamiento de Niantic en todo un éxito a nivel global, acercando al gran público, por primera vez, un proyecto que combinaba realidad aumentada y geolocalización. Aunque el fenómeno fue disminuyendo en intensidad a medida que quedaba atrás el verano de 2016, Pokémon Go fue una de las aplicaciones móviles más populares del año y en febrero de 2017 superaba ya los 650 millones de descargas (Sarkar, 2017). Las primeras estimaciones calculaban que el 70\% de los usuarios de la aplicación eran menores de 35 años, lo que la convierte en uno de las prácticas de ocio juvenil de los últimos años con mayor impacto a nivel global (YouGov, 2016).

El objetivo del juego consiste en capturar todos los Pokémon posibles y hacerlos evolucionar. Para conseguirlo, el jugador debe desplazarse físicamente por su entorno cercano, el cual visualiza en forma de mapa en su dispositivo móvil. Cuando se encuentra en la cercanía de un Pokémon, puede cambiar a la vista de realidad aumentada, en la que el personaje digital se le aparece superpuesto a la imagen del entorno físico, captada por la cámara del disposi- 
tivo móvil. Determinados puntos geolocalizados son relevantes para el avance en el juego: las Poképaradas, donde el usuario puede encontrar objetos necesarios para capturar y hacer evolucionar Pokémon, y los gimnasios, donde los jugadores pueden enfrentar a sus Pokémon entre sí. Ambos tipos de ubicaciones están localizadas, por lo general, en puntos de interés del espacio público, tales como monumentos, parques o fuentes, aunque a lo largo de 2016 compañías como McDonald's o Starbucks también llegaron a acuerdos para colocar Poképaradas y gimnasios en algunos de sus locales (Mochizuki, 2016; Starbucks, 2016). Desde el comienzo, el tratamiento del fenómeno que hicieron los medios de comunicación se centró principalmente en la ubicación -en ocasiones polémica- de estos puntos geolocalizados y en los diversos problemas ocasionados por la afluencia de usuarios del juego a los mismos (que en ciertos casos llegó a ser ciertamente masiva).

Buena parte de la creciente literatura científica sobre Pokémon Go se ocupa también de sus posibles impactos negativos y riesgos asociados. Tan pronto como en agosto de 2016, un artículo publicado en PARIPEX. Indian Journal of Research, exponía las fases que podían conducir al jugador a un comportamiento compulsivo y concluía con estas alarmantes palabras: "Before the 'Pokemania' or 'Pokemon mania' drives individual crazy the sooner the consorted action is taken is the better" (Bhattacharyya, Ray, Bhattacharya y Mallick, 2016). Pero más allá de la jugabilidad, es la condición geolocalizada de la aplicación y, por tanto, el necesario tránsito de sus usuarios por el espacio urbano, lo que ha centrado la mayoría de los análisis. Así, por ejemplo, se ha alertado del riesgo de posibles traumatismos debidos a ceguera por falta de atención (Barbieri et al., 2017), así como del aumento de exposición de los jugadores a las enfermedades transmitidas por mosquitos (Oidtman et al., 2016). Por otro lado, también se han detectado posibles beneficios derivados del uso extensivo del espacio urbano al que se ven motivados los jugadores más comprometidos: se ha mostrado la influencia de Pokémon Go en el aumento de los niveles de actividad física (Alhoff, White y Horvitz, 2016; Nigg, Mateo y An, 2017; Wong, 2017) y se han señalado también sus posibles aportaciones a la conciencia de la protección de espacios naturales (Dorward, Mittermeier, Sandbrook y Spooner, 2017).

Con todo, no son numerosos los estudios que se ocupan en profundidad de la naturaleza híbrida del juego y analizan la peculiar experiencia del espacio urbano que facilita en el jugador la superposición entre el entorno físico y el digital. Como señalan Hjorth y Richardson (2017), la adición de elementos digitales permite que entornos banales y familiares se transformen en lugares de juego significativos. Es aquí donde reside la especificidad de Pokémon Go, 
que no ofrece un mero entretenimiento casual en los intervalos entre las ocupaciones cotidianas, sino que, más bien, interviene explícitamente sobre esas ocupaciones y rutinas y puede llegar a modificarlas (Hjorth y Richardson, 2017: 5). En efecto, un análisis geoestadístico a gran escala de diversos elementos del juego ha comprobado que Pokémon Go induce cambios en la movilidad de los jugadores. Estos parecen adaptar sus rutinas diarias para desplazarse a lugares cercanos a su domicilio o su lugar de trabajo donde puedan encontrar Pokémon y, además, tienden a moverse por la ciudad en pares o pequeños grupos con mayor frecuencia (Colley et al., 2017). Pero la naturaleza híbrida del juego no afecta sólo a las acciones de los jugadores en el espacio urbano, sino a su percepción, experiencia e imaginación del mismo. Dada la identidad que el juego presupone entre los movimientos del jugador por su entorno físico y los de su avatar en el espacio digital, no puede restringirse el significado del mapa mostrado en la pantalla del dispositivo móvil a un mero dispositivo representacional de orientación: por el contrario, debe entenderse como una compleja interfaz procesual que permite la navegación por una realidad híbrida (digital y analógica a un tiempo) -tal y como han mostrado Lammes y Wilmott (2011) para otros juegos basados en geolocalización-. Los puntos de interés del espacio digital se sobrescriben a los del entorno físico, y no se hacen notar con menor eficacia, ya sea a través de estímulos ópticos (realidad aumentada) o táctiles (el dispositivo Pokémon Go Plus, que consiste en una pulsera inteligente que vibra cuando un Pokémon se encuentra cerca). La peculiar condición híbrida de esta práctica de ocio juvenil la hace particularmente solidaria de las más recientes metáforas descorporeizadas del espacio urbano que, como señala Sloterdijk (2014), acaban subsumiendo la ciudad en una especie de imaginada macro-espuma de conexiones y tránsitos.

No obstante, el potencial de distorsión de las interacciones en el espacio público que acompaña a este tipo de experiencia territorial híbrida no debería desdeñarse. A lo largo del verano de 2016 se reportaron numerosos casos de comportamientos colectivos anómalos -como súbitas concentraciones de jugadores en busca de un Pokémon poco común (Chen, 2016)-, así como conductas individuales que se consideraron ofensivas -como la prosecución del juego en espacios muy cargados históricamente o con relevancia religiosa (Sarabia, 2016)-. En muchas ciudades del mundo, las autoridades no tardaron en tomar medidas prohibiendo el juego en áreas determinadas.

\section{CONCLUSIONES}

El skater que surfea por la trama material de la ciudad con un deslizamiento exhibitorio evadido de las necesidades de circulación, el escritor de graffiti que 
emplea la superficie de un edificio como bastidor de un lenguaje encriptado, el jugador que caza Pokémon virtuales en un entorno físico desde la interfaz de su smartphone: todos ellos reestructuran con sus prácticas el imaginario urbano, produciendo un espacio que es capaz de trascender algunos de los más fundamentales ordenamientos del urbanismo moderno, como son las zonificaciones funcionales y los estatutos de propiedad del suelo. Todos ellos "leen" de manera diversa el espacio. Crean una legibilidad alternativa de la ciudad. La legibilidad es de una importancia decisiva en el escenario urbano (Lynch, 2010:11): y esa legibilidad heterodoxa se produce en los bordes sobre los que se aplican preferentemente los skaters, cabalgando sobre los límites entre dos zonas de clases diferentes; en los nodos, focos estratégicos de la imagen de la ciudad, las confluencias que obligan al viandante a convertirse necesariamente en observador y a aguzar su atención, y que se convierten en la ubicación prominente del grafiti; o en los hitos, los puntos u objetos de referencia que se consideran exteriores a las personas y a los que casi todas las ciudades adhieren una historia para que así se refuerce su valor como mojón, hoy reinventados y reprogramados por Pokémon Go. Siguiendo los elementos constitutivos de la imagen de la ciudad estudiados en su día por Kevin Lynch, podría concluirse que, efectivamente, estas tres prácticas son otras tantas constelaciones desde las que se está reimaginando la ciudad. No hay en esto, quizá, como hemos tratado de mostrar, una diferencia que los oponga radicalmente a las últimas derivas en la metaforización de la ciudad contemporánea, cuyas instancias hegemónicas obstinadamente imaginan como dotada de infinita versatilidad, constituida por eventos y flujos, casi incorpórea. Ahora bien, el skater, el escritor de graffiti y el jugador de Pokémon Go no producen metáforas ad hoc desde el abstracto territorio del city branding, sino que ponen en práctica su imaginario urbano en una experiencia concreta, corpórea, incardinada en la trama viva de la ciudad. Es este anclaje el que convierte a estas tres formas del ocio juvenil contemporáneo en otras tantas estrategias posibles para recuperar y repoblar creativamente el espacio público de las ciudades tardomodernas.

Sería ingenuo, en cualquier caso, rodear estas prácticas de un aura utópica que prometiera un espacio urbano libre de contradicciones e inequidades. Se ha denunciado, por ejemplo, cómo el graffiti y el skateboarding canalizan formas de construcción de la masculinidad que invisibilizan el talento y la iniciativa de las mujeres participantes (Beal y Weidman, 2003; Macdonald, 2001), o cómo la distribución de Poképaradas refuerza las desigualdades entre vecindarios de diversa composición étnica (Colley et al., 2017). Tampoco puede ignorarse el origen corporativo de Pokémon Go que, frente a las otras prácti- 
cas, constituye en realidad un producto diseñado y distribuido por una compañía con afán de lucro. Con todo, estas consideraciones no pueden servir para anular la experiencia de los participantes ni para negar cualquier virtualidad a su impacto sobre los imaginarios urbanos. Aun en grados muy diversos, que van desde la persecución legislativa a la ridiculización mediática, estas tres prácticas de ocio juvenil han encontrado en el público general una reacción de mayor o menor hostilidad. Como afirma Dickinson (2008: 40) en referencia al graffiti, lo que anida en el fondo de estas polémicas es la cuestión sobre quién está autorizado a iniciar proyectos en el espacio público. Y a reimaginarlo por completo, añadimos.

\section{BIBIOGRAFÍA}

AA.VV. (2006). Urbanismo situacionista. Barcelona: Gustavo Gili.

Althoff, T., White, R.W. y Horvitz, E. (2016). Influence of Pokémon Go on Physical Activity: Study and Implications. Journal of Medical Internet Research, 18(12):e315, doi:10.2196/jmir.6759

Andreotti, L. y Costa, X. (Eds.). (1996). Teoría de la deriva y otros textos situacionistas sobre la ciudad. Barcelona: MACBA/Actar.

Aragon, L. (2016). El aldeano de París. Madrid: Errata Naturae.

Barbieri, S., Vettore, G., Pietrantonio, V., Snenghi, R., Tredese, A., Bergamini, M., Previato, S., Stefanati, A., Gaudio, R.M., Feltracco, P. (2017). Pedestrian Inattention Blindness While Playing Pokémon Go as an Emerging Health-Risk Behavior: A Case Report. Journal of Medical Internet Research, 19(4):e86. doi:10.2196/jmir.6596

Bayón, F. (2012). Las retóricas del público. El espacio de consumo del arte como institución política. Arbor. Ciencia, Pensamiento y Cultura, 188 (754), 409-426.

Beal, B. (2013). Skateboarding. The Ultimate Guide. Santa Barbara, CA: Greenwood.

Beal, B. y Weidman, L. (2003). Authenticity in the Skateboarding World. En R.E. Rinehart y S. Sydnor (Eds.), To the Extreme: Alternative Sports Inside and Out (pp. 337-352). Albany, NY: State University of New York Press.

Benjamin, W. (2005). Libro de los pasajes. Madrid: Akal.

Bhattacharyya, R., Ray, U., Bhattacharya, J., y Mallick, B. (2016). Pokemania, Threats and Concerns of A Simple Augmented Reality Game. PARIPEX-Indian Journal of Research, 5(8). 363-366.

Blumenberg, H. (1995). Naufragio con espectador. Paradigma de una metáfora de la existencia. Madrid: Visor.

Borden, I. (1998a). Body architecture: skateboarding and the creation of super-architectural space. En J. Hill (Ed.), Occupying Architecture: Between the Architect and the User (pp. 111-125). London: Routledge.

Borden, I. (1998b). An affirmation of urban life: Skateboarding and socio-spatial censorship in the late twentieth century city. Archis, 5, 46-51. 
Borden, I. (2001a). Skateboarding, Space and the City: Architecture and the Body. Oxford: Berg.

Borden, I. (2001b). Another Pavement, Another Beach: Skateboarding and the Performative Critique of Architecture. En I. Borden, J. Rendell, J. Kerr y A. Pivaro (Eds.), The Unknown City. Contesting Architecture and Social Space (pp. 178-199). Cambridge, MA / London: MIT Press.

Brighenti, A.M. (2010). At the Wall: Graffiti Writers, Urban Territoriality, and the Public Domain. Space and Culture. 13(3), 315-332.

Careri, F. (2013). Walkscapes. El andar como práctica estética. Barcelona: Gustavo Gili.

Chen, J. (16 de julio de 2016) Pokemon Go: Crowd Stampedes Through Central Park After Rare Vaporeon Sighting. US Magazine. Recuperado de http://www.usmaga zine.com/celebrity-news/news/pokemon-go-crowd-stampedes-after-spottingrare-pokemon-w429525

Colley, A., Thebault-Spieker, J., Lin, A. Y., Degraen, D., Fischman, B., Häkkilä, J. y Wenig, D. (2017). The Geography of Pokémon GO: Beneficial and Problematic Effects on Places and Movement. In Proceedings of the SIGCHI Conference on Human Factors in Computing Systems. doi:10.1145/3025453.3025495

Cuenca, J. (2012). Génesis de la comprensión experiencial del ocio en la modernidad tardía: transformaciones del concepto de vivencia. Arbor. Ciencia, Pensamiento y Cultura, 188(754), 315-325, doi:10.3989/arbor.2012.754n2005

Cuenca, J. (2013). Peter Pan disecado. Mutaciones políticas de la edad. Bilbao: consonni.

Debord, G. (2004). Introducción a una crítica de la geografía urbana. A Parte Rei: revista de filosofía, 11, 10-13.

Debord, G. (2010).Teoría de la deriva. Revista Anthropos: Huellas del conocimiento, 229, 197-200.

Dickinson, M. (2008). The Making of Space, Race and Place New York City's War on Graffiti, 1970 - the Present. Critique of Anthropology, 28(1), 27-45.

Dorward, L. J., Mittermeier, J. C., Sandbrook, C. y Spooner, F. (2017). Pokémon Go: Benefits, Costs, and Lessons for the Conservation Movement. Conservation Letters, 10, 160-165. doi:10.1111/conl.12326

Fargue, L.P. (2014). El peatón de París. Madrid: Errata Naturae.

García Canclini, N. (2001). Identidades híbridas. Estrategias para entrar y salir de la modernidad. Barcelona: Paidós.

Halsey, M. y Young, A. (2016). Our desires are ungovernable. Theoretical Criminology, 10(3), 275-306.

Hjorth, L., y Richardson, I. (2017). Pokémon GO: Mobile media play, place-making, and the digital wayfarer. Mobile Media y Communication 5(1), 3-14, doi: 10.1177/ 2050157916680015

Kramer, R. (2010). Moral panics and urban growth machines: official reactions to graffiti in New York City, 1990-2005. Qualitative Sociology, 33(3), 297-311.

Lefebvre, H. (2013). La producción del espacio. Madrid: Capitán Swing.

Ley, D. and Cybriwsky, R. (1974). Urban graffiti as territorial markers. Annals of the Association of American Geographers, 64(4), 491-505. 
Macdonald, N. (2001). The Graffiti Subculture: Youth, Masculinity and Identity in London and New York. Hampshire, UK / New York, NY: Palgrave Macmillan.

Maffesoli, M. (1990). El tiempo de las tribus. Barcelona: Icaria.

Mochizuki, T. (19 de julio de 2016). McDonald's Unit to Sponsor Pokémon Go in Japan. Wall Street Journal. Recuperado de https://www.wsj.com/amp/articles/ mcdonalds-unit-to-sponsor-pokemon-go-in-japan-1468936459

Monteagudo Sánchez, M. J., y Morejón Torné, S. (2016). Ocio deportivo en los waterfronts de Bilbao y Barcelona. Un estudio comparativo. Revista de Psicología del Deporte, 25(supl. 2), 15-20.

Nigg, C.R., Mateo, D.J., y An, J. (2017). Pokémon GO May Increase Physical Activity and Decrease Sedentary Behaviors. American Journal of Public Health, 107(1), 3738, doi:10.2105/AJPH.2016.303532

Oidtman, R.J., Christofferson, R.C., ten Bosch, Q.A., Espana, G., Kraemer, M.U.G., Tatem, A., Barker, Ch.M. y Perkins, T.A. (2016). Pokémon Go and Exposure to Mosquito-Borne Diseases: How Not to Catch 'Em All. PLoS Currents, 8, doi: 10.1371 /currents.outbreaks.2d885b05c7e06a9f72e4656d56b043cd

Pani, R. y Sagliaschi, S. (2009). Psychopathology of Excitatory and Compulsive Aspects of Vandalistic Graffiti. Psychological Reports, 105(3_suppl), 1027-1038.

Sarabia, D. (6 de septiembre de 2016). Un youtuber detenido en Rusia por jugar a Pokémon Go en una iglesia. Eldiario.es. Recuperado de http://www.eldiario.es/cul tura/tecnologia/Entrenador-juegues-Pokemon-Go-iglesia_0_556094518.html

Sarkar, S. (27 de febrero de 2017). Pokémon Go hits 650 million downloads. Polygon. Recuperado de https://www.polygon.com/2017/2/27/14753570/pokemon-godownloads-650-million

Sloterdijk, P. (2014). Esferas III: Espumas. Madrid: Siruela.

Soja, E.W. (2008). Postmetrópolis. Estudios críticos sobre las ciudades y las regiones. Madrid: Traficantes de sueños.

Starbucks (8 de diciembre de 2016). "Discover the Pokémon GO Frappuccino at Starbucks". Starbucks.com. Recuperado de https://news.starbucks.com/news/starbuckspokemon-go

Taylor, M. F. (2012). Addicted to the Risk, Recognition and Respect that the Graffiti Lifestyle Provides: Towards an Understanding of the Reasons for Graffiti Engagement. International Journal of Mental Health and Addiction, 10(1), 54-68.

Taylor, M.F. (2011). Hanging with the Hoodies: Towards an Understanding of the Territorial Tagging Practices of Prolific Graffiti Writers Seeking an Adolescent Nonconforming Social Identity. International Journal of Child and Adolescent Health, 4(3), 223-232.

Wong, F. Y. (2017). Influence of Pokémon Go on physical activity levels of university players: a cross-sectional study. International Journal of Health Geographics, 16(1), 8.

Wooley, H. y Johns, R. (2001). Skateboarding: The City as a Playground. Journal of Urban Design, 6(2), 211-230. 
YouGov (2016). Pokémon Go: 34m downloads, 31m current players, and 10m paying users since U.S. launch. YouGov.com. Recuperado de https://today.yougov.com/news/ 2016/08/12/pokemon-go-34m-downloads-31m-current-players-and-1/

FERNANDO BAYÓN (Bilbao, 1971) es Doctor en Filosofía por la Universidad de Deusto (UD). Actualmente, es Investigador de la Línea "Ocio y desarrollo humano" del Instituto de Estudios de Ocio de la Facultad de Ciencias Sociales y Humanas de UD, en la que es también profesor de "Historia de Filosofía en la Modernidad: Idealismo y Dialéctica", dentro del grado de "Filosofía, Política y Economía". Es autor y/o editor de más de cincuenta publicaciones científicas dedicadas a la construcción narrativa de la memoria en el espacio político europeo. Entre sus libros, se cuenta: "Filosofía y Leyenda: Variaciones sobre la última modernidad (de Tolstói a Musil)" (Barcelona, Antrhopos, 2009). orcid.org/0000-0002-4465-8643

JAIME CUENCA (Bilbao, 1983) es Doctor en Ciencias Sociales y Humanas y licenciado en Filosofía. Actualmente, es investigador asociado en el Instituto de Estudios de Ocio de la Universidad de Deusto y miembro del equipo de investigación reconocido Ocio y Desarrollo Humano. Ha impartido docencia en la Universidad de Deusto y en el Tecnológico de Monterrey en el ámbito de la teoría del ocio, estética, filosofía del arte y otras disciplinas filosóficas. Es autor de dos monografías y más de una veintena de artículos en revistas especializadas, tales como Arbor, World Leisure, Pensamiento, Journal of Leisure Research o Estudios filosóficos. Ha contribuido a varios catálogos de artista y ejerce como crítico de arte en diversos medios de prensa escrita.

JOSÉ ANTONIO CARIDE es Catedrático de Pedagogía Social en la Facultad de Ciencias de la Educación de la Universidad de Santiago de Compostela, en la que es el Comisionado de su "Campus da Cidadanía" en las áreas de Ciencias Sociales y Jurídicas, Artes y Humanidades. Dirige el Grupo de Investigación "Pedagogía Social y Educación Ambiental" (SEPA-interea). Presidió la Sociedad Iberoamericana de Pedagogía Social (SIPS), entre 2002 y 2013. En 2004 le fue concedida la "Orden al Mérito Institucional" del World Council For Curriculum and Instruction. Sus publicaciones y líneas de investigación pueden consultarse en: https://usc-es.academia.edu/Jos\%C3\%A9AntonioCaride. Código orcid: orcid.org/0000-0002-8651-4859 



\title{
EL OCIO DE LOS JÓVENES EN ESPAÑA. UNA APROXIMACIÓN A SUS PRÁCTICAS Y BARRERAS \\ LEISURE ACTIVITIES OF YOUNG PEOPLE IN SPAIN. AN APPROACH TO THEIR PRACTICES AND BARRIERS
}

\author{
María Belén Caballo Villar \\ Departamento de Pedagogía y Didáctica, Grupo de investigación SEPA-interea \\ Facultad de Ciencias de la Educación \\ Universidad de Santiago de Compostela, España \\ belen.caballo@usc.es \\ Laura Varela Crespo \\ Departamento de Pedagogía y Didáctica, Grupo de investigación SEPA-interea \\ Facultad de Ciencias de la Educación \\ Universidad de Santiago de Compostela, España \\ laura.varela@usc.es \\ Eusebio Manuel Nájera Martínez \\ Instituto de Filosofía. Pontificia \\ Universidad Católica de Valparaíso, Chile \\ eusebio.najera@pucv.cl
}

\section{Como citar / Citation}

Caballo Villar, María Belén; Varela Crespo, Laura y Nájera Martínez, Eusebio Manuel (2017) "El ocio de los jóvenes en España. Una aproximación a sus prácticas y barreras". OBETS. Revista de Ciencias Sociales, 12(Extra 1): pp-pp. 43-64. doi:10.14198/OBETS2017.12.1.11

\section{Resumen}

El artículo toma como referencia el proyecto "De los tiempos educativos a los tiempos sociales: la construcción cotidiana de la condición juvenil en una sociedad de redes. Problemáticas y alternativas pedagógico-sociales" (EDU2012-39080C07). Presenta los resultados referidos a la utilización del tiempo libre derivados de un cuestionario aplicado en el curso 2015-2016 a una muestra de 
2694 estudiantes de Educación Secundaria Postobligatoria. Se concluye el predominio de prácticas asociadas a las dimensiones lúdica, cultural y festiva del ocio, siendo residual la dimensión solidaria. Destacan las barreras temporales y el déficit de alfabetización en ocio.

Palabras clave: ocio; juventud; barreras de ocio; dimensiones del ocio.

\section{Abstract}

The article takes the project "From educational times to social times: the daily construction of being young in a network society. Specific problems and socialpedagogical alternatives" (EDU2012-39080-C07). It presents the results relating to the use of free time arising from a questionnaire applied in the course for 2015-2016 to a sample of 2,694 students in Post-Compulsory Secondary Education. The predominance of practices associated with leisure, cultural and festive entertainment aspects, with the solidarity dimension being residual. Of particular note are temporary barriers and the literacy deficit in leisure activities.

Keywords: Leisure; youth; leisure barriers; leisure dimensions.

\section{Extended abstract}

The article is part of the coordinated project "From educational times to social times: the daily construction of being young in a network society. Specific problems and social-pedagogical alternatives" (EDU2012-39080-C07), focused on the study of the educational and social times of students who attend PostCompulsory Secondary Education in the Spanish context (16-18 years). Its objective is to understand and interpret the impact of school and social times in youth socialization processes, analysing how, and to what degree they influence the daily life of the boys and girls of different contexts and realities. This contribution focuses its arguments on the study of leisure activities that young people carry out in their free time, understanding that such practices are associated with values, needs, experiences, etc., which are essential for the construction of youth identities. Leisure that is claimed as a complex experience, and not as mere entertainment or cluster of activities to fill time. Leisure is pursued as a space for individual and community development, as well as an expression of quality of life and, consequently, as a right that no person should be deprived of. However, it is in this period of youth -continuing with what has already been experienced since childhood- when the tensions caused by the conflict between school, family and social times are experienced, given that far from a temporary harmonization that makes it possible to create opportunities of coexistence and participation in everyday life, the times of young people are placed in a scenario characterised by an exponential acceleration of their rhythms of life and their educational needs, limiting their possibilities for leisure activities in which they are interested. From this perspective, the barriers and educational, cultural, economic factors, etc., shall be examined which are limiting the ability and the opportunities that are available to boys and girls to participate in activities that they would like to do and which, for various reasons, they are not carrying out in their free time. 
A stratified probabilistic sampling was applied when choosing the sample using proportional allocation in each of the six areas in which the Spanish territory was divided, which has facilitated a manageable number of strata with a reasonably homogeneous behaviour in the interior and heterogeneous with respect to the other areas. The work has a margin of error of $1.9 \%$ and a confidence level of $95 \%$. Information was collected during the academic year 2015-2016 using a questionnaire prepared ad hoc, designed to capture the perceptions, needs, habits and expectations related to the distribution and uses of school, educational and social times of students in Post-Compulsory Secondary Education -Baccalaureate, Middle grade Vocational Training and PCPI (currently basic vocational training) - $(\mathrm{n}=2694)$. The application is carried out taking into account criteria of rurality and urbanity (minimum of one rural centre in each area, depending on the demographic characteristics), as well as the ownership of the centre (out of four selected schools, three were public and one private).

The data show that leisure practices of young people in Post-Compulsory Secondary Education are mainly concentrated in aspects of leisure (sport, digital leisure), creative (cultural activities) and festive, with their participation in solidarity initiatives being limited. Similarly, activities such as tourism and table games do not provide adolescents with a relevant leisure experience. The lack of civic involvement of young people in volunteer work or through the use of associations is worrying, given that these types of life experiences are fundamental for the construction of youth identity in a time that, being disinterestedly shared, is oriented towards the exercise of active citizenship.

With regard to the places where young people carry out their leisure activities, the presence of private spaces stands out, with the family home constituting a central place for the enjoyment of free time, not only with regards to digital leisure activities but also for carrying out other hobbies (listening to music, watching movies, etc.). Other scenarios such as shopping centres and the street also have a prominent presence in young peoples' leisure habits. It should also be noted that the school is not perceived by young people as a space to enjoy free time, the spending of the academic day there being experienced as tedious.

Together with economic factors, the lack of time constitutes one of the main barriers that limit the participation of young people in leisure activities that they like, highlighting their desire for more time during the week to get involved in initiatives that are not strictly academic. School time for adolescents and young people between the ages of 16 and 18 years plays a central role by being the focal point of all the activities they carry out, highlighting the time they must devote daily to doing academic tasks as a barrier to their leisure habits. From Monday to Friday, the feeling of lack of time is accentuated with that which refers to being with friends, the family or playing sports. In addition, the gender gap is present in the enjoyment of leisure experiences. Finally, the contributions of work are to identify habits in leisure trends of young people in Spain and the spaces in which they carry them out, showing 
some of the factors that limit their chances of undertaking valuable leisure experiences. In this sense, the need for education in entertainment is called for -barring contextual, economic, social constraints, etc.- so that boys and girls can acquire the knowledge, skills, attitudes and the confidence necessary to enable them to give a personal and positive meaning to these experiences. The set of knowledge, skills and interests that can be developed in disadvantaged contexts differs widely from the opportunities to be used in scenarios that are educationally enriching and promoters of a taste for learning. A question to which is added the persistence of gender barriers that limit opportunities for girls to participate in leisure activities that they would like to do.

As a result, it can be stated that leisure education as a process that extends throughout life, is essential in the stage of being young to ensure equal opportunities and resources in the access to quality leisure. The aim is not only to promote the development of individual skills, but also to draw attention to the need that community resources be created to ensure the conditions for a valuable leisure that empowers people and groups.

\section{INTRODUCCIÓN: DE LOS TIEMPOS DE OCIO COMO DERECHO EDUCABLE}

La condición juvenil se ha constituido en un tema relevante en las últimas décadas. Desde la investigación (González y Feixa, 2013; García Canclini, 2014), se reconoce que el saber sobre la juventud va transitando desde visiones esencialistas y universalistas hacia la constatación de una "condición juvenil heterogénea" que orienta los estudios hacia los fenómenos juveniles particulares articulados en la vida cotidiana y la construcción de subjetividades, al mismo tiempo que se significan como una metáfora de cambio social. Se habla de la presencia de nuevos modos de existencia, mediados por una revolución tecnológica que implica un cambio radical de paradigma (Reig y Vílchez, 2013) y se nos informa de una cuarta revolución industrial basada en la innovación y la creatividad. En este escenario, las formas de producción social, cultural, política y económica se enfocan al despliegue de sociedades de aprendizaje, donde se perfila al knowmad, nómade del conocimiento, como sujeto del siglo XXI (Cobo y Moravec, 2011).

Lo que los últimos estudios nos indican sobre las prácticas juveniles es (González y Feixa, 2013; Reig y Vílchez, 2013): 1) la existencia de generaciones que ya han nacido y se han desarrollado en los entornos tecnológicos actuales; 2) que tienen acceso cotidiano, en su hogar y en sus habitaciones, a diversos medios audiovisuales y digitales; 3 ) que han socializado, principalmente con sus pares, a través de estos medios, tanto para sus aprendizajes como en el desarrollo de sus relaciones personales y en diversos procesos de participación; 4) que han establecido modalidades de aprendizaje en contextos no reglados, 
generado la autoproducción simbólica y el emprendimiento colectivo temporal, flexible y precario. También existe en ellos un distanciamiento y una crítica de las instituciones, principalmente por la desconexión percibida en lo que se refiere a sus problemas cotidianos y por la visión negativa que la sociedad tiene de la juventud.

A diferencia de las generaciones anteriores, el manejo de redes con pares, el uso de móviles, la producción de información y comunicaciones a través de los medios audiovisuales, son algunas de las expresiones de la vida y cultura juvenil actual; expresiones mayoritariamente vinculadas al consumo, lugar desde el cual existe, desde hace décadas, un diálogo y una retroalimentación autónoma de las otras instituciones de socialización (familia y escuela). Empero, el seguimiento de las trayectorias juveniles ofrece hoy, según García Canclini (2014), una visión dinámica y crítica de estas tensiones, enfatizando el valor del acceso a los bienes simbólicos y las estrategias diferenciadas y alternativas que presentan las diversas experiencias juveniles. Para el autor, cada vez más jóvenes reclaman reconocimiento, espacios alternativos de sociabilidad y conectividad en los cuales satisfacer sus necesidades y producir identidad.

Además de la convivencia indisociable y cotidiana en los espacios físicos y virtuales, una de las variables que nos ayuda a situar y comprender las realidades de los jóvenes contemporáneos es el tiempo, los tiempos. Amparo Lasén (2000) publicaba, coincidiendo con el inicio del siglo XXI, un informe titulado A contratiempo: un estudio de las temporalidades juveniles; un libro que ayuda a comprender los tiempos de las personas jóvenes a través de sus discursos y prácticas, situándolas en un escenario social caracterizado por una exponencial aceleración no sólo de sus tiempos, sino también de sus ritmos de vida, de sus exigencias formativas, de su incertidumbre.

El tiempo siempre ha sido, pero cada vez lo es más, un elemento clave en la organización de las sociedades. Y de su correcta gestión pública, de las posibilidades individuales y sociales para armonizar los tiempos comunitarios, familiares y personales, los de trabajo y de ocio, depende buena parte de la calidad de vida y del bienestar. En el tiempo se crean las oportunidades de encuentro y convivencia, de participación en la vida social; en el tiempo se generan diálogos, intereses, expectativas... pero hace falta tenerlo y saber administrarlo. La juventud experimenta -y lo ha vivido ya en la infancia- las tensiones provocadas por los conflictos entre los tiempos escolares, los familiares y de ocio, lejos de la deseable armonización que reclamábamos. Las exigencias de los tiempos formativos $-\mathrm{y}$ sus prolongaciones en forma de tareas para casa-, sumado a las obligaciones y responsabilidades en el ámbito familiar, restan posibilidades y, en ocasiones, se imponen a la necesidad de tiempo libre y autónomo 
que pueda ser vivido como ocio. Sin embargo, para hacer referencia al ocio no es suficiente con tener tiempo libre, dado que éste ha de estar dotado de tres valores fundamentales: libertad, satisfacción y gratuidad; orientándose hacia referentes de identidad, superación y justicia (Cuenca, 2014).

Un ocio que, recogiendo las propuestas de la Carta Internacional de Educación del Ocio (WLRA, 1994), se reclama como experiencia compleja, y no como mero cúmulo de actividades para llenar el tiempo. Se reivindica como espacio de desarrollo individual y comunitario, como expresión de calidad de vida y, consecuentemente, como un derecho del que ninguna persona debe ser privada. En sus dimensiones se concretan los beneficios y potencialidades que las vivencias de ocio nos ofrecen, diferentes dependiendo de su encuadre en los ámbitos lúdico, festivo, creativo, solidario y ambiental-ecológico (Cuenca, 2011, 2014). Sin duda, son las dimensiones lúdica y festiva aquellas que el imaginario colectivo identifica como características de la juventud, relacionándose con el juego (reglado o espontáneo), el deporte, los hobbies... y que proporcionan descanso y diversión; o aquellas otras que suponen tiempo compartido, colectivo, la manifestación más social del ocio en la que se va creando identidad juvenil, especialmente en los grupos de pares.

Pero sin minusvalorar la importancia de estas expresiones del ocio, entendemos necesario potenciar en mayor medida aquellas que se concretan en las dimensiones creativa, solidaria y ambiental-ecológica, más complejas porque requieren de una educación del ocio no siempre accesible a la infancia y a la juventud que vive en contextos empobrecidos económica, social y/o culturalmente. Vivencias relacionadas, por ejemplo, con el disfrute o la práctica de la música, la danza, la literatura, el teatro o la pintura requieren aprendizaje y formación. Cuenca (2011) alude a la importancia de la creatividad y la reivindica en su sentido más amplio, situando la creación y la producción como elemento central de la experiencia de ocio, pero también su vertiente re-creativa, asociada al descubrimiento, comprensión y disfrute de la obra ajena.

Por su parte, la dimensión solidaria evoca acciones en beneficio de los demás de forma generosa y altruista, desinteresada, por el placer de compartir un tiempo y sentirse socialmente activo. Etxeberria y Segú (2011: 126-127) argumentan la necesidad de trabajar de forma holística el ocio solidario con los jóvenes, subrayando la interconexión de algunas de las dimensiones que estamos refiriendo: aluden al aspecto sostenible, que se concreta en el respeto por el patrimonio -fruto de la concienciación y sensibilización sobre el medio ambiente-, al aspecto inclusivo, que pone el acento en la aceptación, el respeto y el reconocimiento de la igualdad ante personas diferentes, y el aspecto comunitario, que se plasma en el trabajo con la comunidad, colaborando en 
redes que contribuyen a la justicia social. Estamos pues ante una dimensión del ocio con un gran potencial para impulsar el desarrollo de las personas y de los entornos que habitan.

Y son precisamente estos entornos, naturales y construidos, los que dan forma a la última de las dimensiones referidas, la ambiental ecológica, que pone el acento en el contexto o ambiente a la hora de motivar experiencias satisfactorias. Según Cuenca (2011) la vertiente ambiental alude al disfrute del ocio motivado, bien por la presencia de personas bien por la huella que han dejado en forma de patrimonio - de nuevo constatamos las intersecciones entre ámbitos de ocio-, mientras que la vertiente ecológica se asocia al disfrute de la naturaleza y evoca el concepto de ocio sostenible, en el que confluyen la educación del ocio y la educación ambiental.

Así pues, la educación del ocio, como proceso que nos acompaña a lo largo de la vida -pero que tiene un papel clave en la infancia y en la juventud-, resulta imprescindible para asegurar la igualdad de oportunidades y recursos en el acceso a un ocio de calidad, a un ocio serio (Stebbins, 2012), a un ocio digno (Cuenca, 2011). El ocio como "generador de sentido" (Cuenca, 2011: 30) se experimenta cuando se ponen en juego la autonomía de la persona, su libertad responsable y la capacidad de implicación desinteresada, aspectos todos ellos educables. Y es este ocio el que deriva en beneficios personales valiosos (desde la perspectiva de la autorrealización, la salud, la ampliación del círculo de relaciones, etc.) y sociales (en cuanto a que el ocio comunitario favorece la integración, la participación, la cohesión social y la identidad). Ello nos obliga a repensar el enfoque tradicional de una formación para el trabajo y contemplar la necesidad de educar para el desarrollo de la persona como miembro activo y participante de la sociedad en todos sus tiempos, de forma que se incremente su bienestar individual y colectivamente (Caballo 2010).

En este sentido, Hutchinson y Robertson (2012) reivindican la necesidad de "alfabetizar en el ocio" para adquirir los conocimientos, habilidades, actitudes y confianza necesaria que permitan conferir un significado personal y positivo a estas experiencias. Pero la educación del ocio no se debe limitar a propiciar el desarrollo de las capacidades individuales, sino que debe visibilizar la necesidad de generar recursos comunitarios que garanticen las condiciones para un ocio valioso que empodere a las personas y a los colectivos; y en esta tarea resulta imprescindible la labor coordinada y compartida entre los poderes públicos, el tercer sector, el ámbito empresarial, las instituciones educativas y los medios de comunicación social.

Esta educación comunitaria del ocio procura modificar las actitudes y hábitos colectivos para hacer efectivo el derecho al ocio, contribuyendo a los 
procesos de desarrollo local, lo que supone activar acciones que ayuden a suprimir las barreras que dificultan el acceso al mismo. Hace ya tiempo que se ha generado consenso en torno a que estas barreras no son sólo de carácter económico, sino también sociales y educativas (falta de hábito, baja percepción de competencia, escasas posibilidades en el entorno, etc.), estando presentes en el ocio juvenil; destacando aquellas relacionadas con la percepción de falta de tiempo, que impiden el disfrute de experiencias de ocio que requieren de perseverancia y constancia, presentándose una brecha de género clara en relación a este aspecto (Videnovi, Peši y Plut, 2010; Madariaga y Romero, 2016).

\section{MÉTODO}

Este artículo toma como referencia el proyecto de investigación De los tiempos educativos a los tiempos sociales: la construcción cotidiana de la condición juvenil en una sociedad de redes. Problemáticas y alternativas pedagógico-sociales. Bajo el acrónimo de RESORTES (EDU2012-39080-C07), fue financiado por el Ministerio de Economía y Competitividad con Fondos FEDER, en el marco del Plan Nacional de I+D+i (2012-2015). Se trata de una investigación en red conformada por seis subproyectos adscritos a otros tantos grupos de investigación pertenecientes a las Universidades de Barcelona, Burgos, Deusto, La Rioja, Santiago de Compostela (coordinadora) y UNED, sustentándose en un enfoque multidisciplinar que parte de los principios del ocio humanista y del reconocimiento de la contribución del ocio al desarrollo.

El objetivo general de este proyecto coordinado se centra en estudiar la naturaleza y alcance de los tiempos educativos y sociales en la construcción cotidiana de la condición juvenil, analizando de qué forma y en qué grado inciden en la vida de los jóvenes pertenecientes a distintos contextos y realidades, identificando sus problemáticas específicas y las alternativas que deberán adoptarse para contribuir al desarrollo integral de su personalidad y al pleno ejercicio de sus derechos cívicos.

De los numerosos objetivos específicos que contempla el estudio, se han seleccionado dos para la elaboración de este artículo:

- Analizar las necesidades y expectativas de la juventud en relación a sus tiempos libres.

- Estudiar las iniciativas de ocio en las que participa la juventud y su congruencia con un ocio positivo.

Se ha trabajado con tres hipótesis: la primera, que las actividades de ocio desarrolladas por los jóvenes -especialmente por los vínculos sociales que generan- resultan esenciales para la construcción de sus identidades juveniles; la 
segunda, que hay un desequilibrio en las dimensiones de ocio a las que se pueden adscribir las actividades que llevan a cabo; por último, que las barreras de tiempo, educativas y económicas están limitando las oportunidades con las que cuentan los chicos y chicas para participar en actividades de ocio valioso.

Los datos presentados se extraen de la segunda aplicación de un cuestionario a alumnado de educación postobligatoria en España (Bachillerato, FP de grado medio y PCPI -actualmente FP básica-). Se ha optado por un muestreo probabilístico estratificado mediante una afijación proporcional en cada una de las seis áreas en las que se dividió el territorio español, tomando como base una simplificación de las diez áreas Nielsen delimitadas por el instituto de investigación de mercados del mismo nombre y asumidas, también, como referencia para numerosos estudios de hábitos poblacionales de diferente naturaleza. Esta opción metodológica ha facilitado un número manejable de estratos con un comportamiento razonablemente homogéneo en el interior y heterogéneo con respecto a las demás zonas.

Son las siguientes:

Zona 1. Noreste: Cataluña, Aragón (excepto Teruel) y Baleares

Zona 2. Levante: Comunidad Valenciana, Murcia y Albacete

Zona 3. Sur: Andalucía, Badajoz, Islas Canarias, Ceuta y Melilla

Zona 4. Centro: Comunidad de Madrid, Castilla La Mancha (excepto Albacete), Castilla y León (excepto León, Palencia y Burgos), Cáceres y Teruel

Zona 5. Noroeste: Galicia, Asturias y León

Zona 6. Norte: Cantabria, País Vasco, La Rioja, Navarra, Burgos y Palencia La aplicación se ha realizado en el curso académico 2015-2016 teniendo en cuenta criterios de ruralidad y urbanidad (mínimo de un centro rural en cada zona, en función de sus características demográficas), también la titularidad del centro (de cada cuatro centros, tres fueron públicos y uno privado).

\section{Ficha técnica de la muestra}

\begin{tabular}{l|l}
\hline Universo & $\begin{array}{l}\text { Alumnado de educación post obligatoria no universitaria } \\
\text { (España) }\end{array}$ \\
\hline Muestreo & $\begin{array}{l}\text { Probabilístico estratificado, mediante afijación proporcional } \\
\text { según el alumnado de cada uno de los estratos }\end{array}$ \\
\hline Muestra & 2.694 cuestionarios válidos recogidos \\
\hline Error absoluto & $1,9 \%$ \\
\hline Nivel de confianza & $95 \%$ dado el supuesto de $\mathrm{p}=\mathrm{q}=0,5$. \\
\hline
\end{tabular}


El cuestionario dirigido al alumnado de Educación Secundaria Postobligatoria se elaboró ad hoc por el equipo interdisciplinar e interuniversitario de investigación, a fin de conocer la percepción de los jóvenes en torno a la vida familiar, el centro escolar y sus tiempos libres. Fue sometido a juicio de catorce expertos externos para garantizar su validez de contenido, realizándose el ajuste final del instrumento a la población sujeto de estudio a través de una aplicación piloto a 140 jóvenes distribuidos en ocho comunidades autónomas. La aplicación fue realizada -previo contacto telefónico con la dirección de los centros escolares- por miembros del equipo investigador en las aulas, durante el transcurso de la jornada lectiva.

Los bloques temáticos en los que se estructura el cuestionario son: datos personales, vida en el centro escolar, vida familiar, tiempo libre, salud y calidad de vida, estudios y mercado laboral en el futuro y emprendimiento. Para la elaboración de este artículo se han utilizado los siguientes ítems del bloque temático "tiempo libre":

- Actividades de ocio (pregunta 21): informa de las tres actividades de ocio que los jóvenes consideran más importantes de entre todas las que practican, clasificadas por orden de relevancia.

- Lugares de realización (pregunta 26): pregunta de respuesta múltiple que da cuenta de los lugares donde suelen practicar las actividades de ocio que consideran más importantes (locales privados, asociaciones, espacios municipales, espacios públicos, naturaleza, etc.)

- Barreras de ocio (pregunta 31 y 32): informa de si los jóvenes tienen que renunciar a alguna actividad de ocio que les gusta y de los motivos que les llevan a declinar dicha práctica (falta de tiempo, falta de dinero, no tener con quién realizarla, falta de decisión, pereza, etc.)

- Nivel de estudios de los padres (pregunta 9): se trata de un ítem procedente del bloque temático "datos personales" que posibilita conocer el nivel de estudios (primarios, secundarios, superiores, sin estudios) de los progenitores.

- Percepción del tiempo durante los días de clase (pregunta 13): informa de su visión acerca de la vivencia del tiempo durante los días lectivos, dentro del espacio escolar y en otros contextos de su vida cotidiana.

En el marco de la investigación, también se aplicaron otros instrumentos (cuestionarios al profesorado y a las familias, entrevistas, grupos de discusión, análisis de dietarios, semanarios y/o agendas personales, buenas prácticas...) que se integran asumiendo una orientación metodológica plural, desde la que se reclama la adaptación concreta de cada estrategia de investigación a los sujetos y realidades objeto de estudio. 


\section{RESULTADOS Y DISCUSIÓN}

Las vivencias cotidianas de las experiencias de ocio por parte de los jóvenes españoles van más allá de la mera ocupación de sus tiempos de libre disposición con actividades de diverso carácter, constituyendo valiosas oportunidades de desarrollo personal y de participación en experiencias enriquecedoras socialmente compartidas. A pesar de que el tiempo libre puede ser negativamente ocupado, muchas de las actividades de ocio practicadas por los jóvenes de 16 a 18 años que cursan Educación Secundaria Postobligatoria conforman convivencias cotidianas - presenciales y virtuales- asociadas a valores, necesidades, prácticas, etc., que resultan esenciales para su desarrollo. Así, el ocio en su doble dimensión personal y social está ligado a la construcción de la identidad juvenil dado que, mediante la participación en diversas actividades, los chicos y chicas experimentan nuevos roles y estilos de vida e interactúan con otros individuos fuera del núcleo familiar, lo que les permite identificarse a sí mismos en relación con los demás (Hendry et al, 2005).

Tabla 1. Actividades de ocio que los jóvenes sitúan entre las tres más importantes de todas las que practican

\begin{tabular}{l|c}
\hline Actividades & $\%$ \\
\hline Deporte & 55,4 \\
\hline Actividades culturales & 54,5 \\
\hline Fiestas & 41,3 \\
\hline Ocio digital & 39,7 \\
\hline Ver la TV & 22,0 \\
\hline Estar con la familia, pareja o amistades & 15,4 \\
\hline Turismo & 8,9 \\
\hline Juegos de mesa y otros & 3,1 \\
\hline Voluntariado y asociacionismo & 1,5 \\
\hline Otras actividades & 17,5 \\
\hline
\end{tabular}

Cuando se les pregunta a los jóvenes españoles por las actividades de ocio que consideran relevantes en su vida, destaca la actividad física -ya sea individual, de competición o al aire libre-al ocupar un lugar preferente en sus tiempos libres, pues un 54,5\% de los jóvenes encuestados la sitúan entre las tres actividades más importantes de todas las que practican. Destaca la preponderancia de las prácticas deportivas frente a otras formas de ocupación de sus tiempos, 
aun siendo muchos los jóvenes que afirman no poder realizar todas las actividades físicas de ocio que les gustaría (por ejemplo, andar en bicicleta, esquiar, hacer escalada, practicar artes marciales, etc.); en este sentido, que para un 20,8\% no haya tiempo suficiente para practicar deporte durante los días de clase, y que para el 56\% se cuente con 'poco' o 'algo' de tiempo para esta actividad, pone de relieve el lugar central que ocupa el tiempo escolar en la organización de la vida cotidiana del alumnado de esta etapa educativa.

La práctica de actividad física por adolescentes y jóvenes ha sido estudiada en múltiples investigaciones (Cano et al., 2011; Fraguela, Varela y Sanz, 2016; Valdemoros, Ponce de León y Gradaílle, 2016) que evidencian la existencia de diferencias en los hábitos deportivos en función del género, así como la disminución de la práctica en las chicas de Educación Secundaria. En el presente estudio se constatan de nuevo dichas diferencias $\left(x^{2}{ }_{11}=477,139 ; \mathrm{p}=0,000\right)$, dado que mientras que para un $66,6 \%$ de los varones el deporte es la actividad considerada más importante entre todas las que realizan, ésta lo es solamente para un 43,6\% de las mujeres (tabla 2). La Women's Sport and Fitness Foundation (s.f.) afirma que a los 16 años se produce un decrecimiento significativo en la participación de las chicas, coincidiendo con el fin de la escolarización obligatoria. Complementariamente, diversos estudios sitúan el problema de la falta de actividad física de las jóvenes como una cuestión preocupante (Macarro, Romero y Torres, 2010; Beltrán et al, 2017), que se manifiesta claramente durante la etapa de Educación Secundaria pero que debe ser abordada desde niveles inferiores, a fin de prevenir las posibles barreras (educativas, sociales, etc.) que limitan sus posibilidades de tener una práctica deportiva satisfactoria y mantener un estilo de vida activo. Wetton et al (2013), en una investigación realizada con chicas adolescentes de 15 y 16 años para evaluar los factores que impiden su participación en deportes de equipo en horario extraescolar, identificaron cuatro barreras destacadas: factores internos, estereotipos, existencia de otros hobbies y actitudes negativas del profesorado.

Para las chicas de entre 16 y 18 años las actividades culturales (ir al teatro, museos, realizar alguna actividad artística, etc.) ocupan el primer lugar por orden de importancia entre sus tres actividades principales de ocio $(64,8 \%)$, frente al $44,9 \%$ de los varones que le otorgan esta consideración. Dentro de este ámbito, entre las actividades que les gustaría realizar con mayor frecuencia -y no realizan- destacan ir al cine y leer. La renuncia a estas actividades puede vincularse a dos razones fundamentales (tabla 3): la falta de tiempo para hacer las cosas que les gustan y la incidencia de motivos de carácter económico, puesto que -en el caso del cine- la escasez de recursos limita las posibilidades de poder asistir a espectáculos culturales; una cuestión que no afecta 
Tabla 2. Actividades de ocio que los jóvenes sitúan entre las tres más importantes, según género

\begin{tabular}{l|c|c}
\hline Actividades & $\begin{array}{c}\text { Chicas } \\
(\%)\end{array}$ & $\begin{array}{c}\text { Chicos } \\
(\%)\end{array}$ \\
\hline Deporte & 43,6 & 66,6 \\
\hline Actividades culturales & 64,8 & 44,9 \\
\hline Fiestas & 45,7 & 37,4 \\
\hline Ocio digital & 31,6 & 47,3 \\
\hline Ver la TV & 21,8 & 22,4 \\
\hline Estar con la familia, pareja o amistades & 16,4 & 14,3 \\
\hline Turismo & 11 & 7,0 \\
\hline Juegos de mesa y otros & 1,6 & 4,5 \\
\hline Voluntariado y asociacionismo & 1,7 & 1,2 \\
\hline Otras actividades & 21,9 & 13,4 \\
\hline
\end{tabular}

tanto a la lectura dado que el acceso a través de bibliotecas o a ejemplares disponibles en el hogar resulta más económico. No obstante, a pesar de estos datos, según la Encuesta de hábitos y prácticas culturales en España (2014-2015) los jóvenes de entre 15 y 19 años constituyen el colectivo que asiste al cine con mayor frecuencia con tasas del $86,3 \%$ en el periodo anual comprendido entre marzo de 2014 y febrero de 2015, por lo que-aunque la crisis financiera haya tenido incidencia en los presupuestos familiares- el cine sigue siendo central en el ocio juvenil.

Otras de las actividades destacadas entre las tres más importantes para los jóvenes son las fiestas y celebraciones (ir de marcha o a discotecas, salir a tomar algo, ir a fiestas tradicionales del barrio/ciudad, etc.), siendo el 41,3\% quienes otorgan a este tipo de prácticas de ocio una posición central. Desde hace décadas, el ocio nocturno y de fines de semana forma parte de las señas de identidad de la juventud española, convirtiéndose las salidas nocturnas en tiempo de diversión para los chicos y chicas y en fuente de preocupación de los adultos, especialmente cuando las experiencias de ocio adquieren una direccionalidad negativa, resultando perjudiciales tanto para los jóvenes como para el entorno en el que se desarrollan. Siguiendo a Gradaílle, Varela y De Valenzuela (2016), la progresiva homogeneización de las actividades lúdicas, el lugar y el tiempo en el que las realizan, comporta nuevos significados para las prácticas de ocio nocturnas; a veces asociadas a experiencias nocivas, como es el 
consumo de drogas y alcohol, que preocupan notablemente a las familias. Sin embargo, mientras los padres y las madres destacan las consecuencias sociales negativas del consumo juvenil, los jóvenes lo banalizan y no lo asocian con problemas personales, de salud y/o de rendimiento (Giménez, Cortés y Espejo, 2010); centrándose en los aspectos positivos que comportan los espacios de socialización con su grupo de iguales.

Mención aparte merece el tiempo dedicado por los jóvenes españoles a las pantallas, referido tanto al ocio digital $(39,7 \%)$ como al consumo de televisión (22\%). Los jóvenes tienen predilección por la realización de actividades con las TIC (navegar por internet, chatear, videojuegos, etc.), presentando el ocio digital una mayor importancia para los varones $(47,3 \%)$ que para las mujeres $(31,6 \%)$. Si bien la exposición a pantallas es elevada para los jóvenes, se detectan diferencias en función del nivel de estudios de sus progenitores -madre $\left(x^{2}{ }_{33}=64,751 ; \mathrm{p}=0,001\right)$ y padre $\left(x^{2}{ }_{33}=61,416 ; \mathrm{p}=0,00\right)$-. Cabe afirmar que cuanto mayor es el nivel de estudios de los padres menor es el tiempo dedicado por los hijos a este tipo de prácticas, especialmente a ver la televisión, pues mientras que el $17 \%$ de hijos con padres con estudios superiores universitarios señalan entre sus tres principales actividades de ocio el consumo de televisión, los chicos y chicas cuyos padres no tienen estudios lo hacen en un 23,5\%. Una radiografía que se repite atendiendo al nivel formativo de la madre, dado que en un 19,7\% de los jóvenes cuyas madres cuentan con estudios superiores universitarios incluyen a la TV entre sus tres principales actividades de ocio, incrementándose esta tasa al 25,8\% cuando son mujeres sin estudios.

En este sentido, es probable que los entornos familiares de nivel educativo alto sean contextos estimulantes de un ocio creativo, antesala del ocio cultural (Ferreira, Pose y De Valenzuela, 2015; López-Sintas, Ghahraman y Pérez Rubiales, 2017); se trata de ambientes favorecedores del ocio como experiencia de desarrollo humano en los que las personas se implican de forma activa. Además, como indican Valdemoros, Sanz y Ponce de León (2017), la cohesión familiar es más saludable cuando los jóvenes no señalan actividades digitales entre sus prácticas predominantes de ocio que cuando indican una o dos prácticas de ocio digital entre sus favoritas; más en concreto, dicho funcionamiento es más sano entre quienes llevan a cabo una sola actividad de ocio digital frente a los que efectúan dos, poniéndose de manifiesto que un menor consumo digital en los hijos se vincula a familias que gozan de una mayor fortaleza en la vinculación emocional entre sus miembros, en la reciprocidad emocional que se da o puede darse entre los componentes de la familia, en la implicación familiar y en el respeto mutuo entre padres e hijos. Siguiendo a Vasco y Pérez Serrano (2017), puede afirmarse que en aquellos ambientes más 
desfavorecidos la población juvenil convierte su inactividad en dilatados espacios de tiempo implementando un ocio habitualmente forzado, sostenido en alternativas escasamente enriquecedoras y en modelos educativos negativos.

En el conjunto de las prácticas de ocio de la juventud resulta preocupante la escasa participación de los chicos y chicas en actividades de voluntariado y asociacionismo pues solo un 1,5\% de los jóvenes encuestados sitúa estas prácticas entre las tres actividades de ocio más importantes para él o ella. Como afirman Gómez-Granell y Julià (2015), los jóvenes están vinculados con el mundo asociativo o cultural o los centros recreativos o de jóvenes principalmente durante la preadolescencia, dado que cuando se hacen mayores dejan de participar progresivamente en los mismos, haciéndose necesario indagar en las razones que contribuyen a que estas opciones les resulten menos atractivas.

Asimismo, actividades como el turismo y los juegos de mesa no suponen para los adolescentes una experiencia de ocio relevante pues, coincidiendo con los datos del estudio de Ricoy y Fernández-Rodríguez (2016), se obtienen tasas de $8,9 \%$ y $3,1 \%$ respectivamente para las actividades mencionadas.

Respecto a los lugares en los que los jóvenes realizan sus prácticas de ocio, destaca la presencia de espacios privados, dado que el domicilio familiar constituye un lugar fundamental para el disfrute del tiempo libre. Resultados previsibles para algunas prácticas como ver la televisión y escuchar la radio (el $76,6 \%$ de las veces se lleva a cabo en casa) o el ocio digital (52,1\% de las ocasiones que se practica se realiza en casa), pero que sorprenden en relación a las actividades culturales o los hobbies (escuchar música, ver películas, hacer alguna actividad artística, etc.), que son desarrolladas el $44,4 \%$ de las veces en residencias privadas. La práctica de actividad física de ocio se realiza fundamentalmente en clubes deportivos (el 20,1\% de las veces) y en polideportivos (el 27,7\% de las ocasiones), mientras que los centros comerciales tienen una presencia notable en las fiestas o celebraciones (30,8\% de las veces que participan en este tipo de actividades lo hacen en estos centros) y en el tiempo compartido con la familia, pareja o amistades (23,3\% de las ocasiones). También la calle tiene una presencia destacada para el desarrollo de las dos últimas actividades mencionadas -fiestas o celebraciones y estar con la familia, pareja o amistades- puesto que los jóvenes indican que el $26 \%$ de las veces que participan en fiestas lo hacen en la calle y en el 26,8\% de las ocasiones están con sus familiares o amigos en el espacio urbano. En relación al tiempo que pasan con la familia, pareja o amistades también señalan el hogar como espacio preferente ( $21,4 \%$ de las veces) lo que lleva a pensar que, en el conjunto de los espacios de ocio, los centros comerciales y la calle son lugares de socialización habi- 
tuales de los chicos y chicas con su grupo de iguales mientras que es en el domicilio donde pasan la mayor parte del tiempo con sus padres. Asimismo, cabe mencionar que el centro escolar no es percibido por los jóvenes como un espacio de ocio, no superando el $7 \%$ las veces en que realizan en la escuela este tipo de prácticas, independientemente de su tipología (deportiva, lúdicofestiva, etc.).

En este marco, resulta necesario poner de manifiesto la existencia de barreras de ocio en la etapa juvenil, un conjunto de factores que no son solo de carácter económico, sino también social y educativo. Así se reconoce en la Carta Internacional para la Educación del Ocio (WLRA, 1994) cuando se afirma que los factores políticos, económicos, sociales, culturales y medioambientales pueden aumentar o dificultar el ocio. En el estudio realizado, se detecta que son múltiples las razones que obligan a los jóvenes a renunciar a actividades que les resultan de interés (tabla 3 ), destacando fundamentalmente la falta de tiempo $(40,1 \%)$, la falta de dinero $(9,6 \%)$ y el exceso de tareas escolares $(8,6 \%)$.

Tabla 3. Motivos de renuncia de los jóvenes a actividades de ocio que les gustan

\begin{tabular}{l|c}
\hline Motivo & $\%$ \\
\hline Falta de tiempo & 40,1 \\
\hline Falta de dinero & 9,6 \\
\hline Porque tiene muchas tareas escolares & 8,6 \\
\hline Horarios incompatibles & 4,9 \\
\hline Falta de decisión, pereza & 3,1 \\
\hline No hay donde hacerlo en su localidad & 2,3 \\
\hline No le deja su familia & 2,0 \\
\hline Dificultades de transporte & 1,4 \\
\hline No tiene con quién & 1,1 \\
\hline Otras causas & 26,9 \\
\hline
\end{tabular}

En el conjunto de los tiempos cotidianos, el tiempo escolar ocupa para los adolescentes y jóvenes de entre 16-18 años un papel central en los días lectivos, siendo el eje articulador de casi todas las actividades que realizan. Aunque el grado de fatiga que manifiestan al acabar las clases no es elevado -se concentran en torno $52 \%$ las respuestas referidas a las categorías 'poco-algo'-, solamente un $11,5 \%$ indica que el tiempo que están en el centro les resulta 'bastante' o 
'muy' corto, señalando un 36,3\% que les parece largo. Esta visión contrasta con el tiempo que pasan fuera del centro escolar, siendo escaso para el 69,1\% de los jóvenes encuestados. Una situación que evidencia el deseo de los chicos y chicas de contar con mayor disponibilidad horaria para la realización de otras actividades, pues el 56,3\% afirma que el tiempo del que disponen para hacer aquello que les gusta es escaso ('poco'-'algo').

Tabla 4. La percepción del tiempo en los días de clase

\begin{tabular}{l|c|c|c}
\hline \multirow{2}{*}{ Los días de clase... } & \multicolumn{3}{|c}{ Porcentaje (\%) } \\
\cline { 2 - 4 } & Nada & Poco-Algo & Bastante-Mucho \\
\hline $\begin{array}{l}\text { Me siento agobiada/o por todo lo que } \\
\text { tengo que hacer }\end{array}$ & 9,2 & 46,6 & 44,2 \\
\hline Me siento fatigada/o al acabar las clases & 17,7 & 52,2 & 30,1 \\
\hline $\begin{array}{l}\text { El tiempo que paso en el centro se } \\
\text { me hace corto }\end{array}$ & 36,3 & 52,1 & 36,1 \\
\hline $\begin{array}{l}\text { El tiempo que paso fuera del centro se } \\
\text { me hace corto }\end{array}$ & 6,8 & 24 & 69,1 \\
\hline
\end{tabular}

(Estimación a partir de los datos porcentuales agrupados en tres grupos: nada; pocoalgo; bastante-mucho; $\mathrm{n}=2694$ )

Estos datos desvelan que la falta de tiempo durante los días lectivos para la realización de actividades que les gustan y que no sean las estrictamente académicas es evidente, siendo la opción 'poco-algo' predominante en todos los ítems analizados (tabla 5). De lunes a viernes, la sensación de falta de tiempo se acentúa en lo referido a estar con los amigos, estar con la familia o practicar algún deporte, aproximándose al 50\% quienes afirman tener 'poco' o 'algo' de tiempo para este tipo de acciones. Resulta relevante que un $37,1 \%$ manifieste no disponer de 'nada' de tiempo para asistir a actividades extraescolares; una cuestión que en la etapa postobligatoria puede vincularse al incremento del tiempo de estudio y a la presencia de aficiones ya consolidadas, realizándose una selección más deliberada de las actividades de ocio que se practican.

Del conjunto de ítems analizados, los porcentajes de respuesta más elevados -en las categorías 'bastante-mucho'- se concentran en el tiempo que pasan con la familia, que consideran suficiente.

Aunque la mayoría de los jóvenes encuestados indican que no renuncian a ninguna actividad que les gusta (62,3\%), un $37,7 \%$ afirma tener que prescindir de la realización de determinadas prácticas, existiendo diferencias en función 
Tabla 5. Tiempo disponible para la realización de actividades no académicas durante los días de clase

\begin{tabular}{l|c|c|c}
\hline \multirow{2}{*}{ Tengo tiempo suficiente para... } & \multicolumn{3}{|c}{ Porcentaje (\%) } \\
\cline { 2 - 4 } & Nada & Poco-Algo & Bastante-Mucho \\
\hline Estar con mis amigas o amigos & 8,3 & 56 & 35,7 \\
\hline Estar con mi familia & 4,4 & 48,8 & 46,9 \\
\hline Practicar deporte & 20,8 & 48 & 31,2 \\
\hline Ir a actividades extraescolares & 37,1 & 42 & 20,8 \\
\hline Hacer las cosas que me gustan & 7,7 & 56,3 & 36,1 \\
\hline
\end{tabular}

(Estimación a partir de los datos porcentuales agrupados en tres grupos: nada; pocoalgo; bastante-mucho; $\mathrm{n}=2649$ )

del género $\left(x^{2}{ }_{1}=54,845 ; \mathrm{p}=0,000\right)$; son las mujeres (45\%) quienes renuncian en mayor medida que los varones $(30,8 \%)$ a hacer alguna actividad que desearían realizar en su tiempo de ocio. La disponibilidad de más tiempo para el ocio por parte de los hombres ha sido puesto de manifiesto en el Informe Juventud en España (2012), según el cual los hombres de 15 a 29 años afirman tener una hora libre más a la semana para su ocio (33,10 horas semanales) que las mujeres de la misma edad (32,10 horas semanales), estando las actividades a las que dedican el tiempo bastante diferenciadas por género. Complementariamente, diversos estudios (Samdhal, 2013; Megías y Ballesteros, 2014) aluden a la existencia de barreras y desigualdades entre chicos y chicas, tanto en la estructura como en la cantidad de ocio disponible, así como respecto al sesgo en las libertades que los progenitores conceden a sus hijos en función del género, afirmando que existe un mayor control familiar en cuanto a restricciones horarias, compañías y lugares de destino en el caso de las mujeres, que se incrementa en los inicios de la adolescencia.

\section{CONCLUSIONES}

La socialización en experiencias de ocio valioso genera diferencias en la construcción de las identidades juveniles, puesto que el conjunto de saberes, habilidades e intereses que se pueden desarrollar en contextos desfavorecidos difiere ampliamente de las oportunidades con las que se cuenta en escenarios educativamente enriquecedores. Una cuestión a la que se añaden los roles diferenciados que chicos y chicas asumen en esta etapa del ciclo vital y la persistencia de barreras que limitan las posibilidades de las mujeres para participar en actividades de ocio que desearían realizar. En líneas generales, la falta 
de tiempo constituye una de las principales barreras que limitan la participación de las chicas y chicos en actividades de ocio que les gustan, destacando su deseo de contar con más tiempo durante los días lectivos para poder implicarse en iniciativas que no sean estrictamente académicas. Junto a la presión temporal y las limitaciones económicas, la falta de contextos estimulantes de un ocio creativo restringe la participación en experiencias de ocio que requieren aprendizaje y formación; de ahí la necesidad de educar en un ocio que amplíe los horizontes (Caride, 2012), no solo en las primeras edades sino a lo largo de toda la vida.

Los resultados obtenidos ponen de manifiesto que las vivencias de los tiempos libres de los jóvenes que cursan Educación Secundaria Postobligatoria se desarrollan fundamentalmente a través de prácticas de ocio vinculadas a las dimensiones lúdica (deporte, ocio digital), creativa (actividades culturales) y festiva, siendo residual su participación en iniciativas de carácter solidario (Caballo, 2015). En concreto, cabe destacar la escasa implicación cívica de los jóvenes en tareas de voluntariado o mediante el asociacionismo. Una cuestión que resulta preocupante si se considera que las experiencias vitales asociadas a la dimensión solidaria del ocio son fundamentales para la construcción de la identidad juvenil al reportar importantes beneficios personales y sociales, en un tiempo que siendo compartido de forma voluntaria y desinteresada se orienta al ejercicio de la ciudadanía activa. No obstante, internet y los medios sociales están cambiando los modos de ejercer la dimensión solidaria dando lugar al voluntariado virtual (Herrán y Viñals, 2011) y a nuevas formas de participación ciudadana en modo online que modifican las formas que las personas tienen de relacionarse e interactuar (Subirats, 2011), generando vínculos y lazos horizontales que suponen un cambio profundo en la concepción del ocio solidario que ha de incorporar las potencialidades del nuevo escenario que genera internet.

\section{BIBLIOGRAFÍA}

Beltrán, V.J. et al. (2017). Diferencias según género en el tiempo empleado por adolescentes en actividad sedentaria y actividad física en diferentes segmentos horarios del día. Retos, 31, 3-7.

Caballo, M.B. (2010). Educación do lecer. En J.A. Caride y F. Trillo (dir), Diccionario Galego de Pedagoxía. Vigo: Xerais, 226-228.

Caballo, M.B. (2015). Jóvenes, ocio y educación en la sociedad red. Pedagogía Social. Revista Interuniversitaria, 25, 17-24.

Cano, A.C., Pérez, I., Casares, I. y Alberola, S. (2011). Determinantes del nivel de actividad física en escolares y adolescentes: estudio OPAC. Anales de Pediatría, 74(1), $15-24$. 
Caride, J.A. (2012). Lo que el tiempo educa: el ocio como construcción pedagógica y social. Arbor, 754, 301-313.

Cobo, C. y Moravec, J. W. (2011). Aprendizaje invisible: Hacia una nueva ecología de la educación. Barcelona: Laboratori de Mitjans Interactius/Publicacions i Edicions de la Universitat de Barcelona.

Cuenca, M. (2011). Valores que dimanan del ocio humanista. En A. Madariaga y J. Cuenca (eds.), Los valores del ocio: cambio, choque e innovación. Documentos de Estudios de Ocio, 43, 17-48. Bilbao: Universidad de Deusto.

Cuenca, M. (2014). Ocio valioso. Documentos de Estudios de Ocio, 52. Bilbao: Universidad de Deusto.

Etxeberria, B. y Segú, M. (2011). Gaztelekus de Gipúzkoa. Fomentando un ocio lúdico y solidario. En R. Ahedo y F. Bayón (eds.), OcioGune 2011. Ocio e innovación social: hacia un ocio comprometido con el desarrollo humano. Comunicaciones. Cuadernos de estudios del Ocio, 14, 121-129. Bilbao: Universidad de Deusto.

Ferreira, P., Pose, H. y De Valenzuela, A.L. (2015). El ocio cotidiano de los estudiantes de Educación secundaria en España. Pedagogía Social. Revista Interuniversitaria, 25, 25-49.

Fraguela, R., Varela, L. y Sanz, E. (2016). Ocio deportivo, imagen corporal y satisfacción vital en jóvenes españoles. Revista de Psicología del Deporte, 25(2), 33-38.

García Canclini, N. (2014). Innovaciones en los estudios sobre los jóvenes y la lectura. En MINEDUC, Plan Nacional de la Lectura. Actas del II Seminario Internacional ¿Qué leer? ¿Cómo leer?: Lecturas de Juventud. Santiago de Chile: MINEDUC.

Giménez, J.A., Cortés, M.T. y Espejo, B. (2010). Consumo de alcohol juvenil: una visión desde diferentes colectivos. Health and Addictions, 10(1), 13-34.

Gómez-Granell, C. y Julià, A. (2015). Tiempo de crecer, tiempo para crecer. Barcelona: Ajuntament de Barcelona.

González, Y. y Feixa, C. (2013). La construcción histórica de la juventud en América Latina. Santiago: Editorial Cuarto Propio.

Gradaílle, R., Varela, L. y De Valenzuela, A.L. (2016). Preocupaciones del profesorado y de las familias sobre los tiempos escolares y de ocio del alumnado de Educación Secundaria Postobligatoria. Revista interuniversitaria de formación del profesorado, 86, 19-62.

Hendry, L.B., Shucksmith J., Love, J.G. y Glendinning, A. (2005). Young people’s leisure and lifestyles. London: Routledge.

Herrán, A. y Viñals, A. (2011). Internet y los medios sociales como experiencia de ocio digital solidario entre los jóvenes. Un paso hacia un ocio digital más humanista. En R. Ahedo y F. Bayón (eds.), OcioGune 2011. Ocio e innovación social: hacia un ocio comprometido con el desarrollo humano. Comunicaciones. Cuadernos de estudios del Ocio, 14, 247-258. Bilbao: Universidad de Deusto.

Hutchinson, S. y Robertson, B. (2012). Leisure education: a new goal for an old idea whose time has come. Pedagogía Social. Revista interuniversitaria, 19, 127-139.

INJUVE (2012). Informe 2012. Juventud en España. Recuperado de http://www.injuve.es/ 
López-Sintas, J., Ghahraman, A.y Pérez Rubiales, E. (2017). Young people’s leisure patterns: testing social age, social gender, and linguistic capital hypotheses. Journal of Youth Studies, 20(2), 180-199.

Macarro, M., Romero, C. y Torres, J. (2010). Motivos de abandono de la práctica de actividad físico-deportiva en los estudiantes de bachillerato de la provincia de Granada. Revista de Educación, 353, 495-519.

Madariaga, A. y Romero, S. (2016). Barreras percibidas entre los jóvenes para no participar en actividades de ocio. Revista de Psicología del Deporte, 25(2), 21-26.

MECD (2015). Encuesta de hábitos y prácticas culturales en España (2014-2015). Madrid: Ministerio de Educación, Cultura y Deporte.

Megías, I. y Ballesteros, J. (2014). Jóvenes y género. Estado de la cuestión. Madrid: FAD.

Reig, D y Vílchez, L. (2013). Los jóvenes en la era de la hiperconectividad: tendencias, claves y miradas. Madrid: Fundación Telefónica.

Ricoy, M.C. y Fernández Rodríguez, J. (2016). Prácticas y recursos de ocio en la adolescencia. Educatio Siglo XXI, 34(2), 103-124.

Samdahl, D. (2014). Women, gender and leisure constraints. En V. Freysinger, S.M. Shaw, K.A. Henderson y M.D. Bialeschki, Leisure, women and gender (pp. 109-126). Urbana, IL: Venture Publishing.

Stebbins, R.S. (2012). Compromiso temporal discrecional: efectos sobre la elección y el estilo de ocio. Arbor, 188(754), 293-300.

Subirats, J. (2011). Otra sociedad, ¿otra política?. Del «no nos representan» a la democracia de lo común. Barcelona: Icaria.

Valdemoros, M.A., Ponce de León, A. y Gradaílle, R. (2016). Actividad física de ocio juvenil y desarrollo humano. Revista de Psicología del Deporte, 25(2), 45-51.

Valdemoros, M.A., Sanz, E. y Ponce de León, A. (2017). Ocio digital y ambiente familiar en estudiantes de Educación Postobligatoria. Comunicar: Revisa científica iberoamericana de comunicación y educación, 50, 99-108.

Vasco, M. y Pérez Serrano, G. (2017). Ocio digital en los jóvenes en dificultad social. Bordón. Revista de pedagogía, 69(2), 147-160.

Videnovi , M., Peši , J. y Plut, D. (2010). Young people's leisure time: gender differences. Psihologija, 43(2), 199-214.

Wetton, A.R., Radley, R., Jones, A.R. y Pearce, M.S. (2013). What are the barriers which discourage 15-16 years-old girls from participating in team sports and how can we overcome them?. BioMed Research International, 2013, 1-8.

MARÍA BELÉN CABALLO VILLAR: Doctora en Ciencias de la Educación por la Universidad de Santiago de Compostela y profesora titular de Pedagogía Social en esta misma Universidad desde el año 2003, es miembro del grupo de investigación en Pedagogía Social y Educación Ambiental (SEPA-interea). Sus publicaciones y líneas de investigación se centran en las siguientes temáticas: políticas socioeducativas y desarrollo comunitario local, ciudades educadoras, tiempos educativos y tiempos sociales, y pedagogía del ocio. 
Datos de contacto: Departamento de Pedagogía y Didáctica. Facultad de Ciencias de la Educación-Campus vida. Rúa Prof. Vicente Fráiz Andón, s/n. 15782, Santiago de Compostela. belen.caballo@usc.es

LAURA VARELA CRESPO: Doctora en Ciencias de la Educación, Licenciada en Pedagogía y Diplomada en Educación Social, es miembro del grupo de investigación en Pedagogía Social y Educación Ambiental (SEPA-interea). Profesora Ayudante Doctora en el Departamento de Pedagogía y Didáctica de la Universidad de Santiago de Compostela. Sus investigaciones y publicaciones se centran en la educación social, los servicios sociales, los tiempos escolares y la pedagogía del ocio.

Datos de contacto: Departamento de Pedagogía y Didáctica. Facultad de Ciencias de la Educación-Campus vida. Rúa Prof. Vicente Fráiz Andón, s/n. 15782, Santiago de Compostela. laura.varela@usc.es

EUSEBIO MANUEL NÁJERA MARTÍNEZ: Profesor de Filosofía, Magister en Educación de Adultos y Jóvenes. Trabajo profesional de docencia, investigación y asesoría en la educación superior, formación de formadores y profesionales de programas y proyectos educativos. Actualmente es coordinador de Prácticas Profesionales en el Instituto de Filosofía, PUCV. Miembro del Consejo Ejecutivo de la Sociedad Iberoamericana de Pedagogía Social (SIPS).

Datos de contacto: Instituto de Filosofía. Avenida El Bosque 1290, Sausalito, Viña del Mar, Chile. eusebio.najera@pucv.cl

Recibido: 05/04/2017

Aceptado: 16/09/2017 


\title{
SERIOUS AND CASUAL LEISURE ACTIVITIES IN THE CONSTRUCTION OF YOUNG ADULT IDENTITY: A STUDY BASED ON PARTICIPANTS' SELF-DESCRIPTIONS \\ LOS OCIOS SERIO Y CASUAL EN LA CONSTRUCCIÓN DE LA IDENTIDAD JUVENIL. UN ESTUDIO BASADO EN LA AUTOEXPRESIÓN DE LOS PARTICIPANTES
}

\author{
Nuria Codina \\ Department of Social Psychology and Quantitative Psychology \\ University of Barcelona, Spain \\ ncodina@ub.edu \\ Jose V. Pestana \\ Department of Social Psychology and Quantitative Psychology \\ University of Barcelona, Spain \\ jvpestana@ub.edu \\ Robert A. Stebbins \\ Department of Sociology \\ University of Calgary, Canada \\ stebbins@ucalgary.ca
}

Cómo citar / Citation

Codina, Nuria; Pestana, Jose V. y Stebbins, Robert A. (2017) "Serious and Casual Leisure Activities in the Construction of Young Adult Identity: A Study Based on Participants' Self-Descriptions". OBETS. Revista de Ciencias Sociales, 12(Extra 1): pp-pp. 65-80. doi:10.14198/OBETS2017.12.1.12

\section{Abstract}

Leisure activities and identity interact and structure young people's lives. Empirical research on such interaction is scarce and contradictory. This paper explores the relationships between leisure activities with different levels of 
commitment (serious, casual) and identity traits. A total of 938 young people (476 men and 462 women) aged 18 to $24(M=21.13$; DT $=1.98)$ completed a time budget (TB) and a self-description questionnaire about identity based on the Twenty-Statements Test, as well an association between the TB and the self-descriptions. The study shows the complementarity of serious and casual leisure in the formation of youth identities and gender differences.

Keywords: Leusure; Youth Leisure; Identity; Serious Leisure Perspective; Time Budget Technique; Twenty-Statements Test

\section{Resumen}

Los ocios y la identidad interaccionan y vertebran la vida en la juventud. Las investigaciones empíricas sobre dicha interacción son escasas y contradictorias. Este trabajo estudia las relaciones entre ocios con diferentes implicaciones (serio, casual) y rasgos identitarios. 938 jóvenes (476 hombres y 462 mujeres) de entre 18 y 24 años $(M=21,13 ; D T=1,98)$ respondieron: un Presupuesto de Tiempo (PT); un cuestionario de autodescripciones identitarias basadas en el Twenty-Statement Test (TST) y una prueba de asociación entre el PT y las auto-descripciones. Se muestra la complementariedad de ocios serios y casuales en la configuración de las identidades juveniles y diferencias de género.

Palabras claves: Ocio, Ocio juvenil, Identidad, Perspectiva del Ocio Serio, Presupuesto de tiempo, Twenty-Statement Test

\section{INTRODUCTION}

Youth is one of the most relevant stages of human development; it is important and distinct from other phases because of the numerous, rapid, physical, biological and psychosocial changes experienced. Among these changes, the quest for autonomy and freedom and personal relationships with peers have enormous psychosocial content, and leisure -when not the medium- is closely linked to this quest. In effect, the sphere of leisure activities plays a fundamental role, since it is there -more than in other areas of everyday life- that young people have greater opportunities to exercise their autonomy and freedom to be and not be with certain people, as well as doing and not doing certain activities (Codina, 1986; Silbereisen \& Todt, 1994). The greatest possibilities of discovering their interests and developing their personal identity emerge in relation to these two freedoms of being and doing (Erikson, 1968; Kleiber, 1999; Waterman, 1990). In this sense, it is important not to ignore the fact that, between the genders, there are not only differences in levels of participation and interest in certain leisure activities and behaviors, but also in the experience and role of leisure in everyday life and, consequently, in the development of social identity (Codina, Pestana, Castillo, Balaguer, 2016; Henderson \& Shaw, 2006). 
The majority of authors maintain that the identity process is one of the most active in youth -and that leisure activities take the center stage in this process. However, these assertions are not generally accompanied by rigorous empirical studies that demonstrate this relationship in detail (Eccles \& Barber, 1999; Fredricks, Alfeld-Liro, Hruda, Eccles, Patrick, \& Ryan, 2002; Hansen, Larson, \& Dworkin, 2003; Kleiber, Walker \& Mannell, 2011; Larson, 2000; Stebbins, 2001, Verma \& Larson, 2003). In fact, the findings of the studies carried out to date have brought to light important nuances and contradictions. For example, it has been observed that not all leisure activities contribute to the optimal development of the young adult, nor are they experienced equally. In this respect, Eccles and Barber (1999) have shown that structured activities -organized on a regular timetable and displaying commitment, guidance, direction, progressively greater goals, complexity and challenges- correlate with socially desirable behaviors (Mahoney \& Stattin, 2000; Mahoney, Stattin \& Lord, 2004). In contrast, poorly structured activities are related to behaviors valued as socially negative. For their part, Shin and You (2013) have found that active leisure has more positive effects than non-active or social leisure, because individual identity is much more involved in the former. This is due to the fact that active leisure activities offer values and challenges that help the individual to feel part of the activity and reflect on the correspondence between investment and benefits (Burke 1991; Coatsworth Sharp, Palen, Darling, Cumsille, \& Marta, 2005; Shin \& Oh, 2012; Shin \& You, 2013). As can be seen, structured leisure does not satisfy the need for autonomy and independence sought by young people. Nonetheless, there is a leisure mode that does present some of the requirements; we are referring to serious leisure, which consubstantially requires some degree of commitment to the activity.

In effect, some of the parameters evaluated to reveal the different relationships between types of leisure activities and identity are found in serious leisure (Stebbins, 1992); this type of leisure is characterized by the commitment and dedication shown by the participants, who seek to improve their skills in the activity -so that the performance of the activity follows a developmental process similar to that of a professional career, generating its own social world(Codina, 1999; Stebbins, 2006). Identity is one of the core aspects of this serious leisure process. In the words of Stebbins himself (2001: 56) "every serious leisure activity offers a major lifestyle and identity for its enthusiasts".

Although it seems that participation in a serious leisure activity enables the individual to acquire a positive sense of identity (Green \& Jones, 2005), other types of leisure are also essential for its optimal development (Codina \& Pestana, 2008; Kleiber, Walker, \& Mannell, 2011), including -and in relation to 
serious leisure- casual leisure, a type of leisure that does not pursue specific goals. Thus, as argued by the Serious Leisure Perspective (SLP) itself, an optimal leisure lifestyle consists of an appealing balance of serious and casual leisure (Stebbins, 2005). On the other hand, when there is over-identification with an activity (for example, sport), this prevents the person from participating in other leisure experiences and thereby experiencing other roles or identities -and so identity is constructed on a one-dimensional basis- (MacCoshan \& Gravelle, 2016; Miller \& Kerr, 2002; Werthner \& Orlick, 1986). To better understand the potential of these two leisure modes individually and in conjunction, we will first review the concepts.

Serious leisure is a category of the SLP, which in people's everyday lives is defined as "the systematic pursuit of an amateur, hobbyist, or volunteer core activity that is highly substantial, interesting and fulfilling, and where, in the typical case, participants find a career in acquiring and expressing a combination of its special skills, knowledge, and experience" (Stebbins, 1992: 3). Some types of leisure activities tend to become serious because of the way they are practiced, such as physical and sports activities, music, or collecting. The studies that compare those people who do serious leisure and those who do not show that the former stand apart in the following ways: 1) they develop a unique ethos on becoming involved in the social world of the activity performed; 2) they obtain lasting benefits such as self-fulfillment and self-enrichment; 3) they show perseverance; 4) they make a significant personal effort; 5) they manage the leisure activity as if it were a career "in leisure" (equivalent to a professional career); and 6) they strongly identify with the activity (Elkington \& Stebbins, 2014; Stebbins, 2015). All told, these qualitative dimensions describe how the process of serious leisure becomes a central component of people's lives.

On the other hand, casual leisure is "immediately, intrinsically rewarding; and it is a relatively short-lived, pleasurable activity requiring little or no special training to enjoy it. It is fundamentally hedonic; it is engaged in for the significant level of pure enjoyment, or pleasure, found there" (Stebbins, 1997: 18). Activities considered casual leisure include relaxation (taking a nap), active entertainment (meeting up to play), passive entertainment (watching television), sensory stimulation (e.g. drinking alcohol) and social conversation. The main benefits of casual leisure have been listed as the development of creative and discovery skills, restorative power, maintenance of interpersonal relations, and improved wellbeing and quality of life (Elkington \& Stebbins, ibid.). For their part, Hutchinson and Kleiber (2005) point out that casual leisure provides protection against stress, by aiding self-recovery (when expe- 
riencing pleasure in situations of stress), besides favoring processes of change conducive to personal development (for example, by altering priorities or revealing new paths in life). Thus, it is clear that casual leisure complements serious leisure in the sense that it provides an opportunity to take a rest from the intensity of serious leisure (Stebbins, 1997). And also, as noted earlier, it is an important asset for the construction of identity, since it provides variety.

Although the majority of authors do not hesitate to affirm the necessary complementarity of leisure activities in the construction of identity, the empirical findings are partial. On the one hand, in the research done on serious leisure, various specific activities (such as kayaking, skydiving, climbing or running) have been studied to highlight the relationship between serious leisure and identity, using qualitative methods to obtain a conceptual structure of the interrelationship between identity and serious leisure. On the other hand, there are no studies of the relationship between casual and serious leisure (MacCosham \& Gravelle, 2016).

In view of the above, the goal of this research was to learn about young people's leisure activities -both serious and casual- and main identity traits, and the link between identity traits and the aforesaid leisure activities. These relationships allow us to formulate the hypothesis that guides this study, according to which the serious and casual leisure are related in a differential way with respect to the identity traits of the youth.

\section{METHODOLOGY}

\section{Participants}

A total of 938 young people ( 476 boys and 462 girls) aged between 18 and 24 years old ( $M=21.13$ years; $S D=1.98)$ participated in a study on time use among young people in Spain (for further details, see the note on research funding). The sample was obtained from an online panel using proportional allocation, for a $95 \%$ confidence level and a margin of error of $3.2 \%$. The quotas set for the sample -based on the Spanish population census dated 01/01/2015 (INE, 2015)- were sex and age (the aforementioned 18 to 24-year-old age bracket)

\section{Instruments}

Two tests were used. The first one consisted of an ad hoc questionnaire with the structure and characteristics of the Time Budget Technique (TBT), designed along the lines of the instrument proposed by Neulinger (1986) in his research. Specifically, this study used the adaptations and applications introduced for 
various studies carried out in the Spanish context (Codina, 1999, 2007; Codina $\&$ Pestana, 2009, 2016; Codina, Pestana, Balaguer \& Castillo, 2016). The activities carried out on the day before the day the data was collected were registered in the TBT used for this research.

After completing the TBT, in the second test the respondents were asked about their most important character traits (i.e. self-descriptions), a question based on the instrument known as the Twenty-Statement Test (TST: Kuhn \& McPartland, 1954). This has proved to be a sensitive test for capturing a wide variety and wealth of self-descriptions (in our case, see the studies by Codina, 1998; Codina \& Pestana, 2008; Escobar, 1983; Escobar, Montes \& SánchezSierra, 2015; Morales, 1989).

\section{Procedure}

The fieldwork was preceded by two preparatory phases that followed the guidelines of previous research developed in the field of leisure activities (Codina $\&$ Pestana, 2012, 2016). In the first phase, the research team worked with specialized technical staff to introduce the items into the software in the format the participants would see. To prevent data loss, the questionnaire was programmed so that, to complete it, each of the questions had to be answered in order. In other words, progress could only be made when the previous question on the screen had been answered (otherwise, the users were reminded by a pop-up window). The response categories for each question appeared on the same screen so as to avoid the need to scroll down. Once the final programming of the questionnaire had been verified, a pilot test was carried out. The results of this test were used to make any necessary adjustments to the format.

After a last performance check, the participants were sent an e-mail inviting them to take part in the study, with a direct link to the instrument (a single-use link, which could not be re-opened once the answers had been sent). Access to the questions was set for November 2015. The invitation was only extended to persons who met the established age requirement.

\section{Data analysis}

When processing the findings, the following variables were considered: characteristics of the sample (gender and age), leisure activities done and self-descriptions. In order to classify the responses obtained about leisure activities, we followed the guidelines approved by the European Union for validating research on time use (EUROSTAT, 2009). As regards the categorization of self-descriptions, we followed the criteria established by Escobar et al. (2015). These 
authors have drawn on the analysis carried out by Kuhn and McPartland (1954) on the subjective meaning of the definitions that people provide about themselves (also called sub-consensual statements) to define five attitudinal categories: 1) self-evaluations, through which individuals express their way of being in the light of six possible dimensions -intellectual aptitudes (competencies that are not directly observable), practical aptitudes (observable behavioral competencies), character and morals (self-reflections), social life (relational characteristics), appearance (evaluation of physical self-image) and emotional outlook (state of mind); 2) self-esteem, where people express their degree of satisfaction with themselves; 3 ) preferences, description of personal tastes; 4) beliefs, expression of opinions about reality; and 5) ambitions, statements regarding their own future.

As appropriate, the associations between the variables were calculated using the Chi square coefficient (between participants' gender, leisure activities and self-descriptions). For reasons of brevity, only the cases with the most significant associations are shown in the tables. Confidence levels considered in data analysis correspond basically to those recommended by the American Psychological Association ("a 95\% or 99\% confidence interval": APA, 2010, p. 34); even though, if an association with a confidence level around $90 \%$ brings into light an issue of importance related to the research carried out, this association is also underlined.

\section{RESULTS AND DISCUSSION}

\section{Young adults' leisure activities and their relationship with identity}

Taking the sample as a whole, the leisure activity (Table 1 ) practiced by over half the participants was watching television, DVD or videos $(n=518,55.2 \%)$. This was followed by social life $(n=518,55.2 \%)$ and listening to the radio or recordings $(n=216,23.0 \%)$. Physical exercise $(n=147,15.7 \%)$ and computer use ( $n=139,14.8 \%)$ displayed percentages of around $15 \%$. When analyzing leisure activities according to gender, significant differences were observed in social life $\left(\chi^{2}=4.16, p=.041\right)$, physical exercise $\left(\chi^{2}=2.85, p=.091\right)$ and computer use $\left(\chi^{2}=3.82, p=.082\right)$. As far as social life is concerned, girls were more active (53.6\%, compared to $46.9 \%$ of men). On the other hand, men scored higher on physical exercise and computer use (respectively, $57.1 \%$ and $57.6 \%$, compared to $42.9 \%$ and $42.4 \%$ among girls).

When defining themselves (Table 2), most participants provided selfdescriptions relating to both character and moral traits $(n=739,78.8 \%)$, as well as about how they relate socially $(n=638,68.0 \%)$. In contrast to this trend, it can be seen that lesser numbers provided self-descriptions about their 
Table 1. Leisure activities practiced. Prevalence according to gender

\begin{tabular}{l|r|r|r|r|r|r}
\hline & \multicolumn{2}{|c|}{$\begin{array}{c}\text { Total sample } \\
(N=938)\end{array}$} & \multicolumn{2}{c|}{ Gender } & & \\
\hline Leisure activities & \multicolumn{1}{|c|}{$n$} & \multicolumn{1}{c|}{$\%$} & $\begin{array}{r}\text { Men } \\
(n=476)\end{array}$ & $\begin{array}{r}\text { Woman } \\
(n=462)\end{array}$ & \multicolumn{1}{c}{$\chi^{2}$} & \multicolumn{1}{c}{$p$} \\
\hline Social life & 347 & 37.0 & 46.4 & 53.6 & 4.16 & .041 \\
Entertainment and & 32 & 3.4 & 56.3 & 43.8 & .40 & .526 \\
culture & & & & & & \\
Physical exercise & 147 & 15.7 & 57.1 & 42.9 & 2.85 & .091 \\
Arts and hobbies & 26 & 2.8 & 50.0 & 50.0 & .00 & .938 \\
Computer use & 139 & 14.8 & 57.6 & 42.4 & 3.02 & .082 \\
Reading & 202 & 21.5 & 47.5 & 52.2 & 1.06 & .301 \\
Watching TV, DVDs & 518 & 55.2 & 50.4 & 49.6 & .06 & .806 \\
or videos & & & & & & \\
Listening to the radio & 216 & 23.0 & 54.2 & 45.8 & 1.31 & .252 \\
or recordings & & & & & & \\
\hline
\end{tabular}

appearance (such as physical attractiveness: $n=123,13.1 \%$ ) or emotional state $(n=171,18.2 \%)$.

When comparing the type of self-descriptions cited according to gender (idem Table 2), it is observed that men make more mention of intellectual characteristics $\left(\chi^{2}=4.16, p=.041\right)$ while women are in the majority when referring to preferences $\left(\chi^{2}=16.14, p=.000\right)$.

Table 2. Self-descriptions. Prevalence according to gender

\begin{tabular}{l|r|r|r|r|r|r}
\hline & \multicolumn{2}{|c|}{$\begin{array}{c}\text { Total sample } \\
(N=938)\end{array}$} & \multicolumn{2}{c|}{ Gender } & & \\
\hline Self-descriptions & \multicolumn{1}{c|}{$n$} & \multicolumn{1}{c|}{$\%$} & $\begin{array}{c}\text { Men } \\
(n=476)\end{array}$ & $\begin{array}{c}\text { Woman } \\
(n=462)\end{array}$ & \multicolumn{1}{c}{$\chi^{2}$} & \multicolumn{1}{c}{$p$} \\
\hline Intellectual & 285 & 30.4 & 55.8 & 44.2 & 4.16 & .041 \\
Practical & 237 & 25.3 & 53.6 & 43.4 & 1.02 & .312 \\
Character/moral & 739 & 78.8 & 50.2 & 49.8 & .41 & .521 \\
Social & 638 & 68.0 & 50.0 & 50.0 & .44 & .505 \\
Appearance & 123 & 13.1 & 51.2 & 48.8 & .01 & .910 \\
Emotional & 171 & 18.2 & 46.8 & 53.2 & 1.31 & .252 \\
Preferences & 533 & 56.8 & 45.0 & 55.0 & 16.14 & .000 \\
\hline
\end{tabular}


The presence of one type of self-description or another also shows significant associations depending on the leisure activities practiced (Table 3). Thus, character or moral attributes are mentioned by those active in entertainment and culture $\left(\chi^{2}=4.43, p=.035\right)$, and art and hobbies $\left(\chi^{2}=2.87, p=.090\right)$. The expression of preferences is habitual among those who participate more in social life $\left(\chi^{2}=3.56, p=.059\right)$, watch TV, DVD or videos $\left(\chi^{2}=4.87, p=.027\right)$ and listen to the radio or recordings $\left(\chi^{2}=5.71, p=.017\right)$. Other significant associations observed reveal that the practice of an activity may be associated with the absence of any reference to certain self-descriptions. Specifically, those who enjoy the leisure activities of reading $\left(\chi^{2}=4.01, p=.045\right)$ and listening to the radio or recordings $\left(\chi^{2}=6.77, p=.009\right)$ tend to ignore self-descriptions concerning appearance. For their part, those who do physical exercise are those who most avoid self-descriptions of their emotional outlook $\left(\chi^{2}=\right.$ $3.29, p=.070)$.

\section{Discussion}

Overall, the research findings provide new insights into young adults' leisure activities and identities. At the same time, they illustrate the potential of the TBT and TST, used independently and in combination. These findings are specific on four aspects: the characterization of young people's leisure activities as serious or casual leisure; the main traits that make up youth identity; the associations between leisure activities and self-descriptions; and the gender differences displayed in the practice of leisure activities and in the descriptions that people provide of themselves.

Among the eight leisure activities mentioned by the participants -and following Stebbins (1992, 1997, 2006)- three are classified as serious leisure (physical exercise, art and hobbies, computer use) being casual leisure the remaining five (social life, entertainment and culture, reading, watching television, DVDs or videos, listening to the radio or recordings).

As has been observed, casual leisure activities (social life and watching television, DVDs or videos) are the ones practiced by larger proportions of the sample. As far as serious leisure activities (physical exercise and computer use) are concerned, these are practiced by approximately fifteen per cent of the sample. Regarding young people's identity, the most frequent self-descriptions focus on the attitudinal categories dealing with character (or morals) and social life.

Leisure activities and self-descriptions of identity are related in two complementary ways. On the one hand, there are activities whose practice is specifically associated with certain self-descriptions; and, on the other hand, it seems 


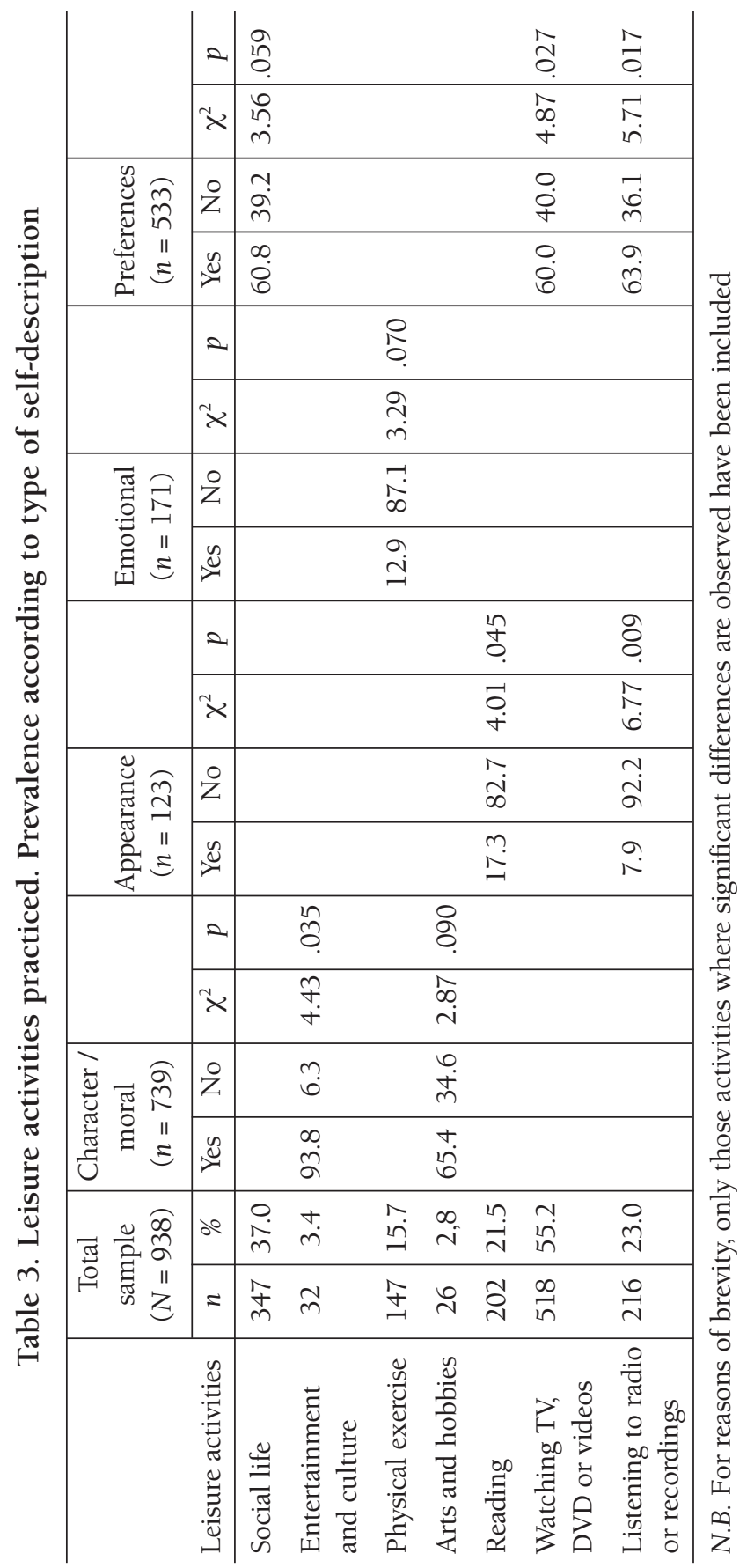


that certain self-descriptions are played down on taking up a leisure activity. With regard to the leisure activities under consideration, it has been observed that those who practice serious leisure activities such as arts and hobbies focus their self-descriptions on character and moral traits, while an activity such as physical exercise minimizes the importance of emotional outlook. People whose leisure activities fall into the casual category tend to express their preferences and describe their characters and morals; likewise, there are casual leisure activities that play down the self-description of appearance. Among casual leisure activities, listening to the radio or recordings deserves special mention, since those who practice this activity state their preferences but minimize the relevance of self-descriptions of appearance.

One of the findings that will not leave those researching the relationship between leisure and identity indifferent is the verification of the differences between men and women -both in relation to leisure activities and self-descriptions of identity. Preferences about activities and the prevalence of certain self-descriptions fuel the debate on the differences between men and women in terms of leisure and identity, in the light of serious and casual leisure in this case.

\section{CONCLUSIONS}

Young people's leisure activities and identity have been researched using methodologies and theories that had not previously been applied conjointly. To be precise, new and relevant information on the relationship between leisure and youth identity has been obtained through qualitative methodologies where the participants felt free to express themselves. But the novelty of this methodological proposal is twofold. On the one hand, it works a qualitative approach with quantitative rigor, which is reflected both in the preparation of materials and the software for collecting data, as well as in the analysis of the results -in which an attempt is made to minimize the inferences derived from the subjectivity of the researcher. On the other hand, the procedure carried out reveals relationships and knowledge that in other methodological approaches imply reductions, given the simplicity of the reality that introduce the structured questionnaires tests or highly codified answers analysis.

As regards the contributions of this research to the existing corpus of knowledge, we have studied the relationships between leisure activities and identities by means of consolidated theoretical frameworks such as the Stebbins's Serious Leisure Perspective and Kuhn and McPartland's analysis of subconsensual self-descriptions. In this aspect -and unlike many studies in which it is 
argued that leisure is fundamental in the construction of identities- this work shows explicitly and empirically how serious leisure and casual leisure dialogue with the construction of identity.

As regards the significant associations between certain leisure activities and attitudinal self-descriptions, it is observed that while casual leisure is generally linked to self-descriptions of preferences, serious leisure activities such as hobbies are significantly associated with moral self-descriptions, but not significantly associated with self-descriptions of physical activity and emotional outlook. Therefore, as well as revealing significant associations between activities and self-descriptions, it emerges that the activities that demand more commitment -and the activities that do not- are linked to different attitudinal aspects of the self. These findings draw attention to the complementary roles played by serious leisure and casual leisure in the construction of plural identities -those that better protect and strengthen the person- (Codina \& Pestana, 2008; Linville, 1987).

The results also confirm an established fact and one of its effects. We are referring to the differences in leisure practices determined by gender, and their repercussions on the male and female processes of identity. This finding is important, because, to the usual differences in the practice of certain activities, it is shown that these differences go beyond reflecting the construction of identity. Moreover, it enables us to understand other differences that are related to the temporal attitudes (like Time Perspective or Procrastination: Codina $\&$ Pestana, 2016). The methodology and theory applied in this study reveal promising relationships that demand deeper research. In this respect, it is necessary to analyze the evaluations made by young people about those leisure activities where they differ from each other, as well as their perceptions of identity. The latter is an example of the wide range of stimulating questions and challenges that derive from the findings of this study.

\section{NOTE}

This study was funded by the Ministry of Economy and Competitiveness of Spain conjointly with the European Regional Development Fund (project EDU2012-39080-C07-04). The authors would like to thank Christopher Gladwin for his invaluable help in correcting the English language manuscript. This correction received the financial support of the PsicoSAO-Research Group in Social, Environmental, and Organizational Psychology (2014 SRG 992; Secretaria d'Universitats i Recerca del Departament d'Economia i Coneixement, Generalitat de Catalunya). 


\section{REFERENCES}

American Psychological Association (2010). Publication Manual of the American Psychological Association (Sixth Ed). Washington, DC: Author.

Barber, B.L., Abbot, B.D., Neira, C.D., \& Eccles, J.S. (2013). Meaningful activity participation and positive youth development. En M. J. Furlong, R. Gilman, E. S. Huebner (eds.), Handbook of Positive Psychology in Schools (2a ed.) (227-244). New York, NY: Routledge.

Burke, P.J. (1991). Identity process and social stress. American Sociological Review, 56(6), 836-849.

Coatsworth, J.D., Sharp, E.H., Palen, L., Darling, N., Cumsille, P., \& Marta, E. (2005). Exploring adolescent self-defining leisure activities and identity experiences across three countries. International Journal of Behavioral Development, 29(5), 361-370.

Codina, N. (1986). Problemática del tiempo libre y preadolescencia [Problems of free time and preadolescence]. Unpublished degree dissertation. University of Barcelona.

Codina, N. (1998). Autodescripción del self en el TST: posibilidades y límites [Self's self-description in the TST: Possibilities and limitations]. Psicologia E Sociedade, 10, 23-38.

Codina, N. (1999). Tendencias emergentes en el comportamiento de ocio: El ocio serio y su evaluación [Arising new tendencies in leisure behavior: Serious Leisure and its evaluation]. Revista de Psicología Social, 14, 331-346.

Codina, N. \& Pestana, J. V. (2008). Investigación del ocio y del self desde el paradigma de la complejidad. Una aplicación de la técnica del presupuesto de tiempo y del Twenty-Statement Test [An investigation of self and leisure from the Complexity Paradigm. A conjoint application of the Time Budget Technique and the TwentyStatement Test]. In E. Aguilar Gutiérrez (Ed.), El influjo del tiempo en la vivencia del ocio. Transformaciones, oportunidades y riesgos en la sociedad apresurada (pp. 135147). Universidad de Deusto. Bilbao.

Codina, N., \& Pestana, J. V. (2009). Análisis psicosocial del comportamiento en el tiempo y sus aspectos complejos mediante la técnica de los presupuestos de tiempo [Psychosocial analysis of behavior over time and its complex elements through Time Budget Technique]. In J. Tous Pallarés y J. M. Fabra Sopeña (Eds.), Actas del XI Congreso Nacional de Psicología Social, vol. I (pp. 57-63). Tarragona: Universitat Rovira i Virgili.

Codina, N. \& Pestana, J. V. (2012). Estudio de la relación del entorno psicosocial en la práctica deportiva de la mujer [Study of the relationships between the psychosocial environment and the woman's sport]. Revista de Psicología del Deporte, 2 Vol 21(2), p. 243-251.

Codina, N., \& Pestana, J.V. (2016). Actividad físico-deportiva como experiencia de ocio y Perspectiva Temporal en los jóvenes [Physical and sport activities and Time Perspective in the youth]. Revista de Psicología del Deporte, 25, suplemento 2, pp. 53-60. 
Codina, N., Pestana, J.V. Balaguer, I. \& Castillo, I. (2016). Ellas a estudiar y bailar, ellos a hacer deporte: Un estudio de las actividades extraescolares de los adolescentes mediante los presupuestos de tiempo ["Girls, study and dance; boys, play sports!" A study of the extracurricular activities of adolescents through time budgets]. Cuadernos de Psicología del Deporte, 16, 1, 233-242.

Eccles, J. S., \& Barber, B. L. (1999). Student council, volunteering, basketball, or marching band: What kind of extracurricular involvement matters? Journal of Adolescent Research, 14, 10-43.

Elkington, S. \& Stebbins, R. A. (2014). The serious leisure perspective: An introduction. Abingdon, UK: Routledge.

Erikson, E. H. (1968). Identity: Youth and crisis. New York: Norton.

Escobar, M., Montes, E., \& Sánchez-Sierra, M. (2015). Sentido, referencia y atribución en las descripciones personales. Una nueva perspectiva del TST [Sense, reference and attribution in self-description. A new TST outlook]. Revista Española de Sociología, 24: 25-41.

Escobar, M. (1983). La autoidentidad. Problemas metodológicos del "Twenty Statements Test" [The self-identity. Methodological problems of the "Twenty-Statement Test"]. Revista Española de Investigaciones Sociológicas, 23: 35-51.

EUROSTAT (2009). Harmonised European Time Use Surveys, 2008 Guidelines. Disponible en: http://ec.europa.eu/eurostat/ramon/statmanuals/files/KS-RA-08-014-EN.pdf

Fredricks, J. A., Alfeld-Liro, C. J., Hruda, L. Z., Eccles, J. S., Patrick, H., \& Ryan, A. M. (2002). A qualitative exploration of adolescents' commitments athletics and the arts. Journal of Adolescent Research, 17, 68-97.

Green, B. C., \& Jones, I. (2005). Serious leisure, social identity and sport tourism. Sport in Society, 8(2), 164-181.

Hansen, D. M., Larson, R. W., \& Dworkin, J. B. (2003). What adolescents learn in organized youth activities: A survey of self-reported developmental experiences. Journal of Research on Adolescence, 13, 25-55.

Henderson, K. A. \& Shaw, S. M. (2006). Leisure and Gender: Challenges and opportunities for feminist research. En C. Rojek, A. Veal , and S. Shaw (eds.) Handbook of Leisure Studies (pp. 216-230). Londres: Routledge.

Hutchinson, S. L. \& Kleiber, D. A. (2005): Gifts of the ordinary: Casual leisure's contribution to health andwell being, World Leisure, 47(3), 2-16.

INE (2015). Padrón municipal 2015. Datos a nivel nacional, comunidad autónoma y provincia [2015 Municipal Census Register. Data from national, autonomous communities and province stratification]. Madrid: Instituto Nacional de Estadística. Disponible en: http://www.ine.es/inebmenu/mnu_cifraspob.htm.

Kleiber, D. A. (1999). Leisure experiences and human development: A dialectical interpretation. New York: Basicbooks.

Kleiber, D. A., Walker, G., \& Mannell, R. C. (2011). A social psychology of leisure (2nd ed.). State College, PA: Venture.

Kuhn, M. H. y McPartland, T. S. (1954), An empirical investigation of self-attitudes. American Sociological Review, 19, 68-76. 
Larson, R. W. (2000). Toward a psychology of positive youth development. American Psychologist, 55, 170-183.

Linville, P. (1987). Self complexity as a cognitive buffer against stress-related illness and depression. Journal of Personality and Social Psychology, 52, 663-676.

MacCosham, B. \& Gravelle, F. (2016) Impact of casual leisure on serious leisure experiences and identity in a Canadian junior hockey context, Leisure/Loisir, 40:3, 325-343, DOI: 10.1080/14927713.2016.1252939

Mahoney, J. L. \& Stattin, H. (2000). Leisure activities and adolescent antisocial behavior: The role of structure and social context. Journal of Adolescence, 23, 113-127.

Mahoney, J. L., Stattin, H., \& Lord, H. (2004). Unstructured youth recreation centre participation and antisocial behaviour development: Selection influences and the moderating role of antisocial peers. International Journal of Behavioral Development, 28, 553-560.

Miller, P. S., \& Kerr, G. (2002). The athletic, academic, and social experiences of intercollegiate student-athletes. Journal of Sport Behavior, 25(4), 346-367.

Morales, J. F. (1989). Identidad social y personal [Social and personal identity]. In. A. Rodríguez \& J. Seoane (Eds.). Creencias, actitudes y valores (pp. 41-87). Madrid: Alhambra.

Neulinger, J. (1986). What am I doing? New York, NY: The Leisure Institute.

Oh, S.-S., Sohn, Y.-M., Shin, K-L, \& Oh, K.-A. (2012). The study for the psychological functioning of casual leisure: Compared with serious leisure. Korean Journal of Physical Education, 51(3), 273-284.

Shin, K. and You, S. (2013). Leisure Type, Leisure Satisfaction and Adolescents' Psychological Wellbeing. Journal of Pacific Rim Psychology, 7(2), 53-62. doi: 10.1017/prp.2013.6.

Silbereisen, R. K., \& Todt, E. (1994). Adolescence in context: The interplay of family, school, peers, and work in adjustment. New York: Springer.

Stebbins, R. A. (1992). Amateurs, Professionals, and Serious Leisure. Montreal and Kingston: McGill-Queen's University Press.

Stebbins, R. A. (1997). Casual leisure: A conceptual statement. Leisure Studies, 16(1), 17-25. doi:10.1080/026143697375485

Stebbins, R. A. (2005). Choice and experiential definitions of leisure. Leisure Sciences, 27, 349-352.

Stebbins, R. A. (2006). Extending the serious leisure perspective. In S. Elkington, I. Jones, \& L. Lawrence, (Eds.). Serious leisure: Extensions and applications (pp. 14). Eastbourne, UK: LSA Publications, University of Brighton.

Stebbins, R. A. (2015). Leisure and positive psychology: Linking activities with positiveness. New York, NY: Palgrave Macmillan.

Stebbins, R.A. (2001). New Directions in the Theory and Research of Serious Leisure. Mellen Studies in Sociology, vol. 28. Lewiston: Edwin Mellen.

Verma, S., \& Larson, R. (Eds.) (2003). Examining adolescent leisure time across cultures: Developmental opportunities and risks. New Directions in Child and Adolescent Development, No. 99. San Francisco: Jossey-Bass. 
Waterman, A. S. (1990). Personal expressiveness: Philosophical and psychological foundations. The Journal of Mind and Behavior, 11, 47-73.

Werthner, P., \& Orlick, T. (1986). Retirement experiences of successful olympic athletes. International Journal of Sport Psychology, 17(5), 337-363.

NURIA CODINA (Department of Social Psychology and Quantitative Psychology, University of Barcelona, Barcelona, Spain. OcioGune Network. ORCID: 0000-0003-0280-3651.E-mail: ncodina@ub.edu)

Received her PhD in 1990 in Psychology from the University of Barcelona. Her psychosocial research on leisure, free time, self, and identity is part of the study of time management, personal well-being and optimal development. In 1999, she published the first analysis of the serious leisure perspective (SLP) addressed to the Spanish language scientific community. She has been visiting scholar in universities of Europe, North and South America.

JOSÉ V. PESTANA (Department of Social Psychology and Quantitative Psychology, University of Barcelona, Barcelona, Spain. OcioGune Network.

ORCID: 0000-0002-8329-0650. E-mail: jvpestana@ub.edu)

Received his PhD in 2007 in Psychology from the University of Barcelona. His dissertation about leisure, self, and theatrical activities -supervised by N. Codina-, obtained the Summa Cum Laude distinction. Visiting scholar in the University of Georgia (Athens, GA) and the University of Sichuan (Chengdu, China), he is currently working in the symbolic aspects of leisure and self from the perspective of Analytical Psychology.

ROBERT A. STEBBINS (Department of Sociology, University of Calgary, Calgary, Canada. ORCID: 0000-0002-6192-355X. E-mail: stebbins@ucalgary.ca) Received his PhD in 1964 in sociology and psychology from the University of Minnesota. His specialty for the past 45 years has been the study of leisure, during which he pioneered the SLP. His most recent book is Leisure's Legacy: Challenging the Common Sense View of Free time. He is currently writing a synthesis of the SLP and supporting empirical work. He is an elected Fellow of the Royal Society of Canada.

Recibido: 06/04/2017

Aceptado: 16/09/2017 


\title{
LA EXPERIENCIA DE OCIO EN FAMILIA DE JÓVENES ESTUDIANTES DE ENSEÑANZA SECUNDARIA POSTOBLIGATORIA FAMILY LEISURE-TIME EXPERIENCES OF POST-COMPULSORY SECONDARY EDUCATION STUDENTS
}

\author{
Joseba Doistua Nebreda \\ Universidad de Deusto, España \\ joseba.doistua@deusto.es \\ Yolanda Lázaro Fernández \\ Universidad de Deusto, España \\ yolanda.lazaro@deusto.es \\ Concepción Bru Ronda \\ Universidad de Alicante, España \\ concha.bru@ua.es
}

\section{Cómo citar / Citation}

Doistua Nebreda, Joseba; Lázaro Fernando, Yolanda y Bru Ronda, Concepción (2017) "La experiencia de ocio en familia de jóvenes estudiantes de enseñanza secundaria postobligatoria". OBETS. Revista de Ciencias Sociales, 12(Extra 1): pp-pp. 81-102. doi:10.14198/OBETS2017.12.1.13

\section{Resumen}

Este artículo pretende resaltar la importancia de las experiencias de ocio compartidas con la familia, entre otros, como ámbito de desarrollo juvenil, tomando en consideración la principal actividad de ocio de la juventud. Se analiza de manera comparada el grupo que realiza dicha actividad en familia, frente a aquellos que la realizan con otras personas, en relación al tipo de prácticas, beneficios percibidos, su vida familiar y la importancia que estas dan al ocio de sus hijos e hijas. Se realiza a través de la explotación de un cuestionario, con una muestra de jóvenes que cursan enseñanzas secundarias postobligatorias. Palabras clave: Ocio, Juventud, Familia, Beneficios del ocio. 


\begin{abstract}
:
The aim of the present article is to emphasise the importance of leisure-time experiences shared with the family, among others, as an area of youth development, considering the principal leisure-time activity of the youth. A group doing the previously mentioned activity with the family and others doing it with other people are analysed in a comparative manner. Regarding the type of the activities, the benefits, their family life and the significance all these have in the leisure time of their children. This information is obtained using a survey with a sample taken from Post-compulsory Secondary Education youth.
\end{abstract}

Key words: Leisure time, Youth, Family, Benefits of leisure.

\title{
Extended abstract:
}

Family is the first place of socialization and construction of identity. According to Espada, González and Campos (2009), the family environment together with the school is where healthy habits are conformed and acquired in our youth. We are currently experiencing profound and convulsive changes in this institution that makes these new models and forms of articulation lead to an openness to family heterogeneity in composition, size (Valdemoros et al., 2014), and in relationships with others institutions. The contemporary family evolves according to the new realities, since it is a plural and dynamic institution. Several authors affirm that family influences in a very decisive way in the construction of the youth leisure, noting that the family's internal functioning is one of the most relevant determinants and equally young people mention the family as an important reference in their vital life.

In this article we understand the leisure experience as a free, satisfying human experience with an end in itself; that is, voluntary and separate from necessity. The Youth 2010 Report points out that the importance of leisure was quite or very important for $90 \%$ of young people, showing an upward trend in the value of free time or leisure. Therefore, leisure has become one of the aspects that gives value and meaning to the youth's life, where they develop skills, aptitudes and values that bring them closer to the feeling of self-fulfillment. It is essential to emphasize that at this stage the peer group or friendships determine to a great extent their leisure practice, beyond the determinations resulting of their family or school practices.

Therefore, overall, this article aims to analyse the importance of leisure-time experiences shared with the family, among others, as an area of youth development. To do so, the principal leisure-time activity of the youth (males and females) is considered, analysing in a comparative manner a group doing the previously mentioned activity with the family and others doing it with other people.

The universe of the subject of study consisted of all the Post-compulsory Secondary Education students in Spain. For the definition of the sample a stratified probability sampling was made (by means of proportional allocation) to the students of Post-compulsory Secondary Education of the General Education System of public, Public Private Partnership and private educational centres 
of Spain (Bachillerato, middle-grade and basic Vocational Trainings). The final sample consists of 2694 surveys ( 34 more than the expected), that allows a sampling error of $1,9 \%$ and a confidence level of $95 \%$. Furthermore, the opinion of the families of the students regarding similar matters was taken into account. The students themselves gathered the surveys and were subsequently brought to the educational centres. 637 surveys were recovered from the families, a $23,6 \%$ of the student body, although it resulted in an unequal distribution on the territorial level.

Keeping the objectives and hypothesis of the study in mind, two surveys were created ad hoc in order to know the distribution of academic and leisure time. One of the surveys was student oriented and the other was directed to their families. Since the subject and its family was clearly identified, both surveys were gathered in the same data base, considering the student body with the main subject as the subject of study.

First of all, with the purpose of identifying the sample subject of study, as well as the most common leisure time activities done with the family, a frequency analysis was made the using Chi-square test to check the relations between both items. Subsequently, the medians were compared using the Student's t test for independent samples and the effect size was determined based on the d coefficient of Cohen (Lakens, 2013). This was calculated for the purpose of describing the implications that participating in leisure time activities with the family has on the level of satisfaction of the youth and the importance families confer to the leisure time activities of their children, comparing the ones thinking leisure time should be spent with the family and those who differ. As well as to compare which benefits are obtained from the previously mentioned activities and the opinion they have about their family life.

The youths doing their principal leisure time activity with their families represent a $13,8 \%$ of the total sample. Concerning their age, although the 1618 year-olds form the biggest group, the percentage of those doing their leisure time activities with their families is higher in the cohort of 19-21 and 22-24 year-olds, 9 percentage points and 2 percentage points respectively. As for gender, the percentage of women doing the principal leisure time activity with their family is significantly higher than that of the man.

The main discoveries and implications of this study are as follows: the main leisure activities carried out with the family refer to collecting, animal care, plant care, shopping, etc., and festive leisure, that is, to help in the organization of parties and celebrations, as well as going out to have a drink.

In the case of sports leisure, although it is a leisure activity that starts or is promoted within the family (Anuario de Estadísticas Deportivas, 2016), in the juvenile stage turns out to be one of the most activities carried out without the family.

Finally, in terms of leisure activities, another block can be defined by those that are carried out in front of a screen (digital leisure or television), which mean more than $22 \%$ in the case of practicing without the family, compared to $6.8 \%$ in the case of making them in family. 
Regarding the satisfaction with the activity and the importance of this in its life, it is concluded that in both groups (young people who carry out their main activity of leisure with the family and those who do not made with it) there are esteemed high averages. However, satisfaction with the activity presents higher values than the values that indicate the importance of the activity in the life of the young person.

In this same line, can be made an interpretation of the perception of the benefits that the main practice of leisure provides to the young people. This is also corroborated with the data found in the analysis according to the type of benefits, being those of an emotional nature (satisfaction and enjoyment) those that present the highest values in both groups.

As regards to their family life, found data allow to affirm that for the young population family is important.

\section{INTRODUCCIÓN}

\section{La familia en la sociedad actual}

Los últimos cuarenta años han sido testigos, según Valdivia (2008), de los cambios más profundos y convulsivos de la familia en los últimos veinte siglos.

La familia es una realidad que no puede entenderse exclusivamente desde las estadísticas puesto que comprende conjuntas y variadas relaciones de vivencias e interacciones personales que son difícilmente cuantificables (Navarro, 2006). Es una estructura social con identidad propia y con roles diferenciados (Bas y Pérez de Guzmán, 2010).

De hecho puede decirse que se hace difícil llegar a una definición que sea capaz de reconocer la variedad de modelos existentes, los cuales pueden depender (Valdivia, 2008: 19):

- De su estructura, puesto que hoy la familia puede suponer eliminación o modificación de subsistemas tradicionales tales como conyugal, parental, filial y fraternal.

- De su funcionamiento, dada la modificación de los papeles del hombre y la mujer en el hogar.

- De la educación, por los estilos educativos y los valores que se viven y transmiten en relación al tipo de familia.

Todos estos nuevos modelos y formas de articulación conllevan una apertura a la heterogeneidad familiar tanto en su composición, en su tamaño (Valdemoros y otros, 2014), como en las relaciones con otras estructuras socioinstitucionales (escuela, iglesia, servicios sociales, etc.). La familia actual evoluciona de acuerdo con las nuevas realidades, es una institución plural y dinámica. 
Pero con todo ello, la institución familiar sigue teniendo un gran aprecio social y sigue siendo valorada como un pilar fundamental de la vida personal y social (Álvarez y Rodríguez, 2008).

\section{El ocio en el contexto familiar}

La familia es el primer lugar de socialización y de construcción de la identidad. En opinión de Espada, González y Campos (2009) el ámbito familiar junto con el escolar es donde se conforman y adquieren hábitos saludables en nuestros jóvenes, por lo que será importante y necesario un trabajo coordinado y complementario.

Asimismo, Valdemoros y otros (2014) afirman que la familia influye de una manera muy decisiva en la construcción del ocio juvenil, señalando que el funcionamiento interno familiar es uno de los condicionantes más relevantes. Se apunta que el rol materno-paternal se sitúa en un lugar distinguido en la construcción adecuada del ocio de los hijos y las hijas. El núcleo familiar educa y socializa, por ello ha de revisar sus capacidades, valores, actitudes y normas de convivencia para transmitir una sociabilidad adecuada a los/las niños/as y jóvenes (Valdemoros y otros, 2011).

Diversas investigaciones (Martínez-Pampliega, Iraurgi y Sanz, 2011) señalan tres constructos fundamentales que ayudan a la explicación y comprensión del contexto idóneo para la construcción de un ocio entendido como experiencia de desarrollo humano y crecimiento personal. Por un lado la cohesión, entendida como la reciprocidad emocional que puede darse entre los miembros de una familia; la flexibilidad como la capacidad para adecuarse a los cambios y adaptaciones que pueden requerirse y la comunicación como el acto de dar a conocer a todos los miembros de la familia las informaciones que deben compartir.

Según investigaciones realizadas por el Grupo de Investigación PSICOSOC (2011) las y los jóvenes citan a la familia como un referente importante en su existencia vital, apuntando que su valoración hacia ella ha ido en aumento a medida que ellos han ido madurando. Igualmente han señalado que comparten valores apuntados por las personas adultas como son la responsabilidad, disciplina, compromiso, educación, motivación y exigencia. En definitiva, las personas jóvenes valoran positivamente a la familia sin olvidar la relevancia que otorgan al grupo de iguales como referente clave en la formación de su identidad y en la vivencia de su ocio.

González-Anleo y González (2010) en su Informe Jóvenes españoles 2010 concluyen que la juventud considera "muy importante", ante todo, su familia, la salud y los amigos. También señalan que las relaciones padres/madres - hijos/as 
han mejorado considerablemente en los últimos años convirtiéndose en un lugar privilegiado tanto de gestación de ideas e interpretaciones del mundo para el joven como de búsqueda de apoyo y de consejo. Al mismo tiempo, encuentran en la familia su primer y fundamental espacio de libertad y autonomía, debido al talante negociador de la familia española.

Educar poniendo en valor la importancia del ocio para el bienestar de las personas, forma parte, en opinión de Caride (2012) de las tareas pedagógica y socialmente provechosas.

\section{Experiencia de ocio satisfactorio joven}

En las últimas décadas del pasado siglo XX, se han producido profundas y significativas transformaciones políticas, económicas, sociales y culturales que han supuesto cambios estructurales de enorme importancia, tanto para las personas consideradas individualmente, como para los grupos sociales de los que forman parte. Estos cambios, en opinión de Bru (2010) han alterado las condiciones de vida, de trabajo, de relación y de ocio de las personas de todas las edades.

El ocio ha pasado de ser considerado exclusivamente un medio a ser un fin en sí mismo, contribuyendo su satisfacción a una mejor calidad de vida y bienestar de la ciudadanía. En este artículo entendemos la experiencia de ocio (Cuenca, 2000) como una vivencia humana libre, satisfactoria y con un fin en sí misma; es decir, voluntaria y separada de la necesidad. Igualmente defendemos el Derecho al Ocio como un Derecho Humano que debe ser garantizado puesto que favorece el desarrollo personal y comunitario (Lázaro, 2006).

Caride (2012: 309), señala que "las experiencias de ocio, en su inscripción en los nuevos tiempos sociales y los riesgos que comportan en una sociedad espacio-temporal transformada, están siendo objeto de un profundo debate intelectual y social".

No se puede olvidar la importancia del presente que domina la mentalidad actual. Hay autores que hablan de la urgencia de las gratificaciones inmediatas (Torío, 2004) y Lipovestky (1994), máximo exponente de la postmodernidad, apunta que estamos bajo el imperio de lo efímero. Así, para Cuenca $(2011,2014)$ lograr que la vivencia de ocio sea una experiencia valiosa implica cambios conceptuales, actitudinales y estructurales, y supone asumir que, frente a un ocio consumo, resulta imprescindible trabajar un ocio experiencial. Por lo tanto, actualmente, el ocio se ha convertido en uno de los aspectos que otorga valor y sentido a la vida. Por ello, en relación a sus prácticas, se viene insistiendo en la hipótesis de que éstas ocupan un lugar muy relevante para los aprendizajes y las experiencias de la infancia, la adolescencia y la juventud (Monteagudo, 2008), siendo la participación de la educación y los/las educadores/as imprescindible. 
El Informe Jóvenes 2010 señala que la importancia del ocio era bastante o muy importante para el $90 \%$ de jóvenes, mostrando una tendencia alcista del valor del tiempo libre o del ocio en la última década (López, 2010), que junto a otros valores vinculados a la estética o la sexualidad, refuerzan la matriz presentista y hedonista características de las sociedades postmodernas (Rodríguez, 2010).

Según las conclusiones del sondeo de opinión "Jóvenes, ocio y consumo" realizado por el INJUVE (2014), las actividades de ocio que resultan más atractivas para los y las jóvenes españoles/as, son: usar el ordenador, hacer deporte, ir de excursión, ir al teatro, ir a museos, exposiciones, asistir a conferencias, coloquios. Por el contrario, ir a discotecas, a bailar y ver la televisión son las actividades que más atractivo pierden.

En cuanto al ocio practicado y teniendo en cuenta que las oportunidades de ocio de los y las jóvenes se ven limitadas por un descenso del dinero disponible para llevar a cabo ciertas prácticas, se apunta que las que crecen significativamente, en comparación con los datos de 2007 de que disponía el INJU$\mathrm{VE}$, son practicar y hacer deporte, viajar, leer libros y usar el ordenador.

Investigaciones anteriores como la presentada por Doistua, Cuenca y Ortega (2014) muestran cómo la juventud apunta que más allá de su simple práctica, actividades tales como práctica deportiva, pasear, estar con las amistades, o navegar por internet, tienen gran importancia en su vida puesto que suponen una experiencia seria (Stebbins, 2008) a la cual le atribuyen importantísimos beneficios psicológicos. Además, diferencian perfectamente estas experiencias serias de otras prácticas más casuales o que no tienen una significación tan elevada para el propio joven. Es en la diferencia de las prácticas señaladas en un caso u otro lo que permite a estos autores afirmar que la actividad no es lo que define la experiencia de ocio, sino que el joven dota a esta experiencia de valores diferentes en función de sus necesidades de desarrollo personal y social.

También es importante destacar que la democratización del ocio ha supuesto un importante aprecio hacia el mismo por parte de las personas jóvenes y de la población en su conjunto a pesar de los impresionantes datos en relación al paro en general y al paro juvenil en particular. Incluso en esta situación, el valor del ocio no pierde sino que gana enteros.

En opinión de Robinson y Arounica (2009) el ocio puede ser una fuente importante de sentido de vida donde los y las jóvenes desarrollen habilidades, aptitudes y valores que les aproxime al sentimiento de realización personal. En este trabajo, la juventud es entendida como un espacio social de experimentación individual prolongado, donde conviven una multiplicidad de situaciones 
(Gil, 2005; Tejerina, Carbajo y Martínez, 2012). En esta etapa vital, se añade el hecho de que el grupo de pares o amistades determinan en gran medida su práctica de ocio, más allá de las determinaciones devenidas de sus prácticas familiares o escolares (Doistua, Cuenca y Ortega, 2014). Además, la juventud piensa que estar en red es imprescindible para mantener una vida social plena (Espinar y González, 2009).

Otras investigaciones (Valdemoros y otros, 2011), estiman que la familia como agente educativo puede influir en la juventud tanto en el inicio como en el mantenimiento de algunas prácticas de ocio, como son las físico-deportivas, e igualmente pueden mediar en la no adhesión y abandono de las mismas.

En esta dirección, el Anuario de Estadísticas Deportivas ( $4^{a}$ ed., 2016), señala que entre los y las españolas de 15 o más años que practican deporte, el 34,2\% afirma que al menos uno de sus progenitores practica o ha practicado esta actividad. Igualmente el 41,6\% de la población con hijos/as menores de 18 años afirma que realiza con ellos/as alguna práctica deportiva. Igualmente el $42,2 \%$ suele acompañar a sus hijos/as a sus entrenamientos y el $35,2 \%$ a sus competiciones.

Es por todo esto que en este texto se plantean los siguientes objetivos de trabajo:

- Describir sociodemográficamente la muestra de alumnado que practica la principal actividad de ocio con la familia frente a aquellos que la practican sin ella.

- Especificar las diferentes modalidades de ocio de la principal actividad que señala el alumnado en función de la compañía con la que la realizan.

- Analizar las implicaciones que la realización de actividades de ocio con la familia tiene en el grado de satisfacción que las personas jóvenes perciben y la importancia que otorgan a las mismas, frente a aquellas actividades que realizan con otras personas.

- Identificar los beneficios percibidos de la práctica de ocio en función de la compañía, teniendo en cuenta que estos beneficios pueden ser de carácter físico, emocional, cognitivo, conductual o social.

- Mostrar la opinión del alumnado en relación a su vida familiar, así como la importancia que las familias confieren al ocio de sus hijos e hijas.

\section{METODOLOGÍA}

El trabajo de investigación que da lugar a este artículo fue posible gracias a la colaboración de diferentes universidades del estado español, que participaron activamente tanto en el diseño del instrumento, como en el trabajo de campo. A continuación se destacan los aspectos más importantes a este respecto. 


\section{Participantes}

El universo objeto de estudio lo componían todas las personas jóvenes que estaban cursando Enseñanzas Secundarias Postobligatorias en el estado español. Como prueba piloto se realizó una primera aplicación del cuestionario a 140 jóvenes de ocho comunidades autónomas diferentes, que permitió modificar y adaptar la herramienta previamente a la aplicación definitiva de la misma de manera masiva.

Para la definición de la muestra se optó por realizar un muestreo probabilístico estratificado (mediante una afijación proporcional) al conjunto de la población de estudiantes de Enseñanza Secundaria Postobligatoria de régimen general de centros públicos, concertados y privados del territorio español (Bachillerato, Formación Profesional de Grado Medio y Formación Profesional Básica). A la hora de realizar las afijaciones proporcionales se ha optado por tomar como referencia territorial las llamadas Áreas Nielsen (áreas delimitadas por el instituto de mercados Nielsen), así como el tipo de estudios, la titularidad de los centros educativos y el sexo.

La muestra finalmente obtenida ha sido de 2694 (34 cuestionarios más de los previstos), que permiten un error muestral del 1,9\% y un nivel de confianza del 95\%. En cuanto a la distribución territorial, las divergencias entre la distribución del universo objeto de estudio entre las 10 áreas planteadas y la muestra finalmente obtenida no supera en ningún caso el 3\%, por lo que no se realizaron correcciones.

Además, se tomó en consideración la opinión de las familias de dicho alumnado respecto a temas similares, mediante una encuesta que recogía el propio alumnado y que posteriormente eran remitidas a los centros educativos. Se estimó una tasa de respuesta en torno al 40\%, que finalmente resultó ser inferior, ya que pudieron recuperarse 637 , lo que supone un $23,6 \%$ del alumnado. Además, hubo una distribución desigual a nivel territorial, por lo que se entiende que la información obtenida puede ser valiosa, sobre todo en análisis que no realicen comparativas a nivel territorial, como es en este caso.

Atendiendo al objetivo principal de esta investigación, las principales características sociodemográficas se muestran teniendo en cuenta los que realizan su actividad principal de ocio con o sin la compañía de sus familias (13,8\% y $86,2 \%$ respectivamente). Así pues, tal y como se observa en la tabla 1 , la mayoría de las respuestas son de jóvenes entre 16 y 18 años tanto entre los que más realizan sus prácticas de ocio con sus familias como los que no la realizan con la familia, contemplando porcentajes menores a medida que aumenta la edad. Aun así, el porcentaje, en el caso de aquellos que realizan la actividad principal con la familia, es mayor que el porcentaje de aquellos que no realizan esa activi- 
dad con la familia, tanto en la cohorte de 19 a 21 años ( 9 puntos porcentuales) como en la de 22 a 24 años ( 2 puntos porcentuales).

En cuanto al sexo, el porcentaje de mujeres que realizan la actividad principal de ocio con la familia es sustancialmente superior al porcentaje de hombres, mientras que en el caso de las que lo realizan sin la familia es menor el número de mujeres que el de hombres. En el caso del tipo de estudios, es en los ciclos formativos de grado medio donde se encuentran mayores diferencias, al ser mayor la presencia de alumnado que practican la actividad de ocio con la familia, frente a los que la realizan sin ella. Por último, señalar que la titularidad del centro no interfiere en que los y las jóvenes practiquen ocio con su familia o no.

Tabla 1. Principales características sociodemográficas de la muestra

\begin{tabular}{|c|c|c|c|c|c|c|c|c|}
\hline & \multirow{2}{*}{$\begin{array}{c}\text { Total } \\
\mathrm{N} \\
\end{array}$} & \multicolumn{2}{|c|}{ Con familia } & \multicolumn{2}{|c|}{ Sin familia } & \multirow[b]{2}{*}{$\chi^{2}$} & \multirow[b]{2}{*}{$p$} \\
\hline & & & $\mathrm{n}$ & $\%$ & $\mathrm{n}$ & $\%$ & & \\
\hline \multirow[t]{2}{*}{ Sexo } & Hombre & 1382 & 157 & 42,3 & 1225 & 52,9 & \multirow{2}{*}{14,25} & \multirow{2}{*}{$\leq, 001$} \\
\hline & Mujer & 1306 & 214 & 57,7 & 1092 & 47,1 & & \\
\hline \multirow[t]{3}{*}{ Edad } & Entre 16 y 18 años & 1740 & 204 & 63,0 & 1536 & 74,3 & \multirow{3}{*}{22,25} & \multirow{3}{*}{$\leq, 001$} \\
\hline & Entre 19 y 21 años & 608 & 108 & 33,3 & 500 & 24,2 & & \\
\hline & Entre 22 y 24 años & 42 & 12 & 3,7 & 30 & 1,5 & & \\
\hline \multirow{3}{*}{$\begin{array}{l}\text { Tipo de } \\
\text { estudios }\end{array}$} & Bachillerato & 1752 & 209 & 56,2 & 1543 & 66,5 & \multirow{3}{*}{19,88} & \multirow{3}{*}{$\leq, 001$} \\
\hline & Ciclo formativo de & 714 & 123 & 33,1 & 591 & 25,5 & & \\
\hline & FPB & 228 & 40 & 10,8 & 188 & 8,1 & & \\
\hline \multirow{3}{*}{$\begin{array}{l}\text { Titularidad } \\
\text { centros }\end{array}$} & Pública & 2073 & 276 & 76,0 & 1797 & 79,0 & \multirow{3}{*}{2,17} & \multirow{3}{*}{,338 } \\
\hline & Privada laica & 120 & 21 & 5,8 & 99 & 4,3 & & \\
\hline & Privada religiosa & 446 & 66 & 18,2 & 380 & 16,7 & & \\
\hline
\end{tabular}

$X^{2}=$ Prueba de Ji cuadrado; $p=$ valor de significación

\section{Instrumento}

Atendiendo a los objetivos e hipótesis de la investigación se elaboraron dos cuestionarios ad hoc para conocer la organización de los tiempos académicos y de ocio, uno orientado al alumnado y otro a las familias de los mismos. Dado que estaba perfectamente identificado el sujeto y su familia, se agruparon ambos cuestionarios en una misma base de datos, considerando al alumnado como el sujeto principal objeto de estudio. Teniendo en cuenta la complejidad de los cuestionarios, este artículo se centra en algunas de las secciones de los mismos, la dedicada al tiempo libre y a la vida familiar, concretamente, en las siguientes variables: 
Actividad de ocio más importante que practican realizada con la compañía de la familia (variable independiente): el alumnado tenía que indicar la actividad de ocio más importante que realiza de un listado de 49 posibilidades. Estas actividades se presentaron agrupadas en 9 modalidades: Televisión y radio, ocio digital, ocio deportivo, turismo y excursionismo, ocio cultural, ocio festivo, juegos (sin ordenador), otras actividades y ocio solidario. Para la elaboración de este artículo se tomó en consideración dicha práctica principal de ocio en relación a con quién la practican (sólo, con su familia, con gente de su edad y/o, con otras personas), para poder obtener aquellos sujetos que realizan dicha práctica con la familia, frente a los que la realizan sin su compañía.

Satisfacción con las actividades de ocio (variables dependientes): a través de una escala Likert con gradación de 5 puntos (nada, poco, algo, bastante, mucho), el alumnado indicó su nivel de acuerdo con las siguientes afirmaciones: me siento más satisfecho con esta actividad y esta actividad es muy importante en mi vida.

Beneficios percibidos de la práctica de ocio (variables dependientes): de nuevo, a través de una escala de Likert con una gradación de 5 puntos, el alumnado valora los beneficios percibidos de la práctica de dicha actividad en función de que estos sean beneficios físicos, emocionales, cognitivos, conductuales y sociales.

Vida familiar (variables dependientes): en escala de Likert con gradación de 5 puntos se solicita al alumnado que se posicione en torno a diferentes cuestiones que hacen referencia a la opinión que tienen sobre su vida familiar.

Importancia que las familias otorgan al ocio de sus hijos/as (variable dependiente): en una única pregunta, se solicita a la persona que completa el cuestionario para la familia que valoren del 1 al 5 la importancia que otorgan al ocio de sus hijos/as.

\section{Procedimiento}

Tras un primer contraste entre expertos y una prueba piloto, se definió la necesidad, debido a la complejidad del cuestionario, de que dos investigadores se desplazaran a cada centro educativo para la aplicación de los cuestionarios en el aula. Tras el contacto y la transmisión de los objetivos del estudio a los centros educativos, se bloquearon las aulas necesarias y se contó con la colaboración del profesorado de dichas aulas para la aplicación de los cuestionarios. El alumnado participó en la investigación de forma voluntaria y anónima, siendo informado de los objetivos de la investigación y con el conocimiento y consentimiento de las familias y de los equipos directivos de los centros. Asimismo, se remitió a las familias un cuestionario para que lo cumpli- 
mentasen de manera voluntaria en sus propios domicilios y que fueron remitidos al centro educativo.

\section{Análisis de datos}

En un primer lugar, con el fin de identificar cuáles son las modalidades de ocio más practicadas en compañía de la familia, se realizó un análisis de frecuencias recurriéndose a la prueba Ji-cuadrado para comprobar la relación entre éstas. Posteriormente, para describir las implicaciones que la realización de actividades de ocio con la familia tiene en el grado de satisfacción de las personas jóvenes y la importancia que las familias confieren al ocio de sus hijos e hijas, comparando si difieren en función de ser prácticas compartidas por la familia o no, así como para comparar los beneficios percibidos de dichas actividades y la opinión sobre su vida familiar, se realizó un contraste de medias mediante la prueba $t$ de Student para muestras independientes, así como el tamaño del efecto a partir del coeficiente $d$ de Cohen (Lakens, 2013).

\section{RESULTADOS}

Los resultados se agrupan en función de los objetivos planteados, por lo tanto, en primer lugar se presentan los datos de las frecuencias de las principales modalidades de ocio practicadas; en segundo lugar, el grado de satisfacción y la importancia que otorgan a dicha actividad de ocio; en tercer lugar, la percepción de beneficios al realizar dichas actividades; en cuarto lugar, la opinión que tienen sobre su vida familiar y; en quinto y último lugar, la importancia que las familias otorgan al ocio de sus hijos/as.

El análisis de frecuencias mostró que las actividades de ocio más significativas para las personas jóvenes que cursan Enseñanza Secundaria Postobligatoria en España son: ocio deportivo (33,4\%), ocio cultural (17,2\%), ocio digital $(14,1 \%)$, ocio festivo $(13,3 \%)$, y otras actividades $(12,6 \%)$ entre ellas, coleccionar cosas o cuidar animales. Estas cinco modalidades agrupan al 86,6\% de la muestra. El 13,4\% practica otro tipo de modalidades de ocio como turismo y excursionismo u ocio solidario.

En función de realizar estas actividades con o sin sus familias se detallan los resultados en la tabla 2. De este modo, el 36\% de los y las jóvenes realiza con su familia otras actividades, como cuidar animales, ir de compras, cuidar plantas (huertos), manualidades, coleccionismo, etc., un porcentaje significativamente mayor al del alumnado que practica dichas actividades sin su familia. Lo mismo sucede con el ocio festivo (31\%), donde se encuentran actividades tales como salir a tomar algo o ayudar en la organización de fiestas y celebraciones.

Las prácticas que menos realizan las personas jóvenes, tanto con sus familias como sin ellas son turismo y excursionismo, juegos sin ordenador y ocio 
solidario. Y las prácticas de ocio donde es mayor el porcentaje de actividad que se realiza sin familia son ocio digital, deportivo o cultural.

Tabla 2. Principales modalidades de ocio practicadas en función de la compañía de la familia

\begin{tabular}{|c|c|c|c|c|c|c|c|}
\hline & \multicolumn{2}{|c|}{ Con familia } & \multicolumn{2}{|c|}{ Sin familia } & \multirow[b]{2}{*}{$\chi^{2}$} & \multirow[b]{2}{*}{$p$} \\
\hline & & $\mathrm{n}$ & $\%$ & $\mathrm{n}$ & $\%$ & & \\
\hline \multirow{9}{*}{$\begin{array}{l}\text { Modalidades } \\
\text { de ocio }\end{array}$} & TV y radio & 7 & 1,9 & 156 & 6,8 & \multirow{9}{*}{412,62} & \multirow{9}{*}{$\leq, 001$} \\
\hline & Ocio digital & 18 & 4,9 & 361 & 15,6 & & \\
\hline & Ocio deportivo & 43 & 11,6 & 858 & 37,2 & & \\
\hline & Turismo y excursionismo & 1 & 0,3 & 50 & 2,2 & & \\
\hline & Ocio cultural & 47 & 12,7 & 423 & 18,3 & & \\
\hline & Ocio festivo & 116 & 31,3 & 242 & 10,5 & & \\
\hline & Juegos (sin ordenador) & 0 & 0,0 & 7 & 0,3 & & \\
\hline & Ocio solidario & 4 & 1,1 & 7 & 0,3 & & \\
\hline & Otras actividades & 135 & 36,4 & 205 & 8,9 & & \\
\hline
\end{tabular}

$X^{2}=$ Prueba de Ji cuadrado; $p=$ valor de significación

En relación al grado de satisfacción con la práctica y la importancia de la misma en la vida del alumnado (tabla 3), se observan medias elevadas en ambos casos (tanto las realizadas con la familia, como las realizadas sin su presencia), aunque significativamente mayores en el caso de aquellas actividades realizas sin la familia. Esta diferencia está presente tanto en el caso del grado de satisfacción con la actividad como en el caso de la importancia que dicha actividad tiene para el alumnado. En este segundo caso, con mayor diferencia.

Tabla 3. Contraste de medias en la compañía de la práctica de ocio respecto a la satisfacción e importancia de la actividad

\begin{tabular}{l|c|c|c|c|c|c|c}
\hline & \multicolumn{2}{|c|}{$\begin{array}{c}\text { Con familia } \\
(\mathrm{n}=372)\end{array}$} & \multicolumn{2}{l|}{$\begin{array}{c}\text { Sin familia } \\
(\mathrm{n}=2322)\end{array}$} & \multicolumn{4}{l|}{} \\
\hline & $\mathrm{M}$ & $\mathrm{DE}$ & $\mathrm{M}$ & $\mathrm{DE}$ & $\mathrm{t}$ & $\mathrm{p}$ & $\mathrm{d}$ \\
\hline Satisfacción con la actividad & 4,40 & 1,04 & 4,58 & 0,84 & $-3,70$ & $\leq, 001$ &, 21 \\
\hline $\begin{array}{l}\text { Importancia de la actividad } \\
\text { en su vida }\end{array}$ & 3,89 & 1,31 & 4,26 & 1,09 & $-5,90$ & $\leq, 001$ &, 33 \\
\hline
\end{tabular}

$T=t$ de student; $p=$ valor de significación 
Atendiendo a la tabla 4 que presenta los datos relativos a la percepción de los beneficios que las prácticas de ocio realizadas en compañía de la familia o sin su compañía, son perceptibles diferencias significativas en todos los casos, excepto en el de los beneficios cognitivos que hacen referencia a la creatividad y la adquisición de conocimientos, siendo en este caso medias similares aunque ligeramente superior en el caso de realizarlas en familia.

En el resto de beneficios percibidos, siempre resultan inferiores las medias en aquellas actividades realizadas en familia. Las medias más bajas, en todo caso, hacen referencia a los beneficios físicos (estar más en forma, controlar mejor los movimientos, mantener o mejorar la condición física, etc.) y los beneficios conductuales (desarrollar nuevas destrezas manuales y adquisición o perfeccionamiento de habilidades técnicas). En cambio, la media más elevada se encuentra en el caso de los beneficios emocionales (satisfacción realizando la práctica, disfrute o diversión, etc.). Los beneficios sociales (hacer cosas diferentes con más gente, sentirse parte de un grupo, mejorar la manera de relacionarse con los demás, etc.) se sitúan en segundo lugar en el caso de si se realizan sin la familia y en tercer lugar en el caso de que se realice con la familia.

En relación a la vida familiar, las medias que se presentan en la tabla 5 señalan datos positivos. En cambio, si bien de manera general, se considera que la vida familiar es buena (en prácticamente todos los casos las medias superan

Tabla 4. Contraste de medias en la compañía de la práctica de ocio respecto a la percepción de beneficios al realizar las actividades de ocio

\begin{tabular}{l|c|c|c|c|c|c|c}
\hline & \multicolumn{2}{|c|}{$\begin{array}{c}\text { Con familia } \\
\text { (n=372) }\end{array}$} & \multicolumn{2}{c|}{$\begin{array}{c}\text { sin familia } \\
(\mathrm{n}=2322)\end{array}$} & \multicolumn{2}{|l}{} \\
\hline & $\mathrm{M}$ & $\mathrm{DE}$ & $\mathrm{M}$ & $\mathrm{DE}$ & $\mathrm{t}$ & $\mathrm{p}$ & $\mathrm{d}$ \\
\hline $\begin{array}{l}\text { 1. Beneficios físicos: Estar más } \\
\text { en forma }\end{array}$ & 2,68 & 1,63 & 3,10 & 1,68 & $-4,49$ & $\leq, 001$ &, 25 \\
\hline $\begin{array}{l}\text { 2. Beneficios emocional: } \\
\text { Satisfacción y disfrute }\end{array}$ & 4,37 & 1,02 & 4,59 &, 84 & $-4,54$ & $\leq, 001$ &, 25 \\
\hline $\begin{array}{l}\text { 3. Beneficios cognitivos: } \\
\text { Creatividad y conocimiento }\end{array}$ & 3,74 & 1,24 & 3,66 & 1,28 & 1,12 &, 261 &, 06 \\
\hline $\begin{array}{l}\text { 4. Beneficios conductual: } \\
\text { Destrezas y habilidades }\end{array}$ & 2,88 & 1,52 & 3,28 & 1,58 & $-4,55$ & $\leq, 001$ &, 25 \\
\hline $\begin{array}{l}\text { 5. Beneficios sociales: } \\
\text { Relación con otras personas }\end{array}$ & 3,33 & 1,57 & 3,86 & 1,41 & $-6,62$ & $\leq, 001$ &, 36 \\
\hline
\end{tabular}

$T=t$ de student; $p=$ valor de significación 
valores de 3,5 sobre 5), aquel alumnado que comparte las actividades de ocio con sus familias presenta medias superiores. En el caso de la consideración de que la mayoría de las veces su vida familiar es ideal; que las condiciones de su vida familiar sean excelentes; que si pudieran decidir sobre su vida familiar no cambiarían nada y; que si pudieran, algún día les gustaría tener su propia familia, las medias en ambos grupos no presentan diferencias significativas.

En cambio, en el caso del grado de satisfacción general y con la consecución de objetivos importantes con su vida familiar, sí que se presentan medias significativamente superiores en el caso de realizar la principal práctica o actividad de ocio en familia frente a realizarlas en soledad o con sus grupos de iguales.

Tabla 5. Contraste de medias en la compañía de la práctica de ocio respecto a la vida familiar

\begin{tabular}{l|c|c|c|c|c|c|c}
\hline & \multicolumn{2}{|c|}{$\begin{array}{c}\text { on familia } \\
(\mathrm{n}=372)\end{array}$} & \multicolumn{2}{|c|}{$\begin{array}{c}\text { Sin familia } \\
(\mathrm{n}=2322)\end{array}$} & \multicolumn{4}{l}{} \\
\hline & $\mathrm{M}$ & $\mathrm{DE}$ & $\mathrm{M}$ & $\mathrm{DE}$ & $\mathrm{t}$ & $\mathrm{p}$ & $\mathrm{d}$ \\
\hline Vida familiar ideal & 3,69 & 1,05 & 3,54 & 1,09 & 2,47 &, 013 &, 13 \\
\hline Condiciones excelentes & 3,69 & 1,03 & 3,59 & 1,07 & 1,68 &, 093 &, 09 \\
\hline Grado de satisfacción & 4,15 & 1,02 & 3,96 & 1,07 & 3,20 &, 001 &, 19 \\
\hline He conseguido cosas importantes & 3,78 & 1,08 & 3,61 & 1,12 & 2,73 &, 006 &, 15 \\
\hline No cambiaría nada & 3,48 & 1,34 & 3,38 & 1,34 & 1,34 &, 181 &, 08 \\
\hline Me gustaría tener familia propia & 4,37 & 1,09 & 4,26 & 1,13 & 1,75 &, 080 &, 10 \\
\hline
\end{tabular}

$T=t$ de student; $p=$ valor de significación

Finalmente (tabla 6), en relación a la importancia que otorgan las familias al ocio de sus hijos e hijas, en el caso de aquellas familias cuyos hijos/as practican su principal actividad de ocio con ellos/as es significativamente menor a las que

Tabla 6. Contraste de medias en la compañía de la práctica de ocio respecto a la importancia que dan las familias al ocio de sus hijos/as

\begin{tabular}{l|c|c|c|c|c|c|c}
\hline & \multicolumn{2}{|c|}{$\begin{array}{c}\text { Con familia } \\
(\mathrm{n}=75)\end{array}$} & \multicolumn{2}{|c|}{$\begin{array}{c}\text { Sin familia } \\
(\mathrm{n}=513)\end{array}$} & \multicolumn{4}{|l}{} \\
\hline & $\mathrm{M}$ & $\mathrm{DE}$ & $\mathrm{M}$ & $\mathrm{DE}$ & $\mathrm{t}$ & $\mathrm{p}$ & $\mathrm{d}$ \\
\hline $\begin{array}{l}\text { Importancia al ocio de sus } \\
\text { hijos/as }\end{array}$ & 3,89 & 0,81 & 4,04 & 0,80 & $-3,35$ &, 001 &, 18 \\
\hline
\end{tabular}

$T=t$ de student $; p=$ valor de significación 
no lo practican con su familia. En ambos casos, la importancia que las familias otorgan al ocio de sus hijos e hijas es elevado, rondando el cuatro en una escala de uno a cinco.

\section{DISCUSIÓN}

Tras el análisis presentado en base a los objetivos planteados, se puede concluir que las principales prácticas de ocio realizadas con la familia hacen referencia al coleccionismo, cuidado de animales, cuidado de plantas, ir de compras, etc., y al ocio festivo, es decir, ayudar en la organización de fiestas y celebraciones, así como salir a tomar algo. Por lo tanto, por un lado, actividades que se pueden compartir con el grupo de amistades y con la familia y, por otro lado, actividades relacionadas con el hogar y la vida familiar, que trabajan valores, actitudes y normas de convivencia tal y como señalan Valdemoros y otros (2011). Igualmente, a través de estas actividades se puede trabajar la cohesión, flexibilidad, comunicación, responsabilidad, disciplina, compromiso, u otros valores, constructos fundamentales para el desarrollo juvenil.

En cambio, en el caso del ocio deportivo, si bien es una actividad de ocio que se inicia o promueve dentro del ámbito familiar (Anuario de Estadísticas Deportivas, 2016), en la etapa juvenil resulta ser una de las actividades más realizadas sin la familia (solo, en compañía del grupo de pares o en la escuela), alcanzando el 37,2\% del total de actividades, frente al 11,6\% del grupo que realiza dichas actividades con la familia. Por tanto, la actividad deportiva, vistos los resultados del análisis como otras investigaciones realizadas (INJUVE, 2014), se trata de la actividad más practicada por la población joven.

Finalmente, en cuanto a las actividades de ocio, otro bloque lo pueden conformar aquellas que se realizan delante de una pantalla (ocio digital o televisión) que suponen más de un $22 \%$ en el caso de practicarlas sin la familia, frente al 6,8\% en el caso de realizarlas en compañía familiar. Esto puede corroborar la idea presentada por Espinar y González (2009) que afirman que la juventud piensa que para mantener una vida social plena es imprescindible estar en red, y esa puede ser una razón que lleve a que este tipo de actividades la muestren como su principal práctica de ocio, casi uno de cada cinco jóvenes, y que sean actividades compartidas con su grupo de pares y no con su familia.

En cuanto a la satisfacción con la actividad y la importancia de esta en su vida, se concluye que en ambos colectivos (jóvenes que realizan su principal actividad de ocio con la familia y los que no la realizan con ella) se aprecian medias elevadas. Ahora bien, la satisfacción con la actividad presenta valores más elevados (4,40 en el caso del colectivo que realiza las actividades en familia y 4,58 en el colectivo que las realiza sin la familia), que los que señalan la impor- 
tancia de la actividad en la vida del joven ( 3,89 y 4,26 respectivamente). Esto puede llevar a pensar que la satisfacción o gratificación inmediata de la actividad es el núcleo central de la vivencia del ocio joven (Lipovestky, 1994; Torío, 2004). Trabajar para que esta vivencia sea una experiencia valiosa es una tarea pendiente para familia, educadores/as y las propias personas jóvenes con el objeto de que perciban la importancia y potencialidad que el ocio tiene en su desarrollo personal. Las diferencias en estos ítems que se aprecian entre el colectivo de jóvenes que practican la actividad principal con la familia y sin ella, pueden deberse principalmente a la tipología de actividades de ambos colectivos, ya que en esta etapa vital el grupo de pares o amistades determinan en gran medida su práctica de ocio (Doistua, Cuenca y Ortega, 2014).

En esta misma línea, se puede hacer la lectura de la percepción de los beneficios que la principal práctica de ocio proporciona a las personas jóvenes. Esto se confirma, además, con los datos encontrados en el análisis según la tipología de beneficios, siendo los de carácter emocional (satisfacción y disfrute) los que presentan los valores más elevados en ambos colectivos $(4,37$ en compañía familiar y 4,59 sin compañía familiar). Les siguen, en el colectivo que realiza las prácticas sin la familia, con una media de 3,86 los beneficios sociales (relación con otras personas), mientras que los y las jóvenes que realizan su práctica principal de ocio con la familia señalan los beneficios cognitivos $(3,74)$, creatividad y conocimiento, en segundo lugar. Este tipo de beneficios cognitivos ya se apuntaban en el informe Jóvenes españoles 2010 donde se señalaba la familia como un lugar destacado para la creación de ideas e interpretaciones del mundo.

Destaca como el valor más bajo en cuanto a la percepción de beneficios que proporciona la práctica de ocio más realizada sea, para ambos colectivos (con y sin compañía familiar), los beneficios físicos (2.68 y 3,10 respectivamente) lo que lleva a pensar que aun siendo esta práctica deportiva una de las más realizadas en el colectivo joven, no es la búsqueda de estar en forma el objetivo de dicha práctica, sino posiblemente la búsqueda de relaciones y el disfrutar de un espacio de socialización y diversión.

En cuanto a su vida familiar, se observan datos que permiten afirmar que para la población joven la familia es importante. Las medias descritas en el análisis en referencia a la opinión que las personas jóvenes tienen en cuanto a su vida familiar son en todos los casos elevadas, lo que apoya la idea transmitida por Álvarez y Rodríguez (2008) en cuanto a que la familia sigue teniendo un gran aprecio social y es valorada como un pilar fundamental de la vida personal y social. Además el informe Jóvenes españoles 2010 señalaba que la población joven destaca el talante negociador de la familia actual, ya que consideran 
que la familia es también un espacio fundamental de libertad y autonomía. Realizando una lectura comparativa de ambos grupos (los que realizan su actividad principal de ocio con la familia y los que no), se observa que de las seis afirmaciones analizadas en relación a la opinión sobre su vida familiar, en todos los ítems, las medias son ligeramente superiores en el caso de realizar la actividad en familia, si bien solo en uno de ellos, el que hace referencia al nivel general de satisfacción con su vida familiar, es significativamente mayor.

Finalmente, en el análisis de los datos que dan cuenta de la importancia que las familias otorgan al ocio de sus hijos/as, se aprecia un nivel superior en aquellas familias que no comparten la práctica principal de ocio con ellos/as, si bien resultan medias elevadas muy cercanas al valor cuatro sobre cinco, en ambos casos. La diferencia del valor superior que presentan las familias que no comparten la actividad principal con las personas jóvenes puede deberse a un mayor desconocimiento o preocupación por el uso del tiempo libre y de las prácticas de ocio de sus hijos/as. Ahora bien, en ambos casos los datos permiten afirmar que las familias entienden y valoran el ocio como un elemento importante en la vida y desarrollo personal de las personas jóvenes.

\section{CONCLUSIONES}

Existe una preocupación evidente por parte de la sociedad en general y de las familias en particular por la utilización del tiempo libre y las prácticas de ocio desarrolladas por la juventud. Muchas investigaciones ponen de manifiesto la importancia del ocio en el desarrollo juvenil. Este artículo ahonda en las prácticas de ocio de la población joven analizando la significación que estas tienen cuando son compartidas con uno de los agentes de socialización más importantes como es la familia.

Posiblemente el hecho de realizar la actividad de ocio principal con la familia determina el tipo de práctica, destacando como las más realizadas el cuidado de animales, de plantas, ir de compras, preparar celebraciones y fiestas. En cambio, fuera del ámbito familiar destaca el ocio deportivo y digital. Cada una de estas actividades determina el grado de satisfacción e importancia que la juventud otorga a su ocio, así como los beneficios que perciben de su práctica.

Si bien la familia posee una influencia importante en la configuración de los hábitos de ocio de sus hijos/as, se observa que en la etapa juvenil, las prácticas de ocio se realizan principalmente fuera de este ámbito, sobre todo en el caso de la actividad principal de ocio que es aquella más significativa para la población joven. Sería interesante analizar el resto de actividades de ocio que estas personas jóvenes desarrollan como complemento a los datos que se presentan en este artículo. 
Otro indicador de los cambios que se dan en la etapa juvenil tiene que ver con la relación entre actividad y tiempo, por tanto, una línea de investigación a futuro podría ser un análisis de la distribución de las actividades de ocio realizadas a lo largo del día y de la noche y la compañía con la que se realizan. Igualmente, los tiempos y espacios escolares y vacacionales también podrían aportar diferencias significativas en el papel de la familia en el ocio de sus hijos/as.

Analizar si lo hobbies o las consideradas prácticas de ocio serio de las personas jóvenes han estado condicionadas por las prácticas de ocio compartidas en familia, pudiera ser un trabajo de interés para complementar los datos que se aportan en este artículo.

En definitiva, hablar de ocio, juventud y familia es un campo extenso de posibilidades. Este artículo permite afirmar que las personas jóvenes no dejan de ver la institución familiar, con sus distintos modelos, como un referente y un pilar fundamental en su vida personal y social.

\section{BIBLIOGRAFÍA}

Álvarez, J. y Rodríguez, C. (2008). El valor de la institución familiar en los jóvenes universitarios de la Universidad de Granada. Bordón, 60, 1, 7-21.

Bas, E. y Pérez de Guzmán, M.V. (2010). Desafíos de la familia actual ante la escuela y las tecnologías de información y comunicación. Educatio Siglo XXI, vol. 28, núm. $1,42-68$.

Bru, C. (2010). La enseñanza universitaria para personas mayores: evolución conceptual, innovación educativa y adaptación a las demandas sociales del siglo XXI. Investigación y buenas prácticas de trabajo académico con mayores en España. Palabras Mayores, 5.

http://ezproxybib.pucp.edu.pe/index.php/palabrasmayores/article/view/1601/1553

Caride, J.A. (2012). Lo que el tiempo educa: el ocio como construcción pedagógica y social. ARBOR. Ciencia, Pensamiento y Cultura, 188, marzo-abril, 301-313.

Cuenca, M. (2000). Ocio humanista. Documentos de Estudios de Ocio, núm. 16. Bilbao: Universidad de Deusto.

Cuenca, M. (2011). Aproximación a las experiencias culturales desde los planteamientos del Ocio Humanista: pautas para una profundización cualitativa. En C. Ortega (Ed.), Nuevos desafíos de los observatorios culturales. Bilbao: Universidad de Deusto, $19-48$.

Cuenca, M. (2014). Ocio valioso. Documentos de Estudios de Ocio, núm. 52. Bilbao: Universidad de Deusto.

Doistua, J.; Cuenca, M. y Ortega, C. (2014). Prácticas y experiencias de ocio en los jóvenes de la comunidad autónoma de Euskadi. En G. Pérez Serrano y A. De-Juanas Oliva (Coords.), Educación y jóvenes en tiempos de cambio. Madrid: UNED, 375-386. 
Espada, M.; González, M.D. y Campos Izquierdo, A. (2009). Familia y actividades físico-deportivas extraescolares. Padres y Maestros, núm. 323, 5-7.

Espinar, E. y González, M.J. (2009). Jóvenes en las redes sociales virtuales. Un análisis exploratorio de las diferencias de género. Feminismo/s, 14, diciembre, 87-106.

Gil, E. (2005). El envejecimiento de la juventud. De Juventud, núm. 71, 9-11.

González-Anleo, J. y González, P. (2010). Jóvenes Españoles 2010. Madrid: Fundación SM.

Grupo de Investigación PSICOSOC. Centro de Estudios Superiores Don Bosco. (2011). El sentido de vida en los jóvenes: redes sociales, relaciones significativas y actividades de ocio. Revista de Estudios de Juventud, 95, 9-72.

INJUVE (2014). Conclusiones. Sondeo de opinión. Jóvenes, ocio y consumo. Madrid: Secretaría de Estado de Servicios Sociales e Igualdad. http://www.injuve.es/observato rio/ocio-y-tiempo-libre/jovenes-ocio-y-consumo

Lakens, D. (2013). Calculating and reporting effect sizes to facilitate cumulative science: A practical primer for t-tests and ANOVAs. Frontiers in Psychology, 4:863. doi:10.3389/fpsyg.2013.00863.

Lázaro, Y. (2006). Derecho al Ocio. En M. Cuenca (Coord.), Aproximación multidisciplinar a los Estudios de Ocio. Documentos de Estudios de Ocio, núm. 31. Bilbao: Universidad de Deusto, 143-156.

Lipovetsky, G. (1994). El imperio de lo efímero. La moda y su destino en las sociedades modernas. Barcelona: Anagrama.

López, J.A. (2010). Ocio, consumo y medios de comunicación. En J. González-Anleo y P. González (Dir./Coord.), (2010). Jóvenes españoles 2010. Madrid: Fundación SM, 229-298.

Martínez-Pampliega, A.; Iraurgi, I. y Sanz, M. (2011). Validez estructural del FACES20 Esp: Versión española de 20 ítems de la Escala de Evaluación de la Cohesión y Adaptabilidad Familiar. Revista Iberoamericana de Diagnóstico y Evaluación Psicológica, 29, 1, 147-165.

Ministerio de Educación, Cultura y Deporte. (2016). Anuario de estadísticas deportivas 2016. Madrid: Secretaría General Técnica.

Monteagudo, $\mathrm{M}^{\mathrm{a}} \mathrm{J}$. (Ed.). (2008). La experiencia de ocio: una mirada científica desde los Estudios de Ocio. Documentos de Estudios de Ocio, núm. 35. Bilbao: Universidad de Deusto.

Navarro, L. (2006). Modelos ideales de familia en la sociedad española. Revista Internacional de Sociología (RIS), vol. LXIV, núm. 43, enero-abril, 119-138.

Robinson, K y Arunica, L. (2009). The element. New York: Penguin Group USA.

Rodríguez, E. (2010). Socialización y referentes socio-grupales en la construcción de la identidad juvenil. Educación y Futuro, (22), 55-69.

Stebbins, R.A. (2008). Serious leisure: a perspective for our time. New Brunswick: Transaction

Tejerina, B.; Carbajo, D. y Martínez, M. (2012). El fenómeno de las lonjas juveniles. Nuevos espacios de ocio y sociabilidad en Vitoria-Gasteiz. Informes del CEIC, 004.

Torío, S. (2004). Familia, escuela y sociedad. Aula Abierta, 83, 35-52. 
Vademoros, M.A.; Ponce de León, A.; Ramos, R. y Sanz, E. (2011). Pedagogía de la convivencia y educación no formal: un estudio desde el ocio físico-deportivo, los valores y la familia. European Journal of Education and Psychology, vol. 4, núm. 1, 33-49.

Vademoros, M.A.; Ponce de León, A.; Sanz, E. y Caride, J.A. (2014). La influencia de la familia en el ocio físico-deportivo juvenil: nuevas perspectivas para la reflexión y la acción. ARBOR. Ciencia, Pensamiento y Cultura, 190-770, noviembre-diciembre, 1-11. doi: http://dx.doi.org/10.3989/arbor.2014.770n6013

Valdivia, C. (2008). La familia: concepto, cambios y nuevos modelos. La Revue du REDIF, vol. 1, 15-22.

DR. JOSEBA DOISTUA NEBREDA. Instituto de Estudios de Ocio. Facultad de Ciencias Sociales y Humanas. Universidad de Deusto.

joseba.doistua@deusto.es Código ORCID: 0000-0002-8421-9733.

Doctor en Ocio y Potencial Humano y Master en Gestión de Ocio por la Universidad de Deusto. Licenciado en Historia por la UPV/EHU. Doctor acreditado por Unibasq (Agencia de calidad del sistema universitario vasco). Director de DeustoBide, escuela de Ciudadanía y profesor de la Facultad de Ciencias Sociales y Humanas de la Universidad de Deusto. Miembro del equipo de Ocio y Desarrollo Humano dentro del Instituto de Estudios de Ocio.

DRA. YOLANDA LÁZARO FERNÁNDEZ. Departamento de Didáctica y Desarrollo Curricular. Facultad de Psicología y Educación. Universidad de Deusto. yolanda.lazaro@deusto.es Código ORCID: 0000-0002-9059-357X.

Doctora en Ocio y Potencial Humano por la Universidad de Deusto. Master en Gestión de Ocio y Licenciada en Psicología. Ha recibido evaluación positiva de la Agencia de Evaluación de la Calidad y Acreditación del Sistema Universitario Vasco (UNIQUAL). Miembro del Equipo Oficial de Investigación reconocido por el Gobierno Vasco IT984-16 "Ocio y Desarrollo Humano". Reconocimiento A (2016-2021). Actualmente es PDI en la Facultad de Psicología y Educación de la que es docente y coordinadora del Grado en Ciencias de la Actividad Física y Deporte.

DRA. CONCEPCIÓN BRU RONDA. Doctora en Geografía por la Universidad de Alicante. Profesora Titular en el Departamento de Análisis Geográfico Regional. Ha impartido clases en grados, postgrados y masters desde entonces. Ha ocupado diversos cargos académicos: Dir. Escuela Oficial de Turismo de la GV, Vicedecana Facultad de Filosofía y Letras, Dir. Instituto Marítimo Internacional, Dir. Escuela Oficial de Turismo de la UA, Dir. Sede de la Universidad en Alicante, con rango de Vicerrectora, y actualmente Dir. Universidad Perma- 
nente de la UA. Presidenta de la Asociación Estatal de Programas Universitarios para Personas Mayores. Entre las líneas de investigación destaca su pertenencia a los Grupos de: Planificación y gestión sostenible del turismo; Medio, Sociedad y Paisaje (MedSPai); y Educación Universitaria para Mayores.

Recibido: 03/04/2017

Aceptado: 16/09/2017 


\section{SATISFACCIÓN DE LAS PERSONAS JÓVENES CON LAS ACTIVIDADES DE OCIO ENTRE PARES SATISFACTION OF YOUNG PEOPLE WITH LEISURE ACTIVITIES BETWEEN PEERS}

Douglas A. Kleiber

Recreation and Leisure Studies

Program Department

Counseling and Human

Development Services

Universidad de Georgia,

Estados Unidos

dkleiber@uga.edu

Idurre Lazcano

Instituto de Estudios de Ocio

Facultad Ciencias Sociales y Humanas

Universidad de Deusto, España

ilazkano@deusto.es
Aurora Madariaga

Instituto de Estudios de Ocio

Facultad Ciencias Sociales y Humanas

Universidad de Deusto, España

aurora.madariaga@deusto.es

Daniel Muriel

Instituto de Estudios de Ocio

Facultad Ciencias Sociales y Humanas

Universidad de Deusto, España

daniel.muriel@deusto.es

\section{Como citar / Citation}

Kleiber, Douglas A; Lazcano, Idurre; Madariaga, Aurora y Muriel, Daniel (2017) "Satisfacción de las personas jóvenes con las actividades de ocio entre pares". OBETS. Revista de Ciencias Sociales, 12(Extra 1): pp-pp. 103-120. doi:10.14198/OBETS2017.12.1.14

\section{Resumen}

El artículo parte de la hipótesis de que aquellas personas jóvenes que tienen un mayor grado de responsabilidad en la organización de sus actividades de ocio presentan mayores niveles de satisfacción con la práctica, favoreciendo que estas sean actividades relevantes en sus vidas. Los objetivos de este trabajo son: (a) identificar las prácticas de ocio más significativas que tienen lugar 
en el grupo de pares y, (b) analizar si determinadas actividades de ocio entre pares inciden en la satisfacción e identificación de experiencias valiosas, y por último (c) comprobar si las personas jóvenes que participan en la organización de estas experiencias valiosas presentan una mayor satisfacción, disfrute e importancia en las actividades practicadas.

Palabras clave: Ocio; Juventud; Experiencia; Participación.

\section{Abstract}

The article is based on the hypothesis that young people, who have a greater degree of responsibility in the organization of leisure activities, have higher levels of satisfaction with practice, encouraging that these are essential activities in their lives. The main purposes of this work are: (a) to identify the most significant leisure practices that take place in the peer group, and (b) to analyze if certain types of leisure activities in peers affect the satisfaction and identification of valued experiences, and finally (c) to verify, if young people who participate in the organization of these valued experiences, present a greater satisfaction, enjoyment and importance in the activities practiced.

Key Words: Leisure youth; Experience; Participation.

\section{Extended abstract}

Some research results (Hills, Argylea y Reevesb, 2000) show that when adolescents are very interested in leisure activities, their personal satisfaction grows, that is, the degree of motivation of a youngster has a positive influence on leisure satisfaction; on the same way, some results indicate that young people show greater satisfaction with leisure activities in which they acquire greater autonomy and self-management opportunities (Ortega, Lazcano y Baptista, 2015). Thus, young people participate more and have higher degrees of satisfaction in those spaces of leisure that they share with their peer group and in which they assume high levels of self-management and participation. Focusing on existing literature, this article is based on a hypothesis which states that leisure activities self-managed by young people and developed with their peer group can be expected of showing higher levels of satisfaction, reflecting their relevance in their lifestyles. The objectives of this work are: (a) to characterize and typify the most significant leisure practices that take place in the peer group, and (b) to analyze whether certain typologies leisure activities in peers affect the satisfaction and identification of valued experiences, and finally (c) to check whether young people who participate in the organization of these valued experiences show a greater satisfaction, enjoyment and importance in the activities practiced.

The carried out research, from which this article originates, focused on the young people involvement, aged between 16 and 18, in the constitution and management of spaces where they develop their leisure. A stratified probabilistic sampling was selected (by proportional attachment) to the whole population of post-compulsory secondary education students in private and public centers in Spain (General Certificate of Education and Vocational Education and Training). 
According to the objectives and hypotheses of the research, an ad hoc questionnaire was elaborated to know the organization of academic and leisure time. This article focuses on one of the sections of the questionnaire, the one dedicated to leisure (most important leisure activity practiced, satisfaction with leisure activities and organization in leisure activities). When proportional attachment was performed, territorial areas called Nielsen areas were took into account as well as the type of studies, ownership of schools and sex. The sample obtained was 2694 students.

In order to characterize and typify the most significant leisure modalities, a frequency analysis was performed, using the Chi-square test to test the relationship between these and the gender of young people. In relation to the second objective, related to the fact of whether these modalities affect in their satisfaction and vital significance, that is, their effect on the dependent variables, an analysis of the variance (ANOVA) was carried out. The Cohen d (Faul, Erdfelder, Lang y Buchner, 2007) was also calculated in order to know the size of the effect. Finally, the checking of the third objective, to test if the young people who participate further in the organization of these valued experiences are related to a higher satisfaction, enjoyment and importance in the activity was carried out with a correlation analysis between the variables related to organization and satisfaction explained, distinguishing, also, the sex of young people. The significance of the independent effect differences in men and women found for each variable was assessed using the Fisher $\mathrm{Zr}$ transformation and using the Preacher (2002) form.

The obtained results show that the most significant leisure activities for young people, who study Post-compulsory Secondary Education in Spain, are those related to sports leisure, especially in the case of men, and cultural leisure activities in the case of women, hence, gender is a determining factor in the choice of the most significant leisure mode. Regarding the second objective, it is possible to conclude that young people show higher satisfaction with the leisure activity with a solidarity character and that the same young people feel more satisfied and enjoy more developing sport and solidarity practices. With regard to the third objective, the results show that having responsibility in the organization of the activity gives them greater satisfaction, they enjoy more and the activity is more important in their lives. Nevertheless, there are no differences between men and women.

\section{INTRODUCCIÓN}

El contexto económico y sociopolítico, y los rasgos que identifican a la juventud, tienen su reflejo en otros ámbitos. Uno de ellos lo constituyen los espacios y tiempos de ocio, cuya tipología varía dependiendo del grado de autonomía, participación y uso de los mismos, coexistiendo una gradación de prácticas que van del polo de la monitorización, hasta el polo de la autogestión.

Las personas jóvenes practican actividades de ocio como parte de proyectos más amplios de vida, en un contexto social en el que pueden dar forma a sus 
identidades. De hecho, buena parte de la investigación sobre juventud y adolescencia se ha centrado en el ocio y en los modos en que este tiempo se organiza (Lazcano y Madariaga, 2016b). Y, ha sido así, tanto por lo que supone el tiempo libre como espacio-tiempo de socialización, como por constituirse en un escenario particular para la exposición de propuestas y la toma de decisiones (INJUVE, 2012).

Los adolescentes tienen pocas oportunidades para desarrollar su capacidad de decidir, sus tareas típicas durante el trabajo escolar y el ocio estructurado no reúnen las condiciones apropiadas para el aprendizaje de su autonomía (Larson, 2000). El contexto más adecuado para su desarrollo parece ser el de las actividades voluntarias y la participación en organizaciones.

Los resultados de distintas investigaciones sugieren que tales actividades se asocian con el desarrollo positivo en estas franjas de edad (Leversen, Danielsen, Birkeland y Oddrun, 2012; King y Church, 2015), entendiendo el desarrollo (social y emocional) como un proceso madurativo, de crecimiento e incremento de la autonomía. Sin embargo, la capacidad de participación y la autonomía no es un resultado garantizado que derive espontáneamente del tránsito de la infancia a la adolescencia y juventud (Deci y Ryan, 2000). Durante la adolescencia, los individuos tienen una necesidad cada vez mayor de asumir tareas complejas y de implicarse en la toma de decisiones.

En relación con la satisfacción con el ocio, multitud de estudios han indagado sobre los efectos determinantes de su relación con las actividades de ocio y sus componentes. La satisfacción con las prácticas de ocio está muy relacionada con la satisfacción vital, entendida ésta como la percepción de bienestar físico, psicológico y social, muchos autores han revelado que la participación en actividades recreativas contribuye positivamente al bienestar de los adolescentes (Csikszentmihalyi y Hunter, 2003; Onishi, Masuda, Suzuki, Gotoh, Kawamura, y Iguchi, 2006; Sacker y Cable, 2006).

Algunas investigaciones (Hills, Argylea, y Reevesb, 2000) muestran que cuando los adolescentes están muy interesados en las actividades de ocio, su satisfacción personal crece, es decir, el nivel de motivación de un adolescente tiene una influencia positiva en la satisfacción con el ocio. En esta misma línea, algunos resultados señalan que las personas jóvenes manifiestan mayor satisfacción con las actividades de ocio en las que adquieren una mayor autonomía (Moreno y Martínez, 2006; Aibar, Julián, Murillo, García-González, Estrada y Bois, 2015) y oportunidad de autogestión (Ortega, Lazcano y Baptista, 2015), que explicaría que las personas jóvenes participan más y con grados de satisfacción superiores en aquellos espacios de ocio que comparten con su grupo de pares y en los que asumen niveles elevados de autogestión o de participación en la organización. 
El ocio se ha consolidado como un valor claramente en alza, como herramienta de desarrollo y satisfacción con la vida, pero pocos estudios han incorporado la implicación de las y los jóvenes en la organización de sus actividades. Con todo, a pesar de los trabajos existentes, no se han realizado demasiadas investigaciones que exploren, con constructos conceptuales más complejos, la participación de las y los propios jóvenes en la organización de sus experiencias y espacios. Este artículo estudia si la participación en la organización de actividades de ocio está relacionada con los niveles de satisfacción con la práctica, así como el grado de significación de estas actividades en su vida.

En virtud de lo expuesto anteriormente, atendiendo a la bibliografía existente, a modo de hipótesis de partida, puede esperarse que las actividades de ocio autogestionadas por las personas jóvenes y desarrolladas con su grupo de pares muestren niveles más altos de satisfacción, favoreciendo que éstas sean actividades relevantes en sus vidas. Los objetivos de este trabajo son: (a) identificar las prácticas de ocio más significativas que tienen lugar en el grupo de pares, (b) analizar si determinado tipos de actividades de ocio entre pares inciden en la satisfacción e identificación de experiencias valiosas, y por último (c) comprobar si las personas jóvenes que participan en la organización de estas experiencias valiosas presentan mayores niveles de satisfacción y disfrute, otorgando mayor importancia en las actividades practicadas.

\section{REVISIÓN DE LA LITERATURA}

En este apartado del artículo se realiza una breve aproximación conceptual a los temas más estrechamente relacionados con la satisfacción de las personas jóvenes con las actividades de ocio con el grupo de pares, con el objetivo de dotar de un contexto teórico a los datos que posteriormente se presentan y discuten.

Las personas jóvenes conforman un grupo social definido a partir del siglo XIX con el surgimiento de movimientos juveniles de diferente naturaleza cuya característica compartida es la rebeldía y el deseo de transformación, ambos van adquiriendo diferentes formas y estilos en cada generación joven (Elzo y Megias, 2014). Este grupo constituye un segmento de la población cuya razón fundamental es la edad, aunque a veces este criterio operativo es discutido, ya que además de formar grupos estadísticos significativos se infiere que cada subgrupo plantea diferencias significativas entre sí (Alaminos, 2008). De todos modos, indudablemente, a pesar de ser una construcción social (Comas, 2011), constituye un colectivo muy relevante por el papel que va a tener en cada sociedad en un futuro a medio y largo plazo (INJUVE, 2012).

En el plano psicológico, las principales dimensiones del desarrollo juvenil (Gómez-Granell, y Julià, 2015) son: (a) la identidad (autopercepción, auto- 
definición, autoconcepto y caracterización personal estable en el tiempo), (b) la autonomía (el control interno, la competencia frente a los problemas y la capacidad de tomar decisiones), (c) el pragmatismo orientado a la acción, los vínculos (relaciones de apego, capacidad de reconocer, comprender y expresar sentimientos y emociones en las relaciones con los demás) (Rodríguez, 2010), (d) las redes (condiciones sociales y familiares cercanas que ejercen de apoyo a la persona joven), (e) los modelos (situaciones y personas que sirven de guía y referencia), (f) las metas (objetivos definidos, acciones encaminadas hacia un fin y proyección de futuro) y (g) la satisfacción (percepción de logro, autovaloración, percepción de desarrollo, y adaptación efectiva a las condiciones ambientales y al contexto externo) (Larson, 2000). Desde el punto de vista más físico, la juventud es el momento álgido del desarrollo en cuanto a variables tales como: la agilidad, la rapidez y la fortaleza física.

La satisfacción, entendida como el equilibrio entre los afectos positivos y negativos que componen los estados emocionales, es la integración cognitiva de cómo evalúa la vida cada persona y se relaciona estrechamente con la percepción de bienestar (Heintzman, 2000), siendo el bienestar la satisfacción de necesidades en general, más allá de lo meramente psicológico, y el bienestar psicológico una vivencia personal y subjetiva (Elzo y Megias, 2014).

La juventud constituye un periodo en el que, de forma progresiva, la persona se va enfocando, transitando a la vida adulta, relacionado, habitualmente, con la finalización de estudios, la asunción de compromisos de pareja y posteriores adquisiciones de obligaciones familiares. Por tanto, las claves de este tránsito (Kleiber, 2001) son el desarrollo de la autonomía y la independencia (Deci y Ryan, 2000; Moreno y Martínez, 2006; Aibar et al, 2015), proceso en el que la influencia del entorno familiar tiene un peso específico. La adolescencia es el inicio y la juventud la etapa en la que se continúa trabajando arduamente por el asentamiento de la propia identidad, contextualizada en el desarrollo de una identidad grupal (Kleiber, Hutchinson y Williams, 2002). La persona joven se halla en plena búsqueda del equilibrio, pasada la etapa convulsa de la adolescencia, continua persiguiendo la madurez adulta y está centrado en la importante tarea de orientar su vida y realizar un adecuado proceso de integración de todos los aspectos vitales y de su personalidad, es un momento en el que la persona se encuentra más enfocada hacia la realidad y no tanto a fantasías o deseos más pueriles (Hutchinson, Bland, y Kleiber, 2008).

Durante la etapa vital de la juventud, la socialización es el eje fundamental de desarrollo, y el ámbito del ocio constituye el espacio perfecto para fomentar procesos de socialización al asentar las relaciones interpersonales (Madariaga y Romero, 2016) y cultivando la participación social. Existen diferentes modos 
de entender la participación social, produciéndose diferencias significativas en cuanto al género (Santacreu, 2008) pero a pesar de ello, se puede afirmar que es una de las dimensiones a través de las cuales las personas jóvenes construyen su universo de relaciones grupales y definen imaginarios de la realidad social, en base a un conjunto de expectativas, experiencias pasadas y actitudes presentes (García, 2008).

Es necesario detenerse a continuación en el papel que tienen en el desarrollo juvenil el grupo de pares o iguales, ya que para las personas jóvenes, los otros, constituyen un factor clave para el desarrollo y búsqueda la propia identidad, influyendo en el comportamiento, las actitudes, las conductas, y los pensamientos. El grupo de pares promueve el desarrollo individual y social (Rodríguez, 2010), es un espacio en el que sus componentes son semejantes y se establecen relaciones de igualdad, el líder no es autoritario, se convierte en el principal referente, es el lugar en el que la persona ensaya los procesos de adaptación social. Aunque se dan variaciones en el número y tamaño de los grupos de pares (Rodríguez, Megías, y Sánchez, 2003), todos ellos se constituyen y crecen en base a lazos afectivos y relaciones sociales horizontales, tienen un lenguaje propio y, aunque no están exentos de problemas, se basan en el apoyo mutuo.

En términos generales, son relaciones duraderas y estables, el funcionamiento grupal se basa en pautas estables de conducta con normas y valores compartidos, el grupo constituye el espacio temporal donde la persona aprende a relacionarse con los demás, adquiriendo habilidades, aptitudes y competencias para desenvolverse en sociedad, constituye un relevante espacio de convivencia basado en los afectos y las emociones en el que se desarrolla una amplia disponibilidad y entrega al grupo. Quizá el rasgo más especial es que constituye un grupo elegido (Feixa y Porcio, 2004), en el que la persona joven se vive segura, siente que pertenece a un grupo con el que comparte actividades, intereses, gustos, estilo de vida, y percibe que todos ellos tienen cosas en común (circunstancias, retos, tiempo y cultura).

También cabe constatar la importancia del ocio como ámbito de desarrollo integral a lo largo de la vida (Cuenca, 2014). Este tema ha sido ampliamente estudiado en la población joven (Kleiber, Larson, y Csikszentmihalyi, 2014), diversos estudios avalan que el ocio es un derecho humano básico (San Salvador del Valle, 2000), considerado de tercera generación (Cuenca, 2004; Lázaro, 2006; Herrán, 2007; Madariaga y Lazcano 2014), un ámbito de desarrollo y calidad de vida (Lazcano y Madariaga, 2016a), que contribuye a la promoción de aspectos físicos, cognitivos, emocionales y conductuales tanto en el plano individual como en el social (Hutchinson y Kleiber, 2005). 
A menudo, el enfoque de las investigaciones ligadas al binomio ocio y juventud se centran en problemas asociados a ese grupo de población, siendo los temas más estudiados: el consumo de alcohol (Giménez, Cortés, y Espejo, 2010), drogas, siniestralidad, violencia o riesgos (Comas, 2003), entre otros. Pero en los últimos años, han ido en aumento otro tipo de investigaciones centradas en aspectos constituyentes del ocio, tales como: la disponibilidad de tiempo libre (Salvador, 2009), las actividades de ocio (Lazcano y Madariaga, 2016b) y su frecuencia (INJUVE, 2007; Ricoy, y Fernández, 2016), la caracterización de las prácticas (Madariaga y Lazcano, 2014), las preferencias, intereses y significados del ocio (Lazcano, y Caballo, 2016), o las tipologías de espacios de ocio (Ortega, Lazcano y Baptista, 2015). En todos los estudios aparece reflejada la importancia de salir y estar con el grupo de amigos (Stebbins, 2012), las diferencias de género que también se reflejan en el ámbito del ocio (Videnovi, Peši , y Plut, 2010; Megias y Ballesteros, 2014) y la relevancia del ocio nocturno (Berthet et al. 2016).

A partir de los argumentos teóricos planteados se realiza una recogida de datos de alcance estatal, con instrumentos pertinentes al objeto de estudio, con el objetivo de caracterizar las prácticas de ocio de las personas jóvenes realizadas entre el grupo de pares y si estas inciden en la satisfacción vital.

\section{METODOLOGÍA}

\section{Participantes}

La investigación desarrollada se centró en el estudio de la implicación de las personas jóvenes de 16 a 18 años en la constitución y gestión de los espacios donde desarrollan su ocio, para lo cual se optó por realizar un muestreo probabilístico estratificado (mediante una afijación proporcional) al conjunto de la población de estudiantes de Enseñanza Secundaria Postobligatoria de régimen general de centros públicos, concertados y privados del territorio español (Bachillerato, Formación Profesional de Grado Medio y Formación Profesional Básica). A la hora de realizar las afijaciones proporcionales se optó por tomar como referencia territorial las llamadas Áreas Nielsen, así como el tipo de estudios, la titularidad de los centros educativos y el sexo. La adecuación de la herramienta a la población objeto de estudio, se realizó a través de una aplicación piloto a 140 sujetos distribuidos en ocho comunidades autónomas. Finalmente, con un error muestral del 1,9\%, y un nivel de confianza del 95\%, la muestra obtenida fue de 2694 estudiantes y su distribución porcentual atendiendo a la afijación señalada fue la siguiente (ver Tabla 1). 
Tabla 1. Porcentaje de afijación de la muestra a la distribución de las unidades de muestreo

\begin{tabular}{l|c|c}
\hline Sexo & Mujer & $48,6 \%$ \\
& Hombre & $51,4 \%$ \\
\hline Edad & $16-18$ & $72,8 \%$ \\
& $19-21$ & $25,4 \%$ \\
& 22 o más & $1,6 \%$ \\
\hline Nivel de enseñanza & Bachillerato & $65,0 \%$ \\
& Ciclo formativo & $26,5 \%$ \\
& FPB & $8,5 \%$ \\
\hline Titularidad del centro & Pública & $78,6 \%$ \\
& Privada & $21,4 \%$ \\
\hline
\end{tabular}

\section{Instrumento}

Atendiendo a los objetivos e hipótesis de la investigación se elaboró un cuestionario ad hoc para conocer la organización de los tiempos académicos y de ocio. Este artículo se centra en una de las secciones del cuestionario, la dedicada al tiempo libre, concretamente en las siguientes variables:

Actividad de ocio más importante que practican (variable independiente): los y las jóvenes tenían que indicar la actividad de ocio más importante que realizan de un listado de 49 posibilidades. Estas actividades, para favorecer su identificación, se presentaron agrupadas en 9 modalidades, dicha clasificación ha sido utilizada en multitud de estudios sobre prácticas de ocio, habiendo sido validada en el pretest: Televisión y radio, ocio digital, ocio deportivo, turismo y excursionismo, ocio cultural, ocio festivo, juegos (sin ordenador), otras actividades y ocio solidario.

Satisfacción con las actividades de ocio (variables dependientes): a través de una escala Likert con gradación de 5 puntos (nada, poco, algo, bastante, mucho), el alumnado indicó su nivel de acuerdo con las siguientes afirmaciones: "estoy satisfecho con esta actividad", "me siento más satisfecho, disfruto haciendo esta actividad" y "esta actividad es muy importante en mi vida".

Organización en las actividades de ocio (variables independientes): a través de una escala Likert con gradación de 5 puntos (nada, poco, algo, bastante, mucho), el alumnado indicó su nivel de acuerdo con las siguientes afirmaciones: "tengo responsabilidad en la organización de la actividad", disfrutaría más de la actividad si asumiera más protagonismo en su organización" y "participar en la organización del espacio en el que practico la actividad tiene relación con los beneficios que saco de ella". 


\section{Procedimiento}

Dos investigadores se desplazaron a cada centro educativo para la aplicación de los cuestionarios en el aula. Las personas jóvenes participaron en la investigación de forma voluntaria y anónima, siendo informados de los objetivos de la investigación y con el conocimiento y consentimiento de las familias y de los equipos directivos de los centros.

\section{Análisis de datos}

Respecto al primer objetivo, es decir, con el propósito de identificar las modalidades de ocio más significativas, se realizó un análisis de frecuencias, recurriéndose a la prueba Chi-cuadrado para comprobar la relación entre éstas y el género de las personas jóvenes.

En lo referente al segundo objetivo, relacionado con la constatación de si estas modalidades inciden en la satisfacción y significación vital de las mismas, es decir, su efecto en las variables dependientes, se llevó a cabo un análisis de la varianza (ANOVA), también se calculó la d de Cohen (Faul, Erdfelder, Lang y Buchner, 2007) para conocer el tamaño del efecto.

Por último, con relación al tercer objetivo, para contrastar si las personas jóvenes que participan en mayor medida en la organización de estas experiencias valiosas se encuentra relacionado con una mayor satisfacción, disfrute e importancia de la actividad, se realizó un análisis de correlaciones entre las variables relacionadas con la organización y la satisfacción explicadas, distinguiendo, también, el género de las personas jóvenes. Se valoró la significación de las diferencias de efectos independientes en hombres y mujeres encontrados para cada variable mediante la transformación en $Z_{\mathrm{r}}$ de Fisher y utilizando la aplicación de Preacher (2002).

\section{RESULTADOS}

El análisis de frecuencias mostró que las actividades de ocio más significativas para las personas jóvenes que cursan Enseñanza Secundaria Postobligatoria en España son: ocio deportivo (33,4\%), ocio festivo (13,3\%), ocio cultural $(17,2 \%)$ y ocio digital $(14,1 \%)$ y otras actividades $(12,6 \%)$ entre ellas, estar con los amigos o cuidar animales. Estas cinco modalidades agrupan al 86,6\% de la muestra, el $13,4 \%$ practica otro tipo de modalidades de ocio como turismo y excursionismo u ocio solidario.

En cuanto al segundo objetivo, y como se aprecia en la Figura 1, los y las jóvenes que muestran una mayor satisfacción con la actividad de ocio que practican, son aquellos y aquellas que practican un ocio de carácter solidario 
Figura 1. Satisfacción con la actividad según modalidad

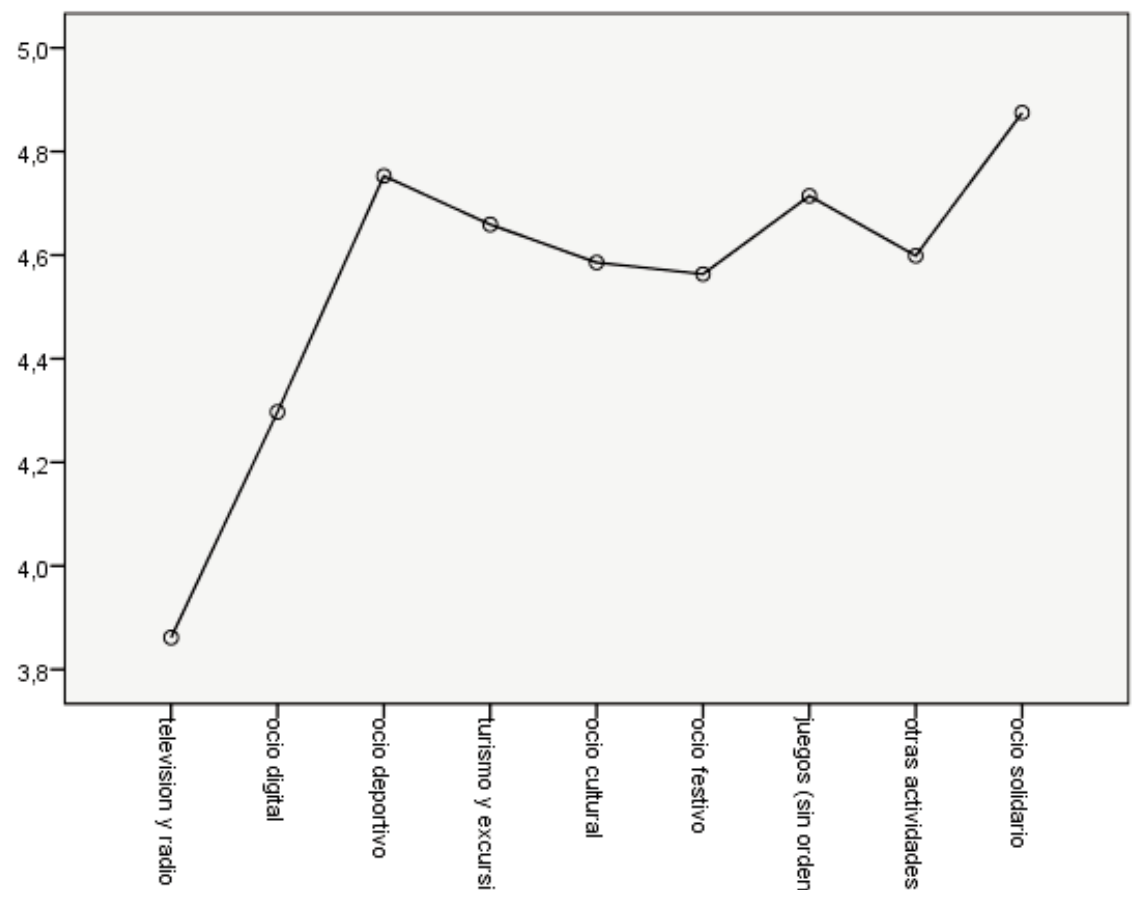

$(M=4,88)$ frente a la modalidad de televisión y radio $(M=3,86)$. Los resultados de la prueba ANOVA indican la existencia de diferencias significativas en la satisfacción de la actividad para las personas jóvenes según la modalidad de ocio que prefieren ( $\mathrm{F}=21,44 ; p \leq .001 ; d=, 26)$.

Como muestra la Figura 2, los y las jóvenes se sienten más satisfechos y disfrutan más realizando un ocio deportivo $(M=4,79)$ y un ocio solidario $(M=4,78)$. La modalidad de ocio con la que menos disfrutan, al igual que en el caso anterior, es con la televisión y la radio $(M=3,79)$. Los resultados de la prueba ANOVA indican la existencia de diferencias significativas en la satisfacción y disfrute de la actividad para las personas jóvenes según la modalidad de ocio que realizan $(\mathrm{F}=28,28 ; p \leq, 001 ; d=, 29)$.

Por último, tal y como se aprecia en la Figura 3, las personas jóvenes cuya principal modalidad de ocio es el asociacionismo y el voluntariado también son los que muestran una mayor significación en relación a dicha modalidad en sus vidas $(M=4,80)$, frente a actividades relacionadas con la televisión y la radio $(\mathrm{M}=2,84)$ que es las que menos significación vital tiene para los y las jóvenes, estando formados estos grupos fundamentalmente por mujeres. En este caso 
Figura 2. Mayor satisfacción y disfrute mientras se realiza según modalidad

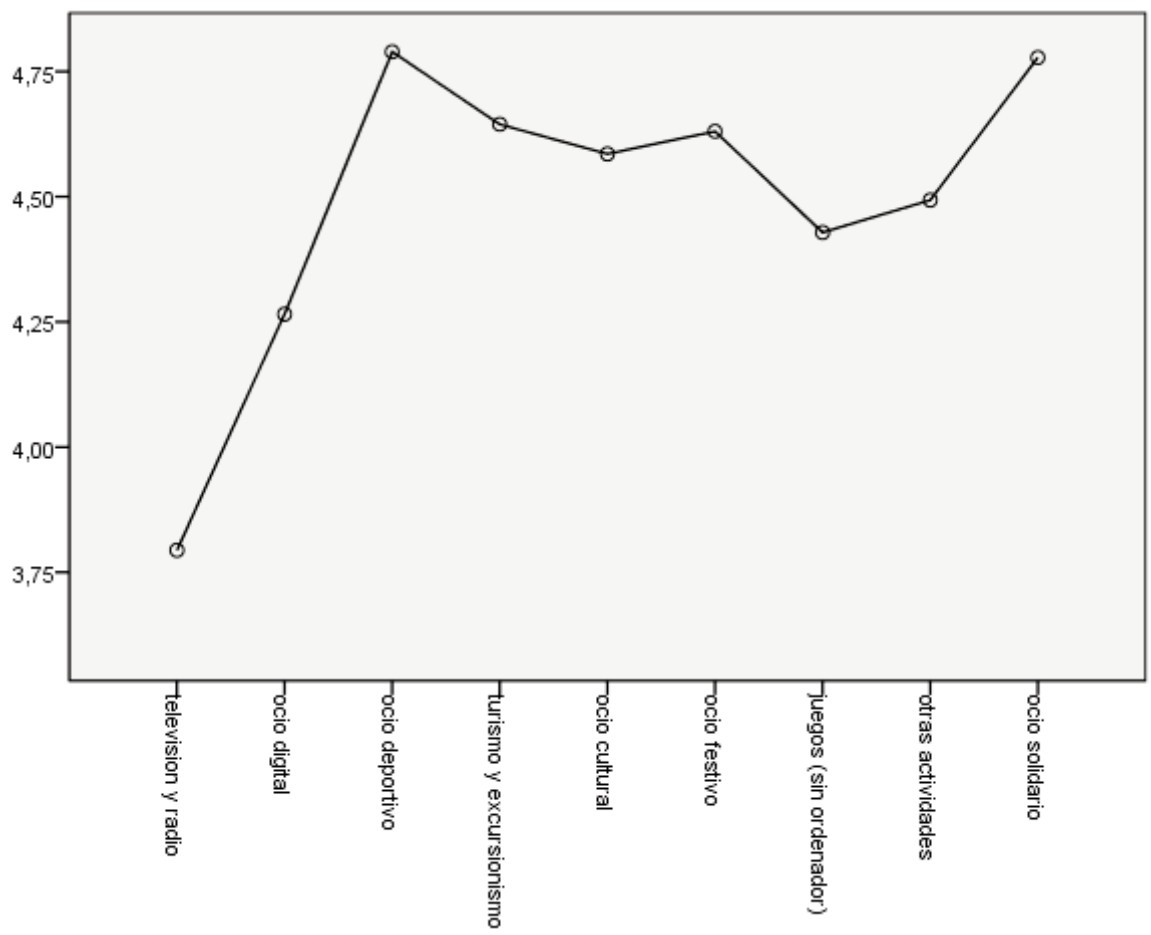

Tabla 2. Relación entre la satisfacción y nivel de organización en ocio Organización

\begin{tabular}{|c|c|c|c|c|c|c|c|c|c|}
\hline & \multicolumn{3}{|c|}{$\begin{array}{l}\text { Tengo responsabilidad } \\
\text { en la organización }\end{array}$} & \multicolumn{3}{|c|}{$\begin{array}{l}\text { Disfrutaria más si } \\
\text { asumiera mayor } \\
\text { protagonismo en } \\
\text { la organización }\end{array}$} & \multicolumn{3}{|c|}{$\begin{array}{l}\text { Participar en la } \\
\text { organización tiene } \\
\text { relación con los } \\
\text { beneficios }\end{array}$} \\
\hline & $\mathrm{M}$ & $\mathrm{H}$ & $p$ & $\mathrm{M}$ & $\mathrm{H}$ & $p$ & M & $\mathrm{H}$ & $p$ \\
\hline $\begin{array}{l}\text { Estoy satisfecho } \\
\text { con la actividad }\end{array}$ & $.23 * *$ & $.22^{* *}$ & .785 & .03 & .01 & .604 & $.16^{* *}$ & $.11^{* *}$ & 187 \\
\hline $\begin{array}{l}\text { Disfruto más } \\
\text { haciéndolo }\end{array}$ & $.24 * *$ & $.24 * *$ & 1.000 & $-.10^{* *}$ & -.02 & .038 & $.14^{* *}$ & $.12^{* *}$ & .598 \\
\hline $\begin{array}{l}\text { Es muy importante } \\
\text { en mi vida }\end{array}$ & $.28^{* *}$ & $.24 * *$ & .266 & ,04 & $.11^{* *}$ & .068 & $.22 * *$ & $.17^{* *}$ & .178 \\
\hline
\end{tabular}

Nota.- ** La correlación es significativa a nivel p<.01. 
Figura 3. Significación vital de la actividad según modalidades

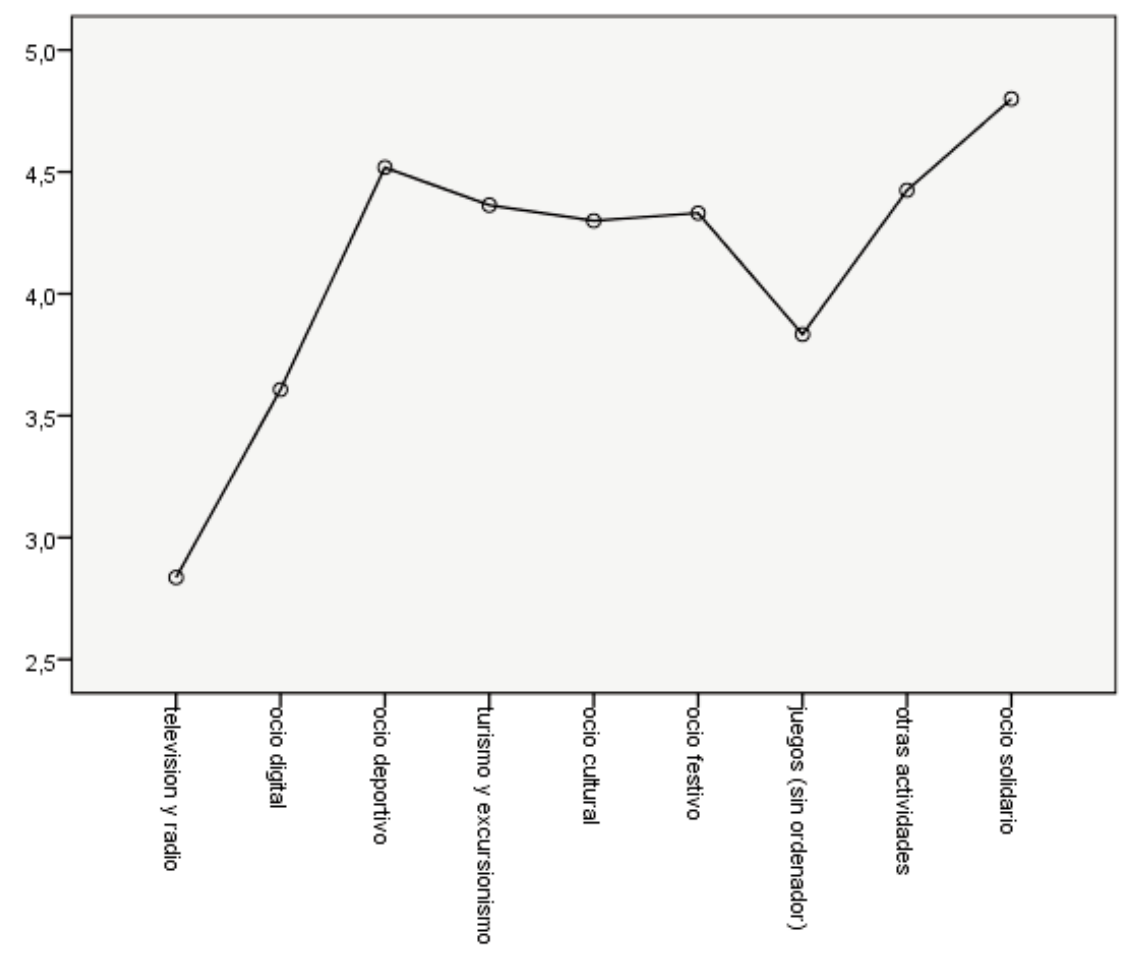

la prueba ANOVA también ha mostrado diferencias en la significación de la actividad $(\mathrm{F}=56,11 ; p \leq, 001 ; d=, 40)$ siendo el tamaño del efecto el más elevado en todos los análisis.

Con relación al tercer objetivo los resultados muestran que el hecho de asumir responsabilidad en la organización de la actividad, les produce una mayor satisfacción, disfrutan más y la actividad resulta más importante en las vidas de las personas jóvenes, tanto para los hombres, como para las mujeres. Los niveles de satisfacción e importancia, otorgada a la práctica de ocio, no se encuentran relacionados con el hecho de asumir más protagonismo en la actividad, ni en la opinión de que participar en la organización pueda proporcionar más beneficios.

\section{DISCUSIÓN Y CONCLUSIONES}

Las conclusiones se centran en aquellas cuestiones que arrojan luz al debate sobre la responsabilidad de las personas jóvenes en la organización de sus acti- 
vidades de ocio y la relación con la satisfacción con la práctica, y la significatividad de estas en sus vidas.

Los principales ámbitos en los que las personas jóvenes desarrollan sus prácticas son el ocio deportivo (actividades físicas en el medio natural, actividades físicas de oposición y de cooperación), y el ocio cultural, que engloba actividades como leer libros, ir a conciertos o ir al teatro. Sin embargo, las actividades que más satisfacen, es decir, con las que más disfrutan, y que son más significativas para las personas jóvenes son las relacionadas con el ocio solidario, las cuales tienen que ver con el asociacionismo y el voluntariado. A pesar de ser una de las modalidades menos practicada por las y los jóvenes, son las que han recibido puntuaciones más altas en las variables analizadas.

Los resultados respecto a la percepción de una mayor satisfacción y disfrute con la práctica realizada han sido prácticamente similares, sin embargo, el tamaño del efecto en esta última variable ha sido superior. Con relación a los niveles de satisfacción en la organización de las actividades, las y los jóvenes se sienten más satisfechos y la actividad supone más para ellos y ellas si participan en su organización, aunque un mayor protagonismo no influye en sus niveles de disfrute y centralidad. En esta línea, señalar que diversos estudios empíricos (Ortega, Lazcano, y Baptista, 2015; Lazcano y Caballo, 2016) han mostrado que las personas jóvenes participan más y con mayores niveles de satisfacción en aquellos espacios de ocio que comparten exclusivamente con su grupo de pares y que además cuentan con niveles elevados de autogestión. Lo que explicaría que los espacios monitorizados en los que su percepción de libertad es mayor, muestren una imagen social de éxito (centrada en la participación), así como que los espacios propios (autogestionados) proliferen entre el colectivo, y perduren durante determinadas fases de este ciclo vital. Así, las personas jóvenes muestran más satisfacción y dotan de mayor significatividad a los espacios y tipos de prácticas en los que adquieren una mayor autonomía y oportunidad de autogestión. Por tanto, los lugares donde los y las jóvenes se sienten más libres son aquellos alejados de la visión de los adultos.

En cuanto a las actividades que tienen más éxito entre las personas jóvenes destacan las que no requieren un compromiso permanente y aquellas que han sido organizadas y creadas previamente por las y los jóvenes, especialmente aquellas que les permite reunirse con su grupo de pares.

Sin embargo no se ha podido demostrar la existencia de un efecto significativo de la modalidad o el ámbito de ocio con la satisfacción con la actividad, pero sí con la significación de esa actividad en el estilo de vida en ocio. Este trabajo no incluye los modos o grados de participación en la organización de las actividades, por lo que no es posible realizar argumentaciones en esta línea. 
La simple implicación en la organización del ocio de las y los jóvenes parece indicar una influencia positiva respecto a la satisfacción con la experiencia y su significación vital, pero es necesario profundizar más en las características de esta participación. Existen postulados conceptuales que refuerzan en cierto modo estos resultados, como es la Teoría de la Autodeterminación (Deci y Ryan, 2000), la cual afirma que cuando las personas participan en una actividad que han elegido y sobre la que tienen control, mejora la motivación intrínseca y su satisfacción. Como señalan Moreno y Martínez (2006) esta teoría constituye un modelo explicativo de la motivación humana que ha sido aplicado a diversos ámbitos, así mismo trabajos aplicados en el ámbito de la actividad física no competitiva han demostrado que una percepción positiva de apoyo de la autonomía se relaciona con niveles más elevados de actividad física y de satisfacción (Aibar et al, 2015).

\section{REFERENCIAS BIBLIOGRÁFICAS}

Aibar, A.; Julián, J.A.; Murillo, B.; García-González, L.; Estrada, S. y Bois, J. (2015). "Actividad física y apoyo de la autonomía: El rol del profesor de Educación Física". Revista de Psicología del Deporte 24 (1), 155-161.

Alaminos, A. (2008). "La juventud embotellada". OBETS. Revista de Ciencias Sociales, 2, $5-23$.

Berthet, M.A.; Lazcano, I.; Lombi, L.; Madariaga, A.; Ramos, A.; Rodríguez, E.; Sanmartín, A. y János, S. (2016). La marcha nocturna: ¿un rito exclusivamente espanol? Madrid: FAD y Centro Reina Sofía pata la Infancia y la Juventud.

Comas, D. (Coord.). (2011). Las políticas públicas de juventud. Madrid: INJUVE

Comas, D. (Dir.). (2003). Jóvenes y estilos de vida. Madrid: INJUVE y FAD.

Csikszentmihalyi, M. y Hunter, J. (2003). "Happiness in everyday life: The uses of experience sampling". Journal of Happiness Studies 4(2), 185-199.

Cuenca, M. (2014). Ocio valioso. Documentos de Estudios de Ocio, núm. 52. Bilbao: Universidad de Deusto.

Deci, E.L. y Ryan, R.M. (2000). "The "what" and "why" of goal pursuits: Human needs and the self-determination of behavior", Psychological Inquiry, 11, 227-268.

Elzo, J. y Megías, E. (Codir.). (2014). Jóvenes y valores I. Un ensayo de tipología. Madrid: FAD, Centro Reina Sofía sobre Adolescencia y Juventud y Fundación ONCE.

Faul, F; Erdfelder, E.; Lang, A.G. y Buchner, A. (2007). A flexible statistical power analysis program for the social, behavioral, and biomedical sciences. Behavior Research Methods, 39, 175-191.

Feixa, C. y Porcio, L. (2004). "Los estudios sobre culturas juveniles en España". Revista Estudios de Juventud, 64, 9-28.

García, FJ. (2008). "El laberinto de la participación juvenil: estrategias de implicación ciudadana en la juventud". OBETS. Revista de Ciencias Sociales, 2, 35-51.

Giménez, J.A., Cortés, M.T. y Espejo, B. (2010). "Consumo de alcohol juvenil: una visión desde diferentes colectivos". Health and Addictions, 10(1), 13-34. 
Gómez-Granell, C. y Julià, A. (2015). Tiempo de crecer, tiempo para crecer. Barcelona: Ajuntament de Barcelona.

Heintzman, P. (2000). "Leisure and Spiritual Well-Being Relationships: A Qualitative Study". Loisir et Société / Society and Leisure, 23(1), 41-69.

Herrán, A.I. (2007). "El ocio como derecho fundamental: perspectiva jurídica del ocio a través de los textos legales internacionales". En E. Aguilar y M.J. Monteagudo (eds.). La experiencia de ocio a debate, más allá del consumo y la participación. Cuadernos de Estudios de Ocio, núm.4. Bilbao: Universidad de Deusto, 57-70.

Hills, P.; Argylea, M. y Reevesb, R. (2000). "Individual differences in leisure satisfactions: An investigation of four theories of leisure motivation". Personality and Individual Differences, 28(4), 763-779.

Hutchinson, S.L. y Kleiber, D.A. (2005). "Gifts of the ordinary: Casual leisure's contributions to health and well-being". World Leisure Journal, 47(3), 2-16.

Hutchinson, S.L.; Bland, A.D. y Kleiber, D.A. (2008). "Leisure and stress-coping: Implications for therapeutic recreation practice". Therapeutic Recreation Journal, 42(1), 9-16.

INJUVE (2007). Encuesta Uso de TIC, Ocio y tiempo libre, Recuperado de http://www. injuve.es/observatorio/ocio-y-tiempo-libre/uso-de-tic-ocio-y-tiempo-libre-infor macion. (Última fecha de consulta, setiembre 2017).

INJUVE (2012). Informe 2012. Juventud en España. Recuperado de http://www.injuve.es/. (Última fecha de consulta, setiembre 2017).

King, K. y Church, A. (2015). Questioning policy, youth participation and lifestyle sports, Leisure Studies, 34:3, 282-302.

Kleiber, D.A. (2001). Leisure experience and human development: A dialectical interpretation. New York: Basic Books.

Kleiber, D.A.; Hutchinson, S.L. y Williams, R. (2002). "Leisure as a resource in transcending negative life events: Self-protection, self-restoration, and personal transformation". Leisure Sciences, 24(2), 219-235.

Kleiber, D.A.; Larson, R. y Csikszentmihalyi, M. (2014). "The experience of leisure in adolescence". En M. Csikszentmihalyi, Applications of flow in human development and education. New York: Springer Science, 467-474.

Larson, R. (2000). "Toward a psychology of positive youth development". American Psychologist, 55(1), 170-183.

Lázaro, Y. (2006). "Derecho al ocio”. En M. Cuenca. (coord.). Aproximación multidisciplinar a los estudios de ocio. Documentos de Estudios de Ocio, núm. 31. Bilbao: Universidad de Deusto, 143-156.

Lazcano, I y Madariaga, A. (2016a). "El valor del ocio en la sociedad actual". En. M.A. Berthet; I. Lazcano; L. Lombi; A. Madariaga; A. Ramos; E. Rodríguez; A. Sanmartín y S. János (2016). La marcha nocturna ¿Un rito exclusivamente español? Madrid: FAD y Centro Reina Sofía sobre Adolescencia y Juventud, 15-33.

Lazcano, I y Madariaga, A. (2016b). "El Ocio Nocturno de la Juventud en España". En. M.A. Berthet; I. Lazcano; L. Lombi; A. Madariaga; A. Ramos; E. Rodríguez; A. 
Sanmartín y S. János (2016). La marcha nocturna ¿Un rito exclusivamente español? Madrid: FAD y Centro Reina Sofía sobre Adolescencia y Juventud, 34-95.

Lazcano, I. y Caballo, B. (2016). "Ocio deportivo juvenil: relación entre satisfacción e implicación organizativa". Revista de Psicología del Deporte, 25(2), 9-14.

Leversen, I.; Danielsen, A.G.; Birkeland, M.S. y Oddrun, S. (2012). "Basic psychological need satisfaction in leisure activities and adolescents' life satisfaction". Journal of Youth and Adolescence. 41(12), 15-23.

Madariaga, A. y Lazcano, I. (2014). Estudio sobre la situación del ocio de la juventud con discapacidad en Bizkaia. Diputación Foral de Bizkaia. Bilbao: Diputación Foral de Bizkaia.

Madariaga, A. y Romero, S. (2016). "Barreras percibidas entre los jóvenes para no participar en actividades de ocio". Revista de Psicología del Deporte, 25(2), 21-26.

Megías, I. y Ballesteros, J. (2014). Jóvenes y género. Estado de la cuestión. Madrid: FAD.

Moreno, J.A. y Martínez, A. (2006). "Importancia de la Teoría de la Autodeterminación en la práctica físico-deportiva: Fundamentos e implicaciones prácticas". Cuadernos de Psicología del Deporte, 6(2), 39-54.

Onishi, J.; Masuda, Y.; Suzuki, Y.; Gotoh, T.: Kawamura, T. y Iguchi, A. (2006). The pleasurable recreational activities among community-dwelling older adults. Archives of Gerontology and Geriatrics, 43(2), 147-155.

Ortega, C.; Lazcano, I. y Baptista, M.M. (2015). "Espacios de ocio para jóvenes, de la monitorización a la autogestión”. Pedagogía Social. Revista Interuniversitaria, 25, 69-89.

Preacher, K.J. (2002). Calculation for the test of the difference between two independent correlation coefficients [Computer software]. Retrieved from: http://quantp sy.org/corrtest/corrtest.htm

Ricoy, M.C. y Fernández, J. (2016). "Prácticas y recursos de ocio en la adolescencia". Educatio Siglo XXI, 34(2), 103-124.

Rodríguez, E; Megías, I. y Sánchez, E. (2003). Jóvenes y relaciones grupales: dinámica relacional para los tiempos de trabajo y de ocio. Madrid: INJUVE - FAD.

Rodríguez, E. (2010). "Socialización y referentes socio-grupales en la construcción de identidad juvenil". Educación y Futuro: Revista de investigación aplicada y experiencias educativas, 22, 55-70.

Sacker, A. y Cable, N. (2006). "Do adolescent leisure-time physical activities foster health and well-being in adulthood? Evidence from two British birth cohorts". The European Journal of Public Health, 16(3), 331-335

Salvador, T. (2009). Adolescentes y jóvenes: ocio y uso del tiempo libre en España. Delegación del gobierno para el Plan Nacional sobre Drogas. Madrid: Ministerio de Sanidad, Servicios Sociales e Igualdad.

San Salvador Del Valle, R. (2000). Políticas de ocio. Cultura, turismo, deporte y recreación. Documentos de Estudios de Ocio, núm. 17. Bilbao: Universidad de Deusto.

Santacreu, O. (2008). "La participación social de los jóvenes españoles". OBETS. Revista de Ciencias Sociales, 2, 25-34. 
Stebbins, R.S. (2012). "Compromiso temporal discrecional: efectos sobre la elección y el estilo de ocio". Arbor, 188(754), 293-300.

Videnovi , M.; Peši , J. y Plut, D. (2010). "Young people's leisure time: gender differences". Psihologija, 43(2), 199-214.

IDURRE LAZCANO es doctora en Ocio. Master en Gestión de Ocio Cultural. Pedagoga. Ha sido subdirectora de investigación de Estudios de ocio. Directora del Máster Universitario en Dirección de Proyectos de Ocio. Miembro del equipo oficial de investigación. Docente de grado y posgrado. Ha publicado artículos y libros en el campo del ocio y la cultura, y asistido a congresos. Su línea investigación se centra en ocio cultural, asociacionismo, gobernanza y juventud. Orcid. Org/0000-0002-6746-0357

AURORA MADARIAGA es doctora en Ocio. Máster en Educación Especial. Experto en Pedagogía Terapéutica. Psicóloga. Investigadora Principal del equipo A "El ocio como factor de desarrollo personal" (Gobierno Vasco, IT984-16). Directora de la Cátedra Ocio y Discapacidad. Docente de grado y posgrado. Ha impartido multitud de cursos y ponencias. Además de artículos es coautora de varios libros sobre inclusión, accesibilidad, ocio y discapacidad. Ha dirigido tesis doctorales. Orcid. Org 0000-0002-8369-2781

DOUGLAS A. KLEIBER es profesor de Counseling y Desarrollo Humano en la rama de Psicología y Gerontología de la Universidad de Georgia. Es un experto de renombre en Psicología Social, ha escrito más de 80 artículos en revistas de impacto y varios libros y capítulos de libro sobre ocio y desarrollo humano y sobre la experiencia de ocio y su relación con periodos de tránsitos vitales. Estuvo becado por Ikerbasque en Estudios de Ocio en 2011. Es un activo miembro de WLO.

DANIEL MURIEL es Doctor en Sociología. Actualmente ocupa un puesto como Investigador Postdoctoral en el Instituto de Estudios de Ocio de la Universidad de Deusto (Bilbao). Ha investigado y publicado ampliamente sobre identidad, cultura, videojuegos, patrimonio cultural o juventud. Es co-autor, entre otros, del libro Video Games as Culture (Routledge). Web:https://danielmuriel. net/

Orcid:http://orcid.org/0000-0002-5651-0750

Recibido: 05/04/2017

Aceptado: 16/09/2017 


\title{
EMPRENDIMIENTO Y OCIO EN LA FORMACIÓN INICIAL DEL PROFESORADO DE EDUCACIÓN PRIMARIA ENTREPRENEURSHIP AND LEISURE IN THE INITIAL TRAINING OF PRIMARY EDUCATION TEACHERS
}

\author{
María Isabel Luis Rico \\ Universidad de Burgos, España \\ miluis@ubu.es \\ Tamara de la Torre Cruz \\ Universidad de Burgos, España \\ tdtorre@ubu.es \\ Alfredo Jiménez Palmero \\ Kedge Business School, Francia \\ alfredo.jimenez@kedgebs.com
}

\section{Cómo citar / Citation}

Luis Rico, María Isabel; de la Torre Cruz, Tamara y Jiménez Palmero, Alfredo (2017) "Emprendimiento y ocio en la formación inicial del profesorado de Educación Primaria". OBETS. Revista de Ciencias Sociales, 12(Extra 1): pppp. 121-149. doi:10.14198/OBETS2017.12.1.15

\section{Resumen}

Nuestra investigación se centra en la educación formal, como garante de la adquisición de la nueva configuración competencial que permite un desarrollo social e individual satisfactorio. Dentro de ella, el docente es el factor intraescolar de mayor impacto en el rendimiento, por lo que su desempeño profesional ha de estar acorde a los retos planteados.

Así, si consideramos la competencia emprendedora y la educación para el ocio como aspectos relevantes en la configuración del modelo de ciudadanía participativa, han de verse reflejados en los procesos de formación inicial del docente, como responsable de su posterior desarrollo en la población. 
Palabras clave: desempeño docente; ocio; emprendimiento; formación inicial. Abstract:

This paper focuses on formal education, as guarantor of the acquisition of the new competence configuration that allows a satisfactory social and individual development. Within it, the teacher is the intra-school factor with the greatest impact on performance, so that his or her professional performance must be in line with the challenges posed. Thus, considering entrepreneurship and leisure education as relevant aspects in shaping the model of participatory citizenship, they must be reflected in the processes of initial teacher training, as responsible for their subsequent development in the population.

Keywords: teaching performance, leisure, entrepreneurship, initial formation.

\section{Extended abstract}

The rapid and constant social changes that are taking place in the last decades demand that social, political, economic and educational institutions, among others, promote actions to adapt to new realities. In this context, new needs emerge from the citizens which need be satisfied by the institutions, enabling them, through the acquisition of new competencies, to exercise an active, participatory and democratic citizenship that helps to achieve sustainable social development.

In this context, education plays a fundamental role in favoring and developing in the individual the basic competencies necessary to successfully develop professionally in a global, multicultural and changing world. Education therefore must promote the necessary changes to adapt to the new demands. Within the competency model of the new citizenship we consider entrepreneurship and leisure education as fundamental transversal aspects in educational practice, considering, on the one hand, leisure as an activity that the person performs in a planned way and that promotes the personal development, and on the other, entrepreneurship as a current need of society to achieve development. Thus, if leisure and entrepreneurship are part of the contemporary social configuration, compulsory formal education must lead to their acquisition by all citizens, thus giving satisfaction to the principles of inclusion and equity (European Commission, 2012; OECD, 2016; Unesco, 2015/ 2016).

All the actors involved in the educational processes, within the formal compulsory education, are decisive when it comes to achieving the educational objectives and, therefore, to ensure that the graduates of the system are citizens with a high competency performance that Individual and social development. However, the teacher acquires a determining value since there is a significant and positive correlation between the quality of teachers and the achievements of the students, being the most important in-school aspect that impacts school performance (Darling-Hammond, Holtzman, Gatlin and Heilig, 2005; Hanushek, Kain and Rivkin, 2004; OCDE, 2005; OCDE, 2010; Rivkin, 2005; Yoon, Duncan, Lee, Scarloss and Shapley, 2007).

The objective of this study is to analyze the presence of entrepreneurship and leisure competencies as critical determinants of professional development 
focused on the coherence and adequacy of initial teacher training to the current educational context. Proposing the following hypotheses: H1: Within the process of transforming the educational paradigm, entrepreneurship and leisure are critical competencies necessary for the exercise of active citizenship. H2: Entrepreneurial and leisure competencies are present in the configuration of the framework of competencies to be reached in the stages of compulsory education. H3: Entrepreneurial and leisure competencies are present in the configuration of the teaching performance within the initial formation, giving coherence to the university training offer and allowing its adaptation to the reality of the educational context.

Our research focuses on a documental analysis of verified degree reports, using a representative sample of public universities where the Primary Education degree is implemented. To do so, a qualitative methodology is used through the analysis of the data with the ATLAS-Ti software version 7.10, establishing categories and dimensions of analysis. The material analyzed corresponds to documents generated by international organizations and institutions such as: OECD and UNESCO; European Council and European Commission, and at the national level, the legislation in force in the educational field, the materials elaborated around the objectives set and the verification reports of the Primary Teaching Degrees of the Spanish public universities. A total of 56 documents were analyzed: 24 from the international level, 13 from the national scope and 19 Master Degree Verifications verified.

The results of the study show that the areas of acquisition of the competencies have to be implemented in environments of flexible and open learning that allow life-long learning, being the educational areas involved both the formal education as the informal or not formal. Humanistic education, however, clearly indicates how aspects relating to the acquisition of competencies must be carried out within the mandatory stages, since quality, inclusive and equitable education is conceived as a right. Thus, formal and compulsory education guarantees universal access to the necessary skills (Hanushek and Kimko, 2000; Power, 2007). If we consider entrepreneurship and leisure as part of the frame of reference, it is in the obligatory stage where the acquisition processes must begin, training people for their subsequent development, as reflected in their inclusion of the educational legislation that regulates contents and objectives of the mandatory stages. At this point we see the reflected $\mathrm{H} 1$ and $\mathrm{H} 2$.

On the other hand, if we consider that formal compulsory education has to be of quality, the decisive endogenous factor that directly and positively influences school quality and student performance is the teacher (DarlingHammond, Holtzman, Gatlin and Heilig, 2005; Hanushek, Kain and Rivkin, 2004; OCDE, 2005, 2007). The improvement of teacher performance through initial and continuing training is the objective that focuses on educational policies, due to their influence in the educational context.

The characteristics of society make the teaching function and the expectations on it to evolve with the challenges that need an answer, and which require not only the acquisition of new skills and knowledge, but also their conti- 
nuous development through an initial quality training and a coherent process of professional development (Montalvo and Gorgels, 2013; Ronfeldt and Reininger, 2012). In our environment, the teaching function relies on three formative pillars: initial training, continuous and ongoing training and practical training. The permanent and practical training is considered within the continuous professional development and is regulated by the educational legislation of national and autonomous scope. However, the initial training is regulated by the state legislation directed to the university field, which enjoys an organizational and management greater level of autonomy.

With regard to the presence of entrepreneurship and leisure within the initial training of teachers at the university level, we have been able to verify how in the legislative and regulatory fields both are part of the configuration of the competencies to be acquired by teachers. Nevertheless, in the verified memories they do not have either homogeneous or constant presence, which takes to us to confirm that $\mathrm{H} 3$ is not corroborated. We therefore believe it is necessary to promote the development of global frames of professional competencies for teachers, coherently defining the different moments of their acquisition, providing these frameworks with sufficient flexibility and adaptability to the current technologically advanced knowledge society, in which quality, innovation and transfer of knowledge are fundamental. The results show us different processes of implementation of competencies at the university level, which invite us to reflect on the adequacy of the initial teacher training plans.

\section{INTRODUCCIÓN}

La sociedad actual se encuentra inmersa en procesos de cambio que determinan la configuración competencial de los ciudadanos, demandando de las instituciones sociales, económicas, políticas y educativas actuaciones que den satisfacción a la nueva realidad, posibilitando y garantizando el ejercicio de la ciudadanía activa de forma plena y satisfactoria, ya que el principal activo de las sociedades son las personas y su capital humano (Avendaño, Paz y Parada, 2016; Bolívar, 2007; Ganuza y Francés, 2015; Mieles y Alvarado, 2012; Molina, 2015; Raffini, 2015).

En este contexto los organismos internacionales como la OCDE, UNESCO y UE, entre otros, señalan a la educación, en su definición más amplia, como factor determinante en la adquisición de competencias, entendidas como el conjunto de conocimientos, habilidades y destrezas, que satisfagan las demandas individuales y sociales, permitiendo el avance social en pro de los objetivos de desarrollo sostenible que señala la UNESCO para el 2030. En este sentido, la educación fundamenta su finalidad en el desarrollo pleno del individuo y, por ende, de la sociedad a través de la adquisición de competencias en todos los ámbitos educativos que se dan dentro de la sociedad: formal, no formal e infor- 
mal. Afirmaciones que ponen de manifiesto la relevancia de determinar qué tipo de competencias son las necesarias para favorecer el desarrollo exitoso de los individuos y que permita a su vez configurar una sociedad más equitativa e inclusiva (Comisión Europea, 2012; OCDE, 2016; Unesco, 2015/ 2016).

No obstante, es en la educación formal y más concretamente en las etapas obligatorias donde el esfuerzo social por lograr la formación de todos y cada uno de sus ciudadanos se ha de encaminar hacia el desarrollo de las competencias fundamentales, consiguiendo así una sociedad más equitativa e inclusiva. Es en la escuela donde la mayoría de personas permanecen durante al menos 9 años, en las etapas de infantil y primaria, adquiriendo los conocimientos, habilidades y competencias básicas, así como también las normas, actitudes y valores fundamentales que les permita desarrollar sus talentos y alcanzar su potencial de desarrollo. A través de una escuela de calidad se establecen las bases de una sociedad abierta, inclusiva, participativa y democrática, formando a las personas en la ciudadanía responsable y crítica (Hanushek y Kimko, 2000; Power, 2007).

Pero ¿Qué competencias ha de desarrollar la educación dentro de las etapas obligatorias? Es precisamente en este punto donde la competencia emprendedora y la educación para el ocio surgen como aspectos transversales que impregnan la práctica educativa. Así, la educación para el emprendimiento está considerada en la actualidad como factor que empodera al individuo, ya que trasciende al ámbito meramente económico y se enlaza con aspectos de desarrollo personal. Dentro de esta perspectiva dicha competencia es entendida como la habilidad del alumnado para trasformar sus ideas en actos aprovechando los recursos disponibles en su entorno, convirtiéndose en el eje central de la educación para el emprendimiento, que mantiene como objetivo dotar al alumnado de las destrezas y habilidades necesarias para desarrollar una mentalidad emprendedora (Agarwal, Audretsch y Sarkar, 2007).

Las habilidades que lleva pareja la competencia emprendedora -la creatividad y el sentido de la iniciativa; la resolución de problemas y el pensamiento crítico; la toma de decisiones y la asunción de riesgos; la adaptabilidad y la perseverancia; la autodisciplina y el sentido de la responsabilidad; el liderazgo y el trabajo en equipo; competencias de planificación y organización; la comprensión del contexto social, económico y cultural; y destrezas lingüísticas y capacidad de persuasión-, exige el desarrollo gradual desde una edad temprana, a través de su inclusión en las etapas formativas regladas, tal y como lo refleja las recomendaciones de la Agenda de Oslo que incide en la importancia de la etapa de primaria (EACEA/Eurydice, 2012; Grilo y Irigoyen, 2006; Krueger, 2015; Lackeus, 2015; O'Connor, 2013; Palmero et al., 2014; Seikkula, Ruskovaara, Ikavalko, Mattila y Rytkola, 2010). 
En el caso del ocio, se sitúa dentro de la configuración del modelo de competencias escolares en los procesos de flexibilización del aprendizaje, que hace evolucionar los tiempos escolares hacia tiempos de aprendizaje individuales. Este avance posibilita que ambos se conjuguen a lo largo del tiempo vital, alcanzado el objetivo del aprendizaje a lo largo de la vida, añadiendo una nueva variable en la figura del tiempo vital, el tiempo del ocio (Cabeza y Amigo, 2013; Cuenca, 2010; 2011). El tiempo vital, escolar y de ocio coinciden en el objetivo a lograr: desarrollar de forma integral a la persona en el ámbito individual y social, ayudando a modular los efectos negativos del conflicto de la conciliación horaria y dotando de flexibilidad y permeabilidad a las necesidades sociales (Bauman, 2007; Caride, 2012; 2014; Faché, 2002; Fernández, 2001; Mulford, 2010). El tiempo escolar y peri-escolar cohabita con el tiempo de ocio dentro de los espacios escolares, es por ello que se hace necesario su atención educativa y su inclusión en los procesos de aprendizaje de forma consciente y activa (Arroba, 2017; Caballo, 2012; Morán, 2012; Morán y Varela, 2011).

Ambos conceptos, aun perteneciendo a ámbitos de estudio tan alejados, encuentra su nexo de unión en la configuración competencial de la ciudadanía activa y democrática, por lo que corresponde a las instancias educativas garantizar el pleno acceso y su desarrollo (Avendaño, Paz y Parada, 2016; Bolívar, 2007; Mieles y Alvarado 2012; Molina, 2015). Todos y cada uno de los actores implicados en los procesos educativos, dentro de la educación formal obligatoria, son determinantes a la hora de alcanzar los objetivos educativos planteados y, por ende, conseguir que los egresados del sistema sean ciudadanos con un alto desempeño competencial que permitan el desarrollo individual y social. No obstante, el profesor adquiere un valor determinante ya que existe una correlación significativa y positiva entre la calidad del profesorado y los logros de los alumnos, siendo el aspecto intraescolar más importante que impacta en el rendimiento escolar (Angrist y Lavy, 2001; Darling-Hammond, Holtzman, Gatlinby Heilig, 2005; Decker, Mayer y Glazerman, 2004; Hanushek, Kain y Rivkin, 2004; OCDE, 2005; OCDE, 2010; Rivkin, 2005; Yoon, Duncan, Lee, Scarloss y Shapley, 2007). De esta forma la profesión docente se vuelve cada vez más exigente, debido a las cambiantes necesidades del entorno social y laboral y la complejidad añadida de adaptar todos ellos al currículo (Glatthorn, Boschee y Whitehead, 2006).

Nuestro interés investigador se centra en el desarrollo del modelo de competencia docente que permita establecer qué conocimientos, capacidades, habilidades, destrezas, valores, actitudes y comportamientos, son necesarios en los docentes para conseguir el reto de educar a sus alumnos conforme a las nuevas exigencias. Es decir, las competencias profesionales necesarias y suficientes para desarrollar las funciones y conseguir los fines educativos que, en este 
sentido, las nuevas conceptualizaciones marcan a nivel internacional y nacional (Akiba, 2007; Cano, 2007; Esteve, 2004; Glatthorn, 2006; Imbernón, 2004; Martín del Pozo, 2013; Pesquero, 2008; Santelices, 2015; Zabalza, 2004). Dentro de los procesos de adquisición de las competencias docentes nos hemos centrado en los procesos de formación inicial, ya que existe una relación positiva entre la formación inicial y la efectividad docente (Ronfeldt y Reininger, 2012; Montalvo y Gorgels, 2013).

En este punto, nuestra investigación se plantea los siguientes interrogantes: ¿Qué tipo de educación subyace ante los cambios sociales en los que estamos inmersos? ¿Existe un nuevo modelo competencial de referencia dentro del sistema educativo? ¿Se considera el emprendimiento y el ocio como competencias fundamentales en la configuración de la ciudadanía del S. XXI? ¿En el ámbito de la educación formal se considera la competencia emprendedora y la de ocio? ¿En qué aspectos? ¿Dentro de la formación inicial del profesorado se consideran el emprendimiento y el ocio como aspectos nucleares del desempeño docente? Para guiar nuestra investigación en la satisfacción de las preguntas planteadas formulamos las siguientes hipótesis de trabajo:

Hp 1: Dentro del proceso transformador del paradigma educativo, se sitúa el emprendimiento y el ocio como competencias necesarias para el ejercicio de ciudadanía activa.

Hp 2: La competencia emprendedora y el ocio están presentes en la configuración del marco de competencias a alcanzar en las etapas de educación obligatoria.

Hp 3: La competencia emprendedora y de ocio están presentes en la configuración del desempeño docente dentro de la formación inicial, dotando de coherencia la oferta universitaria formativa y permitiendo su adecuación a la realidad del contexto educativo.

\section{METODOLOGÍA}

La metodología empleada se basa en un estudio empírico del contexto educativo que, a través del uso de la metodología cualitativa, trata de corroborar las hipótesis planteadas. La metodología de análisis, en coherencia con el problema de investigación, corresponde a la teoría fundamentada (Mella, 2003; Strauss y Corbin, 2002), que tiene como propósito central elaborar una teoría construida a partir de los datos recopilados, analizados de forma sistemática y categorizados con el objeto de hallar las relaciones entre los diversos conceptos desprendidos a partir de los mismos datos, en este caso, los documentos de entidades internacionales, europeas, nacionales y autonómicas, obteniendo así una mejor comprensión de la realidad estudiada. Así la teoría fundamentada posee como característica central la "fundamentación de los conceptos en los datos" 
(Strauss y Corbin, 2002: 14), incorporando adicionalmente y de forma no menos importante el componente de creatividad por parte del investigador, permitiendo a través de ésta efectuar el análisis con una mirada divergente respecto del fenómeno estudiado, sin dejar de lado el rigor científico del método, estableciendo la construcción de la teoría que subyace.

El método contempla una serie de etapas necesarias para el desarrollo del análisis: codificación abierta, codificación axial y codificación selectiva. La codificación abierta es la referida a la generación de categorías a partir de los textos descriptivos, resultantes del proceso analítico en el cual se distinguen los conceptos, sus propiedades y dimensiones. Posteriormente, en un nivel de profundidad mayor se realiza la codificación axial, la cual busca reconstruir las relaciones entre las categorías para dar cuenta de las teorías que subyacen al fenómeno. En esta línea, se emplea el paradigma, comprendido como un esquema organizativo, permitiendo al analista relacionar la estructura y los procesos, comprendiendo así la complejidad de la realidad estudiada (Cáceres, 2003; Strauss y Corbin, 2002). Por tanto, esta perspectiva que guía el análisis de los datos, busca identificar y poner en relación a los componentes del fenómeno y condiciones. Para efectuar la codificación axial es necesaria la codificación abierta, donde se han construido categorías, con sus propiedades y dimensiones.

Para el análisis de los datos se empleó el software ATLAS-Ti versión 7.10.

\section{Material de análisis}

El material corresponde a documentos generados por organizaciones e instituciones internacionales como son: OCDE y UNESCO; Consejo Europeo y Comisiones europeas, y de ámbito nacional, la legislación vigente en el ámbito educativo, el material elaborado en torno a los objetivos planteados y las memorias de verificación de los Grados de Magisterio de Primaria de las Universidades públicas españolas. Los criterios de selección de los documentos son:

- Ámbito de actuación: nivel internacional; europeo; nacional; autonómico.

- Temático: educación; competencias básicas; competencia emprendedora; ocio; formación inicial del profesorado.

- Vigencia: informes de evaluación; legislación vigente y/o consolidada,

- Disponibilidad: memorias de verificación del grado de magisterio de las universidades públicas de España disponibles en las páginas web de las universidades.

Se analizaron 56 documentos primarios (DP) que se presentan en la Figura 11: 24 del ámbito internacional, 13 del ámbito nacional y 19 memorias de grado verificadas. 


\section{Tabla 1. Documentos seleccionados para el análisis y codificación}

\begin{tabular}{|c|c|c|}
\hline ÁMBITO & $\mathrm{DOC}$ & $\begin{array}{ll}\text { REFERENAA } \\
\end{array}$ \\
\hline \multirow{24}{*}{ INTERNACONAL } & & 1 UNESCO, (2015). Education 2030: Incheon Declaration Towards inclusive and equitable quality education and lifelong leaming for all. UNESCO. París \\
\hline & 2 & 2 UNESCO, (2015). Replantear la educación ¿H Hacia un bien común mundial? UNESCO. Paŕs \\
\hline & & UNESCO, (2016). La educación al sevicio de los pueblos y el planeta: Creación de futuros sosteribles para todos. UNESCO. París \\
\hline & & 4 OECD, (2014). TALIS 2013: Estudo Intemacional de la enseñanza y aprendizaje. OECD umw.oecd.og/edu/talis \\
\hline & 5 & 5 OCDE, (2015): Skills Stratay InformedeDiagóśī. OCDE \\
\hline & & $600 \mathrm{OE}$, (2015). From creativity to initiative building entrepreneurial competencies in schools. OECD \\
\hline & & 7 OCDE, (2015). Entrepreneus hip in education: what, why, when, how. OECD \\
\hline & & $\begin{array}{l}\text { EURYDICE, (2004). La profesión docente en Europa: perfil, tendencias y problemática. Comisión Europea. Buselas. Disporible en } \\
\text { htup://wnw.eurydice.org }\end{array}$ \\
\hline & & $\begin{array}{l}9 \text { RECOMENDAGÓN DEL PARLAMENTO EUROPEO Y DEL CONSEJO de } 18 \text { de diciembre de } 2006 \text { scbre las competencias clave para el aprendizaje permanente } \\
(2006 / 962 / \text { CE) }\end{array}$ \\
\hline & 10 & Comisión de las Comunidades europeas, (2007). Documento de Trabajo de los Servicios de la Comisión: ESOUELAS PARA EL SIGLO XXN SEQ(2007)1009 \\
\hline & 11 & $\begin{array}{l}\text { Comisión de las Comuridades europeas, (2007). Comuricación de la comisión al cons ejo europeo y al parlamento europea. Mejorar la calidad de la } \\
\text { formación del profes orado. Buselas, } 0 \mathrm{M}(2007) 392 \text { fina }\end{array}$ \\
\hline & 12 & $\begin{array}{l}\text { Michael Barbery Mona Moushed. Septiembre 2007. “How the Wotd's BestPefforming Schood Systems Come Out On Top”, Mckinsey \& Compary, Social } \\
\text { Sector Office. }\end{array}$ \\
\hline & 13 & $\begin{array}{l}\text { Consejo de la UE (2009). Conclusiones del Consejo de } 26 \text { de noviem bre de } 2009 \text { sobre el des andllo profesional de los profes ores y directores de centros } \\
\text { docentes. (2009/C } 302 / 04)\end{array}$ \\
\hline & 14 & $\begin{array}{l}\text { Comisión Europea, (2012). Comunicación de la comisión al parlamento europea. Un nuevo concepto de educación: irvertiren las com petencias para } \\
\text { lograr mejares resultados socioeconómicos. SWD (2012) } 371 \text { final }\end{array}$ \\
\hline & 15 & $\begin{array}{l}\text { EURYDICE (2012). El desardollo de las competencias clave en el contexto es colar en Europa: des afios y oportunichdes para la política en la materia. } \\
\text { Comisión Europea. Buselas }\end{array}$ \\
\hline & 16 & $\begin{array}{l}\text { Consejo de la UE (2012). Conclusiones del Consejo de } 26 \text { de noviem bre de 2012, sobre la educación u la formación en Europa 2020- La contribución de la } \\
\text { educacióny la formación a la recuperación económica, al crecimiento y al empleo }\end{array}$ \\
\hline & 17 & EURYDICE (2012). Entrepreneurship Education ats school in Europe. National Strategies, Curicula and Leaming Outcomes. Comisión Europea. Busselas \\
\hline & 18 & Comisión Europea, (2013). Cómo crear mentalidades y capacidades Emprendedoras en la UE. Unión Europea. Buselas \\
\hline & 19 & Consejo de la UE (2014). Conclusiones del Consejo de 20 de mayo de 2014, sobre formación eficaz de los docentes (2014/C $183 / 05)$ \\
\hline & 20 & Consejo de la UE (2015). Conclusiones del Consejo sobre el emprendimiento en la educacióny la formación (2015/C17/02) \\
\hline & 21 & $\begin{array}{l}\text { Comisión Europea, (2015). Entrepreneurs hip Education: a road to success. A compilation of evidence on the impact of entrepreneuship education } \\
\text { stratetegies and measures }\end{array}$ \\
\hline & 22 & Parlamento Europeo (2015). Sobre el fomento del emprendimientojuveril a través de la educacióny la formación (2015/ 2006 (INI)) \\
\hline & 23 & Comisión Europea, (2015). Teaching practices in Primary and Secondary schools in Europe: Insights from Large- Scale Assessments in Education \\
\hline & 24 & EURYDICE (2016). Entrepreneurship Education at school in Europe. Comisión Europea. Buselas \\
\hline \multirow{13}{*}{ NAGONAL } & 25 & ANECA, (2004). Informe de la Comisión Libro Blanco del títuo de Grado en Magisterio Vol. Iy Vol. II \\
\hline & 26 & $\begin{array}{l}\text { ORDEN EQ/3857/2007, de } 27 \text { de diciembre, por la que se establecen los requisitos para la verificación de los títulos universitarios oficiales que habiliten } \\
\text { para el ejerciciode la profesión de Maestroen Educación Primania. }\end{array}$ \\
\hline & 27 & Pérez Díaz, V. (dir). El prestigio de la profesión docente en España: Percepción y realicad Fundación Botín \\
\hline & 28 & Calleja, T. et al. (2012). Educación en España-Motivos para la esperanza. McKinsey \& Company \\
\hline & 29 & $\begin{array}{l}\text { Miristerios de Educación, Cultura y Depote, (2012) XXI Encuentuo de consejos escolares autonómicos y del estado. El profes orado del S. XW. MEC. } \\
\text { Madid }\end{array}$ \\
\hline & 30 & Ley Orgánica 8/2013, de 9 de díciem bre, para la mejora de la calidad educativa. \\
\hline & 31 & Pellicer, et al., (2013). A prender a emprender. Cómo educarel talento emprendedor. Functación Príncipe de Girona. Grupo Planeta de Cantabria. \\
\hline & 32 & Real Decreto 126/2014, de 28 de febrero, por el que se es tablece el curículo básico de la Educación Primaria \\
\hline & 33 & Femández-Laviada, (dir.) (2015). Global Enteepreneurship Monitor. Informe GEM España 2015.Universidad \\
\hline & 34 & $\begin{array}{l}\text { Orden ECD/65/2015, de } 21 \text { de enero, por la que se describen las relaciones entbe las com petencias, los conteridos y los criterios de evaluación de la } \\
\text { educación pimaria, la educación secundaria obligatoria y el bachillerato. }\end{array}$ \\
\hline & 35 & Ministerio de Educación, cultura y depate, (2015). La educación para el emprendimiento en el sistema educativo español. REDIE. Madid \\
\hline & 36 & Marina, J.A. (2015). Libro blanco de la profesión docente y su entomo escolar \\
\hline & 37 & Ministerio de Educación, Cultura y Depote, (2016). Panorama de la Educación Indicadores de la OCDE 2016: Informe Español. MEC Madid \\
\hline \multirow{19}{*}{$\begin{array}{l}\text { MEMORIASDE } \\
\text { VERFICACÓN }\end{array}$} & 38 & Memoria de verificación de la Universichad de Alicante \\
\hline & 39 & Memoria de verificación de la Universicad Almería \\
\hline & 40 & Memoria de verificación de la Universichad Burgos \\
\hline & 41 & Memoria de verificación de la Universichad Cantabria \\
\hline & 42 & Memoria de verificación de la Universicdad Castilla la Mancha \\
\hline & 43 & Memoria de verificación de la Universicad Extremadura \\
\hline & 44 & Memoria de verificación de la Universichad Girona \\
\hline & 45 & Memoria de verificación de la Universichad Granada \\
\hline & 46 & Memoria de verificación de la Universicad Huelva \\
\hline & 47 & Memoria de verificación de la Universictad Islas Baleares \\
\hline & 48 & Memoria de verificación de la Universidad Jaén \\
\hline & 49 & Memoria de verificación de la Universichad La Rioja \\
\hline & 50 & Memoria de verificación de la Universichad Las Palmas de GC \\
\hline & 51 & Memoria de verificación de la Universidad Málaga \\
\hline & 52 & Memoria de verificación de la Universicdad País Vasco \\
\hline & 53 & Memaria de verificación de la Universicad Navara \\
\hline & 54 & Memoria de verificación de la Universidad Valencia \\
\hline & 55 & Memoria de verificación de la Universidad Valladolid \\
\hline & 56 & Memoria de verificación de la Universidad Zaragoza \\
\hline
\end{tabular}

Elaboración propia 


\section{DISCUSIÓN DE RESULTADOS}

A partir del contenido de los documentos seleccionados y en relación a las hipótesis de investigación planteadas, se han identificado diferentes categorías a través de la codificación abierta, realizada en base a un proceso iterativo entre la conceptualización de los datos y la elaboración de categorías con sus dimensiones, reconociendo las relaciones primarias entre dichos conceptos. En esta línea de análisis, las principales categorías identificadas con sus dimensiones son las que se muestran en la Tabla 2:

Tabla 2. Categorías y dimensiones de análisis

\begin{tabular}{|c|c|c|}
\hline CATEGORÍAS & CATEGORÍAS EMERGENTES & DIMENSIONES \\
\hline \multirow{11}{*}{$\begin{array}{l}\text { SOCIEDAD } \\
\text { ACTUAL }\end{array}$} & \multirow{11}{*}{$\begin{array}{l}\text { Aspectos que caracterizan } \\
\text { la sociedad actual }\end{array}$} & Resiliencia \\
\hline & & Diversidad cultural \\
\hline & & NNTT \\
\hline & & Innovación \\
\hline & & Avances Científicos \\
\hline & & Cambio social \\
\hline & & ODS \\
\hline & & Nueva ciudadanía \\
\hline & & Empoderamiento \\
\hline & & Conocimiento \\
\hline & & Creatividad \\
\hline \multirow[t]{12}{*}{ EDUCACIÓN } & \multirow[t]{5}{*}{ Conceptos de Educación } & Como derecho \\
\hline & & De calidad \\
\hline & & Equitativa \\
\hline & & Humanista \\
\hline & & Inclusiva \\
\hline & \multirow[t]{7}{*}{ Elementos de la Educación } & Objetivo de la Educación \\
\hline & & Contenido de la Educación \\
\hline & & Innovaciones Pedagógicas \\
\hline & & Trabajo en el aula \\
\hline & & Currículo \\
\hline & & Políticas educativas \\
\hline & & Autonomía de los centros \\
\hline
\end{tabular}




\begin{tabular}{|c|c|c|}
\hline CATEGORÍAS & CATEGORÍAS EMERGENTES & DIMENSIONES \\
\hline \multirow[t]{7}{*}{ APRENDIZAJE } & \multirow[t]{4}{*}{ Conceptos de Aprendizaje } & Aprendizaje abierto \\
\hline & & Aprendizaje flexible \\
\hline & & Aprendizaje móvil \\
\hline & & Aprendizaje permanente \\
\hline & \multirow[t]{3}{*}{ Elementos del aprendizaje } & Resultados de aprendizaje \\
\hline & & Redes de aprendizaje \\
\hline & & Rendimiento \\
\hline \multirow[t]{24}{*}{ COMPETENCIAS } & \multirow[t]{8}{*}{ Competencias clave } & Aprender a aprender \\
\hline & & $\begin{array}{c}\text { Comunicación en lengua } \\
\text { extranjera }\end{array}$ \\
\hline & & Comunicación en lengua materna \\
\hline & & Conciencia y expresión cultural \\
\hline & & Digital \\
\hline & & Iniciativa y espíritu de empresa \\
\hline & & Matemática y CyT \\
\hline & & Sociales y cívicas \\
\hline & \multirow{6}{*}{$\begin{array}{l}\text { Competencias cognitivas y } \\
\text { competencias básicas }\end{array}$} & Aritmética \\
\hline & & Escritura \\
\hline & & Lectura \\
\hline & & Alfabetización digital \\
\hline & & Comunicación \\
\hline & & TIC \\
\hline & \multirow[t]{7}{*}{ Competencias no cognitivas } & Autocontrol \\
\hline & & Colaboración \\
\hline & & Competencias emocionales \\
\hline & & Competencias sociales \\
\hline & & Creatividad \\
\hline & & Pensamiento crítico \\
\hline & & Perseverancia \\
\hline & \multirow{3}{*}{$\begin{array}{c}\text { Competencia Transferibles } \\
\text { y Transversales }\end{array}$} & Educación Cívica \\
\hline & & Resolución de problemas \\
\hline & & Trabajo en equipo \\
\hline
\end{tabular}




\begin{tabular}{|c|c|c|}
\hline CATEGORÍAS & CATEGORÍAS EMERGENTES & DIMENSIONES \\
\hline & Competencia emprendedora & $\begin{array}{c}\text { Indicadores de la COMP } \\
\text { emprendedora }\end{array}$ \\
\hline & $\begin{array}{c}\text { Competencia de mayor } \\
\text { nivel o del S. XXI }\end{array}$ & $\begin{array}{c}\text { Competencias de mayor } \\
\text { complejidad y nivel }\end{array}$ \\
\hline \multirow[t]{10}{*}{ DOCENTE } & \multirow[t]{2}{*}{ Competencia docente } & Competencia docente avanzadas \\
\hline & & Competencia docente básica \\
\hline & \multirow[t]{2}{*}{ Función docente } & Docente como factor clave \\
\hline & & Docente como guía \\
\hline & Desempeño docente & Desempeño docente \\
\hline & \multirow[t]{2}{*}{ Dificultades docentes } & $\begin{array}{l}\text { Desprofesionalización } \\
\text { de los docentes }\end{array}$ \\
\hline & & $\begin{array}{l}\text { Dificultades de la formación } \\
\text { permanente e inicial del } \\
\text { profesorado }\end{array}$ \\
\hline & \multirow[t]{3}{*}{ Formación docente } & Formación inicial docente \\
\hline & & $\begin{array}{l}\text { Formación permanente } \\
\text { docente o Desarrollo } \\
\text { profesional continuo }\end{array}$ \\
\hline & & Universidad y formación docente \\
\hline \multirow[t]{2}{*}{$\begin{array}{l}\text { EMPREN- } \\
\text { DIMIENTO }\end{array}$} & \multirow[t]{2}{*}{ Elementos del emprendimiento } & $\begin{array}{c}\text { Ecosistema de la educación } \\
\text { emprendedora }\end{array}$ \\
\hline & & Intraemprendimiento \\
\hline $\mathrm{OCIO}$ & Ocio & Ocio \\
\hline
\end{tabular}

Elaboración propia

En la Figura 1 situamos la distribución de las citas de los documentos primarios a través de las familias establecidas tanto para los DP como para los códigos y categorías establecidas, no obstante, no hemos referido las citas encontradas en las memorias de grado, ya que se analizan con posterioridad. Como podemos observar la mayor concentración de citas se enmarcan dentro de las familias de categorías referidas a los docentes y en los DP de la UE, resaltando la importancia del docente en los textos seleccionados relacionados con la educación.

En el análisis de las relaciones establecidas entre las diferentes dimensiones de las categorías y subcategorías, se ha establecido la vinculación de los nodos, proporcionando densidad a cada uno de ellos como parte de la codifi- 
Figura 1. Número de citas por familias de códigos y de DP

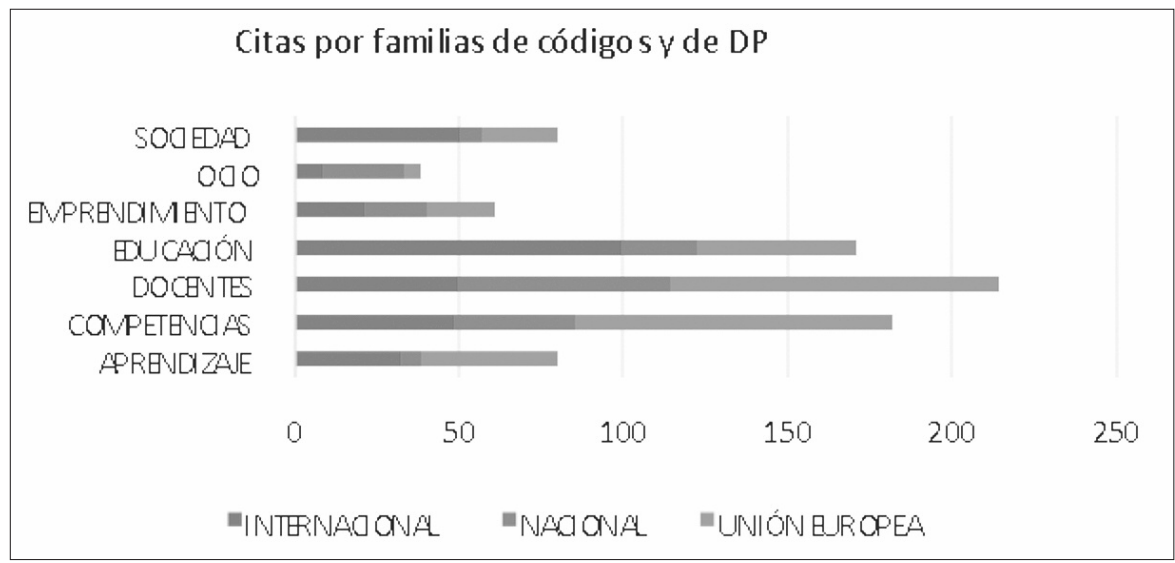

Elaboración propia

cación axial. En la Figura 2 se pueden observar las relaciones primarias entre las dimensiones.

Tal y como se observa, la nueva ciudadanía se construye en función de las características propias de la sociedad actual como son: los procesos de cambio en el ámbito de las nuevas tecnologías provenientes de la innovación, la diversidad cultural, los avances científicos y del conocimiento, entre otros, teniendo como objetivo el desarrollo social sostenible. Esta configuración influye en la nueva conceptualización de los procesos educativos dentro del paradigma de la educación humanista, centrada en la perspectiva de la educación como derecho fundamental que ha de ser equitativa, inclusiva y de calidad. Así, el nuevo marco de competencias debe de estar acorde a las nuevas necesidades para satisfacer los objetivos y fines marcados bajo esta tendencia y ha de ser extensible a todos los ciudadanos, siendo la educación formal obligatoria donde mayor cumplimiento ha de tener, ya que es el medio para capacitar a todas y cada una de las personas en igualdad de condiciones.

Estas competencias se han ido clasificando en función de los procesos y momentos en los que son adquiridas (Figura 3), de tal forma que las competencias básicas o no cognitivas: lectura, escritura, comunicación, aritmética, TIC y alfabetización digital, son la base sobre las que edificar las competencias de mayor nivel o también llamadas del siglo XXI como son las Competencias Clave -Comunicación en lengua extranjera; Comunicación en lengua materna; Digital; Matemática y Científica; Sociales y cívicas; Conciencia y expresión cultural; aprender a aprender e Iniciativa y espíritu emprendedor- y las Competen- 
Figura 2. Configuración de la nueva ciudadanía y educación

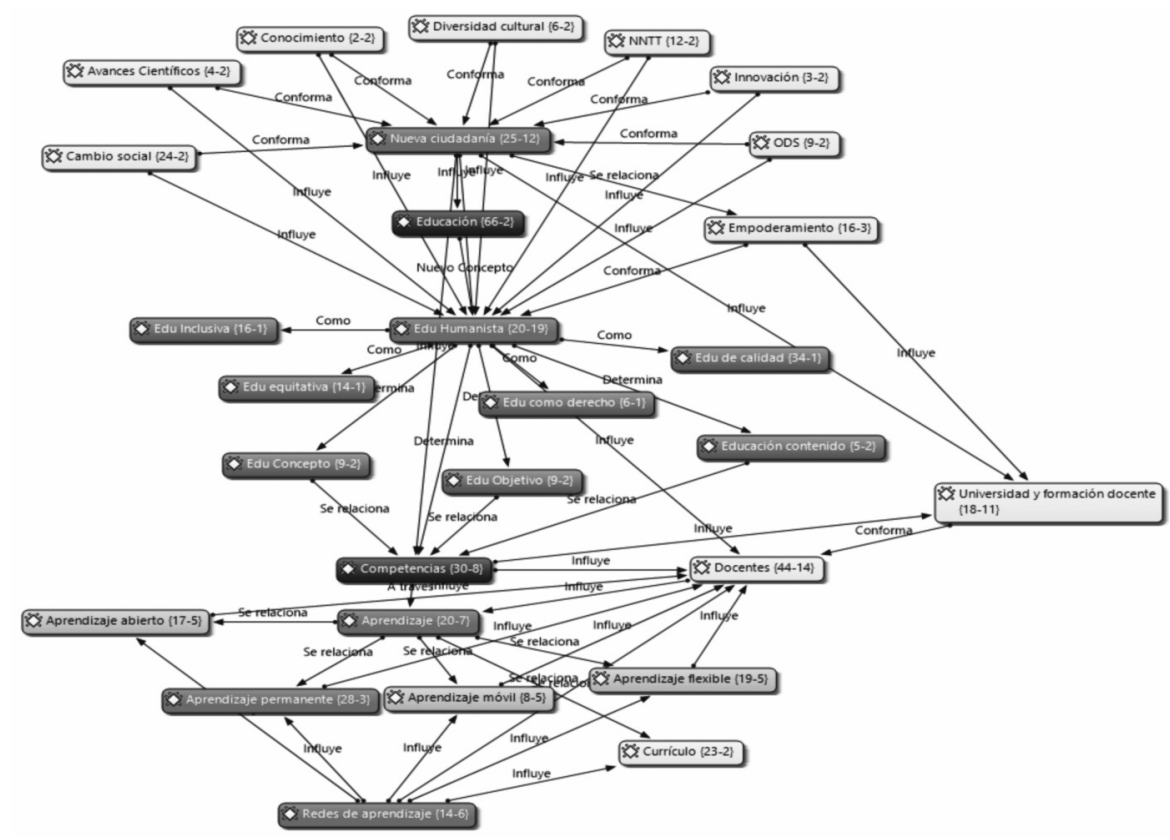

Elaboración propia

cias No Cognitivas -Competencias sociales; Pensamiento crítico; Perseverancia; Autocontrol; Creatividad; Competencias emocionales y Colaboración-, de forma simultánea y con la característica de ser transferibles y transversales encontramos las competencias de Educación cívica, Resolución de problemas y Trabajo en Equipo.

Dentro de este mapa de competencias necesarias para satisfacer las necesidades de la configuración de la nueva ciudadanía, encontramos como la competencia emprendedora y el ocio se vinculan de forma directa con las definiciones de competencias clave como son: la digital; Comunicación en lengua materna; Comunicación en lengua extranjera y Conciencia y expresión cultural, en el caso del ocio, y en su conceptualización en el caso de la competencia emprendedora con la competencia clave de Iniciativa y Espíritu de empresa. Además, se relacionan con competencias No cognitivas, así como con las transversales o transferibles. Así, forman parte de forma directa e indirecta de las competencias de mayor nivel necesarias para el desempeño en el siglo XXI.

Por otro lado, la necesidad de introducir el ocio como aspecto educativo se justifica por la flexibilización del aprendizaje en su ordenación tanto hori- 
Figura 3. Marco competencial de referencia en el nuevo paradigma educativo

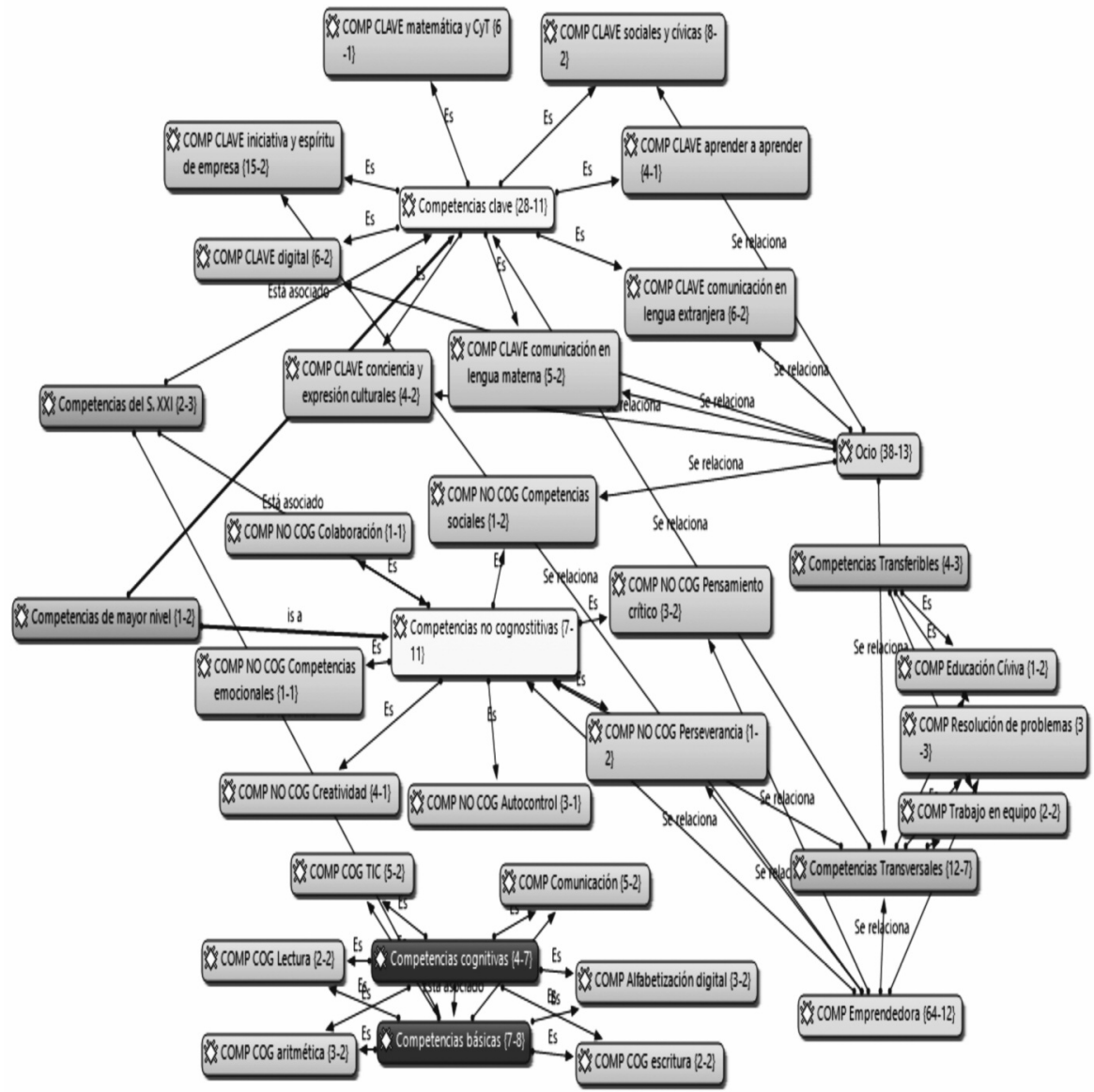

Elaboración propia

zontal, a lo largo de la vida, como vertical, en los tiempos y momentos en los que se organiza los procesos educativos en un mismo día, avanzando hacia momentos y tiempos de aprendizaje móvil y abierto, formando parte de los mismos los tiempos de ocio.

Si consideramos la educación formal obligatoria como el medio por el que capacitar a los ciudadanos a través de las competencias que les permita alcanzar el empoderamiento individual y social, encontramos al docente como el facilitador y factor clave (Figura 4). De esta forma, la calidad del desempeño 
docente se vincula con el éxito educativo, por lo que las competencias docentes, tanto básicas como avanzadas, que han de alcanzar en su formación inicial y continua se relacionan con las establecidas en el marco del nuevo paradigma educativo (Figura 3), del que forma parte tanto la competencia emprendedora y el ocio como ámbito de aprendizaje. Las instituciones responsables de formar a los docentes, en este caso las universidades, se ven requeridas a involucrarse en este cambio fomentando la adquisición de sendas competencias.

\section{Figura 4. La figura docente y su formación}

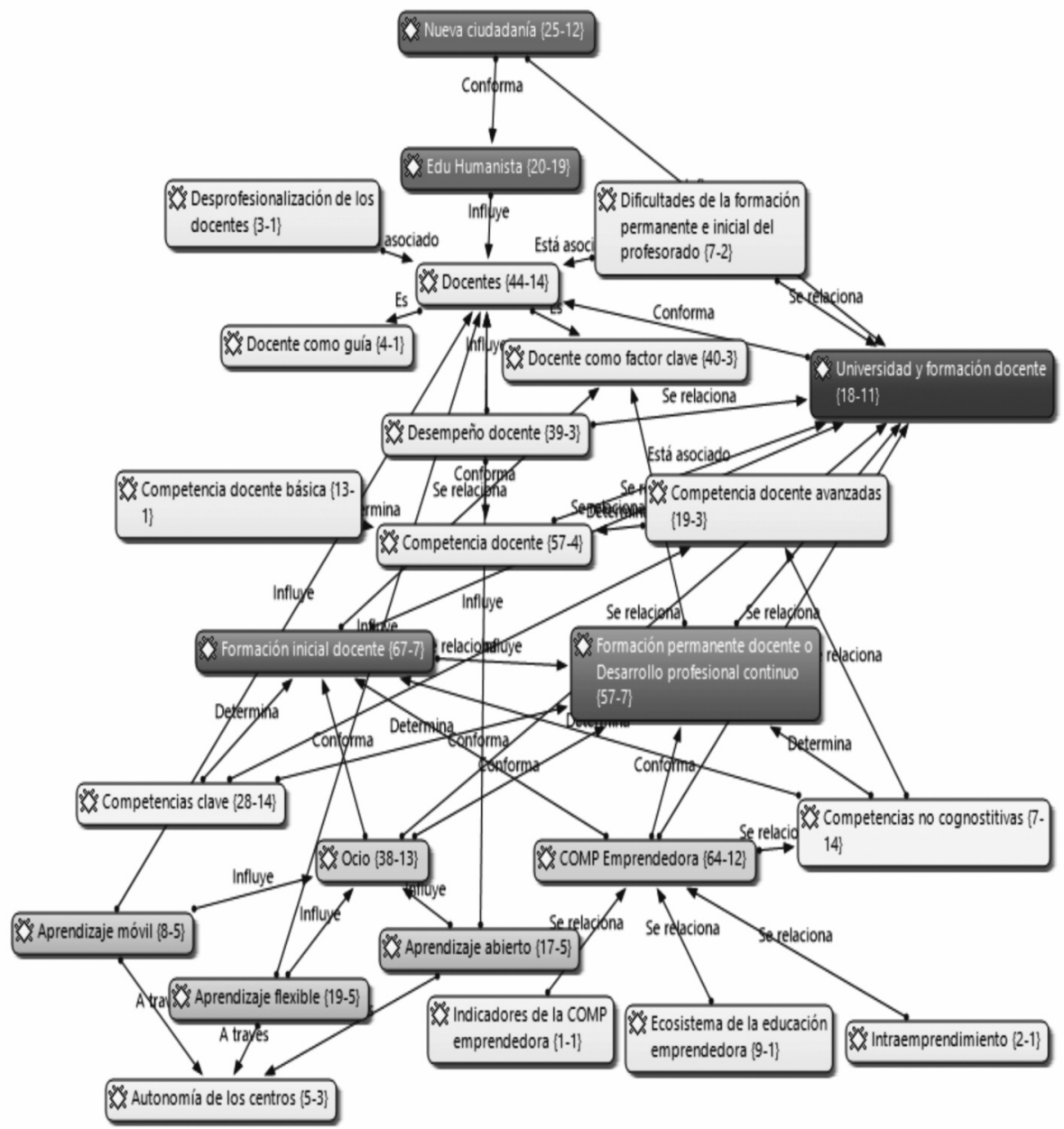

Elaboración propia 
Esta realidad se ve refrendada en los documentos legislativos que rigen la educación formal obligatoria en el ámbito nacional, en la que aluden de forma directa a la necesidad de desarrollar la competencia emprendedora en las etapas obligatorias. Así la LOMCE recoge en el Preámbulo y en su articulado la necesidad de estimular el espíritu emprendedor como fórmula de crecimiento individual y social (Tabla 3). Por otro lado, en su artículo 111 hace referencia a la necesidad de incluir entornos de aprendizajes flexibles y abiertos en los centros, lo que relaciona con la configuración del ocio como entorno de aprendizaje (Tabla 4).

\section{Tabla 3. Referencia sobre la competencia emprendedora en la LOMCE}

\begin{tabular}{|c|c|}
\hline \multirow{7}{*}{$\begin{array}{c}\text { Ley Orgánica } 8 / 2013 \text {, de } 9 \text { de } \\
\text { diciembre, para la mejora de } \\
\text { la calidad } \\
\text { educativa.REFERENCIA } \\
\text { SOBRE EMPRENDIMIENTO }\end{array}$} & $\begin{array}{l}\text { Sin perjuicio de su tratamiento específico en algunas de las áreas de la etapa, la comprensión lectora, la expresión oral y es crita, la comunicación } \\
\text { audiovisual, las Tecnologías de la Información y la Comunicación, el emprendimiento y la educación civica y constitucional se trabajarán en } \\
\text { todas las áreas.») }\end{array}$ \\
\hline & $\begin{array}{l}\text { PREÁMBULO I Todos los estudiantes poseen talento, pero la naturaleza de este talento difiere entre ellos. En consecuencia, el sistema } \\
\text { educativo debe contar con los mecanismos necesarios para reconocerlo y potenciarlo. E reconocimiento de esta diversidad entre alumno o } \\
\text { alumna en sus habilidades y expectativas es el primer paso hacia el desarrollo de una estructura educativa que contemple diferentes trayectorias. } \\
\text { La lógica de esta reforma se basa en la evolución hacia un sistema capaz de encauzar a los estudiantes hacia las trayectorias más adecuadas a sus } \\
\text { capacidades, de forma que puedan hacer realidad sus aspiraciones y se conviertan en rutas que faciliten la empleabilidad y estimulen el espíritu } \\
\text { emprendedor a través de la posibilidad, para el alumnado y sus padres, madres o tutores legales, de elegir las mejores opciones de desarrollo } \\
\text { personal y profesional. Los estudiantes con problemas de rendimiento deben contar con programas especificos que mejoren sus posibilidades de } \\
\text { continuar en el sistema. }\end{array}$ \\
\hline & $\begin{array}{l}\text { IV La educación es la clave de esta transformación mediante la formación de personas activas con autoconfianza, curiosas, emprendedoras e } \\
\text { innovadoras, deseosas de participar en la sociedad a la que pertenecen, de crear valor individual y colectivo, capaces de asumir como propio el } \\
\text { valor del equilibrio entre el esfuerzo y la recompensa. El sistema educativo debe posibilitar tanto el aprendizaje de cosas distintas como la } \\
\text { enseñanza de manera diferente, para poder satisfacer a unos alumnos y alumnas, que han ido cambiando con la sociedad. }\end{array}$ \\
\hline & $\begin{array}{l}\text { VI La reforma promovida por la LOMCE se apoya en evidencias y recoge las mejores prácticas comparadas. Los principales objetivos que } \\
\text { persigue la reforma son reducir la tasa de abandono temprano de la educación, mejorar los resultados educativos de acuerdo con criterios } \\
\text { internacionales, tanto en la tasa comparativa de alumnos y alumnas excelentes, como en la de titulados en Educación Secundaria Obligatoria, } \\
\text { mejorar la empleabilidad, y estimular el es píritu emprendedor de los estudiantes. Los principios sobre los cuales pivota la reforma son, } \\
\text { fundamentalmente, el aumento de la autonomía de centros, el refuerzo de la capacidad de gestión de la dirección de los centros, las evaluaciones } \\
\text { externas de fin de etapa, la racionalización de la oferta educativa y la flexibilización de las trayectorias. }\end{array}$ \\
\hline & $\begin{array}{l}\text { (cb) Desarrollar hábitos de trabajo individual y de equipo, de esfuerzo y de responsabilidad en el estudio, asi como actitudes de confianza en sí } \\
\text { mismo, sentido crítico, iniciativa personal, curiosidad, interés y creatividad en el aprendizaje, y espíritu emprendedor. }\end{array}$ \\
\hline & $\begin{array}{l}\text { Artículo 24. Organización del primer ciclo de Educación Secundaria Obligatoria. En función de la regulación y de la programación de la oferta } \\
\text { educativa que establezca cada Administración educativa y, en su caso, de la oferta de los centros docentes, un mínimo de una y, máximo de } \\
\text { cuatro, de las siguientes materias del bloque de asignaturas específicas, que podrán ser diferentes en cada uno de los cursos: } 33^{\circ} \text { Iniciación a la } \\
\text { Actividad Emprendedora y Empresarial. }\end{array}$ \\
\hline & $\begin{array}{l}\text { La Formación Profesional en el sistema educativo contribuirá a que el alumnado consiga los resultados de aprendizaje que le permitan: Afianzar } \\
\text { el espíritu emprendedor para el desempeño de actividades e iniciativas empresariales }\end{array}$ \\
\hline
\end{tabular}

Elaboración propia

Tabla 4. Referencia sobre el ocio en la LOMCE

\begin{tabular}{|c|c|}
\hline $\begin{array}{l}\text { Ley Orgánica 82013, de } 9 \text { de } \\
\text { diciembre, para la mejora de la } \\
\text { calidad educativa.REFERENCIA } \\
\text { SOBREOCIO }\end{array}$ & $\begin{array}{l}\text { ARTíCULO } 111 \text { Los entormos virtuales de aprendizaje que se empleen en los centros docentes sostenidos con fondos públicos facilitarán la aplicación de planes } \\
\text { educativos especificos diseñados por los docentes para la consecución de objetivos concretos del curriculo, y deberán contribuir a la extensíón del concepto de aula en } \\
\text { el tiempo y en el espacio. Por ello deberán, respetando los estándares de interoperabilidad, pemitiri a los alumnos y alumnas el acceso, desde cualquier sitio y en } \\
\text { cualquier momento, a los entomos de aprendizaje disponibles en los centros docentes en los que estudien, teniendo en cuenta los principios de accesibilidad universal } \\
\text { y diseño para todas las personas y con pleno respeto a lo dispuesto en la normativa aplicable en materia de propiedad intelectual. }\end{array}$ \\
\hline
\end{tabular}

Elaboración propia 
En el Real Decreto 126/2014, de 28 de febrero, por el que se establece en el currículo básico de la Educación Primaria la competencia emprendedora, a través del espíritu emprendedor (Tabla 5), se encuentra como parte del objetivo de la educación primaria en el Artículo 7, y como elemento transversal en el Artículo 10. La asignatura troncal con la que se relaciona es Ciencias Sociales dentro del Bloque 3 referido a vivir en sociedad, a través de los contenidos, criterios de evaluación y estándares evaluables, y con la asignatura específica de valores sociales y cívicos en sus criterios de evaluación.

En este mismo marco legislativo encontramos que el ocio (Tabla 6) se relaciona con las asignaturas troncales de ciencias de la naturaleza en los bloques de: iniciación a la actividad científica; el ser humano y la salud y las tecnologías, objetos y maquinas, en todos ellos dentro de los estándares de aprendizaje. En la asignatura de primera lengua extranjera se refleja en los bloques de com-

Tabla 5. Presencia del emprendimiento en el currículo básico de primaria

\begin{tabular}{|c|c|c|c|c|}
\hline $\begin{array}{c}\text { TIPO DE } \\
\text { ASIG. }\end{array}$ & ASIG. & BLOQUE & APARTADO & СТTA \\
\hline & & & & $\begin{array}{l}\text { Articulo 7. Objetivos de la Educación Primaria. La Educación Primaria contribuirá a desarrollar en los niños y niñas las capacidades que } \\
\text { les permitan: b) Desarrollar hábitos de trabajo individual y de equipo, de esfuerzo y de responsabilidad en el estudio, asi como actitudes de } \\
\text { conflanza en si mismo, sentido critico, iniciativa personal, curiosidad, interés y creatividad en el aprendizaje, y espiritu emprendedor. }\end{array}$ \\
\hline & & & & $\begin{array}{l}\text { Articulo 10. Elementos transversales. Sin pejuicio de su tratamiento especifico en algunas de las asignaturas de cada etapa, la } \\
\text { comprensión lectora, la expresión oral y escrita, la comunicación audiovisual, las Tecnologias de la Información y la Comunicación, el } \\
\text { emprendimiento y la educación círica y constitucional se trabajarán en todas las asignaturas. 4. Los curriculos de Educación Primaria } \\
\text { incorporarán elementos curriculares orientados al desarrollo y afianzamiento del espiritu emprendedor. Las Administraciones } \\
\text { educativas fomentarán las medidas para que el alumnado paticipe en actividades que le permita afianzar el espiritu emprendedor y la } \\
\text { iniciativa empresarial a partir de aptitudes como la creatividad, la autonomia, la iniciativa, el trabajo en equipo, la confianza en uno mismo y } \\
\qquad \text { el sentido crítico }\end{array}$ \\
\hline \multirow[b]{7}{*}{ Troncal } & \multirow[b]{7}{*}{$\begin{array}{l}\text { Ciencias } \\
\text { sociales }\end{array}$} & & & $\begin{array}{l}\text { En las Ciencias Sociales se integran diversas disciplinas que estudian a las personas como seres sociales y su realidad en sus aspectos } \\
\text { geográficos, sociológicos, económicos e históricos. El objeto de las Ciencias Sociales en esta etapa es aprender a vivir en sociedad, } \\
\text { conociendo los mecanismos fundamentales de la democracia y respetando las reglas de la vida colectiva. A través de los contenidos } \\
\text { del Bloque 3, Vivir en sociedad, será posible iniciar un proceso de comprensión acerca de las formas de reconocer las características de } \\
\text { los distintos grupos sociales, respetando y valorando sus diferencias, quiénes son sus integrantes, cómo se distribuyen en el espacio } \\
\text { fisico, de qué manera se distribuye el trabajo entre sus miembros, cómo se producen y reparten los bienes de consumo, la vida } \\
\text { económica de los ciudadanos, la capacidad emprendedora de los miembros de la sociedad ye estudio de la empresa, que } \\
\text { comprenderá la función dinamizadora de la actividad empresarial en la sociedad, la organización social, politicay yerritorial y el } \\
\text { conocimiento de las instituciones europeas, la población, los sectores de producción, y educación financiera elemental. El área } \\
\text { fomenta el desarrollo de hábitos de trabajo individual y de equipo, además del esfuerzo como elemento esencial del proceso de } \\
\text { aprendizaje y de responsabilidad en el estudio, así como actitudes de confianza en sí mismo, sentido critico, iniciativa personal, } \\
\text { curiosidad, interés y creatividad en el aprendizaje y espíritu emprendedor. }\end{array}$ \\
\hline & & & Contenidos & Planificación y gestión de proyectos con el fin de alcanzar objetivos. Iniciativa emprendedora. \\
\hline & & & Criterios de Evaluación & $\begin{array}{l}\text { Desarrollar la creatividad y el espíritu emprendedor, aumentando las capacidades para aprovechar la información, las ideas y presentar } \\
\text { conclusiones innovadoras. }\end{array}$ \\
\hline & & & Estándares evaluables & $\begin{array}{l}\text { nuestra actitudes de confianza en sí mismo, sentido crítico, iniciativa personal, curiosidad, interés, creatividad en el aprendizaje y } \\
\text { espiritu emprendedor que le hacen activo ante las circunstancias que le rodean. }\end{array}$ \\
\hline & & \multirow{3}{*}{$\begin{array}{c}\text { Bloque } 3 \\
\text { vivir en } \\
\text { sociedad }\end{array}$} & Contenidos & Educación financiera. El dinero. El ahorro. Empleabilidad y espiritu emprendedor. La empresa. Actividad y funciones \\
\hline & & & Criterios de Evaluación & Comprender los beneficios que ofrece el espíritu emprendedor. \\
\hline & & & Estándares evaluables & $\begin{array}{l}\text { Desarrolla la creatividad y valora la capacidad emprendedora de los miembros de una sociedad. 14.1. Identifica diferentes tipos de } \\
\text { empresa según su tamaño y el sector económico al que pertenecen las actividades que desarrollan }\end{array}$ \\
\hline Especifica & \multicolumn{2}{|c|}{ Valores Sociales y civice } & Criterios de evaluación & Desarrollar la autonomía y la capacidad de emprendimiento para conseguir logros personales responsabilizándose del bien común \\
\hline
\end{tabular}

Elaboración propia basada en el Real Decreto 126/2014, de 28 de febrero, por el que se establece el currículo básico de la Educación Primaria 
prensión de textos orales; comprensión de textos escritos; producción de textos escritos, dentro de los apartados de contenidos y estándares de aprendizaje evaluables. En las asignaturas específicas se relaciona con Educación Física en la propuesta curricular, y en la asignatura de segunda lengua extranjera en los bloques de comprensión de textos orales en los apartados de estándares de aprendizaje evaluables.

Tabla 6. Presencia del ocio en el currículo básico de primaria

\begin{tabular}{|c|c|c|c|c|}
\hline TIPO DE ASIG. & ASIG. & BLOQUE & APARTADO & CITA \\
\hline \multirow{7}{*}{ TRONCAL } & \multirow{3}{*}{$\begin{array}{l}\text { Ciencias de la } \\
\text { Naturaleza }\end{array}$} & $\begin{array}{l}\text { 1. Iniciación a la actividad } \\
\text { cientifica }\end{array}$ & $\begin{array}{l}\text { Estándares de aprendizaje } \\
\text { evaluables }\end{array}$ & $\begin{array}{l}\text { Hace un uso adecuado de las tecnologías de la información y la comunicación como } \\
\text { recurso de ocio. }\end{array}$ \\
\hline & & $\begin{array}{l}\text { 2. El ser humano y la } \\
\text { salud }\end{array}$ & $\begin{array}{l}\text { Estándares de aprendizaje } \\
\text { evaluables }\end{array}$ & $\begin{array}{l}\text { Planifica de forma autónoma y creativa actividades de ocio y tiempo libre, individuales } \\
\text { y en grupo. }\end{array}$ \\
\hline & & $\begin{array}{l}\text { 5. La tecnologia, objetos } \\
\text { y máquinas }\end{array}$ & \begin{tabular}{|l|} 
Estándares de aprendizaje \\
evaluables
\end{tabular} & $\begin{array}{l}\text { Conoce y explica algunos de los avances de la ciencia en: el hogar y la vida cotidiana, la } \\
\text { medicina, la cultura y el ocio, el arte, la música, el cine y el deporte y las tecnologias de } \\
\text { la información y la comunicación. }\end{array}$ \\
\hline & \multirow{4}{*}{$\begin{array}{l}\text { Primera lengua } \\
\quad \text { Extran }\end{array}$} & \multirow{2}{*}{$\begin{array}{l}\text { 1.Comprensión de textos } \\
\text { orales }\end{array}$} & Contenidos & $\begin{array}{l}\text { Léxico oral de alta frecuencia (recepción) relativo a identificación personal; vivienda, } \\
\text { hogar y entorno; actividades de la vida diaria; familia y amigos; trabajo y ocupaciones; } \\
\text { tiempo libre, ocio y deporte; viajes y vacaciones; salud y cuidados fisicos; educación } \\
\text { y estudio; compras y actividades comerciales; alimentación y restauración; transporte; } \\
\text { lengua y comunicación; medio ambiente, clima y entorno natural; y tecnologías de la } \\
\text { información y la comunicación }\end{array}$ \\
\hline & & & $\begin{array}{l}\text { Estándares de aprendizaje } \\
\text { evaluables }\end{array}$ & $\begin{array}{l}\text { Comprende el sentido general y lo esencial y distingue los cambios de tema de } \\
\text { programas de televisión u otro material audiovisual dentro de su área de interés (p. e. en } \\
\text { los que se entrevista a jóvenes o personajes conocidos sobre temas cotidianos (por } \\
\text { ejemplo, lo que les gusta hacer en su tiempo libre) o en los que se informa sobre } \\
\text { actividades de ocio (teatro, cine, evento deportivo, etc.). }\end{array}$ \\
\hline & & $\begin{array}{l}\text { 3.Comprensión de textos } \\
\text { escritos }\end{array}$ & Contenidos & $\begin{array}{l}\text { Léxico escrito de alta frecuencia (recepción) relativo a identificación personal; vivienda, } \\
\text { hogar y entorno; actividades de la vida diaria; familia y amigos; trabajo y ocupaciones; } \\
\text { tiempo libre, ocio y deporte; viajes y vacaciones; salud y cuidados fisicos; educación y } \\
\text { estudio; compras y actividades comerciales; alimentación y restauración; transporte; } \\
\text { lengua y comunicación; medio ambiente, clima y entorno natural; y tecnologías de la } \\
\text { información y la comunicación }\end{array}$ \\
\hline & & $\begin{array}{l}\text { 4.Producción de textos } \\
\text { escritos: expresión e } \\
\text { interacción }\end{array}$ & Contenidos & $\begin{array}{l}\text { Léxico escrito de alta frecuencia (producción) relativo a identificación personal; } \\
\text { vivienda, hogar y entorno; actividades de la vida diaria; familia y amigos; trabajo y } \\
\text { ocupaciones; tiempo libre, ocio y deporte; viajes y vacaciones; salud y cuidados } \\
\text { fisicos; educación y estudio; compras y actividades comerciales; alimentación y } \\
\text { restauración; transporte; lengua y comunicación; medio ambiente, clima y entorno } \\
\text { natural; y tecnologías de la información y la comunicación. }\end{array}$ \\
\hline \multirow{3}{*}{ ESPECÍFICAS } & Ed. Física & Propuesta Curricular & & $\begin{array}{l}\text { El abanico de actividades de la propuesta curricular debe reflejar las manifestaciones } \\
\text { culturales de la sociedad en la que vivimos, que se manifiesta tanto en nuevas } \\
\text { formas de ocio como el turismo activo y las actividades de fitness o wellness, como } \\
\text { en los juegos y deportes, o en las manifestaciones artísticas. }\end{array}$ \\
\hline & \multirow[b]{2}{*}{$2^{\mathrm{a}}$ Lengua Extran } & $\begin{array}{l}\text { 1.Compresión de textos } \\
\text { orales }\end{array}$ & $\begin{array}{l}\text { Estándares de aprendizaje } \\
\text { evaluables }\end{array}$ & $\begin{array}{l}\text { Comprende el sentido general y distingue los cambios de tema de programas de } \\
\text { televisión u otro material audiovisual dentro de su área de interés (p. e. en los que se } \\
\text { entrevista a jóvenes o personajes conocidos sobre temas cotidianos, o en los que se } \\
\text { informa sobre actividades de ocio }\end{array}$ \\
\hline & & $\begin{array}{l}\text { 2. Producción de textos } \\
\text { orales: expresión e } \\
\text { interacción }\end{array}$ & $\begin{array}{l}\text { Estándares de aprendizaje } \\
\text { evaluables }\end{array}$ & $\begin{array}{l}\text { Hace presentaciones muy breves y sencillas, previamente preparadas y ensayadas, } \\
\text { sobre temas cotidianos o de su interés (presentarse y presentar a otras personas; dar } \\
\text { información básica sobre sí mismo, su familia y sus estudios; indicar sus aficiones e } \\
\text { intereses y las principales actividades de su dia a dia; describir brevemente y de manera } \\
\text { sencilla personas u objetos; decir lo que le gusta y no le gusta y dar su opinión usando } \\
\text { estructuras elementales). Se desenvuelve en situaciones muy básicas en, p. e., tiendas, } \\
\text { restaurantes o espacios de ocio }\end{array}$ \\
\hline
\end{tabular}

Elaboración propia basada en el Real Decreto 126/2014, de 28 de febrero, por el que se establece el currículo básico de la Educación Primaria 
Una vez establecida la presencia del ocio y la competencia emprendedora dentro de la educación formal obligatoria, vemos como se muestran en los documentos que fundamentan la configuración de las competencias docentes en el ámbito universitario, como institución responsable de su formación. En el caso del Libro Blanco del Título de Grado de Magisterio de la ANECA y la Orden ECI/3857/2007 sobre los requisitos para la verificación de los títulos de Maestro encontramos como el ocio (Tabla 7) se relaciona con el Grado de Maestro en Ed. Física como competencia específica en los conocimientos disciplinares, mientras que la competencia emprendedora (Tabla 8), a través del espíritu

Tabla 7. Competencias relacionadas con el ocio en la configuración del grado de magisterio

\begin{tabular}{|c|c|c|c|}
\hline GRADO & COMPETENCIAS & CONOCIMIENTOS & REFERENCIA OCIO \\
\hline \multirow{4}{*}{ ED. FÍSICA } & \multirow{4}{*}{$\begin{array}{l}\text { COMPETENCIAS } \\
\text { Específicas }\end{array}$} & $\begin{array}{l}\text { Conocimientos } \\
\text { disciplinares }\end{array}$ & $\begin{array}{l}\text { Conocer los aspectos que relacionan la actividad fisica con el ocio y la recreación para establecer } \\
\text { bases de utilización del tiempo libre: teatro, danza, deportes, salidas, etc. }\end{array}$ \\
\hline & & (SABER) & $\begin{array}{l}\text { Conocer las actividades de recreación y ocio, así como actividades alternativas y su aplicación } \\
\text { en el ámbito escolar }\end{array}$ \\
\hline & & \multirow{2}{*}{$\begin{array}{c}\text { Conocimientos } \\
\text { disciplinares (SABER } \\
\text { HACER) }\end{array}$} & Aplicar, programar y ejecutar actividades de ocio, tiempo libre y recreación en la escuela \\
\hline & & & $\begin{array}{l}\text { Conocer los aspectos que relacionan la actividad fisica con el ocio y la recreación para } \\
\text { establecer bases de utilización del tiempo libre: teatro, danza, deportes, salidas, etc }\end{array}$ \\
\hline
\end{tabular}

Elaboración propia basada en el Libro Blanco del Título de Grado de Magisterio Vol I y la Orden ECI/3857/2007

Tabla 8. Competencias relacionadas con el emprendimiento en la configuración del grado de magisterio

\begin{tabular}{|c|c|c|c|}
\hline LEGISLACIÓN & & & REFERENCIA EMPRENDIMIENTO \\
\hline $\begin{array}{l}\text { ORDEN ECI/3857/2007, de } 27 \text { de diciembre, por la que se } \\
\text { establecen los requisitos para la verificación de los títulos } \\
\text { universitarios oficiales que habiliten para el ejercicio de la } \\
\text { profesión de Maestro en Educación Primaria. }\end{array}$ & \multicolumn{3}{|c|}{$\begin{array}{l}\text { Apartado } 3 \text {. Objetivos. Competencias que los estudiantes deben adquirir: Colaborar con los distintos } \\
\text { sectores de la comunidad educativa y del entorno social. Asumir la dimensión educadora de la función } \\
\text { docente y fomentar la educación democrática para una ciudadanía activa. }\end{array}$} \\
\hline \multirow{5}{*}{ ANECA VOL. I } & \multirow{5}{*}{$\begin{array}{l}\text { COMPETENCIAS } \\
\text { TRANSVERSALES } \\
\text { SISTÉMICAS }\end{array}$} & \multirow{5}{*}{$\begin{array}{l}\text { Iniciativa y } \\
\text { espiritu } \\
\text { emprendedor }\end{array}$} & Maestro de ed. Infantil \\
\hline & & & Maestro de ed. Primaria, perfil de lengua extranjera \\
\hline & & & Maestros de educación primaria especialista en educación fisica \\
\hline & & & Maestros de educación primaria especialista en educación musical \\
\hline & & & $\begin{array}{l}\text { Maestros de educación primaria perfil de necesidades educativas } \\
\text { especiales }\end{array}$ \\
\hline
\end{tabular}

Elaboración propia basada en el Libro Blanco del Título de Grado de Magisterio Vol I y la Orden ECI/3857/2007 
emprendedor, es una de las competencias transversales sistémicas que tiene presencia en los Grados de Maestro de Educación Infantil, Lengua Extranjera; Educación Física; Especialista en Educación Musical y Perfil de Necesidades Educativas especiales

En cumplimiento con la normativa vigente, las universidades dentro de su autonomía configuran las Memorias de Grado para la obtención del Título de Maestro, adaptando a sus necesidades las especificaciones de la normativa. En el caso de la competencia emprendedora (Tabla 9), de las 19 memorias de grado analizadas se han encontrado referencias al emprendimiento en 6, de forma mayoritaria dentro de las competencias a alcanzar en las asignaturas. Las Universidades con mayor utilización de la competencia emprendedora en sus asignaturas son Alicante y La Rioja.

Tabla 9. Universidades con elementos referidos al emprendimiento

\begin{tabular}{|c|c|c|c|c|c|c|c|c|c|c|}
\hline & \multirow[b]{2}{*}{ ASIG. } & \multicolumn{4}{|c|}{ Elementos de Asignatura } & \multicolumn{4}{|c|}{ Tipo de Asig. } & \multirow{2}{*}{$\begin{array}{l}\text { Créditos } \\
\text { Totales }\end{array}$} \\
\hline & & Competenci & Resultados A & Contenidos & Criterios de & Básica & Obligatoria & Optativa & Otras & \\
\hline ALICANTE & 18 & 18 & 1 & & & 2 & 10 & 6 & & 120 \\
\hline ALMERÍA & 3 & 1 & & & 2 & & 3 & & & 38 \\
\hline BURGOS & 2 & 2 & & & & 1 & 1 & & & 10 \\
\hline CLM & 1 & & & 1 & & & & 1 & & 4,5 \\
\hline ISLAS BALEA & 8 & 8 & & & & & 5 & 3 & & 42 \\
\hline RIOJA & 18 & 18 & & & & 7 & 5 & 6 & & 234 \\
\hline
\end{tabular}

Elaboración propia basada en el análisis de las Memorias de Grado Verificadas

En el caso del ocio (Tabla 10), se han encontrado referencias en 12 universidades, siendo el elemento de la asignatura más utilizado a través de los contenidos. Por otro lado el tipo de asignatura con más alusiones al ocio es dentro de las optativas.

Tabla 10. Universidades con elementos referidos al ocio

\begin{tabular}{|c|c|c|c|c|c|c|c|c|c|c|}
\hline & \multirow[b]{2}{*}{ ASIG. } & \multicolumn{4}{|c|}{\begin{tabular}{|c|} 
Elementos de Asignatura \\
\end{tabular}} & \multicolumn{4}{|c|}{ Tipo de Asig. } & \multirow{2}{*}{$\begin{array}{l}\text { Créditos } \\
\text { Totales }\end{array}$} \\
\hline & & Competen & Resultados $A$ & Contenidos & Criterios de & Básica & Obligatoria & Optativa & Otras & \\
\hline Alicante & 1 & (2) & 1 & & & 1 & & & & 6 \\
\hline Almería & 1 & 1 & & & & 1 & & & & 6 \\
\hline Burgos & 1 & & & 1 & & & & 1 & & 5 \\
\hline CLM & 11 & 2 & 10 & 1 & & 2 & 2 & 7 & & 78 \\
\hline Extremadura & 1 & & & 1 & & & & 1 & & 6 \\
\hline Girona & 1 & & & 1 & & & & 1 & & 3 \\
\hline Granada & 3 & & 1 & 2 & & & 1 & 2 & & 24 \\
\hline Islas Baleares & 2 & & & 2 & & & & 2 & & 6 \\
\hline Jaén & 3 & & 1 & 2 & & & 2 & 1 & & 19 \\
\hline \begin{tabular}{|l|} 
La Rioja \\
\end{tabular} & 1 & 1 & & 1 & & & & & 1 & 22,5 \\
\hline Las Palmas GC & 2 & & 2 & & & & & & 1 & 10,5 \\
\hline Valladolid & 1 & 1 & & & & & & & & \\
\hline
\end{tabular}

Elaboración propia basada en el análisis de las Memorias de Grado Verificadas 
Si consideramos los créditos totales de las asignaturas en las que se refleja el emprendimiento y el ocio por cada una de las memorias de verificación analizadas, vemos como, aun teniendo más universidades la referencia al ocio, el emprendimiento forma parte de un mayor número de créditos (Figura 5). Si bien, en la Tabla 11 vemos como el ocio es considerado como asignatura de carácter optativo en 4 universidades.

Tabla 11. Universidades que contemplan el Ocio como asignatura

\begin{tabular}{|c|c|c|c|c|}
\hline & COMO ASIG. & ELEMENTO ASIG. & $\begin{array}{l}\text { TIPOLOGIAA } \\
\text { ASIG }\end{array}$ & CR \\
\hline Almería & Educación física, salud y ocio & $\begin{array}{l}\text { Contenidos: Educación para el ocio a través de la } \\
\text { actividad física }\end{array}$ & Optativa & 18 \\
\hline CLM & Juegos. Ocio y recreación & $\begin{array}{l}\text { Contenidos: Actividades motrices desarrolladas durante el } \\
\text { ocio y su aplicación al ámbito escolar } \\
\text { f. Gestión del ocio y organización de actividades } \\
\text { recreativas de naturaleza motriz fuera de la sesión de } \\
\text { Educación Física. }\end{array}$ & Optativa & 6 \\
\hline \multirow[b]{2}{*}{ Granada } & \multirow[b]{2}{*}{$\begin{array}{c}\text { Actividad física para el ocio y el } \\
\text { tiempo libre }\end{array}$} & $\begin{array}{l}\text { RESULTADOS DE APRENDIZAJE:Conocer los } \\
\text { beneficios de una adecuada distribución del ocio y el } \\
\text { tiempo libre en los alumnos. Saber desarrollar actividades } \\
\text { socio culturales, deportivas y de aire libre, tanto en entorno } \\
\text { urbano como en la Naturaleza. Aprender y diferenciar los } \\
\text { espacios y utilización de los mismos en el tiempo libre. } \\
\text { Comprometerse con la necesidad de adoptar un buen uso } \\
\text { del ocio y el tiempo libre en búsqueda de un estilo de vida } \\
\text { activo y saludables }\end{array}$ & & \\
\hline & & $\begin{array}{l}\text { CONTENIDOS: Conceptualización del Ocio y el Tiempo } \\
\text { Libre en la edad escolar. Gestión y planificación de } \\
\text { actividades en Tiempo Libre. Técnicas de dinamización. } \\
\text { Actividades físicas para el ocio y el tiempo libre. } \\
\text { Evaluación y ejecución de acciones relacionadas con el } \\
\text { ocio de los alumnos. }\end{array}$ & Optativa & 6 \\
\hline Islas Baleare & $\begin{array}{l}\text { Educación física, ocio y } \\
\text { recreación }\end{array}$ & $\begin{array}{l}\text { Resultados de aprendizaje: Conocer los aspectos que } \\
\text { relacionan la actividad física con el ocio y la recreación } \\
\text { para establecer bases de utilización del tiempo libre; } \\
\text { teatro, danza, salidas, deportas en el medio - Conocer las } \\
\text { actividades de recreación y ocio, así como actividades } \\
\text { alternativas y su aplicación en el ámbito escolar y } \\
\text { extraescolar para diseñar estrategias de promoción de la } \\
\text { actividad física en la escuela. } \\
\text { - Aplicar programar y ejecutar actividades de ocio, tiempo } \\
\text { libre y recreación en la escuela en los ciclos de primaria } \\
\text { para diseñar proyectos de animación. } \\
\text { - Orientar y supervisar las actividades relacionadas con la } \\
\text { actividad física y la recreación que se imparten en el } \\
\text { centro en horario extraescolar } \\
\text { escolar. }\end{array}$ & Optativa & 6 \\
\hline Jaén & $\begin{array}{l}\text { Actividades para el recreo, ocio } \\
\text { y tiempo libre }\end{array}$ & & Optativa & 6 \\
\hline
\end{tabular}

Elaboración propia basada en el análisis de las Memorias de Grado Verificadas 
Figura 5. Créditos totales de asignaturas con referencias al ocio y al emprendimiento por universidades

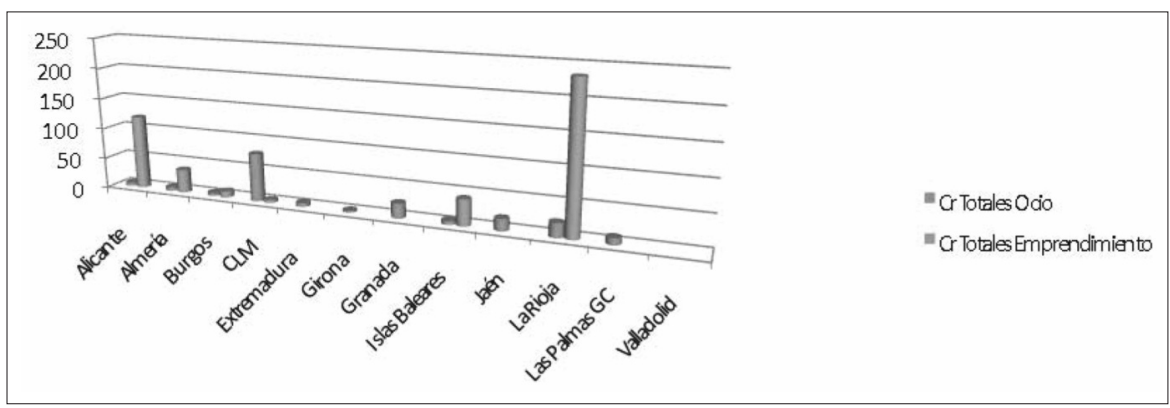

Elaboración propia basada en el análisis de las Memorias de Grado Verificadas

\section{CONCLUSIONES}

Los cambios profundos que se están produciendo en la sociedad afectan de manera directa a la educación, como transmisora de los principios y valores, así como promotora de los avances que en ella se han de producir. Las organizaciones internacionales señalan este momento como una oportunidad en la que definir nuevos paradigmas que fomenten el desarrollo sostenible siendo el pilar fundamental el ejercicio de la ciudadanía activa, participativa y democrática. El paradigma educativo emergente se sitúa dentro de los preceptos humanistas en los que la configuración del individuo ha de atender a todos y cada uno de los planos en los que se desenvuelve: intrapersonal, interpersonal, profesional y social (Avendaño, Paz y Parada, 2016; Bolivar, 2007; Comisión Europea, 2012; Mieles y Alvarado, 2012; Molina, 2015; OCDE, 2016/2016; Unesco, 2015/ 2016).

Es precisamente en esta nueva conceptualización donde la educación ha de determinar las competencias necesarias para alcanzar los fines marcados. Así, encontramos que se van sucediendo configuraciones de competencias de mayor complejidad y con un marcado carácter transversal y transferible, como son las competencias no cognitivas o las competencias clave, dentro de las cuales encontramos referencias explícitas tanto al emprendimiento (Agarwal, Audretsch y Sarkar, 2007; Grilo y Irigoyen, 2006; Krueger, 2015; Lackeus, 2015; O'Connor, 2013; Palmero et al., 2014; Seikkula-Leino, Ruskovaara, Ikavalko, Mattila, y Rytkola, 2010), como detección de oportunidades en el entorno con el fin de mejorar de forma individual y colectiva, como al ocio, como espacio en el que aplicar y aprender las habilidades (Bauman, 2007; Caride, 2012; 2014; 2010; 2011; Faché, 2002; Fernández, 2001; Mulford, 2010), dando satisfacción a la Hp. 1. 
Los ámbitos de adquisición de las competencias se han de realizar en entornos de aprendizaje flexible y abierto que permita el aprendizaje a lo largo de la vida, siendo los ámbitos educativos involucrados tanto la educación formal como la informal o no formal. No obstante, la educación humanista señala de forma clara cómo los aspectos relativos a la adquisición de las competencias se ha de realizar dentro de las etapas obligatorias, ya que la educación de calidad, inclusiva y equitativa se concibe como un derecho que ha de satisfacer las instancias sociales. De este modo, a través de la educación formal y obligatoria se garantiza el acceso de forma generaliza a las competencias necesarias (Hanushek y Kimko, 2000; Power, 2007). Si consideramos el emprendimiento y el ocio como parte del marco de referencia es en la etapa obligatoria donde se han de iniciar los procesos de adquisición, capacitando a las personas para su posterior desarrollo, así queda reflejado en su inclusión de la legislación educativa que regula los contenidos y objetivos de las etapas obligatorias. En este punto vemos reflejada la Hp. 2.

Si consideramos que la educación formal obligatoria ha de ser de calidad, el factor decisivo intraescolar que influye de forma directa y positiva en la calidad escolar y en el rendimiento de los alumnos es el docente (Angrist y Lavy, 2001; Darling-Hammond, Holtzman, Gatlin y Heilig, 2005; Decker, Mayer y Glazerman, 2004; Hanushek, Kain y Rivkin, 2004; OCDE, 2005; OCDE; 2010; Rivkin, 2005; Yoon, Duncan, Lee, Scarloss y Shapley, 2007), siendo la mejora del desempeño docente a través de la formación inicial y continua el objetivo que centra las políticas educativas, por su influencia en contexto educativo.

Las características propias de la sociedad hacen que la función docente y las expectativas que en ellos se depositan evolucionan también con los retos a los que deben de dar repuesta, que exigen que no sólo adquieran nuevas capacidades y conocimientos, sino su desarrollo continuo, lo que requiere de una formación inicial de calidad y un proceso coherente de desarrollo profesional (Ronfeldt y Reininger, 2012; Montalvo y Gorgels, 2013). En nuestro entorno, la función docente se apoya en tres pilares formativos: la formación inicial, la formación permanente y continua y la formación práctica. La formación permanente y práctica se considera dentro del desarrollo profesional continuo y está regulada por la legislación educativa de ámbito nacional y autonómico. Sin embargo, la formación inicial queda regulada por la legislación estatal dirigida al ámbito universitario, que goza a nivel organizativo y de gestión de mayor autonomía.

Tras los análisis realizados y en lo referido a la presencia de la competencia emprendedora y el ocio dentro de la formación inicial de los docentes en el ámbito universitario, hemos podido comprobar como en el ámbito legislativo 
y regulador ambas forman parte de configuración de las competencias que han de adquirir los docentes, no obstante en las memorias verificadas de grado estudiadas no tienen una presencia ni homogénea ni constante en los Grados de Magisterio, lo que nos lleva a ver que la $\mathrm{Hp} 3$ no se ve corroborada. A tenor de lo expuesto y, considerando la aplicabilidad práctica del estudio, creemos necesario promover el desarrollo de marcos globales de competencias profesionales para los docentes, definiendo de forma coherente los diferentes momentos de su adquisición, dotando a estos marcos de suficiente flexibilidad y capacidad de adaptación a la sociedad del conocimiento, tecnológicamente avanzada, donde la calidad, la innovación y transferencia de conocimientos es fundamental.

No debemos obviar la limitaciones propias de este estudio que a su vez se convierten en futuras líneas de investigación, ampliando el objeto de estudio a las universidades privadas, así como extendiendo el objetivo de investigación a los diferentes grados.

\section{REFERENCIAS BIBLIOGRÁFICAS}

Agarwal R., Audretsch D. B., Sarkar MB. (2007). The process of creative construction: Knowledge spillovers, entrepreneurship, and economic growth. Strategic Entrepreneurship Journal, 1(3-4), 263-286.

Akiba, M., LeTendre, G. K. y Scribner, J. P. (2007). Teacher quality, opportunity gap, and national achievement in 46 countries. Educational Administration Quarterly, 43 (4), 399-432.

Arroba, M. L. (2017). El tiempo libre de los niños. Anales de Pediatría Continuada, 7(6), 373-379.

Avendaño Castro, W.; Paz Montes, L. y Parada-Trujillo, A. (2016). The construction of citizenship: a model for its development in the school. AGO.USB. 16 (2), 359-678.

Bauman, Z. (2007). Tiempos líquidos. Vivir en una época de incertidumbre.. Madrid: Tusquets

Bolívar, A. (2007). Educación para la ciudadanía. Algo más que una asignatura. Barcelona: Graó.

Caballo, M. B., Caride, J. A. y Gradaille, R. (2012). Entre los tiempos escolares y los tiempos de ocio: su incidencia en la vida cotidiana de los adolescentes que cursan la Educación Secundaria obligatoria en España. Educar em Revista, 0 (45), 37-56.

Cáceres, P. (2003). Análisis Cualitativo de Contenido: una alternativa metodológica alcanzable. Psicoperspectivas, 2 (1), 53-82.

Cano García, E. (2007). Las competencias de los docentes. En A. López (Coord.), El desarrollo de competencias docentes en la formación del profesorado, 33-60. Madrid: MEC.

Caride, J. A. (2012). Lo que el tiempo educa: el ocio como construcción pedagógica y social. Arbor. Ciencia, Pensamiento y Cultura, 188 (754), 301-313. 
Caride, J. A. (2014). Del ocio como educación social a la pedagogía del ocio como desarrollo humano. Edetania, 45, 33-53.

Comisión Europea, (2012). Comunicación de la comisión al parlamento europeo. Un nuevo concepto de educación: invertir en las competencias para lograr mejores resultados socioeconómicos. SWD (2012) 371 final.

Cabeza, M. C., y Amigo, M. C. (2013). O encontro entre o ócio e a cultura: Reflexões sobre o ócio criativo desde a investigação empírica. Revista Lusófona de Estudos Culturais, 1(2), 4-27.

Cuenca, M. (2010). La fuerza transformadora del ocio. En M. Cuenca, E. Aguilar y C. Ortega (Eds.), Ocio para innovar. Bilbao: Universidad de Deusto. 17-82

Cuenca, M. (2011). El ocio como ámbito de Educación Social. Educación Social. Revista de Intervención Socioeducativa, 47, 25-40.

Darling-Hammond, L., Holtzman, D. J., Gatlin, S. J., y Heilig, J. V. (2005). Does teacher preparation matter? Evidence about teacher certification, Teach for America, and teacher effectiveness. Education Policy Analysis Archives, 13(42), 1-51. Disponible en web: http://epaa.asu.edu/epaa/v13n42/. Recuperado el día 1 de febrero de 2017http:// epaa.asu.edu/epaa/v13n42/

EACEA/Eurydice, (2012). Entrepreneurship Education at School in Europe: National Strategies, Curricula and Learning Outcomes. Brussels: EACEA/Eurydice.

Esteve, J. M. (2004). La profesión docente en Europa: perfil, tendencias y problemática. La formación inicial. Revista de Educación, 340, 19-40.

Faché, W. (2002). El aprendizaje como medio para disfrutar del ocio y el aprendizaje como fin del ocio. En C. De la Cruz (Ed.), Educación del Ocio. Propuestas internacionales (pp. 69-83). Bilbao: Universidad de Deusto.

Fernández, M. (2001). La jornada escolar. Análisis y valoración de los procesos, los efectos y las opciones de la implantación de la jornada continua. Barcelona: Ariel.

Ganuza Fernández E. y Francés García F. J. (2015). Citizen participation in europe: a comparative analysis from the sociopolitical contexts. OBETS. Revista de Ciencias Sociales, 10 (1), 235-260.

Glatthorn, A. A., Boschee, F. A., y Whitehead, B. M. (2006). Curriculum leadership: development and implementation. Londres: Sage publications.

Grilo I. e Irigoyen J. M. (2006). Entrepreneurship in the EU: To wish and not to be. Small Business Economics, 26 (4), 305-318.

Hanushek, E., Kain, J. y Rivkin, S. (2004). Why Public Schools Lose Teachers. The Journal of Human Resources, 34 (2), 326-356.

Hanushek, E. y Kimko, D. (2000). Schooling, labor force quality, and the growth of nations. American Economic Review, 90 (5), 1184- 1208.

Imbernón, F. (2004). La profesión docente desde el punto de vista internacional ¿qué dicen los informes?. Revista de Educación, 340, 41-49.

Krueger, N. F. (2015). Entrepreneurial Education in Practice. Part 1: The Entrepreneurial Mindset, Entrepreneurship360 Thematic Paper, Paris, OECD, Disponible en web: http://www.oecd.org/cfe/leed/Entrepreneurial-Education-Practice-pt1.pdf. Recuperado el 29 de enero de 2017. 
Lackeus, M. (2015). Entrepreneurship in education. What, Why, When, How. OCDE: París. Martín del Pozo, R., Fernández-Lozano, P., González-Ballesteros, M., y De Juanas, A. (2013). El dominio de los contenidos escolares: competencia profesional y formación inicial de maestros. Revista de Educación, 360, 363-387.

Mella, O. (2003). Metodología cualitativa en ciencias sociales y educación: orientaciones teórico-metodológicas y técnicas de investigación. Santiago: Primus.

Mieles Barrera, M. D. y Alvarado Salgado, S. V. (2012). Ciudadanías y competencias ciudadanas. Estudios políticos, (40), 53-75.

Molina, A. (2015). Proceso de formación para una ciudadanía activa. Revista Electrónica de Investigación Educativa, 17(1), 1-4.

Montalvo, J. y Gorgels, S. (2013). Calidad del Profesorado, Calidad de la Enseñanza y Aprendizaje: Resultados a partir del TEDS-M. En INEE (Ed.), TEDS-M: Informe español Vol II.

Morán de Castro, M. C. (2012). Incidencia de la jornada escolar en los procesos de socialización infantil. Educar em Revista, Curitiba, Brasil, 45, 19-36.

Morán, M. C. y Varela, L. (2011). Percepciones del profesorado de educación primaria sobre la conciciliación de los tiempos escolares y familiares. Barcelona: XII Congreso Internacional de Teoría de la Educación. Universidad de Barcelona. Disponible en web: http://www.ub.es. Recuperado el día 14 de febrero de 2017.

Mulford, B. (2010). Leadership for improving the quality of secondary education. Some international development. En B. Pont, Liderazgo y autonomía del centro escolar: perspectivas internacionales Participación educativa, 13, 62-72.

OCDE, (2005). Teachers Matter Attracting, Developing and Retaining Effective Teachers. París: OCDE.

OCDE, (2010). Evaluación y reconocimiento de la calidad de los docentes. Prácticas internacionales. París: OCDE

OCDE, (2016). Education at a Glance 2016 OECD Indicators. París: OECD. Disponible en web: http://www.oecd.org/edu/education-at-a-glance-19991487.htm. Recuperado el 3 de enero de 2017.

OCDE, (2016). Trends Shaping Education, 2016. París: OECD. Disponible en web: http://www.oecd-ilibrary.org/education/trends-shaping-education-2016_trends_ edu-2016-en. Recuperado el 30 de diciembre de 2016.

O'Connor, A. (2013). A conceptual framework for entrepreneurship educationpolicy: Meeting government and economic purposes. Journal of Business Venturing, 28, 546-563.

Pesquero, E., Sánchez, E., González, M., Martín, R., Guardia, S., Cervelló, J.; Fernández P.; Martínez M. y Varela P. (2008). Las competencias profesionales de los maestros de Primaria. Revista Española de Pedagogía, 241, 447-466

Palmero C., Escolar C., Luis I., De la Torre T., Gañán A., Baños V., Corbi M. y Jiménez A. (2014). Impacto de la educación en el emprendimiento. Making-of de un análisis por grupos de discusión. International Journal of Developmental and Educational Psychology, 7(1), 507-518. 
Power, S. (2007). Policy-relevant synthesis of results from European research in the field of Education. Comisión Europea, Dirección General de Investigación.

Raffini, L. (2015). Introducción. Una práctica de reflexión sociológica más allá de los marcos nacionales. OBETS. Revista de Ciencias Sociales, 10 (1), 2015,17-35.

Rivkin, S., Hanushek, E. y Kain, J. (2005). Teachers, Schools, and Academic Achievement. Econometrica, 73 (2), 417-458.

Ronfeldt, M. y Reininger M. (2012). More of better student teaching?. Teaching and Teacher Education, 28, 1091-1106.

Santelices Etchegaray, M.V.yValenzuela Rojas, F. (2015). Importancia de las características del profesor y de la escuela en la calidad docente:Una aproximación desde la Teoría de Respuesta del Ítem.Estudio. Pedagogía, 41 (2), 233-254.

Seikkula-Leino, J., Ruskovaara E., Ikavalko M., Mattila J., y Rytkola T. (2010). Promotingentrepreneurshipeducation: the role of the teacher? Education+ Training, 52, 117-127.

Strauss, A. y Corbin, J. (2002). Bases de la investigación cualitativa. Técnicas y procedimientos para desarrollar la teoría fundamentada. Antioquía: Universidad de Antioquía.

UNESCO, (2015). Rethinking Education: Towards a global common good?. París: UNES$\mathrm{CO}$.

UNESCO, (2016). La educación al servicio de los pueblos y el planeta: Creación de futuros sostenibles para todos. París: UNESCO.

Yoon, K. S., Duncan, T., Lee, S. W.-Y., Scarloss, B. y Shapley, K. (2007). Reviewing the evidence on how teacher professional development affects student achievement, Issues $\mathcal{E}$ Answers, REL 2007-No. 033, Institute of Education Sciences, National Center for Education Evaluation and Regional Assistance, Regional Educational Laboratory Southwest, U.S. Department of Education, Washington, D.C., https://ies.ed.gov/ ncee/edlabs/regions/southwest/pdf/REL_2007033_sum.pdf

Zabalza, M. A. (2004). Buscando una nueva hoja de ruta en la formación del profesorado. Revista de Educación, 340, 51-58.

MARÍA ISABEL LUIS RICO, miluis@ubu.es, Orcid 0000-0001-5857-9404. Doctora en Ciencias de la Educación. Licenciada en Pedagogía. Profesora Ayudante Doctor en la Universidad de Burgos. Miembro de los grupos de investigación internacional Formadesa, Ociogune y Resortes. Sus investigaciones principales se centran en el aprendizaje a lo largo de la vida, formación universitaria para personas mayores y emprendimiento.

TAMARA DE LA TORRE CRUZ, tdtorre@ubu.es. Orcid 0000-0003-4484-8110. Doctora en Ciencias de la Educación. Licenciada en Pedagogía. Especialista en Terapias Corporales. Profesora Ayudante Doctor en la Universidad de Burgos. Miembro del grupo de investigación Formadesa. Sus investigaciones se cen- 
tran en el aprendizaje a lo largo de la vida, formación universitaria para personas mayores y emprendimiento.

ALFREDOJIMÉNEZ PALMERO, alfredo.jimenez@kedgebs.com. Tiene una licenciatura, master y doctorado en Administración de Empresas. Actualmente es Profesor Titular en Kedge Business School (Burdeos, Francia). Su interés investigador se centra en el proceso de internacionalización. Además, ha sido profesor visitante en diversos centros de educación superior en Australia, Dinamarca, Ecuador, Italia, México, Noruega y Singapur.

Recibido: 29/03/2017

Aceptado: 14/09/2017 



\title{
RELACIÓN ENTRE LAS PRÁCTICAS DE OCIO SIGNIFICATIVAS DE LA JUVENTUD Y EL EMPRENDIMIENTO SOCIAL. EL CASO THINK BIG JÓVENES \\ THE RELATIONSHIP BETWEEN YOUTH'S SIGNIFICANT LEISURE PRACTICES AND SOCIAL ENTREPRENEURSHIP. THE THINK BIG JÓVENES CASE
}

\author{
Amaia Makua Biurrun \\ Universidad de Deusto, España \\ amaia.makua@deusto.es \\ Macarena Cuenca-Amigo \\ Universidad de Deusto, España \\ macarena.cuenca@deusto.es

\section{Roberto San Salvador del Valle Doistua \\ Universidad de Deusto, España roberto.sansalvador@deusto.es}

\section{Cómo citar / Citation}

Makua Biurrun, Amaia; Cuenca-Amigo, Macarena y San Salvador del Valle Doistua, Roberto (2017) "Relación entre las prácticas de ocio significativas de la juventud y el emprendimiento social. El caso Think Big Jóvenes”. OBETS. Revista de Ciencias Sociales, 12(Extra 1): pp-pp. 151-176.

doi:10.14198/OBETS2017.12.1.16

\section{Resumen}

La crisis iniciada en 2008 dificulta el desarrollo personal y social de la juventud española y le aleja del ejercicio de una ciudadanía juvenil. Una de las respuestas institucionales para aliviar sus efectos es el fomento del emprendimiento social, como el Programa Think Big Jóvenes de la Fundación Telefónica. Por otra parte, el ocio significativo está presente en este grupo de edad y puede ser fuente de iniciativas comunitarias transformadoras. El objetivo general de 
este artículo es explorar el binomio ocio y emprendimiento social de los jóvenes españoles a través de un análisis teórico que se completa con el análisis empírico de la experiencia de 65 jóvenes integrantes de Think Big en el año 2014. Los resultados confirman que dicha relación subyace.

Palabras clave: Juventud; emprendimiento social; innovación; ocio; creatividad; Think Big Jóvenes.

\begin{abstract}
The crisis that began in 2008 hinders Spanish young's personal and social development and keeps them from playing an active role according to the notion of a youth citizenship. One of the institutional responses to alleviate its effects is the promotion of social entrepreneurship. One of them is Think Big Jóvenes initiative, promoted by Fundación Telefónica. On the other hand, significant leisure is present among young, and can be a source of transformational community initiatives. The general aim of this text is to explore the binomial leisure and social entrepreneurship of young Spaniards based on a theoretical analysis that is completed with the empirical analysis of the experience of 65 young members of Think Big in 2014.
\end{abstract}

Keywords: Youth; social entrepreneurship; innovation; leisure; creativity; Think Big Jóvenes.

\title{
Extended Abstract
}

The crisis has been particularly hard in Spain since 2008. Its economic and social consequences have particularly concerned to certain groups of population, like young. Institutions fear a lost generation of young people to whom access to a first job is denied. To alleviate this severe situation initiatives focused on social entrepreneurship are implemented. One of them is Think Big Jóvenes, which is defined on its webpage as "a training program by Telefónica Foundation, which gives you the opportunity to develop skills of social entrepreneurship and turn an idea into a project that will enable you to change the world," (Fundación Telefónica, s.f.). Social entrepreneurship, which seems to be in accordance with young's ideals of changing the status quo, is seen as a way to improve the bad situation. This type of entrepreneurship is one of the pillars of our analysis. Another one is the significant leisure experiences of this group of social innovators. Leisure is a key factor for youth and it is connected with their identity and with their social development.

The general objective of this study is to explore the binomial leisure and entrepreneurship. This main objective is deployed in four specific objectives: (1)to analyse whether it exists or not a relationship between the perceived probability that the Think Big project becomes an enterprise and the connection of the Think Big project with any activity of leisure of the respondent; (2) to analyse whether it exists or not a relationship between the perceived probability that the Think Big project becomes an enterprise and the connection of the Think Big project with a significant activity of leisure of the respondent; (3) to analyse the relationship between the involvement of the respondent with his/her significant activity of leisure and the connection of the pro- 
ject Think Big with such significant leisure activity; and (4) to describe the activities of significant leisure that are inspiring the entrepreneurship projects analysed.

This text is based on a review of the scientific literature related to the situation of the Spanish youth in the current crisis, to the notion of social entrepreneurship and to the concept of meaningful leisure. The theoretical analysis is completed with the study of a real case, the Think Big Jóvenes initiative promoted by Telefónica Foundation. Questionnaires were distributed among the participants in the month of July of 2014. We obtained 65 responses, which have provided the basis for the description and discussion of the results. This article ends with a series of conclusions.

The theoretical review leads us to confirm certain ideas. The crisis that still remains in Spain has a direct impact on young people, who see diminished their chances of personal development and integration in social life. Today, more than ever, measures that make effective the concept of youth citizenship are needed. The idea of youth citizenship recognize young are the protagonists of their citizen status. They are supposed to conquer spaces of autonomy and participation (Benedict, 2015). Entrepreneurship, and in particular social entrepreneurship, is one of the possible ways to promote the occupation of individual and community spaces for young people. In line with those who understand that entrepreneurship is more than a mere economic phenomenon, young appreciate that it can become an attitude to life and probably for a long life. It happens to be a way that allows youth to take advantage of the opportunities that the crisis offers them. This attitude responds to the emerging social demand of entrepreneurial skills that lead to innovation and to a more participatory and democratic society.

Social entrepreneurship is a way to launch innovative initiatives that transform society. Those who get on the adventure of putting into practice projects of this kind show high levels of intrinsic motivation and creativity. These two factors are also determinants in serious and significant leisure practices. This kind of leisure-more transcendent and challenging-also engages youth and could be a manner to connect them with collective improvement projects.

The results of this study show that those participants with Think Big projects related to their significant leisure activities perceived a higher probability that their project would become their own enterprise (Objective 2) than the rest. However, such relationship did not occur with any leisure activity (Objective 1), but only with significant leisure activities. This idea is of great interest and is consistent with the theories coming from the Leisure Studies, like for example, the serious leisure of Stebbins (2008). Precisely, this author (Stebbins, 2010) proposes that analysing social entrepreneurship from the perspective of serious leisure enriches the understanding of the phenomenon. He considers that social entrepreneurship is a practice of serious and non-casual leisure.

Consistently with the above mentioned, it was also shown that young with Think Big projects connected to their significant leisure activities presented a greater involvement with their meaningful leisure activities than the rest. 
In a way, a significant leisure activity that becomes an entrepreneurship project happens to become a job. Although this could seem contradictory, people can experience flow at work and it is precisely in these cases when lines between work and leisure are blurred (Stebbins, 2010).

Finally, considering these significant leisure practices taking into account the dimensions of leisure suggest that when meaningful activities belong to the creative and solidarity sphere, they might lead more likely to a social entrepreneurship project. However, the relationship between variables has not been confirmed in this research due to the limited sample size. So caution must be applied, as the findings might not be transferable. However, it seems that this line of research could be explored in depth in the future, since there is literature that supports it (Rivera and Santos, 2016).

The findings of this study have implications for both future interventions and research. On the one hand, in terms of practice, social entrepreneurship programs -like Think Big- could foster contacts and alliances with clubs or associations of amateurs or volunteers, since these groups are clear practitioners of serious leisure. The dissemination of the entrepreneurship programmes among these groups might help to increase the number of social projects that finally become companies. On the other hand, and taking into account the limitations derived from the size of the aforementioned sample, this exploratory study opens the door to future investigations which, both, corroborates the greater involvement of those who implement a social project connected with their significant leisure practices and examine the relationship between the different types of leisure activities and their central role in projects of social entrepreneurship.

Finally, we highlight the beneficial role that initiatives such as Think Big can play, especially for young people who are developing a social enterprise based on a significant leisure activity. The experiences of these young will become part of their leisure capital for the rest of their lives.

\section{INTRODUCCIÓN}

La crisis se ha dejado sentir en España de un modo intenso desde 2008. Las repercusiones económicas y sociales perjudican especialmente a determinados colectivos, entre ellos los jóvenes (Benedicto, 2016a). Ante el temor de que se dé una generación perdida de jóvenes sin acceso a su primera oportunidad laboral, desde diversos organismos se ponen en marcha iniciativas que buscan a través del emprendimiento paliar esta grave situación. Una de estas iniciativas es Think Big Jóvenes, que en su página web se define como "un programa formativo de Fundación Telefónica que te ofrece la oportunidad de desarrollar habilidades de emprendimiento social y convertir una idea en un proyecto con el que cambiar el mundo" (Fundación Telefónica, s.f.). La inserción de sus destinatarios se lleva a cabo a través del emprendimiento social, acorde con el espíritu inherente a la juventud, deseosa de cambiar el status quo. Esta moda- 
lidad de emprendimiento es uno de los ejes de nuestro análisis, que se complementa con la consideración del ocio significativo de este grupo de innovadores sociales. El ocio constituye un factor clave en la identidad y desarrollo social de los jóvenes y puede ser además una fuente que inspira a personas emprendedoras. El binomio ocio-emprendimiento ha sido objeto de estudio por Williams y Nadin (2012) quienes afirman que además de las destrezas que pueden desarrollarse a través de un empleo, los intereses personales y las aficiones pueden ser un motor claro para la puesta en marcha de un proyecto. La relación existente entre el ocio significativo de los jóvenes y la puesta en marcha de iniciativas emprendedoras de corte social inspiran este artículo. En él llevamos a cabo una revisión de la literatura científica relacionada con la situación de la juventud española en la crisis, con la noción de emprendimiento social y de ocio significativo. El análisis teórico se completa con el estudio de un caso real, la iniciativa Think Big Jóvenes de la Fundación Telefónica a través de un cuestionario distribuido entre sus participantes en los meses de julio y septiembre del 2014. Las 65 respuestas obtenidas sirven de base para la descripción de los resultados y de su discusión. Este artículo se cierra con una serie de conclusiones.

\section{EL IMPACTO DE LA CRISIS EN LA JUVENTUD ESPAÑOLA}

La crisis que se inicia en 2008 afecta al colectivo de jóvenes de un modo especialmente acusado en España. La Encuesta de Población Activa del cuarto trimestre del 2016 arroja una tasa de paro media del 42,92\% para el segmento de 16 a 24 años y corrobora la precariedad del empleo al que optan con un $74 \%$ de los contratos temporales (INJUVE, 2016). La juventud se encuentra en una situación de debilidad en un mercado laboral para el que están sobrecualificados y del que reciben salarios bajos (Gentile, 2015:36). Esta realidad resulta aún más dolorosa al compararla con el entorno europeo, donde España encabeza las estadísticas de paro juvenil e incluso duplica la media de la Unión Europea (Morales y Montes, 2013). Consecuentemente, estos años de escasez y recortes modifican las perspectivas de la juventud española acerca de su integración social. A pesar de ser la mejor formada de la historia del país, vive el presente con inseguridad y prevé un futuro inestable. Las circunstancias difíciles provocan transformaciones importantes en el proceso de transición de la juventud a la vida adulta (Alcañiz y Querol, 2015; Gentile, 2015). La huella se deja sentir en el plano subjetivo: la situación se vive negativamente, como una amenaza no sólo al nivel de vida y consumo, sino incluso a las mismas posibilidades de supervivencia y retrotrae a la juventud a valores más materialistas que en décadas anteriores (Hernández, Parra y Pérez, 2016). 
En un horizonte tan poco halagüeño urge promover desde el tejido institucional alternativas paliativas que permitan desarrollar la noción de ciudadanía juvenil, entendida como

un proceso de conquista de espacios de autonomía (personal y colectiva) e implicación participativa de los jóvenes que dejarían así de ser mero objeto pasivo de la actuación pública para convertirse en sujetos protagonistas de unas políticas activas de promoción de su condición ciudadana (Benedicto, 2016b:926)

Lograr la efectividad de este concepto requiere conectar intrínsecamente la capacidad de los jóvenes de "orientar y gestionar su propio recorrido biográfico con el ejercicio de su condición ciudadana desde una dimensión activa y participativa" (Benedicto, 2016b:926).

Rivera y Santos (2016) analizan la relación existente entre los estilos de vida de niños y adolescentes españoles e italianos con su participación cívica y política y constatan que dicha implicación comunitaria es un espejo del capital social y de la sociabilidad y empoderamiento personal de estos grupos poblacionales. Asimismo recuerdan su baja participación en proyectos comunitarios en Europa, especialmente en los países del Sur como España. De ahí la justificación de su análisis, centrado en los predictores de la participación social de niños y adolescentes relacionados con sus estilos de vida: diálogo familiar, comportamientos de riesgo, actividades culturales, valores cívicos, supervisión familiar y relaciones grupales entre pares. Concluyen que los poderes públicos encargados de promover programas que incentiven la participación cívica y política de los más jóvenes deben considerar especialmente la relación positiva que se establece con algunos de estos factores, como el diálogo familiar, los comportamientos de riesgo y la realización de actividades culturales.

Entre las varias opciones, el emprendimiento puede ser una que permita la conquista de nuevos espacios de autonomía comunitarios para la juventud. En términos generales los poderes públicos europeos, y también los españoles, han tratado durante estos años de dar respuesta a las consecuencias de la crisis con reformas en el mercado laboral, dirigidas a incrementar la productividad y reintegrar a las personas desempleadas. Ante la predominante restricción en el gasto público se han alzado algunas voces críticas por entender que suponían una prolongación de la situación, apostando por medidas más expansionistas (Aceleanu, 2013). En sintonía con esta idea, una de las líneas de trabajo destacada en Europa en los últimos tiempos ha consistido en favorecer la creación de nuevas empresas. El emprendimiento suscita cada vez más interés social $y$, aunque probablemente no sea una panacea, seguramente puede ser parte de la solución a los retos que se plantean, también para la juventud. Con esta 
apuesta institucional se busca reducir la tendencia a considerar el emprendimiento como una "opción minoritaria como medio de inserción laboral, más aún entre la población joven" (Morales y Montes, 2013:15). En opinión de Rivera y Santos (2015) dichas iniciativas debieran incorporar metodologías para promover un cambio en pro de actitudes más positivas hacia el emprendimiento entre la juventud española. Argumentan que es necesario generar un emprendimiento de calidad basado en oportunidades, más que en meras necesidades, que dé lugar a iniciativas estables cuyo motor sea la innovación y la competitividad y que aspiren a un horizonte de crecimiento. Este tipo de proyectos son los que aportan mayores beneficios económicos y sociales y los que logran generar un cambio social real.

Antes de seguir avanzando en nuestro razonamiento, debemos subrayar que el emprendimiento es un fenómeno multidimensional y vivo, sobre el que no existe unanimidad en el ámbito científico (Rivera, 2011). Centrándonos en el sentido que la juventud le atribuye, éste no se ciñe a la mera puesta en marcha de un proyecto económico. En palabras de Morales y Montes (2013), los jóvenes entienden que "es un concepto amplio que va más allá que la idea de generar empresas, es una postura frente a la vida y, muy posiblemente, de por vida" (p.32). La percepción positiva de algunos jóvenes hacia el emprendimiento descansa en la lectura de la crisis como una oportunidad, más que como un obstáculo o una trampa. A pesar de las actuales dificultades reivindican su lugar en la sociedad y se activan "para buscar sus soluciones, encontrar oportunidades nuevas, caminos alternativos y, eventualmente, originales que ellos mismos elijan perseguir" (Gentile, 2015:51).

Esta visión concuerda con la creciente demanda social de capacidades emprendedoras, "desde una perspectiva amplia y no sólo como la posibilidad de crear empresa, sino también de instituciones que contribuyan a la innovación económica y social" (Morales y Montes, 2013:33). La integración estratégica de la necesidad de generar una capacidad emprendedora pasaría por "alcanzar una sociedad más participativa y más democrática" (p.33). Así, el emprendimiento social podría servir a una doble finalidad: por un lado, dar respuesta a las cada vez mayores reivindicaciones de innovación de la sociedad; y, por otro, contribuir a la construcción de la ciudadanía juvenil. La innovación social encuentra acomodo y se define en la Unión Europea como

....el desarrollo y la implementación de nuevas ideas (productos, servicios y modelos) para satisfacer las necesidades sociales y crear nuevas relaciones o colaboraciones.... Su objetivo es mejorar el bienestar humano.... son innovaciones no únicamente buenas para la sociedad, también potencian la capacidad de actuar de los individuos (European Commission, 2013:6) 
Dicha innovación puede asimismo lograrse por otras vías distintas al emprendimiento social generado por empresas, tales como iniciativas de organizaciones, instituciones o de la sociedad en su conjunto (Alonso, González y Nieto, 2015).

\section{EL EMPRENDIMIENTO SOCIAL}

La definición de emprendimiento social no es unitaria, si bien reina cierto consenso sobre algunos de sus rasgos más característicos. Diversos autores coinciden en que el emprendimiento social se orienta a la búsqueda de soluciones a problemas sociales que afectan a la persona y al planeta (Bosma, Schøtt, Terjesen, y Kew, 2016; Guzmán y Trujillo, 2008; Neck, Brush y Allen, 2009), al deseo de hacer del mundo un lugar mejor (Katzenstein y Chrispin, 2011). El emprendimiento social tiene una misión social explícita que constituye su principal razón de ser (Timmons, 2009). Su desarrollo requiere "la acción directa de los emprendedores sociales" así como "la modificación de manera permanente al desequilibrio social identificado en el entorno" (Guzmán y Trujillo, 2008: 120). Estos mismos autores resaltan cómo en contraste con el emprendimiento de negocios los problemas se abordan desde una óptica social y no necesariamente motivada por remuneraciones materiales. Además, lo que para el emprendimiento de negocios supone una falla de mercado, puede ser una oportunidad para el social. Para Timmons (2009) otra diferencia radica en que ambos funcionan en entornos diferentes y regidos por distintas reglas.

A pesar de las divergencias, ambas modalidades, la empresarial y la social, requieren de respuestas creativas en hábitats cada vez más complejos e inciertos (Schwagler, 2014). La creatividad es pues un elemento esencial en cualquier iniciativa emprendedora, si bien su exigencia suele ser mayor para quienes emprenden un proyecto altruista y social. Un estudio llevado a cabo por Weitzel, Urbig, Desai, Sanders y Acs (2010) establece que quienes poseen capacidades para emprender y son más creativos se comportan de un modo más altruista. La creatividad va de la mano de la innovación y el emprendimiento. La creatividad supone producir soluciones nuevas y apropiadas a problemas no zanjados en cualquier dominio de la actividad humana. La innovación es la implementación de tales ideas novedosas y apropiadas. Y el emprendimiento es una forma particular de innovación, la implementación exitosa de ideas creativas para producir nuevos negocios o para ponerlas en práctica en un negocio ya existente (Amabile, 1996). Esta experta acuña el término creatividad emprendedora a la que necesariamente vincula elevados grados de motivación intrínseca. En contraste con la motivación extrínseca, en la que se busca una recompensa externa, cuando se actúa intrínsecamente se hace por el mero placer que la actividad en sí misma produce (Csikszentmihalyi, 1975). Dicha creati- 
vidad tiene más probabilidades de materializarse en un proyecto de corte social si va acompañada de la capacidad de establecer relaciones interpersonales (Rivera y Santos, 2015) ya que las personas agrupadas tienen más facilidad para generar soluciones creativas (Amabile, 1997). De hecho, tal y como subraya Hennessey (2015), los procesos creativos no pueden ocurrir sin interacción humana, ya sea presente o pasada, ni sin influencia social.

La justificación del emprendimiento social radica en que, aunque hoy el crecimiento económico es aún necesario, no es suficiente. Por ello se erige como la máquina del cambio positivo y sistémico que modificará lo que hacemos, cómo lo hacemos y los motivos que nos llevan a hacerlo (Neck, Brush y Allen, 2009). En palabras de Rivera y Santos (2015) constituye un "elemento fundamental de la innovación social" (p. 15). De ahí que el cambio que supone el emprendimiento social no tiene tanto que ver con un cambio que rompe patrones, sino con las personas que rompen con los patrones (Light, 2009).

El emprendimiento social suele asociarse con jóvenes generadores de cambio, que son idealistas por naturaleza. Esta afirmación se confirma a través del estudio desarrollado por Global Entrepreneurship Monitor (Bosma et al, 2016) sobre esta modalidad de emprendimiento. El segmento de 18 a 34 años, presenta un mayor número de nuevos emprendedores sociales que de negocios en tres regiones del mundo, entre las que se encuentra Europa Occidental. Tomando en consideración iniciativas, organizaciones o actividades ya operativas, éstas son más numerosas en prácticamente todas las regiones del mundo, salvo en Latino América y el Caribe. El análisis de indicadores en una muestra de 58 economías mundiales retrata a España como uno de los países europeos con menores niveles de implicación en actividades de emprendimiento social, tanto en fase inicial como operativa, sólo por delante de Bulgaria (Bosma et al, 2016).

Una vez aclarada nuestra comprensión del concepto de emprendimiento social, nos aproximamos a la experiencia de ocio valiosa para poder analizar su incidencia en el emprendimiento social de la juventud española.

\section{EL OCIO VALIOSO}

El ocio vivido en esta etapa vital resulta crucial, pues será la base del capital de ocio-competencias, gustos e intereses- de los jóvenes durante el resto de sus vidas (Roberts, 2012) especialmente si dichas aficiones "alcanzan un determinado nivel de competencia técnica y dan acceso a disfrutes que se abren hacia retos de superación" (Cuenca y Carreño, 2016:6). Si bien durante los años de juventud suelen experimentarse formas convencionales de entretenimiento y diversión frecuentemente basadas en un espíritu efímero y exclusiva- 
mente comercial, los jóvenes también van más allá del mero divertimento para generar "procesos identitarios, de organización social, formativos" (Cuenca y Carreño, 2016:5) conectados con sus experiencias particulares y con situaciones de la sociedad en la que viven. Los jóvenes se preocupan por su formación y el bienestar de sus contextos y a través de sus experiencias de ocio, vividas individual y colectivamente, se vinculan a proyectos de felicidad de las sociedades (Cuenca y Carreño, 2016:5)

El concepto de ocio se sustenta en tres pilares básicos: la libertad, la gratuidad y la satisfacción. En otras palabras, "Vivir el ocio es ser consciente de la «no obligatoriedad» y de la finalidad no utilitaria de una acción externa o interna, habiendo elegido dicha acción en función de la satisfacción íntima que proporciona" (Cuenca, 2011:60-61). Son estos tres rasgos, determinantes de la calidad subjetiva de la experiencia, los que definen la condición de la experiencia de ocio, y no el momento del tiempo o la naturaleza de la actividad.

Tal y como indica Cuenca (2011), las prácticas de ocio son uno de los indicadores que contribuyen a medir la calidad de vida, dados los múltiples beneficios que de ellas se derivan. Tales beneficios no son meramente individuales, sino también sociales de naturaleza directa (mejora moral de un grupo como consecuencia del incremento de la satisfacción vital de un individuo) o indirecta (reducción del vandalismo) (Tinsley, 2004). Desde nuestro punto de vista, el hecho de emprender se encontraría dentro de este grupo, es decir, tendría beneficios directos sobre el emprendedor, pero también un impacto social importante.

Esta definición responde a las prácticas de ocio en general, sin embargo no todas ellas requieren la misma implicación y compromiso ni reportan los mismos tipos de beneficios. Son varios los autores que diferencian aquellas actividades puntuales, espontáneas y efímeras de las que implican un interés duradero, una preparación, un compromiso y un proceso de crecimiento personal y de desarrollo de habilidades (Kleiber, 2004; Csikszentmihalyi, 2008; Stebbins, 2008; Cuenca, 2011).

Stebbins (2008) crea a finales de los setenta el concepto de ocio serio y algo más tarde el de ocio casual y defiende que, aunque ambos tipos de ocio son necesarios para un estilo de vida de ocio óptimo (Stebbins, 2000), el ocio serio ofrece beneficios que no se encuentran en el ocio casual (Stebbins, 2004). Para este autor el ocio serio representa la "búsqueda sistemática de una actividad amateur, de voluntariado o un hobby, cuya naturaleza le llene al participante y éste la encuentre suficientemente sustancial e interesante como para encontrar en ella una carrera centrada en adquirir y expresar la combinación de habilidades, conocimientos y experiencia específicas de la actividad" (Ste- 
bbins, 2008:xii). Mientras que el ocio casual se refiere a aquella "actividad inmediata e intrínsecamente gratificante, de sensaciones placenteras relativamente efímeras y que requiere poca o ninguna formación específica para disfrutar de ella" (Stebbins, 2008:xii). Según Stebbins (2008), el ocio serio presenta seis características que le son propias: necesidad de perseverar; superación personal; esfuerzo personal significativo que utiliza los conocimientos, formación, experiencia y/o habilidades especialmente adquiridos para llevar a cabo la actividad; beneficios duraderos; carácter comunitario manifestado en actitudes compartidas, prácticas, valores, creencias, metas, etc.; tendencia a una fuerte identificación con la actividad elegida.

Las vivencias de ocio que requieren un mayor compromiso y exigencia constituyen experiencias de ocio valiosas. Sin duda, es imprescindible que el sujeto disfrute de ellas y les atribuya un valor intrínseco (Cuenca Amigo, 2013) y que tales experiencias aporten beneficios para el desarrollo personal (Amigo, 2011).

Llegados a este punto, nos planteamos si este tipo de experiencias de ocio valiosas pueden ser o no un estímulo para emprender. No es la primera vez que se estudia si el ocio puede estar entre las múltiples motivaciones para iniciar un proyecto, puesto que además de los motivos de oportunidad o necesidad puede haber otros muchos, que también evolucionen en el tiempo (Williams y Nadin, 2012). La motivación que origina el emprendimiento es sobre todo un producto del contexto social, económico y espacial en el que los emprendedores se encuentran, así como un resultado del tipo de emprendimiento que está a su alcance. Otro indicador del interés por desentrañar la conexión entre las experiencias de ocio valioso y emprendimiento es la presencia de elementos claves comunes en ambos fenómenos: por un lado, la motivación intrínseca y, por otro, la creatividad y la innovación. Tanto a la hora de emprender como a la hora de vivir un ocio serio y significativo, el desarrollo tiene que ver con la capacidad para innovar, buscando nuevos retos, aumentando para ello la complejidad de la actividad y desarrollando nuevas habilidades. A la luz de estos indicios, este estudio se plantea el objetivo general de explorar el binomio ocio y emprendimiento, lo que se concreta en los siguientes objetivos específicos:

(1) Analizar si existe o no relación entre la probabilidad percibida de que el proyecto Think Big se convierta en empresa y la conexión del proyecto Think Big con cualquier actividad de ocio del encuestado.

(2) Analizar si existe o no relación entre la probabilidad percibida de que el proyecto TB se convierta en empresa y la conexión del proyecto Think Big con la actividad de ocio significativa del encuestado. 
(3) Analizar la relación entre la implicación del encuestado con su actividad significativa de ocio y la conexión del proyecto Think Big con dicha actividad de ocio significativa.

(4) Describir las actividades de ocio significativo que sirven de germen de los proyectos de emprendimiento analizados.

\section{METODOLOGÍA}

Para dar respuesta a los objetivos planteados, se diseñó una metodología de carácter cuantitativo basada en una nueva escala de medición. Los siguientes apartados desarrollan la muestra seleccionada para la intervención, el proceso de construcción de la escala, así como el procedimiento de recogida y análisis de datos.

\section{DESCRIPCIÓN DE PARTICIPANTES}

La población de referencia para la realización de este estudio ha sido el conjunto de jóvenes que participan en el programa Think Big Jóvenes, liderado por Fundación Telefónica en colaboración con Movistar. Se trata de un proyecto de innovación y emprendimiento social que ve la luz en 2012 y que en 2017 celebra su $5^{\text {a }}$ convocatoria. Ofrece a los jóvenes residentes en España de entre 15 y 30 años la oportunidad de transformar una idea propia que busque un cambio positivo en la comunidad en un proyecto de éxito. Las ideas presentadas deben tener un componente digital y beneficiar a su entorno, comunidad o la sociedad. El programa ayuda a los jóvenes a poner dichas ideas en marcha aportándoles conocimientos y herramientas, asesoramiento de profesionales así como una ayuda económica condicionada que se reserva a algunas de las iniciativas. El programa se define como "un proceso de formación continua en habilidades de emprendimiento social que otorga al participante la capacidad de marcar su propio ritmo de crecimiento personal y profesional, a través del desarrollo de un proyecto social y teniendo la oportunidad de pasar por diferentes niveles en función del trabajo, maduración y necesidades de cada proyecto y equipo". Dichos niveles son el 0 (Inspiración), 1 (Start) y 2 (Build). El trabajo y maduración de las ideas es progresivo y el apoyo que brinda la Fundación Telefónica proporcional a los avances logrados. Así, en el nivel más avanzado (2, Build), el apoyo se orienta a la sostenibilidad del proyecto, más allá del Programa Think Big (Fundación Telefónica, 2017).

La muestra de este estudio está compuesta por 65 participantes, 43 proyectos de la fase Start ( $3^{\mathrm{a}}$ convocatoria) y 22 de la fase Build ( $2^{\mathrm{a}}$ convocatoria). No se incluyen participantes del Nivel 0 porque en el momento de realizar el 
trabajo de campo no se ofertaba este nivel. Se trata de una muestra pequeña, no exenta de limitaciones, pero que permite realizar un primer análisis exploratorio.

En relación a la edad, únicamente 18 participantes (28\%) tienen entre 15 y 21 años, mientras que la mayoría se concentra en el intervalo de entre 22 y 26 años (72\%). En el nivel de estudios se observa también un sesgo, en este caso a favor de las personas que cuentan con estudios universitarios (57\%), ya sea de grado (29\%) o postgrado (28\%). El resto de la muestra se reparte entre estudios de secundaria (9\%), bachillerato (26\%) y ciclos formativos (8\%). Las diferencias se acusan aún más si se analizan estos datos en función de la fase en la que se encuentra el proyecto. En este caso, se constata que de los participantes en proyectos de fase Build, el 91\% tienen entre 22 y 26 años y el $82 \%$ cuentan con estudios universitarios ( $32 \%$ grado y $50 \%$ postgrado).

Con respecto al género, el 34\% de la muestra son mujeres y el 66\% hombres. En lo referente a la procedencia de los participantes, todas las Comunidades Autónomas a excepción de La Rioja y Cantabria se encuentran representadas, si bien el 68\% de los individuos procede de las siguientes cuatro comunidades: Madrid (26\%); Comunidad Valenciana (15\%); Cataluña (14\%) y Castilla y León (12\%). El análisis desglosado de proyectos Start y Build no apunta diferencias en las proporciones comentadas de género y procedencia.

Sin embargo, en el caso de la ocupación sí se observan algunas diferencias. Considerando el total del colectivo, un 32\% de los participantes sólo estudia, un $38 \%$ estudia y trabaja, un $17 \%$ trabaja y un $12 \%$ está en paro u otra situación. Entrando en el detalle de los participantes de cada una de las fases, se puede observar que los individuos de la fase Start principalmente sólo estudian (42\%) o estudian y trabajan (37\%), mientras que los de la fase Build fundamentalmente estudian y trabajan (41\%) o trabajan (27\%). Estos datos son coherentes con los datos de ingresos, ya que mientras un $67 \%$ de los participantes en la fase Start viven principalmente de los ingresos de su familia, dicho porcentaje se reduce al 36\% cuando se trata de los participantes Build. No obstante, en la visión de conjunto se puede observar que el colectivo encuestado es bastante dependiente de sus padres, dado que el $71 \%$ vive en el hogar familiar y el $85 \%$ vive gracias a los ingresos de su familia o a alguna ayuda que le proporciona su familia. Cuando se les pregunta por la clase social, la mayoría se situaría en la clase media (52\%), clase media-alta (25\%) o clase media-baja (18\%).

Adicionalmente, es interesante observar que el 63\% de los encuestados tienen algún miembro cercano de su familia que sea o haya sido empresario o tenga un negocio propio. Así mismo, el 85\% de la muestra ha tenido alguna experiencia laboral previa. Por último, un $94 \%$ de quienes responden han par- 
ticipado en la generación inicial de la idea de proyecto y un $75 \%$ asume el rol de líder, por lo que en general su nivel de implicación es elevado. En ninguna de estas variables parece que existan diferencias entre los proyectos en fase Start y Build.

\section{INSTRUMENTO}

El instrumento de medida utilizado ha sido un cuestionario organizado en torno a tres secciones. En la primera se abordaba el tema del emprendimiento y de su participación en Think Big Jóvenes. En el segundo se recogían preguntas relacionadas con sus prácticas de ocio y el modo en que éstas habían inspirado las iniciativas del programa, el tercero recopilaba datos sociodemográficos de los participantes. A continuación se detallan las variables utilizadas en el análisis que aquí se presenta.

En lo referente al bloque de emprendimiento se ha tenido en cuenta: (1) la fase en la que se encontraba el proyecto Think Big (Start o Build); (2) si la persona encuestada participó en la idea inicial del proyecto o no; (3) el deseo de que el proyecto acabara convirtiéndose en su propia empresa y (4) la probabilidad percibida de que el proyecto acabara convirtiéndose en su propia empresa.

En la sección de ocio se preguntaba a los participantes por su actividad de ocio más importante (objetivo 4) y se aplicaba la escala Modified Involvement Scale (MIS) propuesta por Kyle, Absher, Norman, Hammitt y Jodice (2007), y procedente a su vez de la escala defendida por McIntyre (1989), para medir la implicación de la persona con dicha actividad (objetivo 3). La escala consta de 15 ítems de los cuales se han seleccionado los 8 que explicaban mejor las 5 categorías de las que se compone: Atracción, Centralidad, Vinculación social, Afirmación de identidad y Expresión de identidad. La mencionada selección de ítems fue ya utilizada en castellano en el proyecto ITINERE ${ }^{1}$, parte de cuyos resultados han sido presentados por Cuenca-Amigo y San Salvador del Valle (2016) y Cuenca-Amigo, Aristegui Fradua, Cuenca Cabeza y Amigo Fernández de Arroyabe (2017). El alfa de Cronbach para la escala en castellano con 8 ítems y con los datos de la muestra de 65 proyectos de emprendimiento, asciende a 0,813. Tal y como se puede observar en la tabla 1 , la eliminación del quinto ítem correspondiente a la categoría Vinculación Social consigue un aumento del alfa hasta 0,85 . Tras realizar este análisis, dicho ítem se ha descartado y se ha trabajado con una medida agrupada que recogía la media de los datos de la escala teniendo en cuenta únicamente 7 ítems. Nunnally (1987) afirma que el alfa de Cronbach debe ser superior a 0,7, por lo que, dado el

${ }^{1}$ ITINERE: Formación para un ocio experiencial valioso a largo de la vida: contribución de los itinerarios de ocio al envejecimiento satisfactorio. EDU2012-38052. 
resultado obtenido de 0,85 se considera que la escala es fiable. Los participantes respondieron a cada uno de los ítems utilizando un formato de respuesta con puntuaciones comprendidas entre 1 (muy en desacuerdo) y 5 (muy de acuerdo).

El apartado de ocio concluía con dos preguntas claves para poder dar respuesta a todos los objetivos: (1) ¿El proyecto Think Big en el que participas está relacionado con alguna actividad de ocio que tú practicas? (objetivo 1) y (2) ¿El proyecto Think Big en el que participas está relacionado con la actividad de ocio que has marcado como más importante para ti? (objetivos 2, 3 y 4).

Finalmente, los datos sociodemográficos incluidos son los ya mencionados en el apartado de descripción de participantes.

Tabla 1. Ítems seleccionados de la MIS de Kyle et al (2007)

\begin{tabular}{l|l|c|l}
\hline Ítem MIS & Categoría MIS & $\begin{array}{c}\text { Alfa de Cronbach } \\
\text { si el elemento se } \\
\text { ha suprimido }\end{array}$ & Decisión tomada \\
\hline $\begin{array}{l}\text { Esta actividad es muy } \\
\text { importante para mí }\end{array}$ & Atracción & 0,775 & Elemento incluido \\
\hline $\begin{array}{l}\text { Es una de las cosas más } \\
\text { satisfactorias que hago }\end{array}$ & Atracción & 0,775 & Elemento incluido \\
\hline $\begin{array}{l}\text { Ocupa un rol central } \\
\text { en mi vida }\end{array}$ & Centralidad & 0,790 & Elemento incluido \\
\hline $\begin{array}{l}\text { Disfruto hablando de la } \\
\text { actividad con mis } \\
\text { amistades }\end{array}$ & $\begin{array}{l}\text { Vinculación } \\
\text { social }\end{array}$ & 0,777 & Elemento incluido \\
\hline $\begin{array}{l}\text { Muchas de mis amistades } \\
\text { están, de alguna manera, } \\
\text { relacionadas a través de } \\
\text { esta actividad }\end{array}$ & $\begin{array}{l}\text { Vinculación } \\
\text { social }\end{array}$ & 0,850 & Elemento descartado \\
\hline $\begin{array}{l}\text { Cuando practico esta } \\
\text { actividad, puedo } \\
\text { realmente ser yo mismo/a }\end{array}$ & $\begin{array}{l}\text { Afirmación } \\
\text { de identidad }\end{array}$ & 0,791 & Elemento incluido \\
$\begin{array}{l}\text { Practicar esta actividad } \\
\text { dice mucho acerca de } \\
\text { quién soy }\end{array}$ & $\begin{array}{l}\text { Expresión de } \\
\text { identidad }\end{array}$ & 0,779 & Elemento incluido \\
\hline $\begin{array}{l}\text { Buena parte de mi vida } \\
\text { está organizada en torno actividad }\end{array}$ & Centralidad & 0,790 & Elemento incluido \\
\hline
\end{tabular}




\section{PROCEDIMIENTO DE RECOGIDA Y ANÁLISIS DE DATOS}

La recogida de datos se realizó a través de la Fundación Telefónica. Los investigadores diseñaron un cuestionario online construido sobre la plataforma Google Forms y redactaron una carta explicando el objetivo del estudio en la que se incluía el link a la encuesta. Dicha carta fue distribuida por los responsables del programa Think Big a dos colectivos concretos. En primer lugar, durante el mes de julio de 2014, la encuesta se distribuyó entre los participantes de la $2^{a}$ convocatoria de la fase Build, compuesta por 36 proyectos. En esta oleada se obtuvieron 22 respuestas, lo que implica una tasa de respuesta del $61 \%$. En segundo lugar, durante el mes de septiembre de 2014, se hizo llegar la encuesta a los participantes de la $3^{\text {a }}$ convocatoria de la fase Start, compuesta por 330 proyectos. En esta ocasión se obtuvieron 43 respuestas, lo cual equivale a un $13 \%$ de tasa de respuesta. A pesar de que la muestra de sujetos que participaron no se puede considerar representativa del total de jóvenes que participan en el programa Think Big, sí resulta un buen punto de partida para una primera aproximación al objeto de estudio.

Para realizar el análisis de datos, la base de datos en Excel generada automáticamente a través del formulario de Google, fue importada en la aplicación estadística SPSS V.24. Para la búsqueda de relaciones entre variables, se ha aplicado la prueba de la regresión lineal múltiple para obtener un primer acercamiento a los resultados. Posteriormente, para aquellas variables que apuntaban relaciones significativas se ha aplicado también la prueba de la t-Student para contrastar el análisis. En el caso de la escala MIS, se ha trabajado con una medida agrupada de la media de los 7 ítems mencionados en el apartado anterior (Instrumento). Por último, la descripción de actividades se ha realizado teniendo en cuenta tablas de frecuencias.

\section{ANÁLISIS DE RESULTADOS Y DISCUSIÓN}

En primer lugar, para analizar la relación entre ocio y emprendimiento se ha tomado la variable que recoge la probabilidad percibida de que el proyecto Think Big se convierta en empresa. Esta variable se ha definido como dependiente y se han incluido como independientes un total de 18 variables, 10 de las cuales eran datos sociodemográficos y el resto correspondían a las preguntas relacionadas con el ocio, la fase del proyecto y el deseo de que el proyecto se convirtiera en su propia empresa. El modelo presenta un coeficiente de Durbin-Watson de 1,807, que se considera aceptable y un R cuadrado ajustado de 0,204 , que indica que el porcentaje de varianza explicada es muy bajo $(20,4 \%)$. El modelo en su conjunto arroja un grado de significatividad de 0,039, lo cual 
a priori es positivo. Sin embargo, al analizar el detalle del grado de significatividad de cada una de las variables, se puede observar que tan solo tres variables tienen valores menores de 0,05, que son (1) Fase (,047); (2) Deseo de que el proyecto se convirtiera en su propia empresa $(, 005)$ y (3) el proyecto está relacionado o no con la actividad de ocio significativa del encuestado $(, 041)$.

A partir de esta información se ha realizado la prueba de la t-Student para cada una de las tres variables mencionadas, cruzada cada una de ellas con la probabilidad percibida de convertir el proyecto en empresa. Los resultados de la prueba t se pueden consultar en la tabla 2.

A la luz del análisis de resultados de la prueba t, la variable fase no parece guardar una relación significativa con la probabilidad percibida de convertir el proyecto en empresa $(, 070)$ si se mantiene el límite del intervalo en 0,05 . Dada la escasez de datos se podría considerar la ampliación del límite a 0,10 , de tal manera que los datos resultarían coherentes. De forma llamativa, se puede observar que la media de la probabilidad percibida es menor en el caso de los individuos del colectivo Build que en los del colectivo Start. Es

Tabla 2. Prueba t de la probabilidad percibida de convertir el proyecto $\mathrm{TB}$ en empresa con tres variables distintas

\begin{tabular}{|c|c|c|c|c|c|}
\hline Variable dependiente & Variable de agrupación & $\mathrm{N}$ & Media & $\begin{array}{l}\text { Desviación } \\
\text { estándar }\end{array}$ & $\begin{array}{l}\text { Sig. (bilateral) } \\
\text { asumiendo } \\
\text { varianzas } \\
\text { iguales }\end{array}$ \\
\hline \multirow{2}{*}{$\begin{array}{l}\text { Probabilidad percibida } \\
\text { de convertir el } \\
\text { proyecto en empresa }\end{array}$} & Fase: "Start" & 43 & 3,42 & 1,052 & \multirow{2}{*}{, 070} \\
\hline & Fase: "Build" & 22 & 2,91 & 1,065 & \\
\hline \multirow{2}{*}{$\begin{array}{l}\text { Probabilidad percibida } \\
\text { de convertir el } \\
\text { proyecto en empresa }\end{array}$} & $\begin{array}{l}\text { NO desea que el } \\
\text { proyecto se convierta } \\
\text { en empresa }\end{array}$ & 8 & 2,00 & ,756 & \multirow[t]{2}{*}{, 000} \\
\hline & $\begin{array}{l}\text { Sí desea que el } \\
\text { proyecto se convierta } \\
\text { en empresa }\end{array}$ & 57 & 3,42 & ,999 & \\
\hline \multirow{2}{*}{$\begin{array}{l}\text { Probabilidad percibida } \\
\text { de convertir el } \\
\text { proyecto en empresa }\end{array}$} & $\begin{array}{l}\text { Proyecto NO } \\
\text { relacionado con } \\
\text { actividad de ocio } \\
\text { significativa }\end{array}$ & 37 & 2,97 & 1,067 & \multirow{2}{*}{, 017} \\
\hline & $\begin{array}{l}\text { Proyecto relacionado } \\
\text { con actividad de ocio } \\
\text { significativa }\end{array}$ & 28 & 3,61 & ,994 & \\
\hline
\end{tabular}


posible que los participantes con más experiencia sean más conscientes de las dificultades encontradas por el camino que los recién iniciados, quienes quizás sean más ingenuos en este sentido.

Sin embargo, en el caso de las otras dos variables no surgen dudas, ya que el resultado de la prueba t concuerda con lo obtenido en la regresión. En primer lugar, se puede observar que el deseo de que el proyecto se convierta en empresa está muy relacionado con la probabilidad percibida de que eso ocurra $(, 000)$, lo cual tiene sentido. En segundo lugar, también se confirma de nuevo la relevancia de que el proyecto esté relacionado con una actividad de ocio significativa del participante. Cabe resaltar que no es suficiente que el proyecto esté relacionado con cualquier actividad de ocio, sino que dicha actividad de ocio debe ser la actividad de ocio significativa, es decir, la más importante para el individuo. En el caso de tratarse de cualquier actividad de ocio, la diferencia entre medias no es significativa (,174). Este hallazgo apoya el pensamiento expuesto por Cuenca y Carreño (2016) de que de ocio más trascendente y exigente puede constituir un modo de implicarse en proyectos de mejora colectiva para la juventud.

Para profundizar en las actividades de ocio significativas se ha utilizado la escala MIS para medir la implicación de cada participante con la mencionada actividad. En este caso, de nuevo se ha aplicado la prueba t considerando la variable agrupada de la escala MIS (media de los 7 ítems escogidos) y si el proyecto estaba o no relacionado con la actividad de ocio significativa. El análisis de medias muestra, efectivamente, que el nivel de implicación es más alto para aquellos participantes cuyo proyecto está relacionado con su actividad de ocio significativa $(4,3163)$ que para los que no lo está $(4,0541)$. Sin embargo, el grado de significatividad se encuentra de nuevo en el límite (,104), probablemente debido a la escasez de datos. A pesar de ello, los resultados son coherentes con la tesis de que quienes se embarcan en la aventura de poner en marcha proyectos de esta índole demuestran altos niveles de motivación intrínseca y de creatividad (Amabile, 1996; Weitzel et al, 2010), factores que también son determinantes para que una práctica de ocio sea seria y significativa (Stebbins, 2008). También reafirman la idea sostenida por Amabile (1996, 1997) sobre la importancia de la creatividad emprendedora y su relación indisociable con una alta motivación intrínseca.

Por último, para entender mejor la naturaleza de las actividades de ocio significativas, resulta de interés conocer de qué tipo de actividades se trata y agruparlas atendiendo a las cinco dimensiones fundamentales de ocio propuestas por Cuenca (2004): lúdica, creativa, ambiental-ecológica, festiva y la solidaria. Añadimos una sexta, la dimensión social, cuya finalidad sería socializar y compartir con amistades el tiempo de ocio. La tabla 3 recoge las actividades de ocio y sus categorías tanto para el total de individuos encuestados $(n=65)$ como 
para aquellos cuyos proyectos están relacionados con prácticas de ocio significativas $(n=28)$.

Tabla 3. Actividades de ocio marcadas como significativas $(n=65)$ y actividades de ocio marcadas como significativas y además relacionadas con proyectos de emprendimiento $(n=28)$

\begin{tabular}{|c|c|c|c|}
\hline Categoría & $\begin{array}{l}\text { Actividad de ocio significativa } \\
\text { para el encuestado }\end{array}$ & $\begin{array}{c}\text { Total de } \\
\text { encuestados }\end{array}$ & $\begin{array}{l}\text { Encuestados cuyo } \\
\text { proyecto está } \\
\text { relacionado con } \\
\text { actividad de ocio }\end{array}$ \\
\hline \multirow{8}{*}{ Creativa } & $\begin{array}{l}\text { Actividades físicas artísticas y } \\
\text { expresivas (Bailes, gimnasia } \\
\text { artística, etc.) }\end{array}$ & 1 & 0 \\
\hline & $\begin{array}{l}\text { Buscar información concreta } \\
\text { en internet }\end{array}$ & 2 & 2 \\
\hline & $\begin{array}{l}\text { Escribir mi propio blog } \\
\text { o página web }\end{array}$ & 3 & 3 \\
\hline & $\begin{array}{l}\text { Hacer alguna actividad artística, } \\
\text { musical o literaria (tocar un } \\
\text { instrumento, pintar, escribir, etc.) }\end{array}$ & 10 & 5 \\
\hline & $\begin{array}{l}\text { Hacer manualidades (bricolaje, } \\
\text { cerámica, etc.) }\end{array}$ & 1 & 0 \\
\hline & $\begin{array}{l}\text { Ir a museos, galerías o } \\
\text { exposiciones }\end{array}$ & 1 & 0 \\
\hline & Leer libros que me gustan & 3 & 1 \\
\hline & Ver películas en el cine o en casa & 1 & 0 \\
\hline \multirow[t]{2}{*}{ Subtotal di } & ión creativa & 22 & 11 \\
\hline & $\%$ & $34 \%$ & $39 \%$ \\
\hline \multirow{4}{*}{ Ecológica } & $\begin{array}{l}\text { Actividades físicas en el medio } \\
\text { natural (senderismo, escalada, } \\
\text { excursiones a pie o en bicicleta, etc.) }\end{array}$ & 3 & 1 \\
\hline & $\begin{array}{l}\text { Hacer excursiones al campo, } \\
\text { a la montaña o a la playa }\end{array}$ & 1 & 1 \\
\hline & Turismo & 3 & 2 \\
\hline & Viajar & 7 & 4 \\
\hline \multirow[t]{2}{*}{ Subtotal dimensión ecológica } & ión ecológica & 14 & 8 \\
\hline & $\%$ & $22 \%$ & $29 \%$ \\
\hline
\end{tabular}




\begin{tabular}{|c|c|c|c|c|}
\hline Categoría & \multicolumn{2}{|c|}{$\begin{array}{l}\text { Actividad de ocio significativa } \\
\text { para el encuestado }\end{array}$} & $\begin{array}{c}\text { Total de } \\
\text { encuestados }\end{array}$ & $\begin{array}{l}\text { Encuestados cuyo } \\
\text { proyecto está } \\
\text { relacionado con } \\
\text { actividad de ocio }\end{array}$ \\
\hline \multirow{3}{*}{ Lúdica } & \multicolumn{2}{|c|}{$\begin{array}{l}\text { Actividades de oposición uno } \\
\text { contra uno (actividades de lucha, } \\
\text { tenis, etc.) }\end{array}$} & 1 & 0 \\
\hline & \multicolumn{2}{|c|}{$\begin{array}{l}\text { Actividades físicas de } \\
\text { cooperación y oposición } \\
\text { (baloncesto, voleibol, fútbol, etc.) }\end{array}$} & 2 & 0 \\
\hline & \multicolumn{2}{|c|}{$\begin{array}{l}\text { Actividades individuales } \\
\text { (correr, pasear, patinaje, } \\
\text { natación, etc.) }\end{array}$} & 1 & 0 \\
\hline \multirow{2}{*}{\multicolumn{2}{|c|}{ Subtotal dimensión lúdica }} & $N$ & 4 & 0 \\
\hline & & $\%$ & $6 \%$ & $0 \%$ \\
\hline Social & \multicolumn{2}{|c|}{$\begin{array}{l}\text { Salir a tomar algo con las } \\
\text { amistades }\end{array}$} & 11 & 1 \\
\hline \multirow{2}{*}{\multicolumn{2}{|c|}{ Subtotal dimensión social }} & $N$ & 11 & 1 \\
\hline & & $\%$ & $17 \%$ & $4 \%$ \\
\hline \multirow{3}{*}{ Solidaria } & \multicolumn{2}{|c|}{ Colaborar con mi asociación } & 8 & 5 \\
\hline & \multicolumn{2}{|c|}{ Cuidar animales } & 2 & 1 \\
\hline & \multicolumn{2}{|c|}{ Hacer voluntariado (ONGs, etc.) } & 4 & 2 \\
\hline \multirow{2}{*}{\multicolumn{2}{|c|}{ Subtotal dimensión solidaria }} & $N$ & 14 & 8 \\
\hline & & $\%$ & $22 \%$ & $28 \%$ \\
\hline \multicolumn{3}{|c|}{ Total general } & 65 & 28 \\
\hline
\end{tabular}

La tabla 3 muestra que, para el conjunto de la muestra, las actividades significativas están relacionadas fundamentalmente con la dimensión creativa, seguido de las dimensiones solidaria, ecológica y social. En concreto, hay dos ítems que destacan: "Salir a tomar algo con las amistades" (dimensión social) y "Hacer alguna actividad artística, musical o literaria: tocar un instrumento, pintar, escribir, etc." (Dimensión creativa).

Realizando el mismo ejercicio con las actividades significativas que además están relacionadas con proyectos de emprendimiento, los datos cambian. Tomando en consideración únicamente los proyectos basados en la actividad de ocio significativa resulta que las dimensiones creativa, solidaria y ecológica 
aumentan su porcentaje, mientras que la social se reduce y la lúdica desaparece. Por tanto, parece que no cualquier actividad de ocio significativa se presta a convertirse en un proyecto de emprendimiento. Sin embargo, los datos disponibles no permiten afirmar que exista una relación entre el tipo de actividad de ocio y el hecho de que un proyecto esté o no relacionado con una actividad de ocio (prueba de regresión con Sig. >0,05). A pesar de ello, resulta coherente que quienes dan más importancia a las prácticas de ocio solidario realicen proyectos de emprendimiento social, cuya esencia consiste en transformar el mundo en un lugar mejor. Y también apoya la relación positiva existente entre la práctica de actividades culturales entre los jóvenes y su propensión a participar en proyectos sociales (Rivera y Santos, 2016).

\section{CONCLUSIONES}

La revisión teórica que hemos llevado a cabo nos conduce a confirmar ciertas ideas. La crisis que aún perdura en España tiene un impacto directo en los jóvenes, quienes ven mermadas sus posibilidades de desarrollo personal e integración en la vida social. Hoy, más que nunca, se necesitan medidas impulsoras del concepto de ciudadanía juvenil, que considera a los jóvenes protagonistas de su condición ciudadana y que aboga por la conquista de espacios de autonomía y de participación (Benedicto, 2016b). El emprendimiento, y en especial el emprendimiento social, es una de las posibles vías para propiciar la ocupación de espacios individuales y comunitarios por los jóvenes. En línea con quienes conciben el emprendimiento más allá de un fenómeno meramente económico, para los jóvenes emprender puede llegar a ser una actitud frente a la vida y, probablemente, para toda la vida. Un camino que les permita aprovechar las oportunidades que la crisis les brinde. Esta actitud responde a la emergente demanda social de capacidades emprendedoras que aporten innovación y que desemboquen en una sociedad más participativa y democrática. Además, el emprendimiento social es un cauce en auge para poner en marcha iniciativas innovadoras que transformen la sociedad.

Asimismo, la lectura de los datos obtenidos nos permite extraer ciertas conclusiones. En primer lugar, hemos probado que aquellos participantes cuyo proyecto Think Big estaba relacionado su actividad de ocio significativa, percibían una probabilidad más alta de que su proyecto se convirtiera en su propia empresa (objetivo 2) que el resto. Sin embargo, esto no ocurría con cualquier actividad de ocio (objetivo 1), sino únicamente con la actividad de ocio significativa. Esto resulta de gran interés y como ya se ha expuesto anteriormente es coherente con las teorías procedentes de los estudios de ocio, como por ejemplo, toda la teoría de ocio serio de Stebbins (2008). En concreto, este autor 
defiende (Stebbins, 2010) que al analizar el emprendimiento social desde la perspectiva de ocio serio podemos enriquecer nuestro entendimiento del fenómeno. Así, esta óptica enfatiza la búsqueda de recompensas personales y sociales del individuo, así como la vivencia de la propia experiencia en sí. Para Stebbins (2010), el emprendimiento social es una práctica de ocio serio y no casual.

De forma coherente con todo lo anterior, también hemos podido comprobar que aquellos cuyo proyecto Think Big estaba relacionado su actividad de ocio significativa demostraban una mayor implicación con su actividad de ocio significativa que el resto. De alguna manera, una actividad de ocio significativa que se convierte en proyecto de emprendimiento pasa a convertirse en un trabajo. A priori, esto puede resultar contradictorio, pero tal y como defiende Stebbins (2010), las personas pueden experimentar flujo en el trabajo y es, precisamente, en estos casos cuando la frontera entre el trabajo y el ocio se desdibuja.

Por último, al analizar las prácticas de ocio significativo atendiendo a las dimensiones del ocio intuimos que cuando la actividad significativa pertenece a la esfera creativa y solidaria, es cuando parece que pueden existir más probabilidades de que dé lugar a un proyecto de emprendimiento social. A pesar de que los datos apuntan esta tendencia, la relación entre variables no ha podido ser confirmada en la presente investigación, probablemente debido a las limitaciones de la muestra. Sin embargo, consideramos que esta línea de investigación podría ser explorada en profundidad a futuro, dado que existe literatura que la apoya (Rivera y Santos, 2016).

Los resultados del análisis de datos pueden derivar tanto en líneas de intervención como de investigación. Por un lado, en lo referente a la práctica, puede resultar de interés para programas de emprendimiento social, como Think Big, la búsqueda de contacto y alianzas con clubs o asociaciones de aficionados o voluntarios, ya que estos colectivos son claros practicantes de ocio serio. La difusión del programa de emprendimiento entre estos colectivos quizás podría derivar en un mayor número de proyectos que se convirtieran en empresas. Por otro lado, y teniendo en cuenta las limitaciones derivadas del tamaño de la muestra ya mencionadas, este estudio exploratorio abre la puerta a futuras investigaciones que, por una parte, corroboren la mayor implicación de quienes ponen en marcha un proyecto social conectado con sus prácticas de ocio significativas y, por otra, analicen la relación entre los distintos tipos de actividades de ocio y su papel central en proyectos de emprendimiento social.

Para finalizar, resaltamos el papel beneficioso que iniciativas como Think Big Jóvenes pueden tener, especialmente para los jóvenes que desarrollan un 
proyecto de emprendimiento social basado en una actividad significativa de ocio, pues además de los beneficios sociales de su emprendimiento, las vivencias que atesoren durante estos años, pasarán a formar parte de su capital de ocio durante el resto de sus vidas.

\section{BIBLIOGRAFÍA ACTUALIZADA}

Aceleanu, M. (2013). The labour market in the post-crisis economy: The case of Spain. Theoretical and Applied Economics: GAER Review, 20(3), 135-146.

Alcañiz, M. y Querol, V. A. (2015). Jóvenes y trayectorias a la vida adulta. Desigualdades, retos y nuevas formas en un contexto de crisis. Recerca: revista de pensament $i$ anàlisi, (16), 7-12.

Alonso, D., González, N. y Nieto, M. (2015): Emprendimiento social vs innovación social. Cuadernos Aragoneses de Economía, vol. 24, nº 1-2, (119-140).

Amabile, T. M. (1996) Creativity in context: Update lo the social psychology of creativity. Boulder. CO: Westview Press.

Amabile, T. M. (1997). Entrepreneurial Creativity through Motivational Synergy. Journal of Creative Behavior, 31(1), 18-26.

Benedicto, J. (2016a). ¿Una generación marcada por la crisis? En J. Benedicto. (Dir.), Informe Juventud en España 2016 (pp. 15-33). Madrid, España: @ Instituto de la Juventud. Disponible en: http://www.injuve.es/observatorio/demografia-e-informacion-general/informe-juventud-en-espana-2016 (última consulta: 16 de agosto de 2017).

Benedicto, J. (2016b). La ciudadanía juvenil: Un enfoque basado en las experiencias vitales de los jóvenes/Youth citizenship: An approach based on the life experiences of young people/A cidadania juvenil: Um enfoque com base nas experiências vitais dos jovens. Revista Latinoamericana de Ciencias Sociales, Niñez y Juventud, 14(2), 925-938. doi:http://0-dx.doi.org.cataleg.uoc.edu/10.11600/16927 $15 x .14203210415$

Bosma, N., Schøtt, T., Terjesen, S. y Kew, P. (2016) GEM 2015 Report on Social Entrepreneurship. Obtenido de: http://gemconsortium.org/report/49542 (última consulta 17 marzo 2017)

Csikszentmihalyi, M, (1975). Beyond boredom and anxiety. San Francisco: Jossey-Bass.

Csikszentmihalyi, M. (2008). Fluir. Una psicología de la felicidad. Barcelona: Kairós.

Cuenca-Amigo, M. y San Salvador del Valle, Roberto. (2016). La importancia del ocio como base para un envejecimiento activo y satisfactorio. Revista de Psicología del Deporte,25(Suppl 2), 79-84.

Cuenca-Amigo, M., Aristegui Fradua, I., Cuenca Cabeza, M. y Amigo Fernández de Arroyabe, M. L. (2017). The importance of leisure in older adults living in Spain. Annals of Leisure Research,20(2), 222-239.

Cuenca Amigo, J. (2013). El valor de la experiencia de ocio en la modernidad tardía. Bilbao: Universidad de Deusto. 
Cuenca, M. (2004). Pedagogía del ocio: Modelos y Propuestas. Bilbao: Publicaciones Universidad de Deusto.

Cuenca, M. (2011). La fuerza transformadora del ocio. En M. Cuenca Cabeza, E. Aguilar Gutiérrez y C. Ortega (Eds.), Ocio para innovar (pp. 17-82). Bilbao: Universidad de Deusto.

Cuenca, M. y Carreño, J.M. (2016). Editorial: Ocio y jóvenes. Lúdica pedagógica. No 23 (2016 - I) pp. 05-06

European Commission. DG Regional and Urban Policy (2013). Guide to social innovation. Obtenido de: http://ec.europa.eu/regional_policy/sources/docgener/presen ta/social_innovation/social_innovation_2013.pdf (Última consulta 15 de marzo de 2017)

Fundación Telefónica (s.f.). www.thinkbigjovenes.fundaciontelefonica.com (Última consulta: 13 de marzo de 2017)

Gentile, A. (2015). Jóvenes titulados superiores en la encrucijada de la crisis. Recerca: Revista De Pensament I Anàlisi, (16), 35-58.

Guzmán Vásquez, A. y Trujillo Dávila, M. (2008). Emprendimiento social-revisión de literatura. Estudios Gerenciales, 24(109), 105-125.

Hennessey, B. (2015). Creative Behavior, Motivation, Environment and Culture: The Building of a Systems Model. Journal of Creative Behavior, 49(3), 194-210.

Hernández, M. J. Parra G. y Pérez, M. D. (2016). Priorización de valores en estudiantes jóvenes y mayores en el contexto de la crisis económica. Teoría de la Educación. Revista Interuniversitaria, 28 1, 105-207.

INJUVE (2016). Jóvenes en la EPA. Cuarto Trimestre 2016. Obtenido de: http://www. injuve.es/observatorio/formacion-empleo-y-vivienda/jovenes-en-la-epa-cuartotrimestre-2016 (Última consulta: 13 de Marzo de 2017)

Kyle, G., Absher, J., Norman, W., Hammitt, W., y Jodice, L. (2007). A modified involvement scale. Leisure Studies, 26(4), 399-427.

Light, P. (2009), Reorganización del emprendimiento social. Revista Espanola del Tercer Sector, 13, 89-97.

McIntyre, N. (1989) The personal meaning of participation: enduring involvement, Journal of Leisure Research 21, pp. 167-179.

Morales Gutiérrez, A., y Montes, J. (2013). Valores, actitudes y motivaciones en la juventud ante el emprendimiento individual y colectivo. Revesco: Revista de Estudios Cooperativos, (112), 11-35.

Neck, H., Brush, C., y Allen, E. (2009). The landscape of social entrepreneurship. Business horizons, 52(1), 13-19.

Nunnally, J. C. (1987).Teoría Psicométrica.México: Trillas.

Rivera, C. (2011). ¿De qué hablamos cuando hablamos de entrepreneurship? Cuadernos Unimetanos 26, 11-12. Obtenido de: http://www.unimet.edu.ve/unimetsite/wp-con tent/uploads/2013/02/CU_V26.pdf (Última consulta: 1 de marzo de 2017).

Rivera, R., y Santos, D. (2015). El perfil de los futuros emprendedores sociales: Competencias y estilos de vida. Revista de Estudios de Juventud, 107, 13-28. 
Rivera, R., y Santos, D. (2016). Civic and Political Participation of Children and Adolescents: A Lifestyle Analysis for Positive Youth Developmental Programs. Children E Society, 30(1), 59-70.

Roberts, K. (2012). El ocio de los jóvenes en la sociedad contemporánea. Arbor, [S.l.], v. 188, n. 754 , p. 327-337, Apr. 2012. ISSN 1988-303X.

Schwagler, N. (2014). Integrating Creative Thinking Skills Into Entrepreneurship Curricula: A Methods Approach. In ICSB World Conference Proceedings (p. 1). International Council for Small Business (ICSB). Disponible en: https://searchproquest-com.proxy-oceano.deusto.es/docview/1796775246? accountid=14529 (Última consulta: 1 de Marzo de 2017).

Stebbins, R. A. (2000). Optimal leisure lifestyle: Combining serious and casual leisure for personal well-being. En M. Cuenca Cabeza (Ed.), Leisure and human development: Proposals for the 6th World Leisure Congress (pp. 101-107). Bilbao: University of Deusto.

Stebbins, R. A. (2004). Ocio serio: $;$ debemos fomentarlo? ADOZ. Boletín del Centro de Documentación en Ocio - Universidad de Deusto, 28, 47-50.

Stebbins, R. A. (2008). Serious leisure: a perspective for our time. New Brunswick: Transaction.

Stebbins, R. A. (2010). Social Entrepreneurship as Work and Leisure.LSA Newsletter, 85, 30-33. Obtenido de http://www.seriousleisure.net/uploads/8/3/3/8/8338986/ reflections23.pdf (última consulta: 8 de septiembre de 2017).

Timmons, J. S. (2009). New Venture Creation: Entrepreneurship for the 21st Century. Boston: McGraw Hill/Irwin.

Tinsley, H. E. A. (2004). Los beneficios del ocio. ADOZ. Boletín del Centro de Documentación en Ocio - Universidad de Deusto, 28, 55-58.

Weitzel, U., Urbig, D., Desai, S., Sanders, M., y Acs, Z. (2010). The good, the bad, and the talented: Entrepreneurial talent and selfish behavior. Journal of Economic Behavior and Organization, 76 (1), 64-81. doi:10.1016/j.jebo.2010.02.013 (Última consulta: 16 de Agosto de 2017).

Williams, C. C., y Nadin, S. (2012). Beyond the commercial versus social entrepreneurship dichotomy: A case study of informal entrepreneurs. Journal of Developmental Entrepreneurship, 17(3), 1.

AMAIA MAKUA BIURRUN: Universidad de Deusto, Estudios de Ocio. Avenida de las Universidades, 24, 480007 Bilbao, España. Tel. 944139000. E-mail. amaia.makua@deusto.es. Código ORCID: orcid.org/0000-0002-8511-8950 Doctora en Ocio y Desarrollo Humano. Desarrolla su labor investigadora en el Equipo de Investigación del Instituto de Estudios de Ocio de la Universidad de Deusto. Es autora de artículos en diversas publicaciones como: Academia Revista Latinoamericana de Administración, Revista de Psicología del Deporte, 
International Journal of Religious Tourism \& Pilgrimage, LSA Publication, ROTUR: Revista de Ocio y Turismo o Revista Turismo \& Desenvolvimento.

MACARENA CUENCA-AMIGO: Universidad de Deusto, Deusto Business School. Hermanos Aguirre 2, 48014 Bilbao, España. Tel. 944139000. E-mail. macarena.cuenca@deusto.es. Código ORCID: orcid.org/0000-0002-8629-7364 Doctora en Ocio y Desarrollo Humano. Profesora en Deusto Business School y miembro del Equipo de Investigación del Instituto de Estudios de Ocio de la Universidad de Deusto. Ha publicado en revistas, tales como: Academia Revista Latinoamericana de Administración, Annals of Leisure Research, Arbor, Cuadernos de Gestión, International Review of Social Research, Revista de Psicología del Deporte.

ROBERTO SAN SALVADOR DEL VALLE DOISTUA: Es Licenciado en Filosofía y Letras (Historia), Doctor en Filosofía y Ciencias de la Educación y Master en Ocio y Potencial Humano por la Universidad de Deusto y Leeds University. Actualmente es Catedrático de la Facultad de Ciencias Sociales y Humanas en la Universidad de Deusto, donde imparte docencia en los campus de Bilbao y Donostia-San Sebastián. Es Director de Deusto Cities Lab Katedra. Ha sido Director del Instituto de Estudios de Ocio (2000-2007) y Vicerrector de Comunicación, Plurilingüismo e Innovación Social (2007-2015). Sus publicaciones, artículos y libros tratan principalmente sobre el fenómeno del ocio, ciudad y políticas. En los últimos años ha estado trabajando en proyectos competitivos y concertados relacionados con la gobernanza e innovación social. Otras áreas de interés investigador son las cuestiones relativas a tendencias sociales, la transformación de las ciudades, y la evolución de los conceptos tiempo y espacio. Pertenece al equipo de investigación Ocio y Desarrollo Humano, que cuenta con el reconocimiento oficial del Gobierno Vasco. Ha sido Chairperson de la World Leisure Policies Commission. Es miembro del Consejo del Basque Social Innovation, Consejo Cívico de Bilbao, Consejo de Bilbao Bizkaia Design Creative Council. Su actividad docente se concentra en el Grado de Turismo, Master Universitario en Dirección de Proyectos de Ocio, Cultura, Turismo, Deporte y Recreación y Master Universitario en Organización de Congresos, Eventos y Ferias, así como en el Doctorado en Ocio y Desarrollo Humano.

Recibido: 06/04/2017

Aceptado: 16/09/2017 


\title{
LOS BENEFICIOS DEL OCIO JUVENIL Y SU CONTRIBUCIÓN AL DESARROLLO HUMANO ${ }^{1}$ THE BENEFITS OF YOUTH LEISURE AND THEIR CONTRIBUTION TO HUMAN DEVELOPMENT
}

\author{
María Jesús Monteagudo \\ Instituto de Estudios de Ocio \\ Facultad de Ciencias Sociales y Humanas \\ Universidad de Deusto, España \\ mjmonte@deusto.es \\ Ruth Ahedo \\ Instituto de Estudios de Ocio \\ Facultad de Ciencias Sociales y Humanas \\ Universidad de Deusto, España \\ ruth.ahedo@deusto.es \\ Ana Ponce de León \\ Grupo AFYDO \\ Facultad de Letras y de la Educación \\ Universidad de la Rioja, España \\ ana.ponce@unirioja.es
}

\begin{abstract}
Cómo citar / Citation
Monteagudo, María Jesús; Ahedo, Ruth y Ponce de León, Ana (2017) "Los beneficios del ocio juvenil y su contribución al desarrollo humano". OBETS. Revista de Ciencias Sociales, 12(Extra 1): pp-pp. 177-202.
\end{abstract}

doi:10.14198/OBETS2017.12.1.17

\footnotetext{
${ }^{1}$ Esta investigación forma parte del Proyecto "De los tiempos educativos a los tiempos sociales: la participación de los jóvenes en la creación y gobernanza de sus espacios de ocio como factor de desarrollo personal" (RESORTES), financiada en la convocatoria del Plan Nacional de I+D+i (2012), por la Secretaría de Estado de Investigación, Desarrollo e Innovación, adscrita al Ministerio de Economía y Competitividad, con referencia EDU201239080-C07-03.
} 


\title{
Resumen
}

Este artículo analiza los beneficios del ocio juvenil y su aportación al desarrollo personal y social con el objetivo de esclarecer si el tipo de actividad de ocio, el género, los espacios y el carácter espontáneo u organizado del ocio inciden en la percepción de beneficios emocionales, cognitivos, sociales, físicos y desarrollo de destrezas y habilidades técnicas. La muestra se compone de 1253 jóvenes españoles con edades entre los 16 y 18 años. Los resultados corroboran el influjo de estas variables tanto en la percepción de beneficios como en la naturaleza de los mismos. El ocio físico-deportivo no es solo la práctica más significativa, sino también la que les proporciona un abanico más amplio de retornos positivos. Los chicos obtienen puntuaciones más altas en todos los tipos de beneficios, excepto en los emocionales. Algunos espacios del ocio resultan más proclives para el logro de ciertos beneficios. Finalmente, el ocio organizado aporta mayores beneficios que el no organizado. La discusión sobre el rol de estas variables es clave para hacer del ocio un factor de desarrollo juvenil.

Palabras clave: Ocio joven, Beneficios, Tipo de Práctica, Género, Espacios, Organización del ocio.

\begin{abstract}
This study aims to explore the benefits of youth leisure in order to clarify whether the type of leisure activity, gender, space and organization of leisure practice affect the perception of different categories of benefits: emotional, cognitive, social physical and the acquisition of abilities and technical skills. The sample consists of 1253 young Spaniards aged between 16 and 18 years. The results confirm the influence of these variables both in the perception of benefits and nature thereof. Physical-sport leisure is not only the most significant practice, but also the one that gives them a wider range of positive returns. The boys score higher in all types of benefits, except for the emotional. Some spaces of leisure are more prone to the achievement of certain benefits. Finally, organized leisure brings greater benefits than non-organized. The discussion on the role of these variables is key to making leisure a factor of youth development.
\end{abstract}

Key words: youth leisure; benefits; type of practice; gender; space; organization of leisure.

\section{Extended abstract}

This work is part of the coordinated project "From educational time to social time: building the human condition of youth in network societies", whose universe is the young Spanish population aged between 16 and 18 years. The study delves into the benefits of youth leisure, focusing on those leisure practices that are identified as particularly significant, that is, those that are especially important for them, and which they do not want to give up. The study is addressed from the approach of young leisure as a valuable experience, which provides a new perspective to look into the benefits of leisure.

Understanding youth leisure in an experiential way implies recognizing that not all experiences of youth leisure contribute identically to personal and 
social development (Cuenca, 2015, Monteagudo, 2008). The concept of valuable leisure (Cuenca, 2015, Kleiber, Bayón, Cuenca and Monteagudo, 2014), coined by the Institute of Leisure Studies of the University of Deusto, focuses attention on the study of leisure experiences that, being highly significant, manage to transform or improve in somehow its protagonist. Thus, the potential of leisure is highlighted to boost individual and/or collective human development processes through the multiple benefits that it offers (Cuenca, 2015; Kleiber, Walker and Mannell, 2011; Nussbaum, 2012; Porter, Iwasaki and Shank, 2010, Valdemoros, Ponce de León and Gradaille, 2016). A representative sample of 1253 young people (48\% girls and 52\% boys) attending post-compulsory secondary education in public and private centers in Spain completed an ad hoc questionnaire structured in 7 large blocks, such as, leisure and free time, family and school life, health and quality of life, the labor market and entrepreneurship, as well as sociodemographic variables. The sample is representative with an error of $2.3 \%$ and a confidence level of $95 \%$. A quantitative methodological design and simple random sampling have been used. The sections that have been analyzed for this article are those dedicated to leisure and spare time, mainly focusing on the following variables: most significant leisure activities (independent variable), leisure benefits (dependent variable), the organization of the activities, spontaneous, organized or both (independent variable), and spaces for the most significant juvenile leisure (independent variable).

There are three categories of benefits that young Spanish population obtains from their significant leisure: emotional, social and cognitive. For this group, the most significant leisure is an inexhaustible source of emotional benefits. These benefits, associated with the satisfaction of doing what they really like, reveal the intrinsic orientation of these young people towards their leisure, therefore, this practice constitutes a benefit in itself. The emotional benefits have higher scores and their importance does not vary with the variables analyzed. The most significant youth leisure also provides a high dose of social and cognitive benefits.

The most important leisure activities are, in order of significance, physical and sport leisure, cultural activities and parties and celebrations. The physical-sport activities are those that report a greater perception of benefits, mainly physical, emotional, as well as the acquisition of skills and technical skills. Physical-sport leisure is not only the most significant practice, but also the one that gives them a wider range of positive returns. Festivals and celebrations, the third type of preferred activity, provide them with emotional and social benefits. It also draws the attention that cultural activities, chosen by the youth group as one of their priority leisure activities, are a more moderate source of benefits than other less significant activities. Cultural leisure provides, above all, emotional and cognitive benefits, but to a lesser extent than practices such as tourism and hiking or volunteering and associationism. Activities that are a priority for a lower percentage of young people, and yet, for the small percentage who practice them, they are a source of significant emotional, cognitive and especially social benefits. In this sense, it should also be noted that, 
despite the rise of digital leisure among young people, this does play an important role among their most significant practices. Recent studies on digital leisure suggest similar findings (Viñals and Cuenca, 2016).

In all types of benefit, women show lower levels of perception than boys. In addition, the types of benefits perceived by boys and girls are different. The girls claim to obtain technical skills and abilities from their leisure but to a lesser extent than the boys. The same goes for physical benefits, both strongly associated with physical-sport leisure. However, mean scores equated when it comes to emotional benefits, which means that both, boys and girls enjoy a highly satisfying leisure. These results point out the existence of important differences regarding the leisure time of girls and boys (what they find most significant, the time devoted, the frequency or company which they do it with, etc.). However, according to Henderson (2009) it can be concluded that in order to advance the understanding of the role of gender in youthful leisure it is necessary to overcome the usual treatment of this aspect (gender) as an independent variable and to deepen in the factors that are involved in the social construction of gender from more neutral approach.

The results confirm that certain spaces ease the achievement of some types of benefits, in line with the activities they host. Thus, open public spaces congregate, especially, the physical-sport leisure, the popular festivals and being with the friendships. All this explains why this type of space is closely associated with emotional and social benefits. Other closed spaces, such as sports centers, gyms, cinemas, play centers, cultural centers, discotheques or bars, host almost half of the physical and sport leisure activities of young people, as well as a large part of their cultural activities. Hence, in addition to high levels of emotional and social benefits, these spaces are associated with the perception of physical benefits and the acquisition of technical skills and abilities. Finally, private spaces (house, private facilities or markets), which host a large number of youth cultural activities and, to a lesser extent, private parties or celebrations, seem to be the main source of emotional and social benefits. Literature emphasizes that young people prefer and feel more satisfied in leisure spaces that provide them some autonomy. The results of this study reach the same conclusion that the previous ones (Abbot-Champan and Robertson, 2001) and point out that the satisfaction (emotional benefits) of youth is high in all the spaces, not only in private ones (a priori, especially prone to selfmanagement). It also confirms that being satisfied with the particular closed spaces does not necessarily imply that high levels of other categories of benefits are obtained in them. In fact, this study confirms that the greater perception of benefits occurs in the closed non-own spaces, in which leisure activities are organized, which are precisely the type of leisure that emerges as the main source of benefit.

Although more than half of the activities of leisure especially significant for Spanish youth are not held by any entity ( 6 out of 10 ), the results show that the perception of benefits is greater when the activity is performed in an organized framework. This difference in terms of benefits in favor of organized leisure explains why the physical-sport activities and associationism, which are 
most frequently performed in an organized framework, are also those that provide higher levels of benefits. Furthermore, digital leisure and cultural activities, which is performed to a greater extent in an unorganized way, are those that show lower mean scores in all categories of benefits, apart from the emotional ones. These results coincide with those obtained in previous studies (Codina, Pestana, Castillo and Balaguer, 2016, Parra, Oliva and Antolín, 2009, Ramos, Rivera and Moreno, 2012), which confirm that organized leisure encourages youth due to the many benefits it provides. The scientific literature confirms the interest of the youth to engage in organized leisure practices, thereby facilitating a greater achievement of different kinds of benefits. However, these data must be interpreted with caution, bearing in mind that the research about this topic is focused to organized leisure.

There is no evidence of studies that have reviewed the positive returns of unorganized youth leisure. In this article it is the young people themselves who announce that organized leisure is more fruitful, in terms of benefits, than the one they realize on their own. However, far from discarding the benefits of unorganized leisure in the face of evidence of data, we reaffirm the need to continue studying unorganized leisure to clarify the nature or conditions in which the contribution of unorganized leisure to development is optimized youth.

\section{INTRODUCCIÓN}

Entender el ocio juvenil en clave experiencial implica superar nociones de ocio que asumen éste como sinónimo de actividad o tiempo libre, para otorgar relevancia a la calidad de las experiencias que este fenómeno desencadena. Este enfoque exige reconocer que no todas las experiencias y, por ende, todas las manifestaciones del ocio juvenil contribuyen de manera idéntica al desarrollo personal y social (Cuenca, 2015; Monteagudo, 2008).

El concepto de ocio valioso (Cuenca, 2015; Kleiber, Bayón, Cuenca y Monteagudo, 2014), acuñado por el Instituto de Estudios de Ocio de la Universidad de Deusto, permite poner el foco de atención en el estudio de aquellas experiencias (o estilos) de ocio que, siendo altamente significativas, mejoran en algún sentido a su protagonista. Con este concepto se quiere resaltar el potencial del ocio para dinamizar procesos individuales y/o colectivos de desarrollo humano a través de los múltiples beneficios que propicia (Cuenca, 2015; Kleiber, Walker y Mannell, 2011; Nussbaum, 2012; Porter, Iwasaki y Shank, 2010; Valdemoros, Ponce de León y Gradaille, 2016). Si esto es así, parece legítimo el interés científico y social que el ocio juvenil despierta, pues garantizar el cultivo de un ocio positivo, enriquecedor y saludable desde la infancia y juventud, es apostar por el desarrollo juvenil y su continuidad en etapas posteriores de la vida. 


\section{BENEFICIOS DEL OCIO JUVENIL}

El estudio de los beneficios del ocio ha sido abordado como antecedente, explorando las expectativas sobre los retornos positivos que se esperan obtener del ocio (Barnett, 2013; Gibson y Chang, 2012), o como consecuencia, a partir de sus efectos (Barnett, 2013; Codina, Pestana, Castillo y Balaguer, 2016; Sibthorp, Bialeschki, Morgan y Browne, 2013). Durante los últimos años, la literatura especializada se ha decantado por el uso del término resultados (outcomes) del ocio para destacar los efectos tanto positivos como negativos que el ocio puede promover (Kleiber, Walker y Mannel, 2011). Ante esta diversidad de posibilidades, conviene remarcar que este artículo centra la atención únicamente en los efectos positivos del ocio, es decir, en sus beneficios, considerando estos no desde las expectativas, sino en cuanto resultado obtenido del ocio juvenil.

Habitualmente, se entiende por beneficio "un cambio que se percibe como ventajoso, una mejora en algún aspecto que puede favorecer a la persona, grupo, sociedad o cualquier otra entidad" (Driver, Brown y Peterson, 1991, p. 4). Más tarde, Driver y Bruns (1999) avanzaron una triple acepción de este concepto que contempla todas sus vertientes; en primer lugar, como mejora de un estado o condición de una persona o colectivo; en segundo lugar, como prevención de una condición no deseada o el mantenimiento de una condición ya existente y deseada evitando la aparición de una condición no deseable; y, finalmente, como experiencia psicológica satisfactoria. La importancia de esta nueva definición radica en el reconocimiento del valor instrumental y final del ocio (Monteagudo, 2004). En ella se subraya la capacidad del ocio para ser un fin en sí mismo (valor final), fuente primera de satisfacción que no necesita de otros argumentos para justificar la acción, al tiempo que puede actuar como instrumento para el logro de otros objetivos (educativos, sociales, económicos, de salud...) (valor instrumental).

Aunque aún persiste en la literatura una tendencia a justificar la importancia del ocio juvenil, atendiendo únicamente a su naturaleza exotélica o instrumental, cada vez son más los enfoques que subrayan la naturaleza dual del ocio (final e instrumental). Larson (2000) insiste, por ejemplo, en la importancia de que las prácticas de ocio juveniles entusiasmen a este colectivo (valor final del ocio) al tiempo que promueven el desarrollo de habilidades necesarias para sus vidas (valor instrumental). Aún más, algunas investigaciones destacan el papel central de la satisfacción como condición "sine qua non" para la consecución de otros beneficios a través del ocio (Driver y Bruns, 1999; Monteagudo, Ahedo, Richter y Doistua, 2014).

Sibthorp, Bialeschki, Morgan y Browne (2013) estudian los beneficios que los campamentos de verano tienen en la población joven mediante una taxonomía de 11 tipos de beneficios: habilidades sociales, conductas promotoras 
del funcionamiento familiar, responsabilidad, independencia, habilidades de trabajo en grupo, competencia percibida, deseo de exploración, gusto por la Naturaleza, resolución de problemas, conexión con la vida del campamento y bienestar espiritual. Por su parte, Barnett (2013), en su estudio sobre el impacto de la personalidad en las expectativas que las y los jóvenes tienen sobre los retornos de su ocio, emplea una tipología de beneficios de 5 categorías: deseo de nuevas experiencias, deseo de retos y desarrollo de nuevas habilidades, deseo de refuerzos intrínsecos (satisfacción), deseo de relaciones sociales y, finalmente, deseo de ser una persona activa. Larson, Jarrett, Hansen, Pearse, Sullivan, Walker, Watkins y Wood (2004) destacan como consecuencias positivas de la participación de chicos y chicas adolescentes en programas de actividades extraescolares el desarrollo de la iniciativa personal, el fomento de la motivación intrínseca, la adquisición de habilidades sociales, el respeto a la diversidad y el cultivo de la responsabilidad.

\section{APORTACIONES DEL OCIO Y SUS BENEFICIOS AL DESARROLLO JUVENIL: FACTORES DETERMINANTES}

La revisión de estos y otros estudios que forman parte de la abundante literatura existente sobre los beneficios del ocio joven (Chawla, 2015; Doistua y Ried, 2016; Fraguela, Varela y Sanz, 2016; Gardner, Roth y Brooks-Gunn, 2008; Lazcano, Ortega y Baptista, 2015; Mahoney, Larson y Eccles, 2005; Oliva, Pertegal, Antolín, Reina y Ríos, 2011) permiten destacar dos cuestiones fundamentales. En primer lugar, se confirma que la mayoría de las investigaciones realizadas sobre el tema tienen como telón de fondo la teoría del desarrollo positivo juvenil (Damon, 2004) y el modelo de competencia, frente al modelo de déficit que inspiró buena parte de las iniciativas de ocio juvenil institucionales durante las últimas décadas del siglo XX. Desde este enfoque, la juventud ya no es un problema sino un recurso a desarrollar. De ahí que los programas de ocio que deseen contribuir al desarrollo juvenil deben promover una serie de beneficios que permitan a las y los jóvenes transitar con éxito por esta etapa vital y alcanzar una madurez ajustada (Parra, Oliva, Antolín, 2009). En segundo lugar, se evidencia que tanto los beneficios del ocio como el logro del desarrollo juvenil están mediados por variables diversas que han de considerarse al diseñar programas orientados a la promoción del ocio juvenil. Entre estas variables merece la pena destacar el carácter organizado o no organizado del ocio juvenil, los espacios en los que tiene lugar y el género.

- Ocio organizado versus no organizado

Desde el modelo de competencia que inspira la comprensión de la etapa juvenil como un periodo para el cultivo de capacidades (Damon, 2004; Parra, Oliva y Antolín, 2009), se defiende la importancia de que las y los jóvenes par- 
ticipen en programas de ocio organizado. Estos programas se llevan a cabo desde una institución y bajo la supervisión de una persona adulta y formada (Zeijl, Du Bois-Reymond y Te Poel, 2001) que orienta su intervención hacia la construcción de habilidades asociadas al desarrollo juvenil (Eccles y Gootman, 2002; Roth y Brooks-Gunn, 2003). Dichos programas disponen de unos objetivos claros que se pretenden alcanzar a través de actividades planificadas. Investigaciones previas demuestran que las y los jóvenes que participan en actividades de ocio organizadas perciben mayores beneficios que quienes practican su ocio de forma libre (Ahedo y Macua, 2016). Este tipo de ocio juvenil está asociado a un mayor compromiso social (Gardner, Roth y Brooks-Gunn, 2008), un mejor ajuste emocional (Broh, 2002) y una mayor disponibilidad de habilidades sociales (Devine y Parr, 2008). Estudios longitudinales confirman, además, los efectos positivos de estos programas, incluso una vez concluida la participación (Gardner, Roth y Brooks-Gunn, 2008). Los datos disponibles sobre el ocio juvenil revelan, sin embargo, que la mayoría de las prácticas de ocio joven son actividades no estructuradas (Mahoney, Larson y Eccles, 2005; Ramos, Rivera y Moreno, 2012).

- Espacios para el ocio juvenil

Los espacios para el ocio juvenil son componentes esenciales del mismo por su capacidad de modular las experiencias que acogen, así como los beneficios asociados a dichas experiencias. La literatura identifica una serie de rasgos que deberían compartir estos espacios cuando el objetivo a alcanzar es el desarrollo juvenil; entre otros, el acceso y uso de espacios física y psicológicamente seguros en los que sea posible la apropiación y construcción colectiva de significados. El carácter público o privado de estos espacios o la mayor o menor normativización adulta que prevalece en ellos (Tejerina, Carbajo y Martínez 2012) son también variables relevantes a la hora de entender el ocio juvenil. Las personas jóvenes buscan espacios que les proporcionen cierta distancia del mundo adulto (Abbot-Champan y Robertson, 2001) y obtienen más satisfacción en espacios en los que disfrutan de mayor autonomía y posibilidades de autogestión (Ortega, Lazcano y Baptista, 2015).

- Género

El género es una de las variables sociodemográficas que más incide en la configuración de las experiencias de ocio juvenil y en sus beneficios. Como variable independiente, el género parece modular las decisiones que la población toma respecto a su ocio: las modalidades que práctica, el tiempo dedicado, los motivos que respaldan la práctica o el tipo de barreras que percibe para continuar practicando (Henderson, 2009). Diferencias que se constatan ya durante 
la infancia y juventud y que, en muchos casos, persisten en etapas tardías de la vida (Kleiber, Bayón, Cuenca y Monteagudo, 2014). Investigaciones recientes (Barnett, 2013) revelan que las chicas son menos proclives que los chicos a buscar la adquisición de destrezas o habilidades, atribuyen menos importancia al disfrute y están menos predispuestas a permanecer activas a través de su ocio.

\section{MÉTODO}

\section{Participantes}

Este trabajo se enmarca dentro del proyecto coordinado "De los tiempos educativos a los tiempos sociales: la construcción cotidiana de la condición juvenil en una sociedad de redes", cuyo universo es la población joven española (16-18 años). Se ha utilizado un diseño metodológico cuantitativo y un muestreo aleatorio simple entre las y los jóvenes que cursaban Educación Secundaria Postobligatoria (Bachiller, Formación Profesional de Grado Medio y Programas de Capacitación Profesional Inicial) en centros públicos y privados del territorio español, tratando de mantener una afijación proporcional en cada una de las seis áreas en las que se dividió el territorio español: Noreste: Cataluña, Baleares y Aragón (excepto Teruel incluida en el área Centro); Levante: Comunidad Valenciana, Murcia y Albacete; Sur: Andalucía, Islas Canarias, Ceuta y Melilla; Centro: Madrid, Castilla-La Mancha, Castilla-León, Cáceres y Teruel (excepto Albacete, León, Palencia y Burgos incluidas en las áreas Norte y Noroeste); Noroeste: Galicia, Asturias y León; Norte: Cantabria, País Vasco, La Rioja, Navarra, Burgos y Palencia.

La muestra está compuesta por un total de 1253 jóvenes (48\% chicas y $52 \%$ chicos). Se trata de una muestra representativa con un error del 2,3\% y un nivel de confianza del 95\%.

\section{Instrumento}

Se elaboró un cuestionario ad hoc, orientado a dar respuesta a los objetivos planteados para el conjunto de la investigación coordinada. El cuestionario abarcaba un amplio espectro de cuestiones centradas en 7 grandes bloques: ocio y tiempo libre, vida familiar y en el centro escolar, salud y calidad de vida así como mercado laboral y emprendimiento, además de las variables sociodemográficas. Este artículo se centra únicamente en el apartado dedicado al ocio y tiempo libre y, fundamentalmente, en las siguientes variables:

Actividades de ocio más significativas (variable independiente): cada joven debía seleccionar, entre todas las actividades de ocio practicadas, las tres más 
significativas; es decir, aquellas que consideraba especialmente importantes y de las que no le gustaría prescindir. Aunque era una pregunta abierta, disponía de una ficha de apoyo en la que se presentaban distintas actividades de ocio agrupadas en 8 categorías: actividades culturales (escuchar música, ver televisión o películas, ir al cine, teatro, museos o exposiciones, asistir a conciertos o espectáculos deportivos, realizar alguna actividad artística...), ocio digital (escribir un blog o crear páginas web, participar en chats o redes sociales, videojuegos, juegos de azar online..), actividad físico-deportiva (individuales, grupales, de cooperación u oposición...), turismo o excursionismo, fiestas y celebraciones, juegos sin ordenador, hobbies y asociacionismo/voluntariado.

Beneficios del ocio juvenil más significativo (variable dependiente). Mediante una escala tipo Likert de 5 puntos que iba desde 1 . Nada de acuerdo hasta 5 . Muy de acuerdo, se les solicitaba su grado de acuerdo con un total de 5 afirmaciones, cada una asociada a la percepción de un tipo de beneficio: a) Estoy más en forma, controlo mejor mis movimientos, mantengo o mejoro mi condición física, etc. (Beneficio físico); b) Me siento más satisfecho/a, disfruto haciéndolo, me divierte, etc. (Beneficio emocional); c) Soy más creativo/a, adquiero conocimientos, aprendo cosas, amplio mi mundo, etc. (Beneficio cognitivo); d) Desarrollo nuevas destrezas manuales y adquiero o perfecciono habilidades técnicas (Adquisición de destrezas y habilidades técnicas); e) Hago cosas diferentes con más gente, me siento parte de un grupo, me ayuda a relacionarme mejor con los demás, etc. (Beneficio social).

Carácter organizado, espontáneo o ambos del ocio juvenil más significativo (variable independiente). Mediante una pregunta simple, de respuesta si/no, debían indicar si realizaban sus prácticas de ocio seleccionadas en el marco de una organización, por su cuenta o de ambas maneras.

Espacios para el ocio juvenil más significativo (variable independiente). Para el análisis de los espacios en los que tenían lugar las actividades de ocio seleccionadas se recodificaron las 8 opciones de respuesta proporcionadas en tres tipos de espacios: espacios abiertos (espacios naturales y espacios públicos abiertos como la calle, parques o plazas); espacios cerrados particulares (locales privados, cedidos a alquilados que se comparten con gente de la misma edad; la casa, urbanizaciones u otras residencias privadas; asociaciones, clubes, sociedades o peñas donde hay gente de distintas edades); espacios cerrados ajenos (espacios municipales como polideportivos, centros culturales, ludotecas; las instalaciones del centro escolar; espacios de ocio como centros comerciales, zonas de bares, cines y discotecas). 


\section{Procedimiento}

La recogida de información se realizó de forma presencial en centros escolares de las diferentes Comunidades Autónomas del contexto español. Una vez contactados los centros educativos, se solicitó a las y los directores su colaboración y se seleccionaron aleatoriamente las clases en las que se aplicaría el cuestionario. El trabajo de campo se realizó de octubre de 2014 a febrero de 2015. Dos investigadores se desplazaron a cada centro educativo donde se administró el cuestionario en un aula de forma grupal. La participación era voluntaria y anónima.

\section{Análisis de datos}

Para el análisis de los datos se utilizó el software SPSS (v. 21). El análisis descriptivo del ocio juvenil, su organización, así como los lugares en los que se desarrolla se realiza mediante frecuencias y porcentajes. El análisis de los beneficios se presenta mediante las correspondientes medias y desviaciones típicas. Por último, la asociación de los beneficios con las variables género, edad, estudios, organización de las actividades y lugar de realización se lleva a cabo mediante la prueba U de Mann-Whitney.

\section{Resultados}

Esta sección presenta los resultados obtenidos en relación con la percepción de beneficios derivados de las prácticas de ocio de las y los jóvenes españoles (16-18 años). En los siguientes apartados se abordará la relación entre estos beneficios y el género, la modalidad de ocio practicada, el carácter organizado o no organizado de la actividad y el lugar en el que se realiza. Los datos obtenidos no muestran diferencias significativas entre percepción de beneficios y la edad o el tipo de estudios. Por lo que no se incidirá nuevamente en el estudio de estas variables.

\section{BENEFICIOS DEL OCIO JUVENIL EN ESPAÑA}

La población joven española percibe principalmente tres tipos de beneficios asociados a sus prácticas de ocio más significativas: beneficios emocionales (media de 4,38 sobre 5 ), sociales $(3,48)$ y cognitivos $(3,46)$.

Esta relevancia se mantiene e incluso se hace más evidente, considerando de manera independiente el orden de importancia de las prácticas de ocio juvenil más significativas. Las puntuaciones medias de los beneficios emocionales son notablemente más altas que las del resto en las tres actividades (Tabla 1). 
Tabla 1. Puntuaciones medias de percepción de beneficios de las tres actividades de ocio juvenil más significativas en conjunto y, según importancia de cada actividad

\begin{tabular}{|c|c|c|c|c|c|c|c|c|}
\hline & \multicolumn{2}{|c|}{$\begin{array}{l}\text { Conjunto de } \\
\text { actividades }\end{array}$} & \multicolumn{2}{|c|}{$\begin{array}{l}1^{\mathrm{a}} \text { Actividad } \\
\text { de ocio }\end{array}$} & \multicolumn{2}{|c|}{$\begin{array}{l}2^{\mathrm{a}} \text { Actividad } \\
\text { de ocio }\end{array}$} & \multicolumn{2}{|c|}{$\begin{array}{c}3^{\text {a }} \text { Actividad } \\
\text { de ocio }\end{array}$} \\
\hline & Media & $D E$ & Media & $D E$ & Media & $D E$ & Media & $D E$ \\
\hline Beneficios físicos & 2,69 & 1,60 & 3,00 & 1,66 & 2,66 & 1,59 & 2,39 & 1,48 \\
\hline Beneficios emocionales & 4,38 & 0,92 & 4,54 & 0,82 & 4,39 & 0,90 & 4,22 & 1,00 \\
\hline Beneficios cognitivos & 3,46 & 1,28 & 3,56 & 1,30 & 3,44 & 1,27 & 3,36 & 1,27 \\
\hline $\begin{array}{l}\text { Adquisición destrezas y } \\
\text { habilidades técnicas }\end{array}$ & 2,85 & 1,51 & 3,13 & 1,56 & 2,80 & 1,48 & 2,62 & 1,45 \\
\hline Beneficios sociales & 3,48 & 1,48 & 3,84 & 1,41 & 3,42 & 1,47 & 3,16 & 1,49 \\
\hline Beneficio medio & 3,40 & 0,93 & 3,63 & 0,94 & 3,37 & 0,91 & 3,18 & 0,89 \\
\hline
\end{tabular}

Tabla 2. Puntuaciones medias de percepción de beneficios del ocio juvenil más significativo, según género

\begin{tabular}{|c|c|c|c|c|c|c|c|}
\hline & \multicolumn{3}{|c|}{ Género } & \multirow{2}{*}{\multicolumn{4}{|c|}{ Estadísticos de contraste }} \\
\hline & \multirow{2}{*}{\begin{tabular}{|l} 
Mujer \\
Media
\end{tabular}} & \multirow{2}{*}{$\begin{array}{c}\text { Hombre } \\
\text { Media }\end{array}$} & \multirow{2}{*}{\begin{tabular}{|c|} 
Total \\
Media
\end{tabular}} & & & & \\
\hline & & & & $\begin{array}{l}\text { U de Mann- } \\
\text { Whitney }\end{array}$ & $\begin{array}{c}W \text { de } \\
\text { Wilcoxon }\end{array}$ & $z$ & $\begin{array}{c}\text { Sig. asintót. } \\
\text { (bilateral) }\end{array}$ \\
\hline $\begin{array}{l}\text { Beneficios } \\
\text { físicos }\end{array}$ & 2,43 & 2,93 & 2,69 & 910801 & 1915454,000 & $-8,390$ & 0,000 \\
\hline $\begin{array}{l}\text { Beneficios } \\
\text { emocionales }\end{array}$ & 4,37 & 4,39 & 4,38 & 1147531,5 & 2228716,500 & $-1,158$ & 0,247 \\
\hline $\begin{array}{l}\text { Beneficios } \\
\text { cognitivos }\end{array}$ & 3,36 & 3,55 & 3,46 & 1053603 & 2117214,000 & $-3,829$ & 0,000 \\
\hline $\begin{array}{l}\text { Adquisición } \\
\text { destrezas y } \\
\text { habilidades } \\
\text { técnicas }\end{array}$ & 2,50 & 3,18 & 2,85 & 832334,5 & 1861229,500 & $-12,350$ & 0,000 \\
\hline $\begin{array}{l}\text { Beneficios } \\
\text { sociales }\end{array}$ & 3,33 & 3,62 & 3,48 & 1013086 & 2059267,000 & $-5,110$ & 0,000 \\
\hline $\begin{array}{l}\text { Beneficio } \\
\text { medio }\end{array}$ & 3,23 & 3,56 & 3,40 & 980849 & 2124677,000 & $-9,839$ & 0,000 \\
\hline
\end{tabular}


Este dato es importante porque subraya el interés intrínseco de las y los jóvenes en las actividades indicadas. Quienes perciben este tipo de beneficio afirman que su satisfacción deriva de hacer aquello que les gusta y satisface. En este orden, los beneficios sociales y cognitivos son también importantes.

\section{LOS BENEFICIOS DEL OCIO JUVENIL, SEGÚN GÉNERO}

El género emerge como una variable relevante a la hora de analizar los beneficios que la población joven obtiene de su ocio (Tabla 2). Las chicas presentan medias inferiores que las de los chicos en todos los tipos de beneficios, excepto en el caso de los emocionales.

Estas diferencias resultan estadísticamente significativas y se hacen especialmente notorias cuando se trata de la adquisición de destrezas y habilidades técnicas (diferencia de 0,68 puntos a favor de los chicos) y de beneficios físicos (diferencia de 0,5 puntos a favor de los chicos).

\section{RELACIÓN DEL TIPO DE ACTIVIDAD DE OCIO CON LOS BENEFICIOS PERCIBIDOS}

Consideradas en su conjunto, las tres actividades que el colectivo joven indica como sus prácticas de ocio más significativas son las culturales $(25,4 \%)$, las físico-deportivas $(21,45)$ y las fiestas y celebraciones $(14,2 \%)$ (Tabla 3). El $22 \%$ de las y los jóvenes opta por la categoría "Otras actividades", en la que destacan las respuestas que consideran "estar con las amistades" como actividad de ocio prioritaria.

El análisis individual de cada una de las tres prácticas de ocio identificadas como más significativas revela que la actividad principal es la físico-deportiva $(29,7 \%)$; le siguen las prácticas culturales $(20,9 \%)$ y las fiestas y celebraciones (17\%). A mayor distancia, destaca el ocio digital como actividad más relevante para el 9,5\% (Tabla 3). Las actividades culturales adquieren protagonismo como segundas prácticas de ocio prioritarias $(27,1 \%)$ y el ocio digital se equipara en importancia al ocio físico-deportivo cuando se trata de las terceras prácticas de ocio más significativas ( $12,3 \%$ y $13,6 \%$, respectivamente) (Tabla 3).

El cruce del tipo de práctica con la naturaleza de los beneficios percibidos permite detectar los beneficios que las y los jóvenes identifican como consecuencia de sus prácticas de ocio preferidas (Tabla 4). Los beneficios físicos están asociados preferentemente a las actividades físico-deportivas, mientras que apenas se relacionan con el ocio digital. Los beneficios emocionales son los más habituales en todas las actividades pero su percepción es especialmente eleva- 
Tabla 3. Práctica de las actividades de ocio juvenil más significativas en conjunto y según importancia de cada actividad ( $\mathrm{n}$ actividades=3759)

\begin{tabular}{|c|c|c|c|c|c|c|c|c|}
\hline & \multicolumn{2}{|c|}{$\begin{array}{l}\text { Conjunto de } \\
\text { actividades }\end{array}$} & \multicolumn{2}{|c|}{$\begin{array}{l}1^{\mathrm{a}} \text { Actividad } \\
\text { de ocio }\end{array}$} & \multicolumn{2}{|c|}{$\begin{array}{l}2^{\text {a }} \text { Actividad } \\
\text { de ocio }\end{array}$} & \multicolumn{2}{|c|}{$\begin{array}{c}3^{\mathrm{a}} \text { Actividad } \\
\text { de ocio }\end{array}$} \\
\hline & $\mathrm{N}$ & $\%$ & $\mathrm{~N}$ & $\%$ & $\mathbf{N}$ & $\%$ & $\mathrm{~N}$ & $\%$ \\
\hline Actividades culturales & 955 & $25,4 \%$ & 262 & $20,9 \%$ & 340 & $27,1 \%$ & 353 & $28,2 \%$ \\
\hline Ocio Digital & 407 & $10,8 \%$ & 119 & $9,5 \%$ & 134 & $10,7 \%$ & 154 & $12,3 \%$ \\
\hline $\begin{array}{l}\text { Actividad físico- } \\
\text { deportiva }\end{array}$ & 804 & $21,4 \%$ & 372 & $29,7 \%$ & 261 & $20,8 \%$ & 171 & $13,6 \%$ \\
\hline $\begin{array}{l}\text { Turismo o } \\
\text { Excursionismo }\end{array}$ & 75 & $2,0 \%$ & 13 & $1,0 \%$ & 21 & $1,7 \%$ & 41 & $3,3 \%$ \\
\hline Fiestas y Celebraciones & 534 & $14,2 \%$ & 213 & $17,0 \%$ & 163 & $13,0 \%$ & 158 & $12,6 \%$ \\
\hline Juegos & 19 & $0,5 \%$ & 5 & $0,4 \%$ & 4 & $0,3 \%$ & 10 & $0,8 \%$ \\
\hline Hobbies & 95 & $2,5 \%$ & 9 & $0,7 \%$ & 37 & $3,0 \%$ & 49 & $3,9 \%$ \\
\hline $\begin{array}{l}\text { Asociacionismo- } \\
\text { Voluntariado }\end{array}$ & 18 & $0,5 \%$ & 6 & $0,5 \%$ & 5 & $0,4 \%$ & 7 & $0,6 \%$ \\
\hline Otros - Varios & 852 & $22,7 \%$ & 254 & $20,3 \%$ & 288 & $23,0 \%$ & 310 & $24,7 \%$ \\
\hline
\end{tabular}

da en las actividades físico-deportivas (media de 4,59 sobre 5), en fiestas y celebraciones y en el ámbito del asociacionismo y voluntariado (medias de 4,57, en ambos casos) así como en el turismo y excursionismo (media de 4,53). Por contraste, en esta misma categoría de beneficios, el ocio digital es la actividad que muestra una media más baja (media de 4,08). Por su parte, los beneficios cognitivos derivan de prácticas como el asociacionismo y voluntariado $(4,54)$, el turismo o excursionismo $(4,05)$, así como de las actividades culturales $(3,75)$. La adquisición de destrezas y habilidades técnicas se asocia principalmente con la actividad físico-deportiva $(3,98)$, mientras que apenas mantiene relación con el ámbito de las fiestas y celebraciones $(2,05)$. Finalmente, la contribución del ocio al mundo social de las y los jóvenes adopta su máxima expresión en el mundo del asociacionismo y voluntariado, en las fiestas y celebraciones y en la realización de actividades físico-deportivas (4,79, 4,47 y 4,02, respectivamente).

\section{LA ORGANIZACIÓN DEL OCIO JUVENIL Y SU RELACIÓN CON LA PERCEPCIÓN DE BENEFICIOS}

El carácter organizado o espontáneo del ocio joven es otra variable a tener en cuenta en el análisis de la percepción de beneficios. El 60,9\% de las actividades 
Tabla 4. Puntuaciones medias de percepción de beneficios de las tres actividades de ocio juvenil más significativas en conjunto, según tipo de actividad

\begin{tabular}{|c|c|c|c|c|c|c|}
\hline & $\begin{array}{c}\text { Beneficios } \\
\text { físicos }\end{array}$ & $\begin{array}{c}\text { Beneficios } \\
\text { emocionales }\end{array}$ & \begin{tabular}{|l|} 
Beneficios \\
cognitivos
\end{tabular} & $\begin{array}{c}\text { Adquisición } \\
\text { destrezas y } \\
\text { habilidades } \\
\text { técnicas }\end{array}$ & $\begin{array}{l}\text { Beneficios } \\
\text { sociales }\end{array}$ & $\begin{array}{l}\text { Beneficio } \\
\text { medio }\end{array}$ \\
\hline $\begin{array}{l}\text { Actividades } \\
\text { culturales }\end{array}$ & 1,80 & 4,25 & 3,75 & 2,41 & 2,45 & 2,98 \\
\hline Ocio Digital & 1,72 & 4,08 & 3,51 & 2,80 & 3,14 & 3,09 \\
\hline $\begin{array}{l}\text { Actividad físico- } \\
\text { deportiva }\end{array}$ & 4,50 & 4,59 & 3,52 & 3,98 & 4,02 & 4,13 \\
\hline $\begin{array}{l}\text { Turismo o } \\
\text { Excursionismo }\end{array}$ & 3,44 & 4,53 & 4,05 & 2,82 & 3,87 & 3,78 \\
\hline $\begin{array}{l}\text { Fiestas y } \\
\text { Celebraciones }\end{array}$ & 2,13 & 4,57 & 2,89 & 2,05 & 4,47 & 3,27 \\
\hline Juegos & 2,13 & 4,13 & 2,94 & 3,13 & 3,75 & 3,26 \\
\hline Hobbies & 2,46 & 4,32 & 2,83 & 2,36 & 3,10 & 3,04 \\
\hline $\begin{array}{l}\text { Asociacionismo- } \\
\text { Voluntariado }\end{array}$ & 2,77 & 4,57 & 4,54 & 3,08 & 4,79 & 4,01 \\
\hline Otros - Varios & 2,72 & 4,36 & 3,39 & 2,87 & 3,65 & 3,43 \\
\hline Total & 2,69 & 4,38 & 3,46 & 2,85 & 3,48 & 3,40 \\
\hline \multicolumn{7}{|c|}{ Estadísticos de contraste } \\
\hline Chi-cuadrado & 1209,285 & 109,732 & 175,212 & 554,594 & 688,606 & 673,526 \\
\hline gl & 7,000 & 7,000 & 7,000 & 7,000 & 7,000 & 7,000 \\
\hline Sig. Asintót. & 0,000 & 0,000 & 0,000 & 0,000 & 0,000 & 0,000 \\
\hline
\end{tabular}

del ocio juvenil se realiza de forma libre, al margen de cualquier entidad (Tabla 5). Este porcentaje es especialmente elevado en el caso del ocio digital (84\%) o las actividades culturales $(75,7 \%)$, pero se reduce considerablemente en actividades como asociacionismo/voluntariado $(11,1 \%)$ y el ocio físico-deportivo $(34,5 \%)$. Se trata en ambos casos de actividades que se llevan a cabo, sobre todo, en el marco de una organización (55,6\% y 36,6\%, respectivamente).

Los datos confirman el impacto positivo del ocio organizado en la percepción de beneficios. La puntuación media de beneficios alcanza un 3,85 sobre 5 cuando se trata de un ocio organizado, mientras que desciende hasta el 3,19 en 
Tabla 5. Práctica de las actividades de ocio juvenil más significativas en conjunto y según su carácter organizado o espontáneo (n actividades $=3645$ )

\begin{tabular}{|c|c|c|c|c|c|c|}
\hline & \multicolumn{6}{|c|}{ Organización } \\
\hline & \multicolumn{2}{|c|}{$\begin{array}{l}\text { De forma libre, } \\
\text { por mi cuenta }\end{array}$} & \multicolumn{2}{|c|}{$\begin{array}{l}\text { De forma } \\
\text { organizada, en } \\
\text { una asociación, } \\
\text { club, etc. }\end{array}$} & \multicolumn{2}{|c|}{ Ambas } \\
\hline & $\mathrm{N}$ & $\%$ & $\mathrm{~N}$ & $\%$ & $\mathrm{~N}$ & $\%$ \\
\hline Actividades culturales & 723 & $75,7 \%$ & 53 & $5,5 \%$ & 109 & $11,4 \%$ \\
\hline Ocio Digital & 342 & $84,0 \%$ & 11 & $2,7 \%$ & 25 & $6,1 \%$ \\
\hline Actividad físico-deportiva & 277 & $34,5 \%$ & 294 & $36,6 \%$ & 176 & $21,9 \%$ \\
\hline Turismo o Excursionismo & 36 & $48,0 \%$ & 16 & $21,3 \%$ & 16 & $21,3 \%$ \\
\hline Fiestas y Celebraciones & 322 & $60,3 \%$ & 100 & $18,7 \%$ & 75 & $14,0 \%$ \\
\hline Juegos & 11 & $57,9 \%$ & 2 & $10,5 \%$ & 5 & $26,3 \%$ \\
\hline Hobbies & 68 & $71,6 \%$ & 6 & $6,3 \%$ & 10 & $10,5 \%$ \\
\hline $\begin{array}{l}\text { Asociacionismo- } \\
\text { Voluntariado }\end{array}$ & 2 & $11,1 \%$ & 10 & $55,6 \%$ & 6 & $33,3 \%$ \\
\hline Otros - Varios & 440 & $59,6 \%$ & 124 & $16,8 \%$ & 92 & $12,5 \%$ \\
\hline Total & 2221 & $60,9 \%$ & 616 & $16,9 \%$ & 514 & $14,1 \%$ \\
\hline
\end{tabular}

el caso del ocio que las y los jóvenes realizan por su cuenta (Tabla 6). Esta diferencia a favor del ocio organizado se confirma en todos los tipos de beneficios, excepto en los emocionales. El único tipo de beneficio que se obtiene a través del ocio organizado y no organizado.

\section{LOS ESPACIOS DE OCIO JUVENIL Y SU CONEXIÓN CON LOS BENEFICIOS PERCIBIDOS}

Con el fin de conocer la naturaleza de los espacios en los que transcurre el ocio juvenil en España, se presentan los resultados obtenidos en las tres categorías de espacios consideradas: abiertos, cerrados particulares y cerrados ajenos (Tabla 7).

Los espacios públicos abiertos acogen principalmente el ocio físico-deportivo $(23,8 \%)$ que se realiza en la calle, en lugares públicos, en muchos casos, habilitados para ello como pueden ser plazas o parques. Estos espacios son también principales aliados de fiestas y celebraciones (23,7\%). Los espacios cerrados 
Tabla 6. Puntuaciones medias de los beneficios percibidos de las tres actividades de ocio juvenil más significativas en conjunto, según su carácter organizado o espontáneo (n actividades=3759)

\begin{tabular}{|c|c|c|c|c|c|c|}
\hline & $\begin{array}{c}\text { Beneficios } \\
\text { físicos } \\
\text { Media }\end{array}$ & $\begin{array}{c}\text { Beneficios } \\
\text { emocionales } \\
\text { Media }\end{array}$ & $\begin{array}{c}\text { Beneficios } \\
\text { cognitivos } \\
\text { Media }\end{array}$ & \begin{tabular}{|c|} 
Adquisición \\
destrezas y \\
habilidades \\
técnicas \\
Media \\
\end{tabular} & $\begin{array}{c}\text { Beneficios } \\
\text { sociales } \\
\text { Media }\end{array}$ & $\begin{array}{c}\text { Total } \\
\text { Media }\end{array}$ \\
\hline $\begin{array}{l}\text { De forma libre, } \\
\text { por mi cuenta } \\
\text { (no organizado) }\end{array}$ & 2,29 & 4,34 & 3,41 & 2,55 & 3,15 & 3,19 \\
\hline $\begin{array}{l}\text { De forma } \\
\text { organizada, en } \\
\text { una asociación, } \\
\text { club, etc. }\end{array}$ & 3,50 & 4,52 & 3,50 & 3,46 & 4,24 & 3,85 \\
\hline Ambas & 3,38 & 4,50 & 3,61 & 3,35 & 3,99 & 3,77 \\
\hline $\mathrm{Ns} / \mathrm{Nc}$ & 2,76 & 4,14 & 3,44 & 2,99 & 3,42 & 3,43 \\
\hline Total & 2,69 & 4,38 & 3,46 & 2,85 & 3,48 & 3,40 \\
\hline \multicolumn{7}{|c|}{ Estadísticos de contraste } \\
\hline Chi-cuadrado & 0,000 & 0,000 & 0,009 & 0,000 & 0,000 & 0,000 \\
\hline gl & 2,000 & 2,000 & 2,000 & 2,000 & 2,000 & 2,000 \\
\hline Sig. asintót. & 319,607 & 22,256 & 9,444 & 201,495 & 281,825 & 309,789 \\
\hline
\end{tabular}

propios emergen como contextos idóneos para actividades culturales $(31,1 \%)$ y también para fiestas y celebraciones $(17,4 \%)$. Por su parte los espacios cerrados ajenos son propicios para el ocio físico-deportivo $(47,5 \%)$ y para algunas actividades culturales $(17,9 \%)$. El porcentaje de actividades culturales que tiene lugar en espacios cerrados ajenos es inferior al de las que transcurren en espacios cerrados propios tales como la propia casa o locales particulares, cedidos o alquilados que, en algunas comunidades autónomas reciben el nombre de lonjas. Dichas lonjas son casi siempre espacios auto-gestionados por las y los propios jóvenes y en los que disfrutan de prácticas culturales, así como de fiestas y celebraciones particulares. Finalmente, los espacios cerrados ajenos (polideportivos o instalaciones del centro escolar, entre otros) acogen casi la mitad de las actividades físico-deportivas $(47,5 \%)$ y, en menor medida, algunas actividades culturales $(17,9 \%)$. Dado que se trata de espacios cerrados ajenos bien puede pensarse en cines, centros culturales o ludotecas, como algunos de los espacios que utiliza el colectivo joven para estas actividades culturales. 
Tabla 7. Espacios en los que tiene lugar ocio juvenil más significativo ( $\mathrm{n}$ actividades $=3759$ )

\begin{tabular}{l|c|c|c|c|c|c}
\hline & \multicolumn{2}{|c|}{$\begin{array}{c}\text { Espacios públicos } \\
\text { abiertos }\end{array}$} & $\begin{array}{c}\text { Espacios cerrados } \\
\text { propios }\end{array}$ & \multicolumn{2}{|c}{$\begin{array}{c}\text { Espacios } \\
\text { cerrados ajenos }\end{array}$} \\
\hline & $\mathrm{N}$ & $\%$ & $\mathrm{~N}$ & $\%$ & $\mathrm{~N}$ & $\%$ \\
\hline Actividades culturales & 158 & $13,7 \%$ & 765 & $31,1 \%$ & 142 & $17,9 \%$ \\
\hline Ocio Digital & 71 & $6,2 \%$ & 352 & $14,3 \%$ & 57 & $7,2 \%$ \\
\hline Actividad físico-deportiva & 274 & $23,8 \%$ & 335 & $13,6 \%$ & 377 & $47,5 \%$ \\
\hline Turismo o Excursionismo & 52 & $4,5 \%$ & 13 & $0,5 \%$ & 5 & $0,6 \%$ \\
\hline Fiestas y Celebraciones & 272 & $23,7 \%$ & 427 & $17,4 \%$ & 42 & $5,3 \%$ \\
\hline Juegos & 2 & $0,2 \%$ & 14 & $0,6 \%$ & 4 & $0,5 \%$ \\
\hline Hobbies & 20 & $1,7 \%$ & 74 & $3,0 \%$ & 3 & $0,4 \%$ \\
\hline $\begin{array}{l}\text { Asociacionismo- } \\
\text { Voluntariado }\end{array}$ & 6 & $0,5 \%$ & 11 & $0,4 \%$ & 8 & $1,05 \%$ \\
\hline Otros - Varios & 295 & $25,7 \%$ & 468 & $19,0 \%$ & 156 & $19,6 \%$ \\
\hline
\end{tabular}

La consideración de los beneficios, atendiendo al tipo de espacios indica que los beneficios físicos están asociados a espacios cerrados ajenos $(3,61)$, en los que transcurre buena parte de su ocio físico-deportivo (Tabla 8). A pesar de que los beneficios emocionales son elevados en todos los tipos de espacios, los espacios públicos abiertos son el principal marco para la percepción de estos retornos $(4,54)$. Los beneficios cognitivos son ligeramente más habituales en espacios cerrados ajenos $(3,63)$. La adquisición de destrezas y habilidades técnicas tiene lugar principalmente en espacios cerrados ajenos $(3,55)$. Finalmente, el cultivo del mundo social de las y los jóvenes se lleva a cabo sobre todo en espacios cerrados ajenos $(4,02)$ y en públicos abiertos $(3,88)$. Recordemos que espacios de ocio como centros comerciales, zonas de bares, cines y discotecas están considerados en esta categoría, espacios cerrados ajenos.

\section{DISCUSIÓN Y CONCLUSIONES}

Este estudio sobre el ocio de la población joven española (16-18 años) y los beneficios que este ámbito les reporta centra su atención en aquellas prácticas de ocio que las y los propios jóvenes identifican como significativas, esto es, aquellas que les resultan especialmente importantes y de las cuales no quisieran prescindir. Este enfoque, totalmente en línea con la comprensión del ocio joven en 
Tabla 8. Puntuaciones medias de los beneficios percibidos de las tres actividades de ocio juvenil más significativas, según el tipo de espacio ( $\mathrm{n}$ actividades $=3759$ )

\begin{tabular}{l|c|c|c|c|c|c}
\hline & $\begin{array}{c}\text { Beneficios } \\
\text { físicos } \\
\text { Media }\end{array}$ & $\begin{array}{c}\text { Beneficios } \\
\text { emocionales } \\
\text { Media }\end{array}$ & $\begin{array}{c}\text { Beneficios } \\
\text { cognitivos } \\
\text { Media }\end{array}$ & $\begin{array}{c}\text { Adquisición } \\
\text { destrezas y } \\
\text { habilidades } \\
\text { técnicas } \\
\text { Media }\end{array}$ & $\begin{array}{c}\text { Beneficios } \\
\text { sociales } \\
\text { Media }\end{array}$ & $\begin{array}{c}\text { Beneficio } \\
\text { medio } \\
\text { Media }\end{array}$ \\
\hline $\begin{array}{l}\text { Espacios } \\
\text { públicos } \\
\text { abiertos }\end{array}$ & 2,97 & 4,54 & 3,45 & 2,84 & 3,88 & 3,55 \\
\hline $\begin{array}{l}\text { Espacios } \\
\text { cerrados } \\
\text { particulares }\end{array}$ & 2,36 & 4,36 & 3,43 & 2,66 & 3,39 & 3,27 \\
\hline $\begin{array}{l}\text { Espacios } \\
\text { cerrados ajenos }\end{array}$ & 3,61 & 4,47 & 3,63 & 3,55 & 4,02 & 3,87 \\
\hline
\end{tabular}

Estadísticos de contraste (Sig. Asintót bilateral)

\begin{tabular}{l|c|c|c}
\hline & $\begin{array}{c}\text { Espacios públicos } \\
\text { abiertos }\end{array}$ & $\begin{array}{c}\text { Espacios cerrados } \\
\text { particulares }\end{array}$ & $\begin{array}{c}\text { Espacios cerrados } \\
\text { ajenos }\end{array}$ \\
\hline Beneficios físicos & 0,000 & 0,000 & 0,000 \\
\hline Beneficios emocionales & 0,000 & 0,012 & 0,002 \\
\hline Beneficios cognitivos & 0,662 & 0,094 & 0,000 \\
\hline $\begin{array}{l}\text { Adquisición destrezas } \\
\text { y habilidades técnicas }\end{array}$ & 0,878 & 0,000 & 0,000 \\
\hline Beneficios sociales & 0,000 & 0,000 & 0,000 \\
\hline Beneficio medio & 0,000 & 0,000 & 0,000 \\
\hline
\end{tabular}

clave de experiencia valiosa, proporciona un punto de partida novedoso desde el que analizar los beneficios del ocio.

Tres son los tipos de beneficios principales que la población joven española obtiene de su ocio significativo: emocionales, sociales y cognitivos. Su ocio más significativo es, para este colectivo, fuente inagotable de beneficios emocionales. Estos beneficios, asociados a la satisfacción de realizar aquello que realmente les gusta, revelan la orientación intrínseca de las y los jóvenes españoles hacia su ocio, cuya práctica constituye en sí misma un beneficio. Los beneficios emocionales son los que presentan puntuaciones más altas y su protagonismo no varía en función de las variables analizadas. El ocio juvenil más sig- 
nificativo proporciona además una alta dosis de beneficios sociales y cognitivos. Ambos tipos de beneficios han sido destacados en la literatura por ser elementos indispensables para el ajuste psicológico y la formación de la identidad que conlleva el desarrollo juvenil (Zarret, Fay, Carrano, Phelps y Letner, 2009).

Las tres prácticas de ocio joven más significativas son, según su importancia, el ocio físico-deportivo, las actividades culturales y las fiestas y celebraciones. Las actividades físico-deportivas son las que reportan una mayor percepción de beneficios, principalmente de tipo físico, emocional, así como la adquisición de destrezas y habilidades técnicas. Resultados similares se obtuvieron en otros estudios como el realizado por Ponce de León, Sanz y Valdemoros (2015). Así, el ocio físico-deportivo no es solo la práctica más significativa, sino también la que les proporciona un abanico más amplio de retornos positivos. Las fiestas y celebraciones, tercer tipo de actividad preferida, les proporcionan beneficios emocionales y sociales, esenciales en esta etapa vital. Sin embargo, llama la atención que las actividades culturales, elegidas por el colectivo joven como uno de sus ocios prioritarios, sea una fuente de beneficios más comedida que otras actividades menos significativas. El ocio cultural reporta, sobre todo, beneficios emocionales y cognitivos, pero en menor medida que prácticas como el turismo/excursionismo o el voluntariado/asociacionismo, actividades que resultan prioritarias para un porcentaje menor de jóvenes y que, sin embargo, para el escaso porcentaje que las practica son fuente de importantes beneficios emocionales, cognitivos y especialmente, sociales. En cuanto al ocio digital, cabe reseñar que, a pesar de ser una práctica muy extendida entre la población joven (Colás, González y de Pablos, 2013), este estudio evidencia que no ocupa un lugar privilegiado entre las prácticas de ocio más significativas de este colectivo (Viñals y Cuenca, 2016) y que la puntuación media de los beneficios que reporta tampoco se halla entre las más altas $(3,09)$. Tales resultados evidencian una aparente contradicción en la que se hace necesario profundizar para esclarecer los motivos de la elevada penetración social de este tipo de ocio. ¿Por qué una actividad de ocio que no es especialmente significativa para las y los jóvenes constituye una práctica cotidiana, plenamente instaurada en su rutina diaria? Aún más, ¿qué factores explican la expansión y persistencia de este hábito si los beneficios que reporta no son los más destacables?

El género emerge como una variable moduladora de los beneficios de ocio juvenil. Los resultados confirman, en primer lugar, que las mujeres muestran, en todos los tipos de beneficio, menores niveles de percepción que los chicos; en segundo lugar, que los tipos de beneficios percibidos por chicos y chicas son ligeramente distintos. Las chicas afirman obtener de su ocio, en menor medida 
que los chicos, destrezas y habilidades técnicas. Lo mismo sucede con los beneficios físicos, ambos tipos de beneficios, fuertemente asociados al ocio físico-deportivo. Sin embargo, las puntuaciones medias se equiparan cuando se trata de beneficios emocionales, lo que significa que tanto chicos como chicas disfrutan de un ocio altamente satisfactorio. Estos resultados apuntan, como lo hacen estudios previos (Barnett, 2013; Codina, Pestana, Castillo y Balaguer, 2016), la existencia de diferencias importantes en torno al ocio de chicos y chicas (lo que les resulta más significativo, el tiempo que le dedican, la frecuencia o compañía con la que lo hacen, etc.). No obstante, siguiendo la estela de pensamiento de Henderson (2009), se confirma que para avanzar en la comprensión del papel del género en el ocio juvenil resulta necesario superar el tratamiento habitual de este aspecto (género) como variable independiente y profundizar, desde enfoques más neutros, en los factores implicados en la construcción social del género y, que probablemente, puedan explicar estas diferencias.

Los resultados obtenidos avalan la idea de que ciertos espacios resultan especialmente propicios para el logro de determinado tipo de beneficios, en consonancia con las actividades que acogen. Así los espacios públicos abiertos congregan, especialmente, el ocio físico-deportivo y las fiestas populares. Las plazas, parques y calles son también espacios idóneos para estar con las amistades, una de las actividades de ocio que mejor representa la categoría "Otras actividades". Todo ello explica por qué este tipo de espacios está asociado a beneficios emocionales y sociales. Los espacios cerrados ajenos, tales como polideportivos, gimnasios, cines, ludotecas, centros culturales, discotecas y bares, acogen casi la mitad de las actividades de ocio físico-deportivo juveniles, así como buena parte de sus actividades culturales. De ahí que, además de altos niveles de beneficios emocionales y sociales, estos espacios se muestren asociados a la percepción de beneficios físicos y adquisición de destrezas y habilidades técnicas. Finalmente, los espacios cerrados propios (la casa, locales privados o lonjas), que acogen buena parte de las actividades culturales juveniles y, en menor medida, fiestas o celebraciones de índole privada parecen ser fuente principal de beneficios emocionales y sociales, fundamentalmente. La literatura consultada subraya que la población joven prefiere y se siente más satisfecha en espacios de ocio que le garanticen cierta autonomía del mundo adulto. Los resultados de este estudio coinciden con los ya existentes (AbbotChampan y Robertson, 2001) y apuntan que la satisfacción (beneficios emocionales) de este colectivo es alta en todos los tipos de espacios, no solo en los cerrados particulares (a priori, especialmente proclives a la autogestión), pero además, permiten constatar que estar satisfecho/a con los espacios cerrados 
particulares no implica necesariamente que en ellos se obtengan altos niveles de otros tipos de beneficios. De hecho, este estudio confirma que la mayor percepción de beneficios se da en los espacios cerrados ajenos, en los que se realizan actividades de ocio preferentemente organizado y que es, precisamente, el tipo de ocio que emerge como principal fuente de beneficio.

Efectivamente, el carácter organizado o no organizado del ocio juvenil se revela como una variable para la consecución de beneficios a través del ocio. Más de la mitad (6 de cada 10) de las actividades de ocio significativas para las y los jóvenes españoles son realizadas al margen de cualquier entidad. La importancia de este dato se evidencia al confirmar que la percepción de beneficios es mayor cuando se trata de formas de ocio organizado. Esta diferencia en términos de beneficios a favor del ocio organizado explica por qué las actividades físico-deportivas y el asociacionismo, que son las que más frecuentemente se realizan en un marco organizado, son también las que proporcionan niveles más altos de beneficios, mientras que el ocio digital y las actividades culturales, cuya práctica se realiza en mayor medida, de forma no organizada, son las que muestran medias de beneficios más bajas en todos los tipos de beneficios, excepto en los emocionales. Estos resultados coinciden con los obtenidos en estudios precedentes (Codina, Pestana, Castillo y Balaguer, 2016; Parra, Oliva y Antolín, 2009; Ramos, Rivera y Moreno, 2012) en los que se confirma que el ocio organizado incentiva el desarrollo juvenil por los numerosos beneficios que desencadena (experiencias enriquecedoras, mayor ajuste emocional, desarrollo de la iniciativa personal y cultivo de valores como la responsabilidad, la superación o el respeto a la diversidad) (Mahoney, Larson, Eccles y Lord, 2005; Parra, Oliva y Antolín, 2009; Sibthorp, Bialeschki, Morgan y Browne, 2013). La literatura científica ratifica, por tanto, el interés de que la población joven se implique en prácticas de ocio organizado pues con ello se propicia un mayor logro de beneficios de diversa índole. Sin embargo, estos datos han de interpretarse con cautela, tomando conciencia de que las investigaciones realizadas al respecto se limitan al ocio organizado. No se tiene constancia de estudios que hayan revisado los retornos positivos del ocio juvenil no organizado. En este artículo son las y los propios jóvenes quienes anuncian que su ocio organizado es más fructífero, en términos de beneficios, que el que realizan por su cuenta. Ahora bien, lejos de descartar las bondades del ocio no organizado ante la evidencia de los datos, nos reafirmamos en la necesidad de continuar estudiando el ocio no organizado para esclarecer la naturaleza o condiciones en las que se optimiza la contribución del ocio no organizado al desarrollo juvenil. 


\section{BIBLIOGRAFÍA}

Abbott-Chapman, J. y Robertson, M. (2001). Youth, Leisure and Home: Space, Place and Identity. Loisir et Société /Society and Leisure, 24(2), 485-506.

Ahedo, R. y Macua, A. (2016). Características de las prácticas de ocio físico-deportivas significativas de los jóvenes españoles. Revista de Psicología del Deporte, 25(2), 67-72.

Barnett, L.A. (2013). What people want from their leisure. Journal of Leisure Research, 45 (2), 150-191.

Broh, B. A. (2002). Linking extracurricular programming to academic achievement: Who benefits and why? Sociology of Education, 75, 69-96.

Chawla, L. (2015). Benefits of nature contact for children. Journal of Planning Literature, 30, 433-452.

Codina, N., Pestana, J.V., Castillo, I. y Balaguer, I. (2016). "Ellas a estudiar y bailar, ellos a hacer deporte: Un estudio de las actividades extraescolares de los adolescentes mediante los presupuestos de tiempo. Cuadernos de Psicología del Deporte, 6(1), 233-242.

Colás, P., González, T. y De Pablos, J. (2013). Juventud y redes sociales: motivaciones y usos preferentes. Comunicar, 40 (XX), 15-23.

Cuenca, M. (2015). Ocio valioso. Documentos de Estudios de Ocio, núm. 52. Bilbao: Universidad de Deusto.

Damon, W. (2004). What is positive youth development? Annals of the American Academy of Political and Social Science, 591, 13-24.

Devine, M.A. y Parr, M. (2008). 'Come on in, but not too Far:' Social Capital in an Inclusive Leisure Setting. Leisure Sciences, 30(5), 391-408.

Doistúa, J. y Ried, A. (2016). Ocio en la naturaleza como espacio de desarrollo juvenil. Revista de Psicología del Deporte, 25(3), 39-44.

Driver, B. L. y Bruns, D. H. (1999). Concepts and uses of the benefits approach to leisure. En E. L. Jackson y T.L. Burton, Leisure studies: prospects for the 21st century. State College, PA: Venture Publishing, 349-369.

Driver, B.L., Brown, P.J. y Peterson, G.L. (1991). Benefits of Leisure. State College, PA: Venture Publishing.

Eccles, J. y Gootman, J. (Eds). (2002). Community programs to promote youth development. Washington, D.C.: National Academy Press.

Fraguela, R., Varela, L. y Sanz, E. (2016). Ocio deportivo, imagen corporal y satisfacción vital en jóvenes españoles. Revista de Psicología del Deporte, 25(2), 33-38.

Gardner, M., Roth, J. y Brooks-Gunn, J. (2008). Adolescents' participation in organized activities and developmental success two and eight years after high school: Do sponsorship, duration, and intensity matter?. Developmental Psychology, 44, 814-830.

Gibson, H. y Chang, S. (2013). Cycling in Mid and Later Life: Involvement and Benefits Sought from a Bicycle Tour. Journal of Leisure Research, 44 (1), 23-51.

Henderson, K. A. (2009). Just research and physical activity: Diversity is more than an independent variable. Leisure Sciences, 31(1), 100-105. 
Kleiber, D.A., Bayón, F, Cuenca, J. y Monteagudo, M. J. (2014). La contribución del ocio experiencial valioso al envejecimiento satisfactorio: estudio de los estilos de ocio de las personas mayores de Euskadi. Inguruak: Revista vasca de sociología y ciencia política, 57-58, 2347-2364.

Kleiber, D. A., Walker, G. y Mannell, R. C. (2011). A social psychology of leisure (2nd ed.). State College, PA: Venture Publishing.

Larson R., Jarrett, R., Hansen, D., Pearce, N., Sullivan, P., Walker, K., Watkins, N. y Wood, D. (2004). Organized youth activities as contexts of positive development. En P. A. Linley y S. Joseph (eds.) Positive psychology in practice. New York: John Wiley \& Sons, 540-560.

Larson, R. W. (2000). Toward a psychology of positive youth development. The American Psychologist, 55, 170-183.

Mahoney, J. L., Larson, R. W. y Eccles, J. S. (2005). Organized activities as contexts of development: Extracurricular activities, after-school and community programs. Hillsdale, NJ: Lawrence Erlbaum Associates.

Mahoney, J. L., Larson, R. W., Eccles, J. S. y Lord, D. (2005). Organized activities as developmental context for children and adolescents. En J. L. Mahoney, R. W. Larson y J. S. Eccles (Eds.). Organized activities as a contexts of development: Extracurricular activities, after-school and community programs. Hillsdale, NJ: Lawrence Erlbaum Associates, 3-22.

Monteagudo, M.J. (2004). Los beneficios del ocio: qué son y para qué sirven. ADOZ. Revista de Estudios de Ocio, 28,5563-72.

Monteagudo, M. J. (2008). Reconstruyendo la experiencia de ocio: características, condiciones de posibilidad y amenazas en la sociedad de consumo. En M. J. Monteagudo (ed.) La experiencia de ocio: una mirada científica desde los Estudios de Ocio. Bilbao: Universidad de Deusto, 81-110.

Monteagudo, M. J., Ahedo, R., Richter, F. y Doistua, J. (2014). Repensando el deporte escolar desde nuevos parámetros. En C. Ortega y F. Bayón (coords.) El papel del ocio en la construcción social del joven. Bilbao: Universidad de Deusto, 197-209.

Nussbaum, M. (2012). Crear capacidades: propuesta para el desarrollo humano. Barcelona: Paidós.

Oliva, A., Pertegal, M.A., Antolín, L., Reina, MC. y Ríos, M. (2011). El desarrollo positivo adolescente y los activos que lo promueven. Un estudio en centros docentes andaluces. Sevilla: Junta de Andalucía. Consejería de Salud.

Ortega, C., Lazcano, I. y Baptista, M. (2015). Espacios de ocio para jóvenes, de la monitorización a la autogestión. Pedagogía Social, Revista Interuniversitaria, 25, 69-89.

Parra, A., Oliva, A. y Antolín, L. (2009). Los programas extraescolares como recurso para fomentar el desarrollo positivo adolescente. Papeles del Psicólogo, 30 (3), 265-275.

Ponce de León, A., Sanz, E., y Valdemoros, M. Á. (2015). Ocio familiar y actividad física en estudiantes de bachillerato: ¿Alianza, rivalidad o independencia? Pedagogía Social. Revista Interuniversitaria, 25, 51-68.

Porter H., Iwasaki Y. y Shank J. (2010). Conceptualizing meaning-making through leisure experiences. Society and Leisure/Loisir et Societe, 33(2), 167-194. 
Ramos, P., Rivera, F. y Moreno, C. (2012). Beneficios del contexto asociativo en las actividades de tiempo libre de los adolescentes españoles. Infancia y Aprendizaje, 35(3), 365-378.

Roth, J. L. y Brooks-Gunn, J. (2003). What exactly is a youth development program? Answers from research and practice. Applied Developmental Science, 7, 94-111.

Sibthorp, J., Bialeschki, M.D., Morgan, C. y Browne, L. (2013). Validating, norming, and utility of a youth outcomes battery for recreation programs and camps. Journal of Leisure Research, 45(4), 514-536.

Tejerina, B., Carbajo, D. y Martínez, M. (2012). El fenómeno de las lonjas juveniles. Nuevos espacios de ocio y socialidad en Vitoria-Gasteiz. Informes del CEIC, 004.

Valdemoros, M, A., Ponce de León, A. y Gradaille, R. (2016). Actividad Física de Ocio Juvenil y Desarrollo Humano. Revista de Psicología del Deporte, 25(2), 45-51.

Viñals, A. y Cuenca, J. (2016) Ocio entre pares en la era digital: percepción del ocio conectado juvenil. Revista de Psicología del Deporte, 25(2), 61-65.

Zarret, N., Fay, K., Li, Y., Carrano, J., Phelps, E. y Letner, R. M. (2009). More than child's play: variable and pattern-centered approaches for examining effects of sports participation on youth development. Development Psychology, 45, 368-382.

Zeijl, E., Du Bois-Reymond, M. y Te Poel, Y. (2001). Young Adolescents' Leisure Patterns, Society and Leisure, 24(2), 379-402.

MARÍA JESÚS MONTEAGUDO SÁNCHEZ. Doctora en Ocio y Potencial Humano y Licenciada en Psicología por la Universidad de Deusto. Investigadora asociada en el Instituto de Estudios de Ocio y miembro del equipo de Investigación "Ocio y Desarrollo Humano" de la Universidad de Deusto, reconocido por Gobierno Vasco en categoría A (IT984-16). Su investigación se centra en el estudio del ocio como factor de desarrollo humano, el ocio a lo largo de la vida, beneficios y barreras para la participación así como en el ocio deportivo. Es autora de una decena de monografías, más de 25 capítulos de libro y casi una veintena de artículos en revistas especializadas de alto impacto. Ha sido IP de casi una veintena de investigaciones y ha participado como investigadora en 36 proyectos estatales e internacionales.

orcid.org/0000-0002-6725-5265. Email: mjmonte@deusto.es Correspondencia: María Jesús Monteagudo. Avda. universidades 24, 48007 Bilbao. Bizkaia. España.

RUTH AHEDO GONZÁLEZ: Licenciada en Psicología por la Universidad de Deusto y Doctoranda en el programa Ocio, Cultura y Comunicación para el Desarrollo Humano. Es miembro del Instituto de Estudios de Ocio y forma parte del equipo de Investigación Ocio y Desarrollo Humano de la Universidad de 
Deusto. Vinculada a este equipo ha desarrollado numerosas investigaciones, entre ellas, los Planes Nacionales RESORTES (2013-2015) e ITINERE (20132015), así como el proyecto europeo CONNECT. Connecting Audiences (20172019). orcid.org/0000-0002-4416-5187. Email: ruth.ahedo@deusto.es

ANA PONCE DE LEÓN ELIZONDO: Catedrática de la Universidad de La Rioja. Doctora en Ciencias de la Educación, Premio Extraordinario Doctorado (1996). Directora del Grupo de Investigación Actividad Física y Deporte en el espacio y tiempo de Ocio [AFYDO] de la UR y miembro fundador de la Red de Investigación OcioGune. Ha sido Investigadora Principal en 19 proyectos de I+D+I en temas de Educación, Ocio, Actividad Físico-deportiva y Familia; ha dirigido 11 tesis doctorales y es autora de más de un centenar de libros, capítulos de libro y artículos científicos de alto prestigio e impacto internacional integrados en sistemas de indización internacionales como Social Science Citation Index, JCR, Scopus, Erih, Inrecs, Isoc, Latindex, Dice, Recyt, Redalic. orcid.org/0000-0003-4622-8062. Email: ana.ponce@unirioja.es

Recibido: 03/04/2017

Aceptado: 16/09/2017 


\section{BUENAS PRÁCTICAS DE INTERVENCIÓN SOCIAL EN RELACIÓN AL OCIO CON JÓVENES EN RIESGO DE EXCLUSIÓN DESDE LA PERCEPCIÓN DE LOS AGENTES SOCIALES \\ GOOD PRACTICES OF SOCIAL INTERVENTION IN RELATION TO LEISURE WITH YOUNG PEOPLE AT RISK OF EXCLUSION FROM THE PERCEPTION OF SOCIAL AGENTS}

Fátima Poza-Vilches

Facultad de Ciencias de la Educación Universidad de Granada, España fatimapoza@ugr.es

Ana Fernández-García Facultad de Educación Universidad Nacional de Educación a Distancia (UNED), España anafernandez@edu.uned.es

João Paulo Ferreira Delgado Instituto Politécnico do Porto, Portugal pdelgado@ese.ipp.pt

Cómo citar / Citation

Poza-Vilches, Fátima; Fernández-García, Ana y Ferreira Delgado, João Paulo (2017) "Buenas prácticas de intervención social en relación al ocio con jóvenes en riesgo de exclusión desde la percepción de los agentes sociales". OBETS. Revista de Ciencias Sociales, 12(Extra 1): pp-pp. 203-228.

doi:10.14198/OBETS2017.12.1.18

\section{Resumen}

Este artículo plantea delimitar buenas prácticas en el trabajo con jóvenes socialmente vulnerables desde la caracterización de la intervención profesional, en materia de ocio. Para ello se plantea un estudio descriptivo exploratorio de carácter cualitativo a partir del diseño de un cuestionario abierto con 
el que se recoge información sobre la intervención que 34 profesionales vinculados a este campo diseñan y desarrollan.

Bajo este planteamiento la investigación aquí recogida, pretende el cumplimiento de dos objetivos clave: identificar las confluencias y divergencias en la práctica profesional y establecer un protocolo de indicadores que nos ayude, posteriormente, a identificar buenas prácticas de intervención en ocio juvenil.

Palabras clave: Estudio Descriptivo Cualitativo; Buenas Prácticas; Ocio Juvenil; Indicadores; Riesgo de exclusión social.

\section{Abstract}

This article proposes to delimit good practices in the work with socially vulnerable young people from the characterization of the professional intervention, in the matter of leisure. For this purpose, an exploratory descriptive study of a qualitative nature is proposed, based on the design of an open questionnaire that collects information about the intervention that 34 professionals related to this field design and develop.

Under this approach, the research gathered here aims to fulfill two key objectives: to identify the confluences and divergences in professional practice and to establish a protocol of indicators that will help us, later, to identify good intervention practices in youth leisure.

Key words: Qualitative Descriptive Study; Good Practices; Leisure; Youthful; Indicators; Risk of social exclusion.

\section{Extended Abstract}

This article has as main objective to delimit good practices in the work with socially vulnerable young people from the characterization of the professional intervention, in matter of leisure. For this purpose, a qualitative descriptive study is proposed based on the design of an open questionnaire that collects information about the intervention that 34 professionals linked to this field design and develop. The research will allow us to identify the confluences and divergences in professional practice in order to establish a protocol of indicators that will help us, later, to identify good practices of intervention in youth leisure.

This article aims to define good practices in the work with young people in leisure since the characterization of professional intervention. To this end, thirty-four existing experiences are collected at the national level that serve as references to design a system of framework indicators that makes it possible to validate these practices and identify others.

Based on these 34 experiences, we intend to answer questions such as: what objectives are prioritized in professional practice with youth and leisure? What are the methodological and coordination strategies in this field? Are the financial resources used the most useful and efficient to deal with these professional practices? What communication and dissemination strategies do these professionals put in place to reach the target population of the actions? And finally, what evaluation system do they design to monitor and control the achievements. 
In order to answer these questions it is opportune to identify the frame of reference in which we are located. In this sense, in order to favor an improvement of youth leisure and to enable strategies that minimize the effects of a consumption of unhealthy leisure in young people, it is a priority to identify the actions that are developed in this field; To establish strengths and weaknesses, and to identify action strategies for improvement (Pérez-Serrano, PozaVilches \& Fernández-García, 2016, Delgado, Pose \& De Valenzuela, 2015). Likewise, it is fundamental to propose effective intervention lines that counteract the effects of a non-responsible and healthy leisure consumption (Cuenca, 2004; 2009); (Fernández-García \& Poza-Garcia, 1999). Therefore, from the agents involved in this change of behavior, such as families, social agents and youth themselves, certain strategies of action are proposed that can be a starting point to consolidate viable and fruitful proposals Fernández-García, Poza-Vilches \& Fiorucci, 2015; Giménez, Cortés \& Espejo, 2010). Detecting mentioned action strategies and be able to characterize these professionals practices is a priority in this article.

The methodological model from which we started focuses on the diagnostic evaluation of an exploratory cut that starts from the case series of 34 intervention experiences carried out in the national territory; giving prominence to the professional action and the work with young people in the matter of leisure. Identifying the lines of work that help define the complex and dynamic reality of youth and establish guidelines that make it possible to act with it, for the inclusion and integral development of this group; Has been the main reference in this study.

Regarding the strategy that has been considered most opportune to collect the information on the professional practice of the respondents -due to the fact that it is an exploratory pilot study-, it has been the open questionnaire. This questionnaire is the best tool to leave professionals free in their answers (Bisquerra, 2009) and as a pilot study is the starting point that will help us to delimit similar cases of intervention as well as the discrepancies that will enable us to establish an intervention map general in working with young people in leisure.

This questionnaire has been structured into two blocks that have clearly delineated the information collected:

Independent variables: sex, age, maximum qualification, autonomous community where their develop their professional practice, years of experience, employment status and position they currently occupy.

Dependent variables: six open questions that refer to the strategies and actions that seek to define the professional practice of the experts surveyed, delimited by the following dimensions: objectives, methodology, inter- and intrainstitutional coordination, funding sources, communication and dissemination strategy and evaluation system.

Regarding the sample under study, there were 34 experiences analyzed from the opinion of the social agents that execute them and representing eight autonomous communities of the national territory: Community of Madrid (30.3\%); Basque Country (24.2\%); Castilla y León (18.2\%); Andalusia (15.2\%) and Galicia, Aragon and Asturias with 3\% representation respectively. 
Regarding the gender of the respondents, $59 \%$ of the sample were male and $41 \%$ female.

The experiences analyzed revolve around the different areas of development of the youth group, especially at risk of exclusion. These areas of management have to do with: public youth policies in the areas of leisure, training, housing and employment; coordinated management actions in the field of social services; specialized youth work and disability; innovative experiences in education, both at the secondary level, vocational training centers and universities; actions of supervised management of juvenile centers, juvenile immigration and social integration as well as intervention projects in the field of youth health and drug addiction.

Regarding the process that has been followed in the analysis of the information follows, in general terms, that established in the analysis of content of qualitative information.

This study, as a summary, seen from the professional practice and as it appears from the results and conclusions, emanates a system of indicators that seeks to validate in order to identify good practices in the intervention with young people at risk of exclusion and leaving in evidence a great challenge. The challenge is to create structures of interprofessional work that favor the emergence of contextualized actions, linked to the youth needs and emanating from the involvement of all the actors involved, especially from the active participation of the youth group.

In this process of action, the design of networks of communication, training, diffusion and communication is key to itself as the endowment of resources that facilitate the development of the designed.

From here, the monitoring and evaluation system that is profiled will be key to improving the process from the beginning; A premise that must guide professional practice in the field of leisure.

The professionals surveyed, therefore, are invited to become aware of the philosophy of work that must prevail in social institutions that direct their practice to young people and leisure to, from this, be able to create coordinated structures of action, strengthen the network of professionals and provide the resources that abide and prioritize these actions in order to meet a common goal, improve the quality of life of the youth group and promote their integral and social development.

\section{INTRODUCCIÓN}

El presente artículo tiene como objetivo delimitar buenas prácticas en el trabajo con jóvenes en materia de ocio desde la caracterización de la intervención profesional. Para ello se recogen treinta y cuatro (34) experiencias existentes a nivel nacional que sirvan como referentes para diseñar un sistema de indicadores marco que posibilite validar dichas prácticas e identificar otras.

Partiendo de estas 34 experiencias, pretendemos dar respuesta a cuestiones como: ¿qué objetivos se priorizan en la práctica profesional con jóvenes y el 
ocio? ¿cuáles son las estrategias metodológicas y de coordinación referentes en este campo de trabajo? ¿los recursos financieros utilizados son los más útiles y eficientes para poder afrontar estas prácticas profesionales? ¿qué estrategias de comunicación y difusión ponen en marcha estos profesionales para llegar a la población destinataria de las acciones? Y finalmente, ¿qué sistema de evaluación diseñan para hacer un seguimiento y control de los logros obtenidos?

Para dar respuesta a estas cuestiones es oportuno previamente identificar el marco de referencia en el que nos situamos. En este sentido, para favorecer una mejora del ocio juvenil y posibilitar estrategias que minimicen los efectos de un consumo de ocio no saludable en jóvenes, es prioritario identificar las acciones que se desarrollan en este campo; establecer puntos fuertes y débiles e identificar estrategias de acción para la mejora (Pérez-Serrano, PozaVilches y Fernández-García, 2016; Delgado, Pose y De Valenzuela, 2015). Asimismo, es fundamental plantear líneas de intervención eficaces que contrarresten los efectos de un consumo de ocio no responsable y saludable (Cuenca, 2004; 2009); por ello, desde los agentes implicados en este cambio de comportamientos, como son familias, agentes sociales y la propia juventud, se plantean ciertas estrategias de acción que pueden ser un punto de partida para consolidar propuestas viables y fructíferas (Fernández-García, Poza-Vilches y Fiorucci, 2015; Giménez, Cortés y Espejo, 2010). Detectar dichas estrategias de acción y poder caracterizar estas prácticas profesionales es una prioridad en este artículo.

En la práctica profesional con jóvenes en materia de ocio y, especialmente en riesgo de exclusión social; entendiendo a este colectivo como aquellos que se encuentran en situaciones de mayor desigualdad y muestran rasgos de exclusión social en su tránsito a la vida adulta (Kovacheva, 2007; Stebbins, 2012); autores como Birkeland, Torsheim, \& Wold (2009); Pérez-de-Guzmán y Trujillo (2011) o Vidarte y Vélez (2012), ponen de manifiesto la necesidad de trabajar con metodologías de trabajo activas y participativas que partan de las necesidades reales juveniles y que se creen proyectos desde su propia realidad; proyectos para, con, desde y por la juventud. Esto es una cuestión fundamental para que la participación juvenil sea real y efectiva y además, como señalan los autores anteriores, desde la inclusión del colectivo juvenil en este tipo de dinámicas y en este tipo de actividades, se puede crear una correlación positiva en relación a la potenciación de hábitos y actitudes juveniles proactivas que beneficien incluso, el estado de ánimo de este colectivo.

Es fundamental no partir de una intervención fragmentada y focalizada sino desde una acción social que movilice escenarios de trabajo compartidos 
y que promueva procesos de inclusión juvenil intentando responder a necesidades sociales y, específicamente, incidir significativamente en la interacción de las personas, aspirando a una legitimación pública o social (Paz y Unás, 2010; Fantova, 2007). La cultura del ocio presupone un conjunto de valores y de prácticas que deben realizarse "para los ciudadanos y ciudadanas, e inevitablemente con ellos" (Caride, 2009:172).

Desde este marco de integralidad de las acciones, el estudio que aquí se detalla pretende visibilizar la labor que se desarrolla con jóvenes en riesgo de exclusión social y analizar su filosofía de acción que nos ayude, por tanto, a caracterizar la práctica profesional con jóvenes vulnerables socialmente para su desarrollo integral e inclusión social.

Realizar un estudio diagnóstico y exploratorio para evaluar la práctica profesional en el trabajo con jóvenes y ocio, es el eje central de este trabajo que contribuirá a identificar desde la perspectiva profesional,tanto los aspectos positivos como lo negativos de toda intervención siempre con el objetivo de orientar hacia la mejora, dicha práctica.

\section{MARCO METODOLÓGICO}

\section{Modelo metodológico}

El modelo metodológico del que partimos se centra en la evaluación diagnóstica de corte exploratorio que parte de la casuística de 34 experiencias de intervención llevadas a cabo en el territorio nacional; dando protagonismo a la acción profesional y al trabajo con jóvenes en materia de ocio. Identificar las líneas de trabajo que ayudan a definir la realidad compleja y dinámica de la juventud y establecer pautas que posibiliten actuar con aquella, para la inclusión y desarrollo integral de este colectivo; ha sido el referente primordial en este estudio. Marí (2007) señala de hecho la prioridad de trabajar desde modelos diagnósticos participativos:

Existe un consenso entre los profesionales y especialistas en que la realidad socioeducativa exige una actividad diagnóstica centrada en el desarrollo personal y en las competencias como estructuras dinámicas con una finalidad de cambio y mejora. Esta realidad, por supuesto, comporta dos objetivos evidentes, por una parte, abandonar los modelos diagnósticos tradicionales centrados en las deficiencias y, por otra parte, adoptar una visión diagnóstica más amplia que contemple el proceso de aprendizaje y de crecimiento continuado del sujeto o del grupo, desde la dinámica y la complejidad del objeto de estudio (Marí 2007:612). 


\section{Estrategias de recogida de información}

Respecto a la estrategia que se ha considerado más oportuna para recoger la información sobre la práctica profesional de los encuestados -atendiendo a que es un estudio piloto de corte exploratorio-, ha sido el cuestionario abierto. Dicho cuestionario es la mejor herramienta para dejar libertad a los profesionales en sus respuestas (Bisquerra, 2009) y como estudio piloto es el punto de partida que nos ayudará a delimitar casuísticas de intervención similares así como las discrepancias que nos posibilitarán establecer un mapa de intervención general en el trabajo con jóvenes en materia de ocio.

Dicho cuestionario se ha estructurado en dos bloques que han delimitado claramente la información recogida:

\begin{tabular}{l|l}
\hline Variables & Sexo \\
Independientes & Edad \\
& $\begin{array}{l}\text { Titulación máxima } \\
\text { Comunidad autónoma donde desarrolla su práctica profesional } \\
\text { Años de experiencia } \\
\text { Situación laboral } \\
\text { Puesto que ocupa actualmente }\end{array}$ \\
\hline Variables & $\begin{array}{l}\text { Seis cuestiones abiertas que aluden a las estrategias y acciones } \\
\text { que pretenden definir la práctica profesional de los expertos } \\
\text { encuestados, delimitadas por las siguientes dimensiones: } \\
\quad \text { Objetivos } \\
\text { Metodología } \\
\text { Coordinación inter e intra institucional } \\
\text { Fuentes de financiación } \\
\text { Estrategia de comunicación y difusión } \\
\text { Sistema de evaluación }\end{array}$ \\
\hline
\end{tabular}

\section{Muestra}

Respecto a la muestra objeto de estudio, han sido 34 las experiencias analizadas desde la opinión de los agentes sociales que las ejecutan y con representación de ocho comunidades autónomas del territorio nacional: Comunidad de Madrid (30,3\%); País Vasco (24,2\%); Castilla y León (18,2\%); Andalucía (15,2\%) y Galicia, Aragón, Murcia y Asturias con un 3\% de representatividad respectivamente.

En cuanto al sexo de los encuestados, el 59\% de la muestra han sido hombres y el $41 \%$ mujeres.

Las edades oscilan entre los 46 y 50 años en el 32,4\% de la muestra; entre los 41 a 45 años en el 20,6\%; entre 51 y 55 años hay una representación del 
14,7\%; el 11,8\% oscila entre los 26 y 35 años y solo un 5,9\% se agrupa entre los 36 y 40 años.

La titulación ${ }^{1}$ de referencia es variada aunque el 41,2\% son licenciados; el 29,4\% tiene formación de máster; el 11,8\% tienen diplomatura y solo un 8,8\% tienen el título de doctor o Formación Profesional de II Grado.

El bagaje laboral de estos encuestados es extenso en la mayoría ya que el $37,5 \%$ tiene más de 21 años de experiencia; el $28,1 \%$ tiene una vida profesional que oscila entre los 6 y 10 años; entre 16 y 20 años se sitúa el 18,8\% de la muestra y entre 11 y 15 , el 15,6\% de los encuestados.

Finalmente, respecto a la práctica profesional, el 39,4\% la desarrolla en Entidades Sociales (tales como asociaciones, fundaciones, y ONG's); el 36,4\% su vinculación laboral está asociada a la Administración Pública (como entidades locales, diputaciones provinciales, centros de menores, centros educativos o universidades) y para el $15,2 \%$ su referente laboral es la empresa privada (centros de formación especializada o centros de ocio juvenil). Asimismo, la vinculación laboral de un $9 \%$ de los expertos está unida tanto a la empresa privada y pública o incluso vinculada a las entidades sociales y con labores de voluntariado.

Las experiencias analizadas giran en torno a los diferentes ámbitos de desarrollo del colectivo juvenil, especialmente en riesgo de exclusión. Estos ámbitos de gestión tienen que ver con: políticas públicas de juventud en materia de ocio, formación, vivienda y empleo; acciones de gestión coordinada en el ámbito de los servicios sociales; trabajos especializados en jóvenes y discapacidad; experiencias innovadoras en el ámbito educativo tanto a nivel de secundaria, centros de formación profesional como universidad; acciones de gestión tutelada de centros de menores, inmigración juvenil y de integración social así como proyectos de intervención en el ámbito de la salud juvenil y drogadicción.

En líneas generales las áreas de trabajo de los 34 encuestados se especifican en tres perfiles tipo: técnicos y profesionales con puestos base en el campo de la educación social, que representan un 32,35\% de los participantes; profesorado y expertos del campo en el ámbito de la formación (educación secundaria, formación profesional y universidad), un 29,41\% del total y, especialistas en la gestión (puestos de coordinación, supervisión y dirección en este campo), un 38,24\%.

El tipo de muestreo utilizado ha sido de corte no probabilístico por conveniencia ya que se solicitó a profesionales y expertos que cumplían la característica de tener información relevante sobre el objeto de estudio por su formación, puesto de trabajo y experiencia, que participaran en la investigación.

${ }^{1}$ El ítem de la formación se presentó como pregunta de respuesta abierta por lo que los participantes del estudio especificaron claramente su formación de base y en ningún caso ha sido la de poseer el título de graduado. Entendemos que este hecho ocurre dada la edad de la mayoría de las personas participantes en el estudio. 


\section{PROCEDIMIENTO DE ANÁLISIS DE LA INFORMACIÓN}

El proceso que se ha seguido en el análisis de la información sigue, en líneas generales, el establecido en el análisis de contenido de información cualitativa, identificada por las siguientes fases:

\begin{tabular}{l|l}
\hline ETAPAS & DESCRIPCIÓN \\
\hline la información & $\begin{array}{l}\text { Una vez recogida la información se han ido transcribiendo } \\
\text { todos los textos y almacenándolos en ficheros RTF para que } \\
\text { el formato fuera compatible con el programa de análisis cua- } \\
\text { litativo Atlas ti. }\end{array}$ \\
\hline $\begin{array}{l}\text { 2. Tratamiento de } \\
\text { los datos en el } \\
\text { programa Atlas.ti }\end{array}$ & $\begin{array}{l}\text { El primer tratamiento de los datos ha consistido en numerar } \\
\text { las narraciones siguiendo la vía de numeración de párrafos. } \\
\text { Se ha llevado a cabo una primera lectura iniciando un ciclo } \\
\text { de interpretación significativa de toda la transcripción. En } \\
\text { una segunda lectura, hemos comenzado un proceso de cate- } \\
\text { gorización a partir de la selección de los fragmentos de texto } \\
\text { según los parámetros temáticos definidos en las categorías. } \\
\text { El cruce de esta información con las anotaciones espontáneas } \\
\text { realizadas en la primera lectura, ha permitido ir modificando } \\
\text { y puliendo la parrilla de categorías progresivamente. }\end{array}$ \\
\hline $\begin{array}{l}\text { 3. Codificación de } \\
\text { la información }\end{array}$ & $\begin{array}{l}\text { Hemos simultaneado los dos procedimientos de codificación: } \\
\text { la codificación rápida, cuando los fragmentos de texto repre- } \\
\text { sentaban una única categoría íntegramente y la codificación } \\
\text { manual, cuando en un mismo párrafo aparecían diferentes } \\
\text { categorías que había que marcar dejando parte del párrafo } \\
\text { fuera de la selección. }\end{array}$ \\
\hline la información & $\begin{array}{l}\text { Una vez finalizada la etapa anterior hemos solicitado el primer } \\
\text { informe codificado y a partir de éste, el programa nos ofrece } \\
\text { una versión ordenada de los textos por metacategorías (árbo- } \\
\text { les) y nudos (categorías temáticas). Sobre este fichero hemos } \\
\text { iniciado nuestro volcado de información a las diferentes cate- } \\
\text { gorías planteadas. }\end{array}$ \\
\hline
\end{tabular}

La estrategia empleada para el análisis de la información recogida de corte cualitativo ha sido el "análisis de contenido". Siguiendo a Poza-Vilches (2008) con esta estrategia hemos pretendido analizar no tanto el estilo del texto sino las ideas expresadas en él; en este caso hemos tenido en cuenta las percepciones que los profesionales han manifestado respecto a su práctica profesional, para conocer la tendencia e incluso llegar a identificar posibles patrones de intervención. 
El análisis de contenido, en palabras de López Noguero (2002:132); "se sitúa en el ámbito de la investigación descriptiva y pretende sobre todo, descubrir los componentes básicos de un fenómeno determinado extrayéndolos de un contenido dado a través de un proceso que se caracteriza por el intento de rigor de medición".

La categorización general de análisis de la información de la que partimos está formada por los siguientes elementos: objetivos, metodología, coordinación intra e inter institucional, fuentes de financiación, estrategias de comunicación y difusión y sistema de evaluación.

\section{RESULTADOS}

Seguidamente se exponen los resultados obtenidos tras el análisis de la información recogida de los informantes clave de la presente investigación.

\section{Objetivos}

La primera red que se presenta se corresponde con los objetivos más relevantes que, en materia de ocio, los expertos encuestados han destacado como fundamentales para una intervención social eficaz con jóvenes.

Figura 1. Mapa conceptual análisis cualitativo. Red: Objetivos

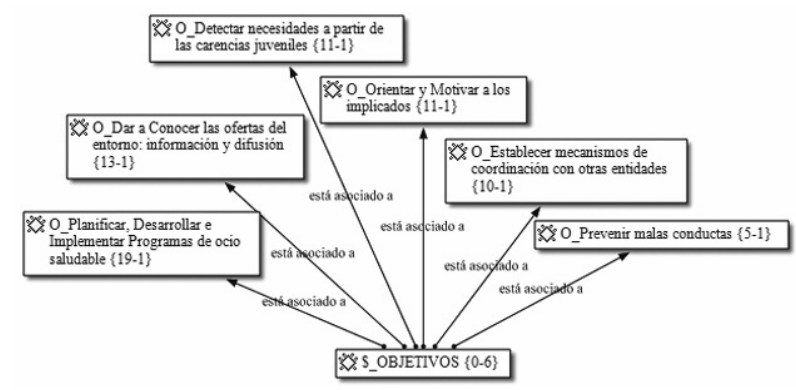

El 56\% de los expertos encuestados (19 personas de los 34 participantes) han declarado que el objetivo más importante es la implementación de los programas de ocio que se desarrollan en las instituciones. Se defiende un tiempo de ocio en la vida de los jóvenes en el que las actividades de ocio ocupen un lugar protagonista. Dichas actividades tienen que ver con salidas extraescolares, deporte, actividades psicoeducativas, etc.; ofertando propuestas educativas que se adapten a sus tiempos con la finalidad de que conozcan contextos diferentes, potenciando hábitos saludables y descontextualizando a los jóvenes de la cotidianeidad de la vida diaria, entre otros. 
Asimismo, estos expertos exponen que es fundamental crear inquietudes entre los jóvenes dando otro tipo de opciones para ocupar su tiempo libre, por ejemplo formando en comunicación y responsabilidad; fomentando el trabajo en valores y la igualdad de oportunidades así como en resolución, gestión de conflictos y visión de futuro; considerando el tiempo de ocio como un espacio de aprendizaje, creatividad y de diversión. Todo ello favoreciendo la integración social de las personas a través de la puesta en práctica de actividades de ocio saludables.

El 38\% de los profesionales encuestados (13 participantes de los 34 encuestados) han afirmado que un objetivo fundamental es informar y difundir todas las actuaciones de interés a nivel de ocio, disponibles en el entorno más próximo de los jóvenes poniendo a su disposición información de recursos o centros especiales de ocio juvenil del barrio o zona donde residen (centros juveniles, oficinas de información juvenil, entidades municipales, asociaciones de barrio, centros concertados o privados, centros cívicos...). Con ello piensan estos expertos que puede acercar al joven a su ciudad y sus recursos, tales como el patrimonio cultural y medioambiental que ofrece.

De este modo lo han expresado lo expertos encuestados:

"Ayudar en la búsqueda de actividades de ocio que sean solicitadas por el joven, informar sobre las entidades cercanas, y si fuera necesario el acompañamiento al recurso. Del mismo modo, nos aseguramos de realizar un seguimiento de la adaptación del joven a la actividad valorando su grado de satisfacción y la continuidad en la misma" (Encuestado 4. E4).

En tercer lugar, el 32\% de los encuestados (11 participantes) ha declarado que la detención de necesidades a partir de las carencias juveniles es un objetivo primordial. Para ello se llevan a cabo distintas estrategias como pueden ser la consulta a los jóvenes, incluyendo a padres y profesorado, es decir, individualmente o en grupo. Respecto a los jóvenes, es necesario hacer una evaluación individual de cada caso y sondear sus intereses reales, así como su disposición para realizar las actividades de ocio.

Proponen que se ha de partir de las propuestas de los propios jóvenes y en función de sus intereses se han de diseñar las futuras actividades que se realizarán, dotándolas (transversalmente) de una alta carga educativa.

Otro aspecto importante es innovar de manera continuada en los programas de actividades, adaptados a las necesidades observadas y creando alternativas frente a las dificultades que entorpecen el crecimiento y desarrollo personal de los jóvenes. Para ello es importante analizar de manera continuada la realidad y el entorno de los jóvenes en dificultad social, facilitando equipamientos y recursos adecuados y variados en función de necesidades detecta- 
das y demandas recogidas. También han resaltado como relevante, la detección precoz de problemas, que pueden encontrarse en investigaciones y estudios.

Por otro lado, el 32\% de los expertos entrevistados (11 participantes) han destacado como un objetivo relevante el orientar y motivar a los jóvenes mediante diferentes acciones como el apoyo que se puede ofrecer a los jóvenes en materia educativa, tales como la ayuda en aquellas materias académicas que lo necesiten.

Asimismo, un elemento muy importante es motivar y fomentar actitudes y cauces de participación en propuestas de ocio y tiempo libre, sobretodo en el disfrute de un ocio saludable.

"Si trabajamos en materia de ocio juvenil, los que tienen que participar de forma activa en esto son los jóvenes y a ellos debemos dirigir muchas de nuestras preguntas" (E12).

Finamente, se destaca que los jóvenes deben entender el ocio como una parte de la vida de las personas, que nos ayuda a fomentar nuestra identidad y es un elemento primordial, no secundario.

El 29\% de los expertos encuestados (10 participantes) han declarado como otro importante objetivo la coordinación que se establece entre los profesionales en materia de ocio (entidades públicas, privadas, empresas...), fomentando el trabajo en equipo e iniciativas de acción conjunta. Es fundamental conocer la oferta del entorno, mediante la utilización de recursos comunitarios. Igualmente relevante es completar el trabajo realizado por las instituciones en formación.

Finalmente, el 15\% de los profesionales entrevistados (5 participantes) han destacado como un objetivo relevante la prevención de conductas de riesgo. Estas intervenciones pueden realizarse mediante actividades extraescolares o acciones informativas desde asociaciones que trabajan con jóvenes en el desarrollo de hábitos saludables y, más específicamente, con tratamiento ambulatorio o comunidades terapéuticas para la prevención de drogodependencias, por ejemplo. Un elemento destacado es promover una toma de conciencia crítica y sensible hacia nuestro entorno cultural y medioambiental.

\section{Metodología}

Con relación a la metodología, se presenta una red definida por siete características que definen el marco de actuación más idóneo para la intervención con jóvenes.

El 32,3\% de los profesionales entrevistados (11 participantes) defienden una metodología comunicativa, basada en el intercambio y la comunicación inter- 
Figura 2. Mapa conceptual análisis cualitativo. Red: Metodología

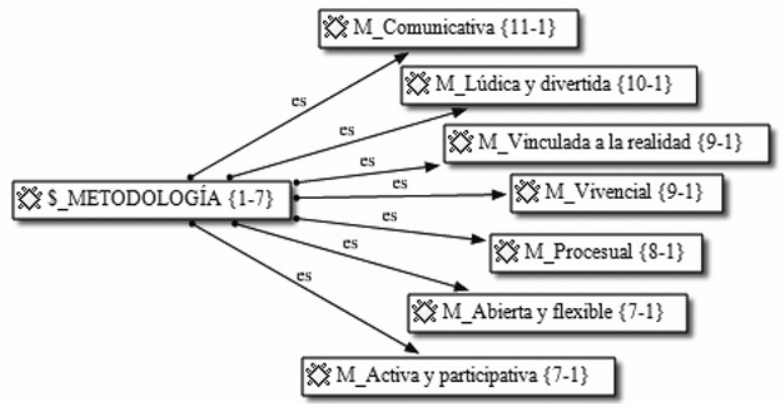

personal en todas sus formas y lenguajes. Reiteran que es imprescindible abrir todos los canales de comunicación posibles: escuchando, respetando y teniendo en cuenta la opinión de todos los implicados.

Para ello se torna necesario valorar las características de las generaciones más jóvenes, eliminando prejuicios tal y como se expone:

"Los "milleniards" tienen mucho que aportar, pero entre el profesorado todavía pesa mucho la opinión de que son vagos, con todo resuelto y sin aspiraciones" (E2).

Varios de los expertos encuestados han citado la importancia de la función de las personas significativas "como acompañantes" (personal de las instituciones) de los jóvenes, para apoyarles y motivarles.

Asimismo se reitera la necesaria participación de padres y madres en las actividades de ocio juvenil, creando espacios de trabajo y reflexión y estableciendo relaciones horizontales con los jóvenes.

Por su parte, el 29,4\% de los encuestados (10 participantes) defienden una metodología lúdica y divertida. Entre las características de esta metodología destacan basar las actividades en el juego, conocimiento y creatividad. Es importante fomentar la motivación y sensibilización para la participación, tanto de los jóvenes como de los profesionales.

Los expertos reiteran la necesidad de programar actividades atractivas para llegar a la mayoría. Ello se recoge en la siguiente información ofrecida por un profesional encuestado:

"Es importante que los jóvenes se sientan motivados y que la oferta sea lo suficientemente atractiva que satisfaga sus expectativas y necesidades. El ocio debe intentar conjugar aspectos tales como: el divertimento, la relaciones sociales, el desarrollo personal, etc." (E29). 
Respecto a los jóvenes en dificultad social, es importante trabajar la motivación para conocer y experimentar otro tipo de actividades saludables que normalmente, por su entorno familiar y social, no suelen realizar (teatro, salidas al aire libre, deporte, exposiciones, cine...).

Asimismo, el 26,4\% (9 participantes) de los expertos encuestados considera muy relevante una metodología vinculada a la realidad, que parta del conocimiento y análisis de la situación concreta de los participantes.

Los profesionales creen necesario la existencia de una oferta de ocio individualizada que favorezca, que en un mismo periodo de tiempo, se realicen actividades que permitan integrar a cada persona en la actividad que mejor se adecúe a sus necesidades, intereses y capacidades.

A nivel práctico, estos expertos consideran que la estrategia metodológica consiste en convertir a cada joven en protagonista de su proceso, escuchando sus inquietudes e intentando que le dediquen tiempo y esfuerzo a esa inquietud. Los profesionales ayudan a los jóvenes a encontrar los espacios adecuados para su desarrollo, se les acompaña en las gestiones oportunas y si es necesario se trabaja con ellos y se hace seguimiento de la difusión de la actividad.

Se pretende generar y promover estrategias de ocio en las que los jóvenes tengan la oportunidad de acceder a los recursos y espacios de ocio y se sientan motivados a participar en las actividades puestas en marcha, independientemente de su perfil o circunstancias sociales o personales, procurando una mayor accesibilidad a dichos recursos.

En un porcentaje similar (26,4\%) los expertos encuestados (9 participantes) han declarado que la metodología debe de ser vivencial, donde se viva y establezca el contacto con los demás y con el medio.

Se reclama que vuelvan a resurgir asociaciones de barrio dedicadas al ocio y tiempo libre que puedan movilizar a jóvenes, pero con una mayor disponibilidad de recursos económicos, y la coordinación directa de esos recursos específicos para los jóvenes.

Es relevante impulsar la creación de espacios; lugares de encuentros específicos para jóvenes donde el conocimiento mutuo y el intercambio de experiencias les fortalezca, tanto a nivel nacional como europeo. Para ello es necesario potenciar los mecanismos, canales y medios de difusión necesarios para garantizar que la información llegue a las personas destinatarias de los programas de la manera más eficaz y directa posible.

En el tema que nos ocupa, poseen un lugar destacado aquellas acciones dirigidas a la normalización de colectivos más desfavorecidos, propiciando encuentros y actividades de ocio diversas entre jóvenes de distinta procedencia. 
Por otro lado, el 23,5\% de los expertos (8 participantes) han afirmado que la metodología ha de ser procesual, puesto que las actividades no se conciben de manera aislada, sino que forman parte de un proceso individual y de grupo.

Las actividades se construyen desde un plan centrado en la persona que tiene en cuenta: la proximidad, la cercanía y el respeto; así como los intereses de los jóvenes y los problemas para poder realizar una intervención grupal multidisciplinar.

En cuanto a la participación, el 20,6\% de los profesionales encuestados (7 participantes) han declarado la importancia de una metodología en la que prime esta característica, donde las actividades se realicen entre todos, y en la que los jóvenes tengan un lugar donde expresar sus opiniones y necesidades, es decir, en la que ellos sean los protagonistas de la acción educativa.

Ante ello es importante crear espacios de encuentro y participación para la búsqueda de alternativas de ocio facilitando la integración de jóvenes con dificultades sociales, propiciando de esta manera procesos de ocio inclusivo e igualdad de oportunidades.

Finalmente, el 20,6\% de los expertos encuestados (7 participantes) han declarado que la metodología debe de ser abierta y flexible, puesto que todas las programaciones y actividades deben estar ajustadas en función del grupo y, en todo momento, abiertas a nuevas propuestas.

"Lo primero que tenemos que hacer es entender que los y las participantes son personas que pueden decidir y crear y formar su ocio, por lo que les tenemos que preguntar que desean hacer" (E27).

En este sentido es importante apoyar iniciativas de ocio organizadas y autogestionadas por los jóvenes. Con ello se otorga protagonismo a la juventud y se desarrolla con ayuda de los profesionales (apoyándoles en la cesión de espacios, equipamientos, difusión, recursos técnicos, etc.). Así mismo, se fomenta que ellos sean los protagonistas en todo momento de estas actuaciones, dejándoles plantear alternativas de ocio, tomando decisiones en grupo y valorando posteriormente sus experiencias vividas, con ánimo de que mejoren.

\section{Coordinación inter e intrainstitucional}

La siguiente red que se presenta hace referencia a la coordinación entre los profesionales que trabajan con jóvenes. Se diferencian dos tipos: intrainstitucional e interinstitucional con los aspectos más relevantes de cada una de ellas, expuestas por los expertos.

Respecto a la coordinación intrainstitucional, cabe señalar que los expertos encuestados coinciden en indicar que su principal finalidad es la coordi- 
Figura 3. Mapa conceptual análisis cualitativo. Red: Coordinación inter e intrainstitucional

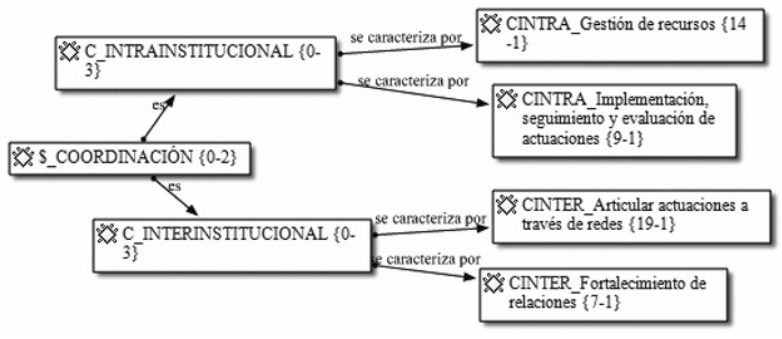

nación de actividades de ocio, mediante el desarrollo de tareas dirigidas a la población joven, en cada una de sus instituciones.

Los encuestados han indicado que las acciones más relevantes a llevar a cabo son, en primer lugar, la adecuada gestión de los recursos tanto materiales, económicos como personales (41,2\% que corresponde a 14 participantes de los 34 encuestados), de cara a optimizar las actuaciones que se llevan a cabo en base a los objetivos marcados.

Asimismo, es importante la coordinación en la implementación, seguimiento y evaluación de aquellos programas, medidas y actuaciones dirigidos a la población joven, como así lo reiteran el 26,5\% de los profesionales encuestados (9 participantes). Para ello es necesario, la creación de equipos interdisciplinares que trabajen al unísono con el objetivo de evitar duplicidades.

Con relación a la coordinación interinstitucional cabe destacar la formación de redes de trabajo para articular actuaciones, tal y como lo expresan el $55,9 \%$ de los expertos (19 participantes). El tipo de coordinación que han expuesto los profesionales se basa fundamentalmente en la difusión e intercambio de información de interés; canalización y derivación de los jóvenes a diversos servicios; participación conjunta en actividades; cofinanciación y/o subvención de proyectos y medidas concretas; así como todas aquellas iniciativas que se desarrollan conjuntamente o que colaboran en beneficio de la población joven. Las redes que se establecen pueden ser públicas y/o privadas.

Para un 20,6\% de los encuestados (7 participantes) es necesario fortalecer las relaciones con todos los actores implicados (centros educativos, federaciones, Consejerías, concejalías, asociaciones, servicios sociales, AMPAs, Diputaciones, Universidades...) para que la coordinación sea estable, periódica y efectiva hasta alcanzar el carácter de permanente. 


\section{Fuentes de Financiación}

La siguiente red guarda relación con las fuentes de financiación disponibles en los centros e instituciones que trabajan con jóvenes, para actividades relacionadas con el ocio. Reúne varios códigos destacando, sobretodo, las subvenciones públicas tanto de carácter nacional como internacionales.

Figura 4. Mapa conceptual análisis cualitativo.

\section{Red: Fuentes de Financiación}

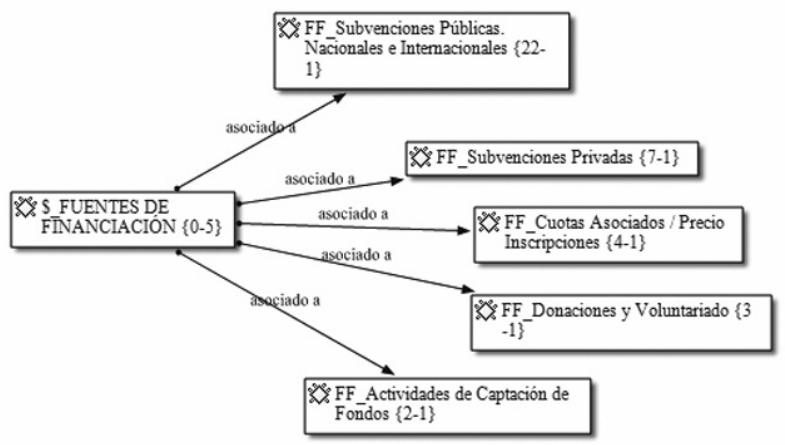

Los expertos encuestados ponen de relieve que existen, principalmente, cinco fuentes de financiación. La primera de ellas y citada por el $65 \%$ de los entrevistados (22 participantes), hace referencia a las subvenciones públicas, tanto de ámbito nacional como internacional. De ámbito nacional destacan, principalmente, las subvenciones obtenidas derivadas de los Ayuntamientos, Diputaciones y Comunidades Autónomas. Respecto al ámbito internacional, cabe destacar las ayudas obtenidas del Fondo Social Europeo.

Otra fuente de financiación hace referencia a las ayudas obtenidas por entidades privadas. El $21 \%$ de los profesionales encuestados ( 7 participantes) han declarado que reciben subvenciones de este tipo de entidades, tales como: Bancos (ej. Obra social "La Caixa"), Fundaciones (ej. Santa María) y Empresas de diversa índole.

El 12\% de los expertos (4 participantes) han destacado, como otra fuente de financiación, las cuotas de los socios y el precio de inscripción en las actividades. Esto último es considerado muy relevante, puesto que:

"Aunque sea una aportación pequeña y se utilice para el propio funcionamiento del programa, es necesario que los jóvenes puedan responsabilizarse de las actividades" (E8).

Por otra parte, un 9\% (3 participantes) han destacado las ayudas que reciben de donaciones de particulares (generalmente de personas implicadas en las ins- 
tituciones) y del voluntariado; imprescindible para llevar a cabo actividades que cuentan con pocos recursos.

Asimismo, un 6\% (2 participantes) han incidido en la importancia de las actividades de captación de fondos. Estas actividades se basan en la realización de mercadillos, prestación de servicios, fiestas de barrio y publicidad de las empresas vecinales; así como en la realización de espectáculos.

Lo más significativo que han destacado algunos de los expertos con relación a las fuentes de financiación, es que sus instituciones no disponen de financiación concreta para las actividades de ocio. Aspecto que destacan como una "dejadez" por parte de los responsables implicados y una carencia a solventar. En palabras de los encuestados...

"Nos cuesta encontrar fuentes de financiación para actividades relacionadas con el ocio juvenil" (E18).

\section{Estrategias de comunicación y difusión}

La siguiente red relativa a las estrategias de comunicación y difusión está integrada por cinco códigos. El código con mayor número de citas se refiere a la comunicación que se establece en los espacios frecuentados por los jóvenes (instituciones educativas, asociaciones...). Asimismo, otros códigos se refieren a la difusión que se realiza a través de diversos canales de comunicación.

Figura 5. Mapa conceptual análisis cualitativo. Red: Estrategias de Comunicación y Difusión

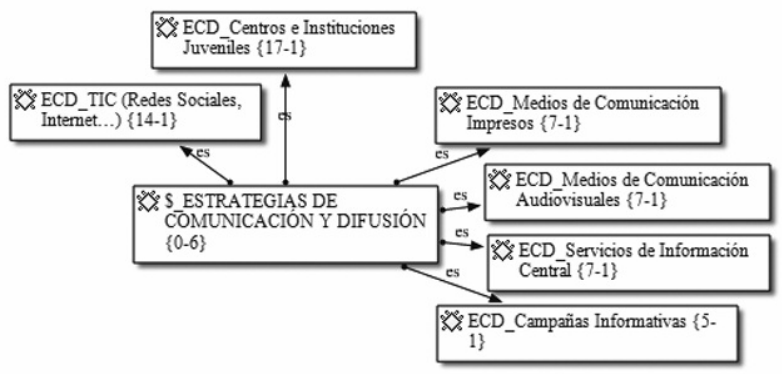

Los expertos encuestados han puesto de relieve en un 50\% (correspondiente a 17 participantes de los 34 totales), que la difusión personal (boca a boca) que se establece entre los profesionales que trabajan en las diferentes instituciones es la más efectiva para transmitir la información tanto a los jóvenes como a sus familias. Estos harán de canal multiplicador de las experiencias presentadas en el lugar en el que se desarrollan las actividades. Asimismo, algunos de los 
profesionales que trabajan con jóvenes han manifestado que se desplazan a los lugares más frecuentados por estos, con el propósito de informarles y de captar su atención e interés. Un ejemplo de ello ha sido descrito por un profesional encuestado:

"Conocer las lonjas y locales donde se reúnen a pasar tiempo. Intentar conectar con ellos y ellas. Solicitar la entrada a dichos locales, conocer los intereses y comenzar ofreciendo algo atractivo para ellos y ellas, para conseguir "enganchar", y después pasar a otro tipo de actividades" (E14).

Por su parte, un $42 \%$ de los encuestados (14 participantes) han declarado que utilizan las Tecnologías de la Información y Comunicación (Redes sociales e Internet...) como herramientas fundamentales de canalización y difusión de la información dirigida a la población joven, tales como: páginas webs, Facebook, Twitter, envío masivo de forma semanal de boletines electrónicos, envío de emails con información específica a jóvenes "susceptibles" de participar en programas concretos...Asimismo los expertos han manifestado que la comunicación debe ser actual y secuenciada, para evitar que quede obsoleta.

"La comunicación y difusión de los programas de ocio tiene que ser periódica para que la información de los mismos sea continuada en el tiempo. Puesto que ciertos recursos como los blogs, han demostrado ser una buena herramienta pero insuficiente si no existe alguna figura que canalice este espacio y le dé una continuidad" (E26).

Los participantes en el estudio han manifestado en un 21\% (7 encuestados), que utilizan para la difusión de las actividades juveniles, medios audiovisuales e impresos. Por un lado, la utilización de medios audiovisuales, tales como cuñas de radio, noticias en periódicos, tablones electrónicos y la información en televisión de carácter local. Respecto a los medios impresos destacan las publicaciones especificas (libros, revistas periódicas,...); los folletos, flyers, trípticos, buzoneo, carteles informativos, etc.

Asimismo, los profesionales destacan en un 21\% (7 encuestados) la importancia de la información que existe en los Servicios de Información Central, tales como Puntos de Información Juvenil o Servicios Sociales, ubicados en diferentes distritos de las ciudades y cuyo objetivo es la dinamización territorial y la accesibilidad a la información.

Por último, el 14\% de los expertos (5 encuestados) nombran la importancia de la implementación de campañas informativas específicas dirigidas a profesionales e interesados. Entre ellas destacan la formación de profesionales, los programas específicos de conocimiento de recursos para jóvenes, las charlas con diferentes profesionales (policías...), los talleres informativos y las campañas de voluntariado y de sensibilización. 


\section{Sistema de evaluación}

En la categoría relativa al sistema de evaluación se han ordenado las respuestas en tres subcategorías que se detallan a continuación.

Figura 6. Mapa conceptual análisis cualitativo.

\section{Red: Sistema de Evaluación}

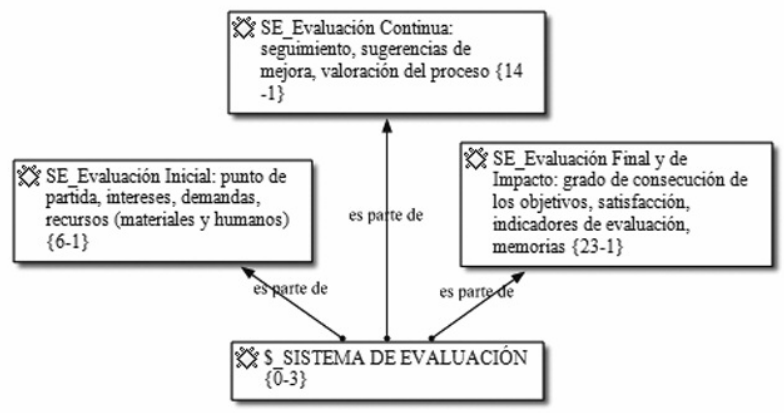

En esta red se visualizan los códigos que reúnen las citas textuales dadas por los encuestados en relación al sistema de evaluación del trabajo que realizan en materia de ocio juvenil. Como puede apreciarse el $68 \%$ de los encuestados (23 participantes de los 34 encuestados) han incidido en la importancia de la evaluación final o de impacto.

Dentro de la evaluación final los expertos han detallado una serie de indicadores de los que parten para recoger información: nivel de cumplimiento de las líneas estratégicas de las actividades/proyectos desarrollados; el nivel de idoneidad de los procedimientos organizativos; los grados de participación generales (alumnado y familias); permanencia y fidelidad de usuarios; reflexión escrita de las experiencias vividas por parte del alumnado, a través de redes sociales; elaboración de memorias anuales finales que evalúan globalmente las actividades realizadas; comprobación del grado de satisfacción de los participantes, así como la implementación de cuestionarios a colectivos y usuarios que participan o reuniones de evaluación con las familias de los participantes. Se recogen algunas citas textuales que fidelizan estas afirmaciones:

"El alumnado evalúa las actividades, así como todo el equipo docente para valorar si las actividades realizadas cumplen con los objetivos que nos planteamos al planificarlas, así como fomentan capacidades básicas del alumnado" (E13).

"Una vez finalizado el proyecto, los/as jóvenes disponen de un espacio para la reflexión de las experiencias vividas, a través de la utilización de redes sociales. Será una buena herramienta para evaluar el nivel de convivencia alcanzado entre los participantes" (E15). 
Es relevante la afirmación declarada por uno de los expertos que incide en la importancia de transferir buenas prácticas de las actividades de ocio implementadas:

"Posible transferibilidad de iniciativas que se estén realizando en otras comunidades con impacto positivo" (E4).

Asimismo el $41 \%$ de los encuestados (14 participantes) han incidido en la evaluación continua o procesual. Dependiendo de las instituciones se elaboran diferentes tipos de evaluación, tanto cuantitativa como cualitativa. La principal finalidad de esta evaluación es el "seguimiento" que se efectúa en el desarrollo de programas, en muchos de los casos mediante la implementación de indicadores de evaluación, elaborados por los profesionales que trabajan en los centros (internos), o mediante indicadores estandarizados (externos). Un ejemplo de ello es el siguiente:

"Se lleva a cabo una evaluación de cada una de las actividades, fundamentalmente a través de los/as monitores/as que ejecutan los talleres y actividades y de los profesionales responsables de cada Centro" (E21).

Finalmente, el 18\% de los expertos (6 participantes) inciden en la importancia de la evaluación inicial. Esta constituye el punto de partida para la implementación de nuevas actividades y para tener en cuenta los intereses de los jóvenes, así como sus demandas:

"Partimos de un diagnóstico participativo de necesidades". "Conociendo sus necesidades reales, comprobar si se responde a ellas, cuantas personas participan, cuantas personas se organizan" (E4).

En este sentido, es relevante gestionar los recursos tanto materiales como humanos necesarios y/o existentes para llevar a la práctica las actividades.

\section{CONCLUSIONES}

Tras el análisis y discusión de resultados, podemos concluir que el desarrollo de una buena práctica profesional desde la perspectiva del equipo de expertos y profesionales encuestados, es aquella que ha de cumplir unos mínimos requerimientos que la dote de calidad. Desde las respuestas dadas por estos 34 expertos, se han configurado unos indicadores de referencia que se pueden tomar como punto de partida para su validación posterior desde las prácticas profesionales en este campo.

- En cuanto a los objetivos de intervención, podremos considerar una práctica de calidad si: 
- La institución implementa los programas de ocio que planifica creando inquietudes saludables entre el colectivo juvenil.

- Informa y difunde las actuaciones dirigidas a jóvenes, especialmente las de su contexto cercano.

- Parte de las necesidades y demandas juveniles y desde su participación activa para la innovación en el diseño y desarrollo de las acciones.

- Orienta y motiva a la juventud mediante acciones de apoyo socioeducativo.

- Existe coordinación entre profesionales en materia de ocio juvenil para el desarrollo de acciones conjuntas y trabajo en equipo.

- Previene conductas de riesgo.

Es prioritario partir de objetivos que apuesten por intervenciones contextualizadas, que contemplen las necesidades personales del colectivo juvenil excluido, y que, desde esta situación, se diseñen planes orientados al fomento de una cohesión social plena, en orden a propiciar la inclusión social de la población joven como Paz y Unás (2010) ratifican.

- En el campo metodológico, la muestra encuestada considera que las prácticas profesionales de calidad han de reunir los siguientes requisitos:

- Metodología comunicativa.

- Lúdica y atractiva para el colectivo juvenil al que va dirigido.

- Vinculada a la realidad donde cada joven ha de ser protagonista de su proceso.

- Vivencial, conectada con el contexto de referencia.

- Procesual, desde un plan centrado en la persona y su contexto.

- Participativa; donde el joven se implique en la acción.

- Abierta y flexible.

En este sentido, estamos de acuerdo con Rodríguez (2010:43) cuando afirma que "...si unimos participación del usuario y responsabilidad del profesional, se traduce en que el plan de trabajo es necesario consensuarlo con él y ser consciente de que son procesos muy complejos y paulatinos. Ya que provocar cambios en hábitos, conductas o situaciones establecidas y perdurables no es sencillo, sino una acción muy compleja". Solo contemplando el entrelazado entre el contexto, la historia personal de cada joven y sus características, se puede realizar una intervención social de calidad.

- La coordinación inter e intrainstitucional de calidad, según estos profesionales, se matiza con los siguientes indicadores:

- Adecuada gestión de recursos para la optimización de las actuaciones. 
- Creación de equipos interdisciplinares para el diseño, implementación, seguimiento y evaluación de las actuaciones.

- Formación de redes de trabajo.

- Establecimiento y fortalecimiento de las relaciones entre todos los actores implicados en la acción.

- A nivel de financiación, según la muestra encuestada, definir una práctica profesional de calidad es partir de:

- Subvenciones públicas (tanto nacionales como internacionales).

- Financiación privada.

- Cuotas de socios.

- Precios de inscripción en las actividades.

- Donaciones particulares.

- Por otro lado, para definir una buena práctica profesional en materia de comunicación y difusión, según estos expertos, es fundamental partir de indicadores como:

- Desarrollo de un protocolo de difusión personal "boca a boca".

- Uso de las TIC.

- Uso de medios audiovisuales.

- Utilización de material impreso.

- Actualización de los tablones de los servicios de información físicos existentes.

- Diseño y puesta en marcha de campañas informativas específicas.

Trabajar la coordinación, optimizar los recursos tanto financieros como humanos, equipamientos, infraestructuras y materiales, así como de comunicación y difusión es fundamental porque posibilitará el conocimiento del contexto próximo y los factores que pueden potenciar o limitar las acciones cara a prevenir y proponer alternativas de respuesta (Observatorio del Tercer Sector de Bizkaia, 2010; Plataforma de ONG de Acción Social, 2012).

- Por último, para definir las buenas prácticas profesionales en el campo del ocio juvenil no podemos olvidar el sistema de evaluación que según los encuestados ha de partir de:

- Una evaluación diagnóstica que constituya el punto de partida en el diseño de la intervención y así partir de las demandas manifestadas por el colectivo juvenil.

- Una evaluación continua y procesual tanto cuantitativa como cualitativa que favorezca el seguimiento de las acciones.

- Una evaluación final y de impacto con indicadores específicos que midan el grado de cumplimiento de las actuaciones. 
"Los movimientos de calidad, con sus diversos modelos más o menos elaborados e implantados, reconocen en la evaluación la principal estrategia o herramienta al servicio de uno de sus principios fundamentales: el de la mejora continua" (Pérez Juste, 2000: 253).

A modo de resumen, visto desde la práctica profesional y como se desprende de este sistema de indicadores primario que emana de este estudio exploratorio, deja en evidencia un gran reto. Dicho reto pasa por crear estructuras de trabajo interprofesional que favorezcan el surgimiento de acciones contextualizadas, vinculadas a las necesidades juveniles y que emanen de la implicación de todos los actores implicados, especialmente desde la participación activa del colectivo juvenil.

En este proceso de acción, el diseño de redes de comunicación, formación, difusión y comunicación es clave a sí como la dotación de recursos que faciliten el desarrollo de lo diseñado.

A partir de aquí, el sistema de seguimiento y evaluación que se perfile será clave para ir mejorando el proceso desde la práctica; premisa que ha de guiar la práctica profesional en el ámbito del ocio.

Los profesionales encuestados, por tanto, invitan a que se tome conciencia de la filosofía de trabajo que ha de primar en las instituciones sociales que dirijen su práctica a jóvenes y ocio para, desde ello, poder crear estructuras de acción coordinadas, afianzar la red de profesionales y dotar de recursos que aboguen y primen éstas acciones cara a cumplir con un objetivo común, mejorar la calidad de vida del colectivo juvenil y favorecer su desarrollo integral y social.

\section{REFERENCIAS BIBLIOGRÁFICAS}

Birkeland, M.S., Torsheim, T., \& Wold, B. (2009). A longitudinal study of the relationship between leisure-time physical activity and depressed mood among adolescents. Psychology of Sport and Exercise, 10, 25-34.

Bisquerra, R. (2009). Psicopedagogía de las emociones. Madrid: Síntesis.

Caride, J. (2009). Ocio y Cidadania: acerca del tiempo como construción social y Educativa. En M. Cuenca y E. Aguilar (Eds.), El tiempo del Ocio: transformaciones y riesgos en la sociedad apressurada (pp. 153-176). Bilbao: Universidad de Deusto/ Instituto de Estudios del Ocio.

Cuenca, M. (2004). Pedagogía del Ocio: Modelos y Propuestas. Bilbao: Universidad de Deusto.

Cuenca, M. (2009). Perspectivas actuales de la pedagogía del ocio y el tiempo livre. En J. Otero (Ed.), La pedagogía del ocio: nuevos desafios (pp. 9-23). Lugo:Axac.

Delgado, P., Pose Porto, H. y De Valenzuela Bandín, A. L. (2015). El ocio cotidiano de los estudiantes de Educación secundaria en España. Pedagogía Social. Revista Interuniversitaria, 25, 25-49. 
Fantova, F. (2007). Repensando la intervención social. Documentación social, 147, 183198.

Fernández-García, A., Poza-Vilches, M.F. y Fiorucci, M. (2015). Análisis Metateórico sobre el Ocio de la juventud con problemas sociales. Pedagogía Social. Revista Interuniversitaria, 25, 119-141.

Giménez, J.A., Cortés, M.T. y Espejo, B (2010). Consumo de alcohol juvenil: una visión desde diferentes colectivos. Salud y Drogas, 10, 13-34.

Kovacheva, S. (2007). Constelaciones de desventaja y dilemas políticos en las transiciones de los jóvenes de la escuela al trabajo en Bulgaria. Revista de Estudios de Juventud, 77, 49-66.

López Noguero, F. (2002). El análisis de contenido como método de investigación. Revista de Educacion, 4, 167-179.

Marí, R. (2007). Propuesta de un modelo de diagnóstico en educación. Bordón, 59(4), 611-626

Observatorio del Tercer Sector de Bizkaia (2010). Guía para la gestión de proyectos sociales Recuperado de .http://www.3sbizkaia.org//Editor/Archivos/Docs/Estudios _3s\%20_def_Mayo_2014.pdf

Paz Rueda, A.L. y Unás Camelo, V. (2010). Fisuras en los discursos de la intervención social contemporánea. Revista CS, 1, 217-237.

Plataforma de ONG de Acción Social (2012). II Plan Estratégico del Tercer Sector de Acción Social 2013-2016. Madrid: Ministerio de Sanidad, Servicios Sociales e Igualdad y Plataforma de ONG de Acción Social.

Pérez-de-Guzmán, V. y Trujillo Herrera, J.F. (2011). Educar e investigar de manera participativa: la implicación asociativa juvenil en las actividades de ocio y tiempo libre. Tiempo de Educar, 12(24), 235-256.

Pérez Juste (2000). Evaluación de programas educativos. Revista de Investigación Educaciva, 18(2), 251-261.

Pérez-Serrano, G., Poza-Vilches, F. y Fernández-García, A. (2016). Criterios para una intervencion de calidad con jóvenes en dificultad social. Revista Española de Pedagogía, 263, 51-69.

Poza-Vilches, M.F. (2008). Validación empírica de un modelo de investigación-acción participativa para la implantación de agendas 21 locales en la gestión ambiental municipal. Granada: Copicentro.

Rodríguez Valladolid, N. (2010). De la relación de ayuda en la intervención social. Documentos de Trabajo Social, 48, 31-54

Stebbins, R.A. (2012). The Idea of Leisure. First Principles. New Jersey: Transaction Publishers.

Vidarte Claros, J.A. y Vélez Alvarez, C. (2012). Caracterización de la realidad juvenil de Caldas (Colombia): Área de Deporte, Recreación y Tiempo Libre. Apunts. Educación Física y Deportes, 110, 78-88. 
MARÍA DE FÁTIMA POZA-VILCHES (fatimapoza@ugr.es // ORCID: 00000001-6186-9306)

Profesora Ayudante Doctor en el Departamento MIDE (Facultad Ciencias Educación) de la Universidad de Granada. Doctora en Educación por la Universidad de Granada. Máster en Educación Social y Animación Sociocultural por la UPO.Licenciada en Pedagogía. Funcionaria (Técnico de Juventud) en el Ayuntamiento de Granada desde el año 2003-2017. Investigadora colaboradora en varios proyectos de investigación y con publicación en diferentes artículos y capítulos de libros vinculados al ámbito de la evaluación y la intervención socioambiental.

ANA FERNÁNDEZ-GARCÍA (anafernandez@edu.uned.es // ORCID: 00000002-7600-8768)

Doctora en Educación por la UNED, con premio extraordinario. Diplomada en Educación Social por la UNED, Premio Extraordinario Fin de Carrera. Licenciada en Pedagogía por la UNED. Máster en Innovación e Investigación en Educación por la UNED. Premio Extraordinario Fin de Máster en la categoría de Ciencias Sociales y Jurídicas. Como miembro de diversos proyectos de investigación, ha publicado artículos científicos en distintas Revistas, sobre la juventud en riesgo social.

JOAO PAULO DELGADO FERREIRA (pdelgado@ese.ipp.pt // ORCID: 0000001-6977-8214)

Licenciado en Derecho, maestro en Administración de la Educación y Doctor en Ciencias de la Educación por la Universidad de Santiago de Compostela, con agregación en Ciencias de la Educación en la Universidade de Trás-osMontes e Alto Douro (UTAD), es profesor adjunto en la Escola Superior de Educação do Instituto Politécnico do Porto. Es miembro integrado en el Centro de Investigação em Estudos da Criança (CIEC), da Universidade do Minho y miembro da Comissão Científica del Centro de Investigação e Inovação em Educação (INED) de la Escola Superior de Educação do Instituto Politécnico do Porto (ESEP).

Recibido: 06/04/2017

Aceptado: 16/09/2017 


\title{
DESARROLLO PERSONAL MEDIANTE LA ACTIVIDAD FÍSICA. PERCEPCIÓN DE LOS JÓVENES ESPAÑOLES SEGÚN ÁREAS GEOGRÁFICAS PERSONAL DEVELOPMENT THROUGH PHYSICAL ACTIVITY. SPANISH YOUTH'S PERCEPTION AS A FUNCTION OF GEOGRAPHICAL AREAS
}

\author{
Magdalena Sáenz de Jubera Ocón \\ Universidad de La Rioja, España \\ m-magdalena.saenz-de-jubera@unirioja.es \\ Eva Sanz Arazuri \\ Universidad de La Rioja, España \\ eva.sanz@unirioja.es \\ Emauele Isidori \\ Universidad Foro Itálico de Roma, Italia \\ labopedagogia@gmail.com
}

\begin{abstract}
Cómo citar / Citation
Sáenz de Jubera Ocón, Magdalena; Sanz Arazuri, Eva y Isidori, Emauele (2017) "Desarrollo personal mediante la actividad física. Percepción de los jóvenes españoles según áreas geográficas". OBETS. Revista de Ciencias Sociales, 12(Extra 1): pp-pp. 229-246. doi:10.14198/OBETS2017.12.1.19

\section{Resumen}

Constatado el valor educativo de la actividad física de ocio para el desarrollo personal y social de los jóvenes, este estudio pretende analizar dicha práctica y la percepción de éstos sobre el desarrollo humano atendiendo a la zona geográfica donde estudian. Se adopta un enfoque metológico de carácter cuantitativo, desarrollado mediante la aplicación de un cuestionario a una muestra de 1764 estudiantes. Se evidencia la existencia de discrepancias con las aportaciones de estudios similares en la identificación de factores prioritarios para la práctica de actividad física, y que la zona geográfica es un factor diferenciador en las atribuciones que estos jóvenes le conceden.
\end{abstract}


Palabras clave: actividad física; ocio; jóvenes; desarrollo humano; zonas geográficas.

\begin{abstract}
Having confirmed the educational value of physical leisure activity for youth's personal and social development, this study aims to analyze physical activity and students' perception of it for human development as a function of the geographical area where they study. A methodological and quantitative approach was adopted, applying a questionnaire to a sample of 1764 students. Discrepancies were found with the contributions of similar studies in the identification of priority factors for the practice of physical activity, observing that the geographical area is a differentiating factor in the attributions granted by these youngsters.
\end{abstract}

Keywords: physical activity; leisure; youth; human development; geographical areas.

\title{
Extended Abstract
}

The educational value of physical leisure activity for youth's personal and social development -which favors the physical, cognitive, emotional, behavioral, and collective aspects of human development-is confirmed. Although some studies of youth who endorse the practice of physical leisure activity from the viewpoint of human development relate physical activity to demographic variables (i.e., gender) and consider them as differentiating factors of such activity, there are few scientific studies that link physical leisure activity to geographical areas. In the same vein, some works analyze the level of physical activity by autonomous communities. Thus, the study carried out by the Consejo Superior de Deportes [CSD; Higher Sports Council) in 2011 on the sport habits of the school population in Spain or investigations like that of Ramos, Jiménez, Rivera, and Moreno (2016) do not detect significant differences between Spanish youth's practice by territories, although the CSD indicates that the autonomous communities with a higher level of physical activity present a lower rate of childhood obesity, and Ramos et al. discovered an association between physical activity and family acquisition level in the different communities.

The goal of this study is to determine whether Spanish youth's physical leisure activity and their perception of human development vary as a function of the geographical area where they are studying post-compulsory secondary education. Aspects deriving from physical practice are analyzed, for instance, physical development, application of cognitive and creative levels, enjoyment, and fun and, lastly, social relations as an outcome of physical activity. A methodological and quantitative approach was used, carried out by applying an ad hoc questionnaire to a sample of 1764 Spanish students, of whom 50.2\% were females and $49.8 \%$ were males; $13.2 \%$ were over 19 years of age, $68 \%$ were 17 or 18 years old, and $18.8 \%$ were under 17 . For each participant, the three most important leisure activities were identified in order of priority, among which physical activities were recorded if they were preferred by the students. Each student's degree of agreement with the above-mentioned benefits derived from participation in physical leisure activities was also recorded. 
Simple random sampling of the population was carried out, trying to maintain a proportional allocation in each of the 6 areas into which the Spanish territory is divided, namely: northeast (Catalonia, Aragon [except for Teruel] and the Balearic Islands), east (Valencian Community, Murcia, and Albacete), center (Madrid, Castilla-La Mancha [except for Albacete], Castilla-León [except for León, Palencia, and Burgos], Cáceres and Teruel), south (Andalusia, Canary Islands, Ceuta and Melilla), northwest (Galicia, Asturias, and León), and north (Cantabria, Basque Country, La Rioja, Navarre, Burgos and Palencia). Data analysis was carried out in two stages. Firstly, we identified the students for whom physical leisure activities are important; in a second step, we performed an inferential analysis through a one-factor analysis of variance (ANOVA) to determine the existence of differences in participants' perception of the contribution of practicing physical activity to their physical, psychological, creativecognitive, and social development as a function of the territorial area where they were studying. Variance homogeneity was tested in order to confirm the assumptions of normality and homoscedasticity. Lastly, contrasts were performed by means of multiple post-hoc comparisons. As the Levene statistic did not present equal variances, the Games-Howell test was used

The results show that Spanish students of the post-compulsory stage emphasize that the practice of physical activity supports their physical, psychological, emotional, and social development. The students' mean degree of agreement in each of designated territorial areas was higher than the median (Md = 3), firstly, highlighting their satisfaction, enjoyment, and fun as the greatest benefits, and secondly, the improvement of their physical fitness, and thirdly, their social relations. However, the creative-cognitive is least valued dimension. This study reveals that the youth from the central and eastern areas presented lower values in their perception of the assessed benefits of physical activity, whereas the youth from the northwestern and northern areas obtained the highest values. In principle, one would expect that if students from the eastern and central areas had a lower perception of all the factors of human development analyzed, they would practice less physical activity, and the Survey Sporting Habits of the Higher Sports Council of 2015 reported that, except for the students of Castilla-La Mancha, Leon, and Extremadura, these students from the eastern and central areas had the lowest level of practice of all the autonomous communities that were assigned to these areas in the study. The data obtained show the need to design and implement intervention programs from an early age and during adolescence in the entire Spanish territory in order to promote and educate people about the benefits of physical leisure activities, with particular emphasis in the eastern and central areas of Spain. This would lead to an enriching leisure that would promote Spanish youth's physical, cognitive, and social aspects of human development. It should be taken into account that adolescence is a key stage for the practice of physical activity, and hence for healthy leisure. The importance implementing these beneficial socio-educational actions at this stage is justified by studies that confirm that, as childhood goes by and children reach adolescence, they decrease their levels of physical activity and internalize behaviors that are maintained until adulthood. 


\section{INTRODUCCIÓN}

Queda constatado, con gran consenso entre la comunidad científica, el valor educativo de la la práctica de actividad física de ocio para el desarrollo personal y social de los jóvenes (Blasco, Capdevilla, Cruz, Pintanel y Valiente, 1996; Gutiérrez, 2004), lo que favorece al desarrollo humano en lo físico, lo cognitivo, lo emocional, lo conductual y lo colectivo (Caride, 1998; Cuenca, 2009; Quintana, 1991).

Son muchos los estudios que han indagado sobre los diversos factores y beneficios que la participación en actividades físicas de ocio reporta en los jóvenes, identificando su influencia positiva en la dimensión física del desarrollo humano, que contribuye a mejorar la forma física (González, 2004; Janssen, 2007; Physical Activity Guidelines Advisory Committee, 2008) lo que, consecuentemente, implica una buena respuesta coordinada de las estructuras y funciones corporales que intervienen en la práctica de actividad física (TorresLuque, Carpio, Lara y Zagazaz, 2014), contribuyendo a perfeccionar las habilidades técnicas y otras destrezas (Castro-Piñero et al., 2009; España-Romero et al., 2010).

Las ventajas y efectos positivos de la práctica de actividad física en el ámbito social quedan demostrados en la literatura científica, favoreciendo la mejora de las destrezas sociales, la comunicación y la calidad de las interacciones grupales (Reitman, O'Callaghan y Mitchell, 2005), además de constituirse en un elemento de integración social que favorece las relaciones humanas (Consejo Superior de Deportes, 2015).

Asimismo la práctica de actividad física se asocia a aspectos psicológicos y emocionales como el bienestar psicológico y la satisfacción personal (Sacker y Cable, 2006; Ussher, Owen, Cook y Whincup (2007); Ponce de León, Sanz y Valdemoros, 2009), el aumento del rendimiento y un mejor funcionamiento cognitivo (González y Portolés, 2014; Ramírez, Vinaccia y Suárez, 2004), así como la contribución a facilitar la expresión creativa (Cuenca, 2009).

Si bien existen estudios en los que los jóvenes avalan la práctica de actividad física de ocio desde el desarrollo humano y la relacionan con variables demográficas como el género (Arruza et al., 2008; Dieppa, Machargo, Luján y Guillén, 2008; Esnaola y Revuelta, 2009; Lovell, Ansari y Parker, 2010; Molero et al., 2010) o la edad (Arruza et al., 2008; Cecchini, Méndez y Muñiz, 2002; Dieppa et al., 2008), constituyéndolos en factores diferenciadores de este hábito, las investigaciones que lo vinculan con zonas geográficas apenas cuentan con representación a nivel científico. En esta línea se descubren trabajos que analizan el nivel de práctica de actividad física por comunidades autónomas; así, el estudio llevado a cabo por el Consejo Superior de Deportes (CSD) (2011) sobre los hábitos deportivos de la población escolar en España o inves- 
tigaciones como la de Ramos, Jiménez, Rivera y Moreno (2016) no detectan diferencias significativas entre la práctica de los jóvenes españoles por territorios, aunque el CSD (2011) apunta que las comunidades autónomas con un mayor nivel de actividad física son las que presentan un menor índice de obesidad infantil, y Ramos et al. (2016) descubrieron asociación entre el ejercicio de actividad física y el nivel adquisitivo de las familias en las distintas comunidades.

A partir de las premisas citadas y de la escasez de estudios sobre la temática abordada que analicen las diferencias entre distintas zonas geográficas, se lleva a cabo un estudio basado en una metodología compartida, con instrumentos rigurosos, en colaboración de distintos investigadores de diferentes lugares del país, con el objetivo de identificar las dimensiones del desarrollo humano (física, psicológica, emocional y social) más favorecidas con la práctica de actividad física según la percepción de los jóvenes españoles estudiantes de educación secundaria postobligatoria. Asimismo se investiga si esta percepción sobre el desarrollo humano proporcionado por sus prácticas de actividad física de ocio varía en función de la zona geográfica donde cursan sus estudios de educación secundaria postobligatoria. Se analizan como aspectos que se derivan de la práctica, el desarrollo físico, el implemento del nivel cognitivo y creativo, el disfrute y la diversión y, por último, las relaciones sociales.

\section{METODOLOGÍA}

\section{Participantes}

La población de estudio de esta investigación estuvo constituida por los 988.889 estudiantes de Educación Secundaria Postobligatoria del estado español censados durante el curso 2013-2014ㄹ․ El tamaño muestral previsto, para un error muestral de \pm 3 y un nivel de confianza del 99\%, debía ascender a 1850 sujetos. Tras la aplicación de los instrumentos y la validación de la muestra, el tamaño definitivo ascendió a 1764 con una mortalidad del 4.64\%.

De los 1764 alumnos, $50.2 \%$ eran mujeres $(n=885)$ y el $49.8 \%$ hombres $(n=879)$. Un $13.2 \%$ eran mayores de 19 años $(n=233)$, un 68\% tenía 17 o 18 años $(n=1.199)$ y un $18.8 \%$ era menor de 17 años $(n=332)$.

Se realizó un muestreo aleatorio simple de la población, tratando de mantener una afijación proporcional en cada una de las 6 áreas en las que se dividió al territorio español, más Ceuta y Melilla. Las 6 áreas fueron las siguientes (Tabla 1):

${ }^{1}$ Datos de alumnado matriculado en enseñanzas no universitarias, proporcionados por el Ministerio de Educación, Cultura y Deporte. 
Tabla 1 . Agrupación territorial para la muestra

\begin{tabular}{l|l}
\hline $\begin{array}{l}\text { Denominación / } \\
\text { Localización }\end{array}$ & Comunidades y/o provincias \\
\hline A1-Noreste & Cataluña, Aragón (excepto Teruel) y Baleares \\
\hline A2-Levante & Comunidad Valenciana, Murcia y Albacete \\
\hline A3-Sur & Andalucía, Islas Canarias, Ceuta y Melilla \\
\hline A4-Centro & $\begin{array}{l}\text { Madrid, Castilla-La Mancha (excepto Albacete), Castilla-León } \\
\text { (excepto León, Palencia y Burgos), Cáceres y Teruel }\end{array}$ \\
\hline A5-Noroeste & Galicia, Asturias y León \\
\hline A6-Norte & Cantabria, País Vasco, La Rioja, Navarra, Burgos y Palencia \\
\hline
\end{tabular}

\section{Instrumentos}

Siete variables conforman la estructura de este estudio:

- "Las actividades físicas son importantes en mi ocio", se trata de una variable dicotómica con dos posibles categorías: sí/no. Que registra, a través de una pregunta más amplia, si las actividades físicas ocupan un lugar prioritario entre las actividades de ocio de los jóvenes españoles.

Las cinco variables siguientes, pretende medir la percepción que tiene los estudiantes de secundaria postobligatoria sobre el desarrollo físico, el bienestar psicológico, el implemento de nivel cognitivo y creativo, y el fomento de relaciones sociales que le aporta la práctica de actividad física. Esta percepción se mide a través de una escala tipo likert de 5 puntos, donde 1 significa no estar nada de acuerdo con la expresión y 5 estar muy de acuerdo con la afirmación.

- "Cuando practico actividad física estoy más en forma, controlo mejor mis movimientos, mantengo o mejoro mi condición física".

- "Cuando practico actividad física me siento más satisfecho/a, disfruto, me divierte".

- "Cuando practico actividad física soy más creativo/a, adquiero conocimientos, aprendo cosas y amplio mi mundo".

- "Cuando practico actividad física desarrollo nuevas destrezas manuales y adquiero o perfecciono habilidades técnicas".

- "Cuando practico actividad física hago cosas diferentes con más gente, me siento parte de un grupo, me ayuda a relacionarme mejor con los demás".

Finalmente, el área geográfica en que reside el alumno, es la última variable contemplada, se trata de una variable nominal conformada por 6 posibles categorías ya descritas en el apartado de población y muestra. 
Para la recogida de información de estas siete variables, se empleó un instrumento mucho más amplio y complejo que permitió registrar datos para una investigación nacional coordinada de la que este trabajo formó parte. Dicho instrumento fue validado a través de una prueba piloto realizada en 8 comunidades autónomas y valorado por 14 expertos pertenecientes a siete universidades españolas, que dieron su visto bueno para la aplicación definitiva, su fiabilidad también fue contrastada.

\section{Procedimiento}

La aplicación del cuestionario se realizó de forma aleatoria entre los estudiantes de los distintos centros educativos de cada una de las seis áreas geográficas del estado español. Previa aplicación del instrumento se solicitó permiso tanto al Director General de Educación de cada Comunidad Autónoma, como a los directores de los centros educativos, y se informó de los pormenores de la investigación. Para la aplicación de los instrumentos, dos investigadores debidamente formados acudieron personalmente a cada centro para informar al profesorado sobre el cumplimiento del cuestionario para reducir, así, la mortalidad experimental.

\section{Análisis de resultados}

Dos fases definen el análisis de los datos realizado. En primer lugar, se identifican aquellos estudiantes para los que las actividades físicas son importantes en su ocio; en un segundo paso, se llevó a cabo un análisis inferencial a través de la prueba de varianza de un factor (ANOVA de un factor), que permitió comprobar la existencia de diferencias en la percepción sobre la aportación que la práctica de actividad física ofrece al desarrollo físico, psicológico, creativo-cognitivo y social en función del área territorial en el que estudian los participantes en la muestra. Se probó la homogeneidad de las varianzas con el fin de acreditar los supuestos de normalidad y homocedasticidad a través del estadístico de Levene. Finalmente, se efectuaron contrastes mediante comparaciones múltiples post-hoc; dado que el estadístico de Levene hizo sospechar que las varianzas no eran similares se empleó la prueba Games-Howell.

El nivel de significatividad considerado en todo momento fue $\mathrm{p}<0.05$.

\section{RESULTADOS Y DISCUSIÓN}

El 48.4\% ( $n=854)$ de los estudiantes de Educación Secundaria Postobligatoria del estado español identifican al menos una actividad física entre sus tres actividades de ocio más importantes. A partir de estas líneas el estudio se centra exclusivamente en esta proporción. 
Los estudiantes valoran que la práctica de actividad física les ayuda en su desarrollo físico, psicológico, emocional y social. Se obtiene una media del grado de acuerdo de los estudiantes en cada uno de los ámbitos señalados superior a la mediana $\left(M_{e}=3\right)$, destacando, en primer lugar, como mayor beneficio la satisfacción, el disfrute y la diversión $(\bar{\chi}=3.83 \pm 1.861)$; en segunda instancia, la mejora de la forma y la condición física $(\bar{\chi}=3.35 \pm 1.859)$; y en tercer lugar, relaciones sociales $(\bar{\chi}=3.30 \pm 1.891)$ (Tabla 2). Sin embargo, la dimensión menos valorada es la creativo-cognitiva $(\bar{\chi}=2.75 \pm 1.718$ ) (Tabla 2).

Tabla 2. Estadísticos descriptivos: Percepción del estudiante de Educación Secundaria Postobligatoria sobre el desarrollo humano que aporta la actividad física

\begin{tabular}{l|c}
\hline Cuando práctico actividad física... & $\bar{\chi} \pm \mathrm{ds}$ \\
\hline $\begin{array}{l}\text {...estoy más en forma, controlo mejor mis movimientos, } \\
\text { mantengo o mejoro mi condición física }\end{array}$ & $3.35 \pm 1.859$ \\
\hline$\ldots$ me siento más satisfecho/a, disfruto, me divierte & $3.83 \pm 1.861$ \\
\hline $\begin{array}{l}\text {...soy más creativo/a, adquiero conocimientos, aprendo } \\
\text { cosas y amplio mi mundo }\end{array}$ & $2.75 \pm 1.718$ \\
\hline $\begin{array}{l}\text {...desarrollo nuevas destrezas manuales y adquiero o } \\
\text { perfecciono habilidades técnicas }\end{array}$ & $3.22 \pm 1.871$ \\
\hline $\begin{array}{l}\text {...hago cosas diferentes con más gente, me siento parte de } \\
\text { un grupo, me ayuda a relacionarme mejor con los demás }\end{array}$ & $3.30 \pm 1.891$ \\
\hline
\end{tabular}

Fuente: elaboración propia

Los resultados del ANOVA de un factor rechazan la igualdad de las varianzas poblacionales analizadas, y muestran diferencias significativas al menos entre dos áreas territoriales, en cuanto al grado de acuerdo del estudiante de Educación Secundaria Postobligatoria sobre cada uno de los ámbitos que la actividad física les permite desarrollar (Tabla 3).

Al profundizar en estas diferencias significativas, a partir de comparaciones múltiples mediante la prueba de Games-Howell, se identifica a los estudiantes del área centro $(\bar{\chi}=2.971)$ y de la zona de levante $(\bar{\chi}=2.979)$ como los que menos consideran que su actividad física les permite mejorar su forma y condición física. Así, los estudiantes de ambas áreas creen significativamente menos que los de las áreas $\operatorname{sur}(\bar{\chi}=3.666)$, noroeste $(\bar{\chi}=4.035)$ y norte $(\bar{\chi}$ $=3.801$ ) en la mejora de su forma y condición física a través de la práctica. Los valores de $p=.004$ y .022; .000 y .000; y .001 y .006 respectivamente. Por 
Tabla 3: Resumen ANOVA de un factor: Percepción del estudiante de Educación Secundaria Postobligatoria sobre el desarrollo humano que aporta la actividad física y área territorial en la que ejerce el docente

\begin{tabular}{|c|c|c|c|c|c|c|c|c|}
\hline $\begin{array}{l}\text { Cuando práctico } \\
\text { actividad física... }\end{array}$ & $\begin{array}{l}\text { Estadístico } \\
\text { de Levene }\end{array}$ & $p$ & & $\begin{array}{c}\text { Suma de } \\
\text { cuadrados }\end{array}$ & gl & $\begin{array}{c}\text { Medida } \\
\text { cuadrática }\end{array}$ & F & $p$ \\
\hline $\begin{array}{l}\text {...estoy más en } \\
\text { forma, controlo } \\
\text { mejor } \\
\text { movimientos, } \\
\text { mantengo } \\
\text { mejoro condición } \\
\text { física }\end{array}$ & 14.912 & .000 & \begin{tabular}{|} 
Intergrupos \\
Intragrupos \\
Total
\end{tabular} & $\begin{array}{l}127.202 \\
2822.885 \\
2950.087\end{array}$ & $\begin{array}{l}848 \\
853\end{array}$ & 25.440 & 7.642 & $.000 *$ \\
\hline $\begin{array}{l}\text {...me siento más } \\
\text { satisfecho/a, } \\
\text { disfruto, me } \\
\text { divierte }\end{array}$ & 39.762 & .000 & $\mid \begin{array}{c}\text { Intergrupos } \\
\text { Intragrupos } \\
\text { Total }\end{array}$ & $\begin{array}{c}176.977 \\
2779.073 \\
2956.050\end{array}$ & $\begin{array}{c}5 \\
848 \\
853\end{array}$ & $\begin{array}{l}35.395 \\
3.277\end{array}$ & 10.800 & $.000 *$ \\
\hline $\begin{array}{l}\text {...soy más } \\
\text { creativo/a, } \\
\text { adquiero } \\
\text { conocimientos, } \\
\text { aprendo cosas y } \\
\text { amplio mi mundo }\end{array}$ & 8.289 & .000 & $\begin{array}{c}\text { Intergrupos } \\
\text { Intragrupos } \\
\text { Total }\end{array}$ & $\begin{array}{c}87.136 \\
2430.849 \\
2517.985\end{array}$ & $\begin{array}{c}5 \\
848 \\
853\end{array}$ & $\begin{array}{l}17.427 \\
2.867\end{array}$ & 6.079 & $.000 *$ \\
\hline $\begin{array}{l}\text {...desarrollo } \\
\text { nuevas destrezas } \\
\text { manuales y } \\
\text { adquiero o } \\
\text { perfecciono } \\
\text { habilidades } \\
\text { técnicas }\end{array}$ & 14.391 & .000 & \begin{tabular}{|} 
Intergrupos \\
Intragrupos \\
Total
\end{tabular} & $\begin{array}{c}158.677 \\
1830.334 \\
2989.011\end{array}$ & $\begin{array}{c}5 \\
848 \\
853\end{array}$ & $\begin{array}{l}31.735 \\
3.338\end{array}$ & 9.508 & $.000 *$ \\
\hline $\begin{array}{l}\text {...hago cosas } \\
\text { diferentes con más } \\
\text { gente, me siento } \\
\text { parte de un grupo, } \\
\text { me ayuda a } \\
\text { relacionarme }\end{array}$ & 19.588 & .000 & \begin{tabular}{|c|} 
Intergrupos \\
Intragrupos \\
Total
\end{tabular} & $\begin{array}{c}147.942 \\
2905.052 \\
3052.994\end{array}$ & $\begin{array}{c}5 \\
848 \\
853\end{array}$ & $\begin{array}{l}29.588 \\
3.426\end{array}$ & 8.637 & $.000 *$ \\
\hline
\end{tabular}

${ }^{1}$ Prueba de Levene: $p<0.05$ en todos los casos luego no se asume la igualdad de las varianzas Fuente: elaboración propia 
otro lado, los estudiantes de la zona noroeste $(\bar{\chi}=4.035)$ solo se diferencian significativamente con los de la zona noreste $(\bar{\chi}=3.382)$ a favor de la última, con un valor de $p=.028$. Entre el resto de áreas no existen diferencias significativas (Tabla 4).

Tabla 4: Comparaciones múltiples mediante Games-Howell: Análisis de la Percepción del estudiante de Educación Secundaria Postobligatoria sobre la mejora de la forma y la condición física que aporta la actividad física y área territorial en la que estudia

\begin{tabular}{l|l|r|r|r}
\hline (I) Área & (J) Área & Diferencia de medias (I-J) & Error típico & $p$ \\
\hline \multirow{2}{*}{ A1- Noreste } & A2- Levante & .40219 & .227 & .488 \\
& A3- Sur & -.28452 & .212 & .762 \\
& A4- Centro & .41047 & .202 & .327 \\
& A5- Noroeste & $-.65357^{*}$ & .212 & .028 \\
& A6- Norte & -.41944 & .224 & .422 \\
\hline \multirow{2}{*}{ A2- Levante } & A3- Sur & $-.68672^{*}$ & .217 & .022 \\
& A4- Centro & .00828 & .207 & 1.000 \\
& A5- Noroeste & $-1.05576^{*}$ & .218 & .000 \\
& A6- Norte & $-.82164^{*}$ & .229 & .006 \\
\hline \multirow{2}{*}{ A3- Sur } & A4- Centro & $.69499^{*}$ & .190 & .004 \\
& A5- Noroeste & -.36905 & .202 & .451 \\
& A6- Norte & -.13492 & .213 & .989 \\
\hline \multirow{2}{*}{ A4- Centro } & A5- Noroeste & $-1.06404^{*}$ & .191 & .000 \\
& A6- Norte & $-.82991^{*}$ & .204 & .001 \\
\hline A5- Noroeste & A6- Norte & .23413 & .214 & .884 \\
\hline
\end{tabular}

$P<.005$

Sobre la satisfacción, el disfrute y la diversión que aporta la actividad física, las comparaciones múltiples mediante la prueba de Games-Howell indican que los estudiantes de Secundaria Postobligatoria del área noroeste $(\bar{\chi}=4.525)$ perciben que la práctica física les divierte y satisface en igual medida que los alumnos del noreste $(\bar{\chi}=4.034)$ y norte $(\bar{\chi}=4.565)$. Sin embargo, existen diferencias significativas entre los del noreste y norte $(p=.035)$ a favor de los últimos. Estas diferencias, a favor de los alumnos del norte, se extienden al resto de zonas: levante $(\bar{\chi}=3.382 ; p=.000)$, sur $(\bar{\chi}=4.004 ; p=.014)$ y centro $(\bar{\chi}=$ $3.370 ; p=.000)$. De igual modo, los discentes de Secundaria Postobligatoria del área levante $(\bar{\chi}=3.382)$ sienten por igual que la actividad física les divierte y satisface en igual medida que los alumnos del sur $(\bar{\chi}=4.004)$ y centro $(\bar{\chi}=$ 3.370). Sin embargo, existen diferencias significativas entre los del sur y centro 
$(p=.017)$ a favor de los estudiantes del sur; sin embargo, los sureños se divierten significativamente menos que los del noroeste $(\bar{\chi}=4.525 ; p=.024)$ y norte $(\bar{\chi}=4.565 ; p=.014)$. En cuanto a los alumnos del área centro $(\bar{\chi}=3.370)$, estos se muestran igual de satisfechos con la práctica física que sus homologos de levante $(\bar{\chi}=3.338)$, pero significativamente menos que los de las demás zonas: noreste $(\bar{\chi}=4.034 ; p=.006)$, sur $(\bar{\chi}=4.004 ; p=.001)$, noroeste $(\bar{\chi}=4.525 ; p=$ .000) y norte $(\bar{\chi}=4.565 ; p=.000)$ (Tabla 5).

Tabla 5: Comparaciones múltiples mediante Games-Howell: Análisis de la Percepción del estudiante de Educación Secundaria Postobligatoria sobre la satisfacción, el disfrute y la diversión que aporta la actividad física y área territorial en la que estudia

\begin{tabular}{l|l|r|r|r}
\hline (I) Área & (J) Área & Diferencia de medias (I-J) & Error típico & \multicolumn{1}{c}{$p$} \\
\hline A1- Noreste & A2- Levante & .65239 & .229 & .054 \\
& A3- Sur & .03050 & .204 & 1.000 \\
& A4- Centro & $.66442^{*}$ & .201 & .014 \\
& A5- Noroeste & -.49110 & .173 & .056 \\
& A6- Norte & $-.53098^{*}$ & .177 & .035 \\
\hline A2- Levante & A3- Sur & -.62189 & .224 & .066 \\
& A4- Centro & .01203 & .222 & 1.000 \\
& A5- Noroeste & $-1.14349^{*}$ & .196 & .000 \\
& A6- Norte & $-1.18337^{*}$ & .200 & .000 \\
\hline A3- Sur & A4- Centro & $.63392^{*}$ & 195 & .017 \\
& A5- Noroeste & $-.52160^{*}$ & .166 & .024 \\
& A6- Norte & $-.56148^{*}$ & .170 & .014 \\
\hline A4- Centro & A5- Noroeste & $-1.15552^{*}$ & .163 & .000 \\
& A6- Norte & $-1.19540^{*}$ & .167 & .000 \\
\hline A5- Noroeste & A6- Norte & -.03988 & .132 & 1.000 \\
\hline P<.005 & \multicolumn{3}{|c}{}
\end{tabular}

$P<.005$

Ante la mejora de creatividad que aporta la actividad física, los estudiantes del área centro $(\bar{X}=2.387)$ no consideran que su actividad física les permita desarrollar su creatividad y adquisición de conocimientos tanto como los de las áreas noreste $(\bar{\chi}=2.950)$, sur $(\bar{\chi}=2.953)$, noroeste $(\bar{\chi}=3.269)$, y norte $(\bar{\chi}=$ 3.098), con unos valores de $p=.020, .012, .011$ y .011 respectivamente; levante es el único área con el que la zona centro no registra diferencias significativas. A su vez, levante $(\bar{\chi}=2.494)$ solo muestra diferencias significativas con el área noroeste $(\bar{\chi}=3.269)$, siendo mayor el aumento de creatividad percibida por los estudiantes del noroeste cuando practican actividad física $(p=.014)$. 
Tabla 6: Comparaciones múltiples mediante Games-Howell: Análisis de la Percepción del estudiante de Educación Secundaria Postobligatoria sobre la mejora de la creatividad que aporta la actividad física y área territorial en la que estudia

\begin{tabular}{l|l|r|r|r}
\hline (I) Área & (J) Área & Diferencia de medias (I-J) & Error típico & \multicolumn{1}{c}{$p$} \\
\hline \multirow{2}{*}{ A1- Noreste } & A2- Levante & .45612 & .205 & .227 \\
& A3- Sur & -.00302 & .195 & 1.000 \\
& A4- Centro & $.56390^{*}$ & .178 & .020 \\
& A5- Noroeste & -.31867 & .233 & .749 \\
& A6- Norte & -.14807 & .233 & .988 \\
\hline \multirow{2}{*}{ 42- Levante } & A3- Sur & -.45914 & .198 & .191 \\
& A4- Centro & .10778 & .181 & .991 \\
& A5- Noroeste & $-.77479^{*}$ & .235 & .014 \\
& A6- Norte & -.60419 & .235 & .108 \\
\hline \multirow{2}{*}{ A3- Sur } & A4- Centro & $.56692^{*}$ & .171 & .012 \\
& A5- Noroeste & -.31565 & .228 & .737 \\
& A6- Norte & -.14505 & .228 & .988 \\
\hline \multirow{2}{*}{ A4- Centro } & A5- Noroeste & $-.88257^{*}$ & .213 & .011 \\
& A6- Norte & $-.71197^{*}$ & .213 & .011 \\
\hline A5- Noroeste & A6- Norte & .17060 & .261 & .987 \\
\hline
\end{tabular}

$P<.005$

Al profundizar en estas diferencias significativas a partir de comparaciones múltiples mediante la prueba de Games-Howell, se identifica a los estudiantes del área centro $(\overline{\mathcal{X}}=2.708)$ y de la zona de levante $(\overline{\mathcal{X}}=2.937)$, como los que menos consideran que su actividad física les permite desarrollar destrezas manuales y habilidades técnicas. En concreto, los estudiantes de Secundaria Postobligatoria de las áreas noreste $(\overline{\mathcal{X}}=3.427)$, sur $(\overline{\mathcal{X}}=3.456)$, noroeste $(\bar{\chi}=$ 4.021) y norte $(\bar{\chi}=3.613)$ perciben significativamente mayor desarrollo de sus destrezas manuales y habilidades técnicas que los alumnos de la zona centro $(\bar{\chi}=2.708)$ con unos valores de $p=.006, .001, .000$ y .000 respectivamente; de igual modo, los jóvenes del noroeste $(\bar{\chi}=4.021)$ y del norte $(\bar{\chi}=3.613)$ también sienten en mayor medida que sus homólogos de levante que la práctica física les desarrolla destrezas manuales y habilidades técnicas, con unos valores de $p=.000$ y .041 respectivamente. Entre el resto de áreas no existen diferencias significativas (Tabla 7). 
Tabla 7: Comparaciones múltiples mediante Games-Howell: Análisis de la Percepción del estudiante de Educación Secundaria Postobligatoria sobre el desarrollo de destrezas manuales y habilidades técnicas que aporta la actividad física y área territorial en la que estudia

\begin{tabular}{l|l|r|r|r}
\hline (I) Área & (J) Área & Diferencia de medias (I-J) & Error típico & \multicolumn{1}{c}{$p$} \\
\hline A1- Noreste & A2- Levante & .48999 & .233 & .290 \\
& A3- Sur & -.02890 & .213 & 1.000 \\
& A4- Centro & $.71859^{*}$ & .201 & .006 \\
& A5- Noroeste & -.59438 & .215 & .068 \\
& A6- Norte & -.18581 & .220 & .959 \\
\hline \multirow{2}{*}{ A2- Levante } & A3- Sur & -.51889 & .223 & .187 \\
& A4- Centro & .22860 & .211 & .889 \\
& A5- Noroeste & $-1.08437^{*}$ & .224 & .000 \\
& A6- Norte & $-.67580^{*}$ & .229 & .041 \\
\hline \multirow{2}{*}{ A3- Sur } & A4- Centro & $.74749^{*}$ & .190 & .001 \\
& A5- Noroeste & -.56548 & .204 & .066 \\
& A6- Norte & -.15691 & .209 & .976 \\
\hline \multirow{2}{*}{ A4- Centro } & A5- Noroeste & $-1.31297^{*}$ & .191 & .000 \\
& A6- Norte & $-.90440^{*}$ & .211 & .000 \\
\hline \multirow{2}{*}{ A5- Noroeste } & A6- Norte & .40857 & .211 & .385 \\
\hline
\end{tabular}

$P<.005$

Finalmente, de nuevo los estudiantes del área centro $(\bar{\chi}=2.868)$ y de la zona de levante $(\bar{\chi}=2.870)$, son los que menos consideran que su actividad física favorece sus relaciones sociales. Concretamente, los estudiantes de Secundaria Postobligatoria de las áreas noreste $(\bar{\chi}=3.507)$, sur $(\bar{\chi}=3.589)$, noroeste $(\bar{\chi}=$ 3.974), y norte $(\bar{\chi}=3.773)$, significativamente perciben que sus relaciones sociales aumentan en mayor medida que los alumnos de la zona centro $(\bar{\chi}=$ 2.868) con unos valores de $p=.025, .003, .000$ y .000 respectivamente; de igual modo, los jóvenes del sur $(\bar{\chi}=3.589)$ noroeste $(\bar{\chi}=3.974)$ y del norte $(\overline{\mathcal{X}}=3.773)$ también sienten en mayor medida que sus homologos de levante que la práctica física fomenta sus relaciones sociales, con unos valores de $p=.016, .000$ y .001 respectivamente. Entre el resto de áreas no existen diferencias significativas (tabla 8). 
Tabla 8: Comparaciones múltiples mediante Games-Howell: Análisis de la Percepción del estudiante de Educación Secundaria Postobligatoria sobre el fomento de las relaciones sociales que aporta la actividad física y área territorial en la que estudia

\begin{tabular}{l|l|r|r|r}
\hline (I) Área & (J) Área & Diferencia de medias (I-J) & Error típico & \multicolumn{1}{c}{$p$} \\
\hline \multirow{2}{*}{ A1- Noreste } & A2- Levante & .63617 & .232 & .071 \\
& A3- Sur & -.8244 & .212 & .999 \\
& A4- Centro & $.63826^{*}$ & .205 & .025 \\
& A5- Noroeste & -.46705 & .211 & .237 \\
& A6- Norte & -.26681 & .219 & .828 \\
\hline \multirow{2}{*}{ 42- Levante } & A3- Sur & $-.71861^{*}$ & .221 & .016 \\
& A4- Centro & .00209 & .215 & 1.000 \\
& A5- Noroeste & $-1.10322^{*}$ & .220 & .000 \\
& A6- Norte & $-.90298^{*}$ & .227 & .001 \\
\hline \multirow{2}{*}{ A3- Sur } & A4- Centro & $.72070^{*}$ & .193 & .003 \\
& A5- Noroeste & -.38461 & .199 & .386 \\
& A6- Norte & -.18437 & .207 & .949 \\
\hline \multirow{2}{*}{ A4- Centro } & A5- Noroeste & $-1.10531^{*}$ & .192 & .000 \\
& A6- Norte & $-.90507^{*}$ & .200 & .000 \\
\hline A5- Noroeste & A6- Norte & .20024 & .206 & .927 \\
\hline
\end{tabular}

$P<.005$

\section{CONCLUSIONES}

Tal y como reflejan los resultados obtenidos, los estudiantes españoles de la etapa postobligatoria destacan como ámbitos que la actividad física les permite desarrollar la satisfacción, el disfrute y la diversión, seguido de una mejora de la forma y la condición física, y del incremento de sus relaciones sociales. Sin embargo, entre los beneficios de estas prácticas conceden menor importancia al crecimiento creativo-cognitivo. Estos resultados a nivel estatal muestran algunas discrepancias con las evidencias aportadas por la última encuesta de hábitos saludables (Consejo superior de Deportes, 2015) que identifica como factores positivos prioritarios para la práctica de actividad física, el desarrollo físico $(29,9 \%)$, seguido por orden de importancia de la diversión o entretenimiento $(23 \%)$, si bien con mucha menor frecuencia se encuentra el fomento de la relación social derivada de la práctica $(2,6 \%)$. Asimismo, los resultados de este trabajo entran en contradicción con las aportaciones de Ramírez et al. (2004), que evidenció en los practicantes una mejora del rendimiento y de la cognición. 
Si atendemos a la zona geográfica de donde proceden los estudiantes, se comprueba que es un factor diferenciador en las atribuciones de los estudiantes de Educación Secundaria Postobligatoria a la práctica de actividad física de ocio, siendo los estudiantes de la zona Centro y de Levante los que menos consideran que la actividad física les permita mejorar su forma y condición física, por debajo de las creencias del alumnado del sur, noroeste y norte.

Son los discentes del área Noroeste, Norte y Noreste, los que perciben que la práctica de actividad física contribuye más a su desarrollo personal en lo referente a satisfacción, disfrute y diversión, existiendo diferencias a favor de los alumnos del norte respecto al noreste y a las otras zonas analizadas.

Respecto al desarrollo de la creatividad y la adquisición de conocimientos son los estudiantes de la zona centro y los de Levante los que señalan que la actividad física no favorece la mejora de estas capacidades, siendo más valoradas por el alumnado de las áreas noreste, sur, noroeste y norte. Asimismo, son de nuevo los estudiantes de las áreas centro y levante los que perciben menor aportación de la actividad física al desarrollo de sus destrezas manuales y habilidades técnicas respecto a los alumnos del noreste, sur, noroeste y norte. Si bien los jóvenes de estas dos últimas zonas son los que más sienten que la práctica física contribuye a la mejora de estas habilidades.

Los estudiantes del área centro y Levante son los menos consideran de nuevo que su actividad física favorece las relaciones sociales. Los de las áreas Noreste, sur, noroeste y Norte perciben respecto a los del Centro y Levante que sus relaciones aumentan con la práctica física.

A través de este estudio se evidencia que los jóvenes de la zona Centro y Levante muestran los valores más bajos en la percepción del conjunto de actitudes valoradas que se derivan de la práctica, siendo los de la zona Noroeste y Norte los que significan valores más altos. En principio, cabría esperar que si existe menor percepción en todos los factores del desarrollo humano analizados en las áreas de Levante y del Centro, se practicará menos actividad física, si bien la Encuesta de Hábitos Deportivos (Consejo Superior de Deportes, 2015) refleja que de todas las comunidades autónomas que se han adscrito en el estudio a estas zonas, tienen un nivel de práctica más bajo salvo Castilla La Mancha, León y Extremadura.

Tras los datos obtenidos, se sugiere la necesidad de diseñar e implementar programas de intervención en todo el territorio español, desde edades tempranas y en la adolescencia, para promocionar y concienciar a los individuos sobre los beneficios que reporta la participación en actividades físicas de ocio, incidiendo en su puesta en marcha en las zonas de Levante y Centro de España, que lleven a vivenciar un ocio enriquecedor que favorezca el desarrollo huma- 
no en lo físico, lo cognitivo y lo social de los jóvenes españoles (Caride, 1998; Cuenca, 2009; Quintana, 1991), siendo la adolescencia una etapa clave para la práctica de actividad física, y por ende para el ejercicio de un ocio saludable. La importancia de articular acciones socioeducativas en esta etapa que redunden en las bondades de la misma se justifica en la existencia de estudios como el de Ramos et al. (2016) que constatan que conforme va transcurriendo la infancia y se va alcanzando la adolescencia, los chicos y chicas van disminuyendo el nivel de práctica de actividad física e interiorizan conductas que pueden permanecer hasta la adultez (Heaven, 1996).

\section{REFERENCIAS BIBLIOGRÁFICAS}

Arruza, J. A., Arribas, S., Gil De Montes, L., Irazusta, S., Romero, S. y Cecchini, J.A. (2008). Repercusiones de la duración de la actividad físico-deportiva sobre el bienestar psicológico. Revista Internacional de Medicina y Ciencias de la Actividad Física y el Deporte, 8(30), 171-183. Disponible en http://goo.gl/X10q62

Blasco, T., Capdevilla, L., Cruz, J., Pintanel, M. y Valiente L. (1996). Evolución de los patrones de actividad física en estudiantes universitarios. Revista de Psicología del Deporte, 9-10, 51-63.

Caride, J.A. (1998). Educación del ocio y del tiempo libre. En M. Beas, y J. García (Coords.), Atención a los espacios y tiempos escolares (pp. 17-31). Granada: Grupo Editorial Universitario.

Castillo, I. y Molina-García, J. (2009). Adiposidad corporal y bienestar psicológico: efectos de la actividad física en universitarios de Valencia, España. Revista Panamericana de Salud Publica, 26(4).

Castro-Piñero, J., Gonzalez-Montesinos, J. L., Mora, J., Keating, X. D., Girela-Rejon, M. J., Sjostrom, M. y Ruiz, J. R. (2009). Percentile values for muscular strength field tests in children aged 6 to 17 years: influence of weight status. J Strength Cond Res, 23 (8), 2295-2310.

Cecchini, J. A., Méndez, A. y Muñiz, J. (2002). Motivos de práctica deportiva en escolares españoles. Psicothema. 14, 523-531.

Consejo Superior de Deportes (2011). Hábitos deportivos de la población escolar en España. Recuperado de www.csd.gob.es/csd/estaticos/de-escolar/encuesta-dehabitosdeportivos-poblacion-escolar-en-espana.pdf

Consejo Superior de Deportes (2015). Hábitos deportivos de la población escolar en España. Recuperado de http: http://www.mecd.gob.es/servicios-al-ciudadanomecd/dms/mecd/servicios-al-ciudadano-mecd/estadisticas/deporte/ehd/Encuesta_ de_Habitos_Deportivos_2015.pdf

Cuenca, M. (2009). Ocio humanista. Dimensiones y manifestaciones actuales del ocio. Bilbao: Universidad de Deusto.

Dieppa, M., Machargo, J., Luján, I. y Guillén, F. (2008). Autoconcepto general y físico en jóvenes españoles y brasileños que practican actividad física vs. no practicantes. Revista de Psicología del Deporte, 17(2), 221-239. 
Esnaola, I. y Revuelta, L. (2009). Relaciones entre la actividad física, autoconcepto físico, expectativas, valor percibido y dificultad percibida. Acción psicológica, 6(2), 31-43.

España-Romero, V., Artero, E. G., Jiménez-Pavón, D., Cuenca-García, M., Ortega, F. B., Castro-Piñero, J., Sjostrom, M., Castillo-Garzón, M. J. y Ruiz, J. R. (2010). Assessing health-related fitness tests in the school setting: reliability, feasibility and safety; the ALPHA Study. Int J Sports Med, 31 (7), 490-497.

González, J. (2004). La actividad física orientada a la promoción de la salud. Revista de Investigación Educativa, 7, 73-96.

González, J. y Portolés, A. (2014). Actividad física extraescolar: relaciones con la motivación educativa, rendimiento académico y conductas asociadas a la salud. Revista Iberoamericana de Psicología del Ejercicio y el Deporte, 9(1), 51-65.

Gutierrez, M. (2004). El valor del deporte en la educación integral del ser humano. Revista de Educación, 335, 105-126.

Heaven, P. C. L. (1996). Adolescent health: The role of individual differences. Londres: Routledge.

Janssen, I. (2007). Physical activity guidelines for children and youth. Applied Physiology Nutrition and Metabolism, 32, 109-121.

Lovell, G.P., Ansari, W.E. y Parker, J.K. (2010). Perceived Exercise Benefits and Barriers of Non-Exercising Female University Students in the United Kingdom. International Journal of Environmental Research and Public Health, 7, 784-798.

Molero, D., Ortega, F., Valiente, I. y Zagalaz, M.L. (2010). Estudio comparativo del autoconcepto físico en adolescentes en función del género y del nivel de actividad físico-deportiva. Retos. Nuevas tendencias en Educación Física, Deporte y Recreación, 17, 38-41.

Physical Activity Guidelines Advisory Committee (PAGAC). (2008). PhysicalActivity Guidelines Advisory Committee Report, 2008. Washington, DC, US Department of Health and Human Services.

Ponce de León, A., Sanz, E., Valdemoros, M.A., y Ramos, R. (2009). Los valores personales en el ocio físico-deportivo. Un estudio con jóvenes, padres y profesores. Bordón, 61, 1, pp. 29-41.

Quintana, J.M. (1991). El tiempo libre como ámbito humano y cultural. En J.M. Quintana (Ed.), Iniciativas sociales en educación informal (pp. 402-415). Madrid: Rialp.

Ramírez, W., Vinaccia, S. y Suárez, G. (2004). El impacto de la actividad física y el deporte sobre la salud, la cognición, la socialización y el rendimiento académico: una revisión teórica. Revista de Estudios Sociales, 18, 67-75.

Ramos, P., Jiménez-Iglesias, A., Rivera, F. y Moreno, C. (2016). Evolución de la práctica de la actividad física en los adolescentes españoles / Physical Activity Trends in Spanish Adolescents. Revista Internacional de Medicina y Ciencias de la Actividad Física y el Deporte, 16 (62), 335-353. http://dx.doi.org/10.15366/rimcafd2016.62.010

Reitman, D., O'Callaghan, P. M. y Mitchel, P. (2005). Parent as coach: enhancing sports participation and social behaviour for ADHD-diagnosed children. Child and Family Behavior Therapy, 27, 2, 57-68. http://dx.doi.org/10.1300/J019v27n02_06 
Sacker, A. y Cable, N. (2006). Do adolescent leisure-time physical activities foster health and web-being in adulthood? Evidence from two British birth cohorts. European Journal of Public Health, 16, 3, pp. 331-335. http:// dx.doi.org/10.1093/eurpub/cki 189 Torres, G.; Carpio, E.; Lara, A.J y Zagalaz, M.J (2014). Niveles de condición física de escolares de educación primaria en relación a su nivel de actividad física y al género. Retos: nuevas tendencias en educación física, deporte y recreación, 25, 17-22.

Ussher, M.; Owen, C. G.; Cook, D. y Whincup, P. (2007). The relationship between physical activity, sedentary behavior and psychological wellbeing among adolescents. Social Psychiatry and Psychiatry Epidemiology, 42, pp. 851-856. http://dx. doi.org/10.1007/ s00127-007-0232-x

MAGDALENA SÁENZ DE JUBERA OCÓN (Universidad de La Rioja-España). Código ORCID: 0000-0001-8086-907X

Doctora en Ciencias de la Educación por la Universidad de Alcalá y Licenciada en Pedagogía por la Universidad de Navarra. Profesora en la Facultad de Letras y de la Educación de la Universidad de La Rioja. Investigadora en temas de Educación, Orientación para la carrera y Ocio, autora de varias publicaciones sobre ocio y educación, competencia digital docente, propuestas organizativas y de educación inclusiva que contribuyan a la mejora de la práctica educativa.

EVA SANZ ARAZURI (Universidad de La Rioja-España)

Código ORCID: 0000-0003-3035-8751

Doctora por la Universidad de Zaragoza, Profesora Contratada Doctora de la Facultad de Letras y de la Educación en la Universidad de La Rioja. Miembro del equipo de investigación Actividad Física y Deporte en el espacio y tiempo de Ocio (AFYDO). Miembro de la Red de Equipos de Investigación en Estudios de Ocio, OcioGune. Investigadora en temas de Educación, Ocio, Familia, Valores y Actividad Física, 10 investigaciones de I+D+I financiadas, 10 tesis doctorales dirigidas, autora de 5 libros 58 artículos indexados y capítulos de libro.

EMAUELE ISIDORI (Universidad Foro Itálico de Roma-Italia). Código ORCID: 000-002-5214-6015

Doctor en actividad física y salud por la Universidad Alfonso X y en Ciencias de la Educación por la Universidad di Perugia. Profesor titular de Pedagogía general, social y del deporte en la Universidad de Roma "Foro Itálico", donde dirige el Laboratorio de Pedagogía General. Es autor de numerosos libros y publicaciones en inglés, español e italiano en temas de pedagogía y filosofía de la educación deportiva.

Recibido: 05/04/2017

Aceptado: 16/09/2017 
NOTAS DE INVESTIGACIÓN RESEARCH NOTES 



\title{
LA POTENCIA ESTADÍSTICA PROSPECTIVA DEL VOTO RETROSPECTIVO EN ESPAÑA (1996-2015) \\ THE PROSPECTIVE STATISTICAL POWER OF THE RETROSPECTIVE VOTE IN SPAIN (1996-2015)
}

\author{
Antonio Alaminos \\ Universidad de Alicante, España \\ Alaminos@ua.es \\ Paloma Alaminos-Fernández \\ Universidad de Alicante, España \\ Paloma.Alaminos.fernandez@gmail.con
}

\begin{abstract}
Cómo citar / Citation
Alaminos, Antonio y Alaminos-Fernández, Paloma (2017) "La potencia estadística prospectiva del voto retrospectivo en España (1996 a 2015)". OBETS. Revista de Ciencias Sociales, 12(Extra 1): pp-pp. 249-278. doi:10.14198/OBETS2017.12.1.20

\section{Resumen $^{1}$}

Esta investigación evalúa la capacidad explicativa y predictiva que la valoración de la actuación/gestión del PP y el PSOE tiene sobre los resultados electorales de estos partidos. La valoración de los dos partidos se efectúa de forma anticipada, entre uno y dos meses antes de las elecciones. El diseño incluye conjuntamente ambos partidos, por lo que la hipótesis testada es acerca de la relación entre valoración de la actuación y gestión de los partidos y sus resultados electorales (Downs, 1957). Es decir, la eficacia del modelo de voto retrospectivo para explicar y anticipar los resultados electorales en elecciones generales en España. Las convocatorias estudiadas son las elecciones generales en España del 1996, 2000, 2004, 2008, 2011 y 2015. Los datos de valoración pro-
\end{abstract}

\footnotetext{
${ }^{1}$ La investigación fue efectuada durante una estancia de investigación en 2017 del profesor Antonio Alaminos en el GESIS - Leibniz-Institute for the Social Sciences, financiada con la ayuda PRX16/00176, del programa de movilidad Salvador de Madariaga del Ministerio de Educación, Cultura y Deporte.
} 
ceden del Centro de Investigaciones Sociológicas y los resultados electorales del Ministerio del Interior. Tras testar las hipótesis de asociación y de dependencia entre el voto retrospectivo anticipado y los resultados electorales, se concluye que el indicador de valoración de la actuación/gestión del PP y el PSOE tiene, con carácter anticipado de uno a dos meses, una asociación y capacidad explicativa significativa de los resultados electorales. La potencia predictiva, por el contrario, no es limitada al considerar por razones de tamaño muestral en el mismo modelo los resultados de los dos partidos. Asimismo, se efectúa un análisis de la potencia estadística de varios modelos, comparando diferentes especificaciones.

Palabras clave: voto retrospectivo, prospectiva electoral, PP, PSOE, resultados electorales, poder estadístico

\section{Abstract}

This research evaluates the explanatory and predictive capacity that the evaluation of the political performance / management of the PP and the PSOE has on the electoral results of these political parties. The assessment of the two political parties is made in advance, between one and two months before the elections. The design includes both political parties, so the hypothesis tested is about the relationship between performance appraisal and governmental party management with their election results (Downs, 1957). That is, the effectiveness of the retrospective voting model to explain and anticipate electoral results in general elections in Spain. The calls for papers are the general elections in Spain in 1996, 2000, 2004, 2008, 2011 and 2015. The evaluation data come from the Centre for Sociological Research and the election results of the Ministry of the Interior. After testing the hypotheses of association and dependence between the anticipated retrospective voting and the electoral results, it is concluded that the performance / management indicator of the PP and the PSOE has, in advance of one to two months, an association and significant explanatory power of electoral results. The predictive power, on the other hand, is low. Due to the sample size, the two political parties are included in the same model. Also, an analysis of the statistical power of several models is carried out, comparing different specifications.

Keywords: retrospective vote, electoral forecast, PP, PSOE, elections results, statistical power

\section{Extended Summary}

This research, evaluates the explicative and predictive capacity that the evaluation of the performance / management of the PP and the PSOE has had on the electoral results of these political parties. This assessment of the two political parties is measured in advance, between one and two months before the elections. The design includes the two political parties together, so the hypothesis tested is about the relationship between performance appraisal and their election results (Downs, 1957). That is, the effectiveness of the retrospective voting model to anticipate election results. The data refers to the general elections in Spain in 1996, 2000, 2004, 2008, 2011 and 2015. The public 
opinion about the performance of the political parties come from the social surveys implemented by the CIS and the election results of the Ministry of the Interior.

Before analysing the hypotheses regarding the association, dependence and prospective capacity of the political parties' evaluation with their electoral results, several analyses have been carried out to verify the validity of merging the data coming from the two political parties.

Two main issues need to be addressed. First, whether there are significant differences in the results or assessments of political parties depending on whether they are in government at that time. Second, if these differences exist or not, controlling for the political party that is valued. That is, whether the results or assessment depends on the political party being considered or that it is in the government or not. If the relationship between the performance of political parties and their electoral results are independent of the political party or whether it occupies the government or not, the two political parties can be integrated in the same analysis.

To test the possibility of data integration, two complementary analysis strategies have been applied. In the first place, it has been verified that there is a significant partial correlation between the evaluation of the political parties and their electoral results, controlling for whether the political party is at that moment or not in the government. The same analysis is applied by controlling for the political party (PP or PSOE). To confirm that the analysis is independent of the statistical method employed, we did a variance analysis taking as control variables if the political party occupy the government or not, as well as the political party evaluated.

After conducting the analyses, the conclusion is that there are no significant statistical differences between the assessments received by a political party or its election results, when the political party is on the government or in the opposition. The same conclusion is reached by testing the ratings received by a political party or its election results, according to the political party.

The analysis by partial correlation corroborates the two previous analyses, leading to the same conclusion: it is viable methodologically to merge the data coming from the two political parties to estimate a single model.

For all the tests carried out in this research the calculation of its statistical power has been made. This additional control makes it possible to take account of type I and type II errors together. Given the sample size, with very small number of cases, it is essential to know and control the two types of errors. As reference to fix the power of the analyses has adopted the convention of a of .05 and a of .20. In that sense, the reference power is .80 or higher.

The ecological fallacy has been indirectly controlled by a proxy variable, the intention to vote. Other studies (Alaminos and Alaminos-Fernández) have established a statistically significant relationship between the intention to vote and the assessment of the political parties considered, in several social surveys. Also, since an indicator of the performance of the political parties is used, the possible impact on their value of the lost cases has been evaluated. Over a 20year period, a trend of increasing or decreasing missing values may affect the 
magnitude of the indicator, reducing its significance to a subpopulation. This control of the indicator has been carried out in other studies, showing a stable level with an average value of less than $5 \%$.

In the analysis of the data, four different models have been tested, varying in the sample size. To the extent that each case is an election, excluding cases involves excluding elections from analysis. The different excluded elections correspond to atypical results, due to exceptional circumstances. For example, the terrorist attacks of 2011 and the lack of transparency of the conservative government produced an evident effect on the electoral conjuncture. Among the conclusions, the withdrawal of the analysis of atypical elections produces a better fit of the models, increasing the statistical coefficients of association or dependence between the assessment of the performance of the political parties and their electoral results.

After testing the hypotheses of association and dependence between the anticipated retrospective evaluation of the political parties and their electoral results, it is concluded that the PP / PSOE performance indicator shows in advance, from one to two months, an association and significant predictive power of electoral results. Also, an analysis of the statistical power of several models is carried out, comparing different specifications.

Although there is an explanatory capacity (regression model) of retrospective voting, it lacks sufficient precision to define an electoral prediction. It correctly shows the voting tendency, and allows to estimate an average change of $0.41 \%$ in the election results for each increase of a point in the valuation indicator. But the confidence interval and error is excessive to produce an adequate forecast.

\section{INTRODUCCIÓN}

El empleo de series temporales con la finalidad de predecir los resultados electorales es una técnica empleada profusamente, en especial en las elecciones de Estados Unidos y Gran Bretaña. En la ciencia política continental, la predicción en general y la aplicación de series en particular, tiene una presencia y un status científico menor. En el empleo de series, las funciones de popularidad, eficacia económica, así como con otros factores explicativos del voto; se utilizan como variables anticipatorias de los resultados electorales. En el otro lado de la ecuación, se utilizan como variables dependientes tanto los resultados electorales como las intenciones de voto procedentes de encuestas. Los modelos que forman parte de los métodos orientados a la prospectiva electoral encuentran para el mundo anglosajón, antecedentes evidentes en Lewis-Beck y Rice (1992), o Campbell y Garand (2000).

Sin embargo, la prospectiva electoral continúa siendo una actividad atípica en el mundo académico, con una mayor producción de modelos explicativos o descriptivos que no extrapolan al futuro los resultados de sus análisis. De 
hecho, la prospectiva electoral es en algunos países es una actividad poco apreciada por la academia (Norpoth y Gschwend, 2010). En parte, es comprensible por el riesgo para el prestigio del investigador, quien al efectuar una predicción pone en riesgo la calidad y capacidad explicativa de su modelo. Desde el método científico, pocas cosas son tan definitivas para contradecir un modelo explicativo como su incapacidad para estimar el futuro. En ese sentido, es paradójico que las explicaciones se limiten al presente y al pasado, no exponiéndose a extrapolar las consecuencias futuras de lo propuesto. A menos que se trate de "modelos forenses" orientados a explicar la historia.

No obstante, lo cierto es que, si la ciencia social es realmente ciencia, el conocimiento adecuado de las condiciones de decisión de voto implica su testado mediante la conocida dinámica de diagnóstico, predicción y comprobación de resultados de la previsión. Es el corazón metodológico del experimento, en la medida que todo experimento se basa en que a partir de establecer unas condiciones (diseño experimental) se propone un resultado (hipótesis, pero también predicción) para posteriormente contrastar los resultados con lo esperado. Un buen modelo explicativo del comportamiento electoral debe finalizar, si se habla de ciencia, en una propuesta de predicción que incluya una previsión de error. Es en dicho proceso de explicación, predicción, error y aprendizaje del error donde se encuentra el refinamiento del conocimiento. La realidad es siempre el mejor test que un modelo electoral científico pueda tener (Alaminos, 1994).

Un modelo importante en la predicción y explicación electoral es el denominado voto retrospectivo o, más coloquialmente, interpretado en su versión como voto de castigo. Así, una valoración positiva de la actuación del gobierno le concede ventaja en la contienda electoral y viceversa; una valoración negativa da más oportunidades de competir a los partidos de la oposición. Desde un punto de vista cognitivo, este modelo de voto propone que el cálculo electoral efectuado por los votantes se apoya más en la evaluación del pasado que en las promesas para el futuro ${ }^{2}$. El modelo de voto retrospectivo es aplicable a los demás partidos de la oposición y no exclusivamente al partido con responsabilidad de gobierno en ese momento. Downs (1957) considera que la evaluación retrospectiva incluye necesariamente a los partidos de oposición con experiencia de gobierno. Para valorar un partido se tiene en cuenta también lo hecho y dicho en el pasado (en definitiva, la fiabilidad de los partidos) por todos ellos. Así, en el voto retrospectivo se estaría considerado la valoración de la ges-

2 Para un desarrollo más exhaustivo de este modelo ver Alaminos y Alaminos-Fernández, 2017. 
tión/actuación de los partidos en la actualidad, pero también lo realizado en pasados gobiernos. En ese sentido, el voto retrospectivo como factor explicativo es aplicable a los partidos con experiencia de gobierno, tanto en la actualidad como en el pasado.

La serie de datos de opinión valorando la actuación o gestión de los partidos mayoritarios comienza en 1996. En esta investigación emplearemos las series de los dos partidos con experiencia de gobierno en España desde 1996: el Partido Socialista Obrero Español y el Partido Popular. No existen datos para los gobiernos de UCD, en lo que se refiere a la valoración de su acción de gobierno hasta 1982.

Como se ha podido apreciar, la estrategia de análisis explicativo-prospectivo mediante series temporales, cuando se intenta utilizar los resultados de las elecciones, encuentra una limitación evidente en el número de casos. Para España, a diferencias de otras democracias, las series temporales de resultados electorales son bastante limitadas. En especial si se pretende analizar, como se ha advertido, en relación a datos procedentes de encuestas de opinión pública. En España la base de datos de opinión pública más importante es el Centro de Investigaciones Sociológicas. Los datos de este centro, por lo general, mantiene una importante consistencia en los fraseos y cierres de sus preguntas, permitiendo series temporales prolongadas. No obstante, los datos disponibles comienzan en 1996 con una medición de valoración para el PSOE en enero. Esta valoración se anticipa a las elecciones de marzo de ese mismo año, pudiendo emplearse como indicador anticipado. Con carácter general a los dos partidos, las series de valoración comienzan en diciembre de 1996. Por ello utilizar las series de valoración de actividad de los partidos como indicador asociado o anticipado de los resultados electorales (incluyendo los datos de los dos partidos) remite a las elecciones generales de 2000 como primer punto común en la serie, seguidos de 2004, 2008, 2011, 2015 y 2016.

Sin embargo, dado que en esta investigación se va a estudiar el voto retrospectivo a partir de la valoración de la gestión/actuación de los partidos PP y PSOE, las elecciones de 2016 no pueden ser analizadas dado que las encuestas del CIS no preguntaron durante este periodo de gobierno en funciones por el indicador. Este hecho limita a cinco las elecciones cuyos resultados son analizables para los dos partidos. El límite objetivo que representa el número de elecciones hace inviable la estrategia de análisis mediante series temporales independientes para cada partido. No obstante, la hipótesis de relación entre el voto retrospectivo anticipado (valoración de actuación/gestión del partido dos meses antes de las elecciones) y los resultados electorales puede ser sometida a prueba con estrategias alternativas. Dos de ellas, las empleadas en esta inves- 
tigación son el análisis de correlación bivariable y el análisis de regresión simple. Por ello, en este diseño, vamos a proceder al testado y análisis de la hipótesis sobre la asociación entre la valoración de la actuación o gestión del Partido Popular y del PSOE (según estuviesen o no en el gobierno) y sus resultados electorales. Precisamente el pequeño tamaño muestral hace especialmente pertinente el estudio de la potencia estadística de los análisis, por ello para cada uno de los modelos se ha efectuado un estudio de su potencia estadística.

\section{DISEÑO DE LA INVESTIGACIÓN}

En este estudio se toma como medición de la valoración de la actividad o gestión del partido el indicador desarrollado por el Centro de Investigaciones Sociológicas, a partir de barómetros sucesivos. Estos indicadores y su cálculo están explicados en su página web³. Las preguntas empleadas para estos indicadores son dos, reformuladas según el partido en el gobierno o en la oposición, y se incluyen en los barómetros del primer mes para cada trimestre desde 1996. Está redactada del siguiente modo "En su conjunto, ¿cómo calificaría Ud. la gestión que está haciendo el gobierno del PSOE?" o en su caso, "En su conjunto, ¿cómo calificaría Ud. la gestión que está haciendo el gobierno del PP?" dependiendo del partido que ocupa el gobierno en ese momento. La misma lógica se aplica para el partido que en esa ocasión se encuentre en la oposición, "En general, ¿cómo calificaría Ud. la actuación política que está teniendo el PSOE en la oposición? o "En general, ¿ cómo calificaría Ud. la actuación política que está teniendo el PP en la oposición?". En definitiva, dos preguntas que, según el partido en el gobierno u oposición, cambia "gestión" por "actuación" el objeto de valoración. Todas ellas ofrecen las mismas opciones de respuesta: "muy buena", "buena", "regular", "mala" o "muy mala".

A partir de estas dos preguntas, se construye el indicador mediante una combinación ponderada de los porcentajes de respuesta a cada valoración ("muy buena", "buena", "regular", "mala" o "muy mala"). Son dos indicadores calculados de forma idéntica salvo por la pregunta que les da origen, según se evalúe "gestión" (Valoración de la Labor del Gobierno, ILG) o "actuación" (Valoración de la labor del Primer Partido de la Oposición, ILO). En el caso español, dada la alternancia establecida hasta ahora entre dos partidos en el gobierno (PSOE y PP), al evaluar el comportamiento del gobierno y la oposición se están evaluando de hecho a los dos partidos.

\footnotetext{
${ }^{3}$ http://www.cis.es/cis/opencms/ES/11_barometros/metodologia.html\#ISPGO
} 


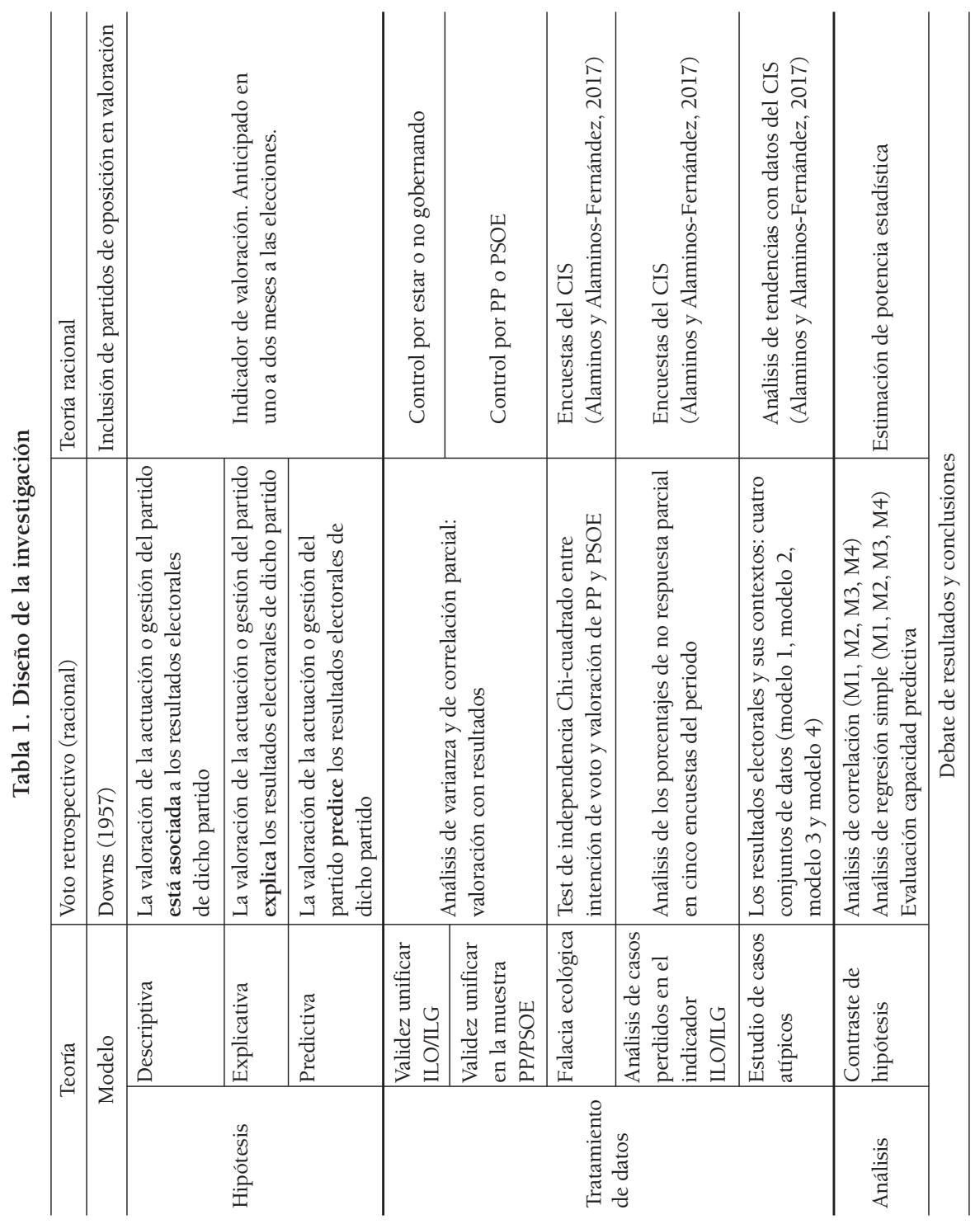


Ambos indicadores ponderan las proporciones de cada categoría, con 100 para la valoración "Muy buena" hasta 0 para la categoría "Muy mala". Posteriormente dividen por la suma de las proporciones para normalizar el rango de variación entre 0 y 100 . Valores próximos a cero expresan una mala valoración del partido (gestión/actuación), un rechazo absoluto a la gestión o actuación del partido. Valores próximos a 100 indican lo contrario, una excelente valoración. De hecho, 100 significa que toda la población evalúa su gestión o actuación de forma excelente. Según la formulación propuesta por el Centro de Investigaciones Sociológicas:

- Indicador de la Labor del Gobierno (ILG):

$$
I L G=\frac{100 \cdot \rho_{1}+75 \cdot \rho_{2}+50 \cdot \rho_{3}+25 \cdot \rho_{4}+0 . \rho_{5}}{\rho_{1}+\rho_{2}+\rho_{3}+\rho_{4}+\rho_{5}}
$$

- Indicador de la Labor de la Oposición (ILO):

$$
I L O=\frac{100 \cdot \rho_{1}+75 \cdot \rho_{2}+50 \cdot \rho_{3}+25 \cdot \rho_{4}+0 \cdot \rho_{5}}{\rho_{1}+\rho_{2}+\rho_{3}+\rho_{4}+\rho_{5}}
$$

En esta investigación hemos empleado de forma indistinta los dos indicadores dentro del mismo modelo. De acuerdo con Downs (1957), en la valoración actual de la actuación o gestión de ambos partidos están incluidas la opinión sobre sus actuaciones o gestiones pasadas (según gobernaran o no). Por ello, la valoración retrospectiva del PP o del PSOE se refieren tanto a su actuación (desde la oposición) como su gestión (como gobierno).

La intercambiabilidad empírica de dichos indicadores como medición de la actuación o gestión de un mismo partido, ya esté en el gobierno u oposición, es un presupuesto que requiere una evaluación empírica específica que efectuaremos más adelante.

Las estructuras de los datos no permiten su tratamiento como serie temporal, pero sí la aplicación de procedimientos que los considere como mediciones puntuales de dos características. Es el caso del análisis de correlación o el de regresión simple. Esto no significa que la característica de temporalidad desaparezca del análisis. En los dos análisis se comparan dos variables desplazadas temporalmente, en la medida que la opinión pública que valora la actuación o gestión de los dos partidos políticos se realiza entre uno y dos meses antes de las elecciones. La dimensión temporal aparece de forma integrada en los datos, dado que las mediciones de valoración de gestión o actuación están efectuadas de forma anticipada uno o dos meses antes de las elecciones. Es interesante destacar que para cuatro de las elecciones la valoración utilizada ha sido medida aproximadamente dos meses antes, siendo la de 2011 realizada en el mes anterior (octubre). 
Tabla 1. Resultados electorales y valoración de actuación/gestión del PP y PSOE

\begin{tabular}{|c|c|c|c|c|}
\hline Fechas & $\begin{array}{l}\text { Resultado } \\
\text { electoral PP }\end{array}$ & $\begin{array}{l}\text { Indicador } \\
\text { actuación/ } \\
\text { gestión PP }\end{array}$ & $\begin{array}{c}\text { Resultado } \\
\text { electoral PSOE }\end{array}$ & $\begin{array}{c}\text { Indicador } \\
\text { actuación/ } \\
\text { gestión PSOE }\end{array}$ \\
\hline Enero 1996 & & & & 40,00 \\
\hline Marzo 1996 & 29,90 & & 29,00 & \\
\hline Enero 2000 & & 58,60 & & 46,00 \\
\hline Marzo 2000 & 30,40 & & 23,30 & \\
\hline Enero 2004 & & 46,60 & & 43,30 \\
\hline Marzo 2004 & 28,20 & & 31,90 & \\
\hline Enero 2008 & & 37,00 & & 49,10 \\
\hline Marzo 2008 & 29,30 & & 32,20 & \\
\hline Octubre 2011 & & 30,60 & & 25,70 \\
\hline Noviembre 2011 & 30,40 & & 19,60 & \\
\hline Octubre 2015 & & 30,50 & & 33,60 \\
\hline Diciembre 2015 & 19,80 & & 15,20 & \\
\hline Junio 2016 & 21,70 & & 14,90 & \\
\hline
\end{tabular}

Fuente: elaboración propia con datos del CIS y resultados electorales en las elecciones generales según Ministerio del Interior

Para poder integrar en un mismo análisis los datos procedentes de los dos partidos deben resolverse dos cuestiones principales. Primero, si existe o no diferencias significativas en los resultados o en las valoraciones de los partidos políticos dependiendo de si están o no ocupando el gobierno en ese momento. Segundo, si esas diferencias existen controladas por el partido político que es valorado, es decir, si los resultados o valoración dependen del partido que está siendo considerado o de que este en el gobierno o no. En el caso de que la relación entre valoración de la actuación de los partidos políticos, al igual que sus resultados electorales, sean independientes del partido político o de si ocupa el gobierno o no, se pueden integrar en el mismo análisis los dos partidos, así como los dos fraseos diferentes según se esté en el gobierno o no.

Para testar la posibilidad de integración de los datos, se han aplicado dos estrategias de análisis complementarias. En primer lugar, se ha comprobado que existe correlación parcial significativa entre la evaluación de los partidos políticos y sus resultados electorales, controlando si el partido político está en 
ese momento o no en el gobierno. El mismo análisis es aplicado controlando si el partido político es PP o PSOE. Finalmente, para confirmar que el análisis es independiente del método estadístico empleado, se ha efectuado un contraste de medias de valoración y de resultados electorales tomando como variables de control el ocupar o no el gobierno, así como el partido político evaluado.

\section{ANÁLISIS}

En una investigación que testa una hipótesis de relación entre dos variables cuando el número de casos es muy limitado es esencial considerar la potencia (poder) estadística. Dado que no se dispone de datos para todas las elecciones, realmente se está operando sobre un muestreo temporal de ellas.

En ese sentido, en un diseño alternativo, la presencia de asociación puede darse por cierta si consideramos las elecciones analizadas exclusivamente en sí mismas, sin pretensión de generalización. No se requiere significación alguna si estudiamos las elecciones generales de 2000 a 2015.

Sin embargo, el diseño de esta investigación tiene aspiraciones de generalización y llegado el caso, predicción del pasado (de poder acceder a datos) o del futuro. El diseño empleado conduce, por lo tanto, a la necesidad de testar la significación estadística de los coeficientes estimados, en tanto que los casos utilizados se entienden como una muestra temporal y no como población.

En el proceso de testar la hipótesis nula "no existe relación entre la valoración anticipada y los resultados electorales de los partidos", con una muestra pequeña, procede tener en cuenta los dos tipos de errores, de tipo I y de tipo II.

El error tipo I, (error de tipo $\alpha$ o falso positivo), es el error que se comete al rechazar la hipótesis nula cuando esta es verdadera. En nuestro caso, un ejemplo es afirmar que sí existe relación cuando realmente no la hay. Es decir, se rechaza la hipótesis nula de que no existe relación. El control de referencia del error de tipo I es la significación del test, como se sabe, convencionalmente establecida en el 0.01 o el 0.05 .

El error de tipo II (error tipo $\beta$ o falso negativo), se produce cuando como resultado del test no se rechaza la hipótesis nula, siendo la hipótesis nula falsa en la población. Es decir, en nuestro caso, se afirma que no hay relación entre las dos variables consideradas cuando realmente sí existe. El error $\beta$ se puede producir por causas muy diversas, en especial cuando el tamaño muestral es muy bajo o el tamaño de los efectos (una asociación, por ejemplo) analizados muy débil. Recuérdese que cuando los efectos son débiles, por lo general se requiere una muestra más elevada para que se aprecien empíricamente en un test estadístico. En este tipo de error tiene un peso especial el tamaño muestral o la magnitud del efecto considerado. 
En relación con estos dos tipos de errores se emplea el concepto de potencia o poder estadístico de un test. El poder es expresado de forma simple, como la probabilidad de detectar un efecto cuando realmente existe un efecto para ser detectado. El poder de un test expresa el equilibrio entre los errores de tipo I y de tipo II.

Se calcula substrayendo a 1 el valor $\beta$, poder $=(1-\beta)$. Según Cohen (1992), un valor $\beta$ de referencia para el error de tipo II es de 0.20 lo que significa que el poder mínimo de referencia utilizado convencionalmente es de .80 . Análisis con un poder de .80 o superior son especialmente confiables respecto al efecto detectado en el análisis.

En definitiva, en nuestro caso si aceptamos que existe relación estadística entre valoración anticipada y resultado electoral (rechazamos la hipótesis nula), cuando en la realidad esa relación no existe cometemos un error de tipo I. Si, por el contrario, cuando afirmamos que no existe relación entre valoración anticipada y resultado electoral (no rechazamos la hipótesis nula) cuando realmente sí existe, nos encontramos ante un error de tipo II.

Ambos errores se encuentran relacionados (cuando se incrementa la probabilidad de cometer uno desciende la probabilidad de cometer el otro), por lo que se debe buscar un equilibrio entre ellos. Convencionalmente (Cohen, 1992) se considera que el equilibrio adecuado se encuentra con un alfa de 0,05 y una beta de 0,20. Aplicando estos criterios como referencia, se propone un intercambio de cuatro-a-uno entre la $\beta$ (error de tipo II) y la $\alpha$ (error de tipo I). No obstante, el criterio de referencia depende de los objetivos del investigador. Así, en un análisis clínico, por lo general es preferible minimizar los errores de tipo II (falso negativo), aún a riesgo de incrementar los errores de tipo I (falso positivo). En definitiva, es mejor seguir buscando y comprobando que no existe un tumor cancerígeno que decidir rápidamente que no existe (falso negativo, error de tipo II o $\beta$ ).

El cálculo del poder de los test que vamos a realizar es especialmente relevante en este caso, con un número reducido de observaciones. En la medida que no existe la posibilidad de incrementar el número de observaciones, su uso es de carácter "post hoc", para determinar la potencia del análisis una vez realizado, así como para evaluar las $\alpha$, dado que en nuestro caso, tenemos interés en determinar la potencia que tendríamos cuando apliquemos el cálculo de la correlación, entre los resultados electorales en las elecciones generales de España (1996 a 2015), y la valoración de la actuación/gestión del Partido Popular y del Partido Socialista. Para el análisis de regresión simple se usará la variable anticipada de valoración como independiente. 
En definitiva, el poder expresa el grado de probabilidad de rechazar mediante un test estadístico la $\mathrm{H}_{0}$ cuando es realmente falsa. Dado que por lo general el interés de los investigadores está dirigido a mostrar que la hipótesis alternativa (que sí hay relación) es correcta, indirectamente la potencia habla de la validez de los resultados del test.

En el cálculo del poder, se calibran los test mediante la distribución muestral del estadístico en el caso de la hipótesis nula. Por lo general, la distribución muestral sigue una distribución normal o de chi-cuadrado. Esta distribución de referencia permite calcular los "valores críticos": aquellos valores que excedan el valor crítico se consideran demasiado elevados para lo que se esperaría si la hipótesis nula fuera cierta. En otras palabras, el test superaría el riesgo de cometer un error de tipo II. Asimismo, si el test estadístico genera una distribución normal estándar para el caso de la hipótesis nula, la alternativa a la hipótesis nula genera una distribución normal con media diferente de cero. Esta media es conocida como el parámetro de "no centralidad".

Para todos los test efectuados en esta investigación se ha efectuado el cálculo de su potencia o poder estadístico. Este control adicional permite tener en cuenta conjuntamente los errores de tipo I y de tipo II. Dado el tamaño muestral, con número de casos muy pequeño, es esencial conocer y controlar los dos tipos de errores. Como referencia para fijar la potencia de los análisis se ha adoptado la convención de un $\alpha$ de .005 y una $\beta$ de .20. En ese sentido, la potencia de referencia es de .80 o superior.

Una cuestión interesante es evaluar hasta qué punto es consistente integrar en una misma variable las valoraciones cuando se trata de actuación (partido en la oposición) o gestión (partido en el gobierno). En principio, responde a dos operativizaciones diferenciadas en su fraseo, y con ello dos variables diferentes. Sin embargo, como hemos considerado, Downs (1957) y de forma muy próxima al concepto imagen de partido, propone que en la valoración actual de un partido se toma en consideración sus actuaciones en conjunto, tanto cuando estaban en el gobierno como en la oposición. Es el enfoque adoptado en otros estudios previos (Alaminos y Alaminos-Fernández, 2017).

Para determinar hasta que punto la evaluación del partido varía según esté en el gobierno o en la oposición, se ha efectuado un control de correlación incorporando dicha información. El análisis de correlación parcial entre la valoración anticipada y los resultados se mantiene significativa para el modelo 1. La correlación parcial entre la valoración anticipada de la actuación o gestión de los partidos y su resultado electoral controlada por estar en gobierno u oposición es de .56, con un $\alpha$ significativa al .04 (una cola) con 8 grados de libertad. En este caso, al efectuar el control por una tercera variable el número de casos se convierte en un elemento clave a efectos de significación. 
Gráfico 1. Poder estadístico del análisis de correlación parcial entre la valoración anticipada y resultados electorales controlada por estar o no en el gobierno

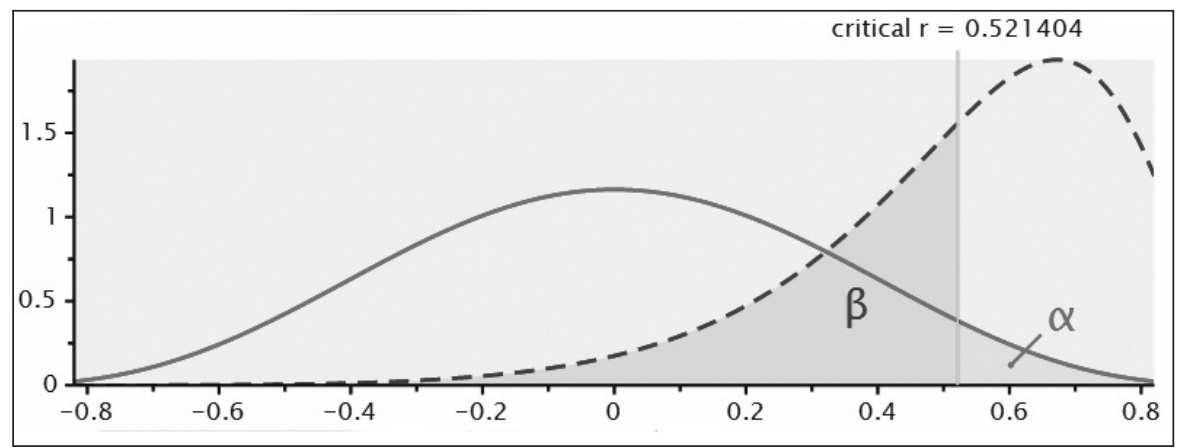

Fuente: elaboración propia con GPower

El resultado permite rechazar la hipótesis nula (no correlación) y además el coeficiente de correlación parcial estimado es de .56 (y por lo tanto superior al valor crítico estimado por el modelo, de .52), si bien el poder del test es de 60 .

Asimismo, se efectúa un análisis de varianza, comparando las medias del indicador de valoración de gestión o de actuación, así como de los resultados electorales, según se haya estado en el gobierno o la oposición en el momento de la convocatoria electoral. Se concluye que las dos variables (ILO e ILG) no

Gráfico 2. Poder estadístico del análisis de varianza: valoración anticipada controlada por estar o no en el gobierno

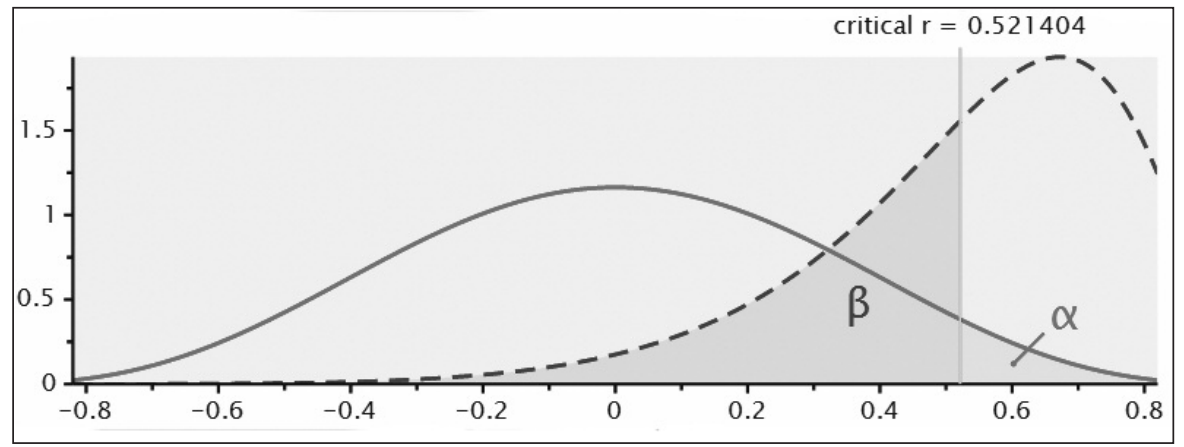

Fuente: elaboración propia con GPower 
muestran diferencias significativas para el partido según esté en el gobierno o fuera de él. El gráfico 2, se muestra el análisis del poder estadístico del test efectuado para contrastar la diferencia de medias para el caso del indicador anticipado de valoración.

\section{Gráfico 3. Poder estadístico del análisis de varianza: resultados electorales controlados por estar o no en el gobierno}

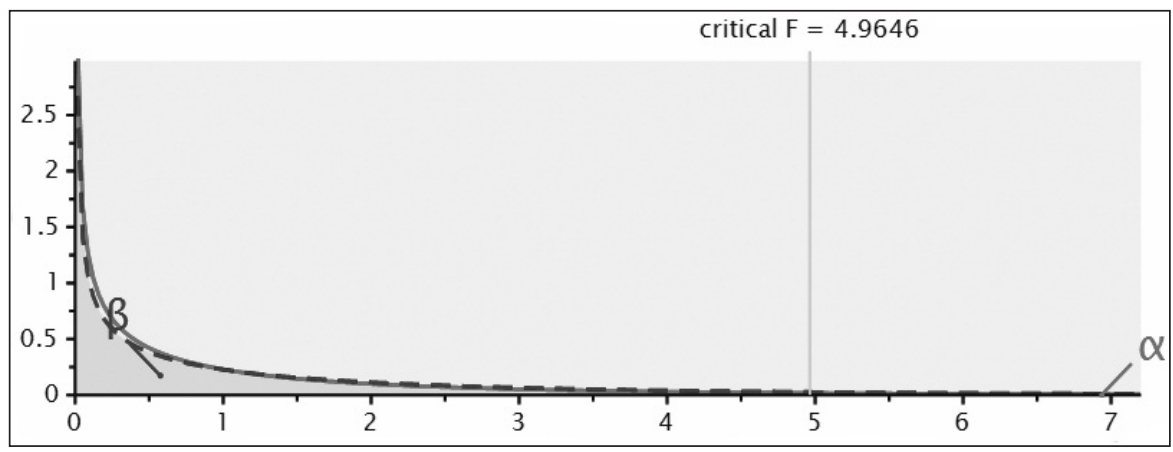

Fuente: elaboración propia con GPower

Con un valor F de .35 (valor crítico de 5.31) y una significación de .57 para un poder estimado del test de 0.16 , es muy improbable que se trate de un falso negativo. El gráfico 3 muestra el resultado para el test de contraste de medias en los resultados electorales según el partido ocupe o no el gobierno en el momento de la convocatoria electoral.

Es un resultado equivalente al hallado para el contraste de medias anterior. Con un valor F de .18 (crítico de 4.9) y para una significación de 0.68; el test ofrece un poder estadístico de 0.08 . La conclusión es que es muy improbable que se trate de un falso negativo. Expresado en otras palabras, se acepta la hipótesis nula que afirma "las diferencias de medias no son significativas" con bastante certeza estadística.

En los dos enfoques los resultados son consistentes. No se puede rechazar, con los datos disponibles (en base al análisis de varianza de las dos variables controlando por estar en gobierno u oposición), la $\mathrm{H}_{0}$ que afirma que no existen diferencias entre las medias de valoración anticipada o resultados electorales; según se esté en el gobierno u oposición.

Del mismo modo, en base al análisis de correlación parcial, se puede rechazar la $\mathrm{H}_{0}$ que afirma que no existe relación entre valoración anticipada y resultados cuando se controla por estar en el gobierno u oposición. 
Gráfico 4. Poder estadístico del análisis de correlación parcial entre la valoración anticipada y resultados electorales controlada por el partido político (PP y PSOE)

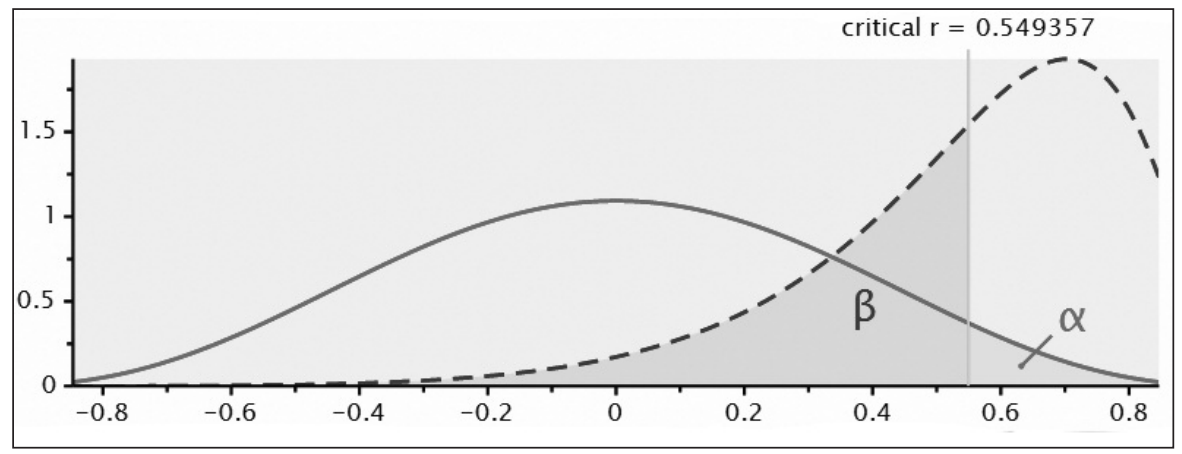

Fuente: elaboración propia con GPower

La conclusión que resulta de las dos formulaciones de la pregunta es la misma, independiente del test estadístico utilizado: no existe heterogeneidad incorporada en los datos procedente de que un partido político esté valorado en ILO o ILG. No hay varianza significativa en las variables (valoración y resultados) procedente de que el partido se encuentre en función de gestión o actuación.

Una situación semejante se reproduce cuando el control se realiza según de qué partido se trate: el PP o el PSOE. En otras palabras, ¿se mantiene la relación entre los resultados y la valoración cuando se controla por el efecto del partido al que corresponde dichas valoraciones y resultados? Nuevamente, el análisis indica que los datos del PP y los del PSOE pueden ser incorporados conjuntamente al mismo análisis, dado que no aportan una heterogeneidad específica. La correlación parcial es de .58 con una significación de .04 y una potencia de 0.59 . La correlación es mayor que el valor crítico propuesto por el análisis de potencia (0.54). En ese sentido, se puede rechazar la hipótesis de que la correlación entre valoración y gestión esté condicionada por el partido. En una estrategia alternativa, se procede a comparar las medias en las dos variables utilizadas en el análisis, según los datos procedan del PP o del PSOE. La hipótesis nula plantea que no existe diferencias entre las medias de valoración y resultados correspondientes a cada partido. Nuevamente, el contraste de medias entre los dos partidos, las variables referidas a la valoración de la gestión o actuación de los partidos y sus resultados electorales; indican que no se puede rechazar la hipótesis nula: no hay diferencias significativas estadísticamente. 
Gráfico 5. Poder estadístico del análisis de varianza: valoración anticipada controlada por el partido político (PP y PSOE)

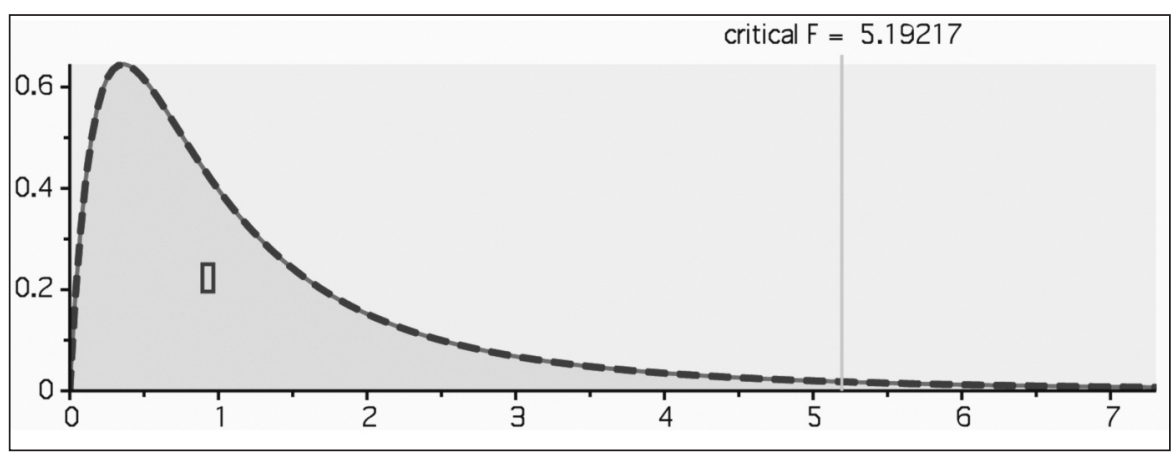

Fuente: elaboración propia con GPower

Gráfico 6. Poder estadístico del análisis de varianza: resultados electorales controlados por el partido político (PP y PSOE)

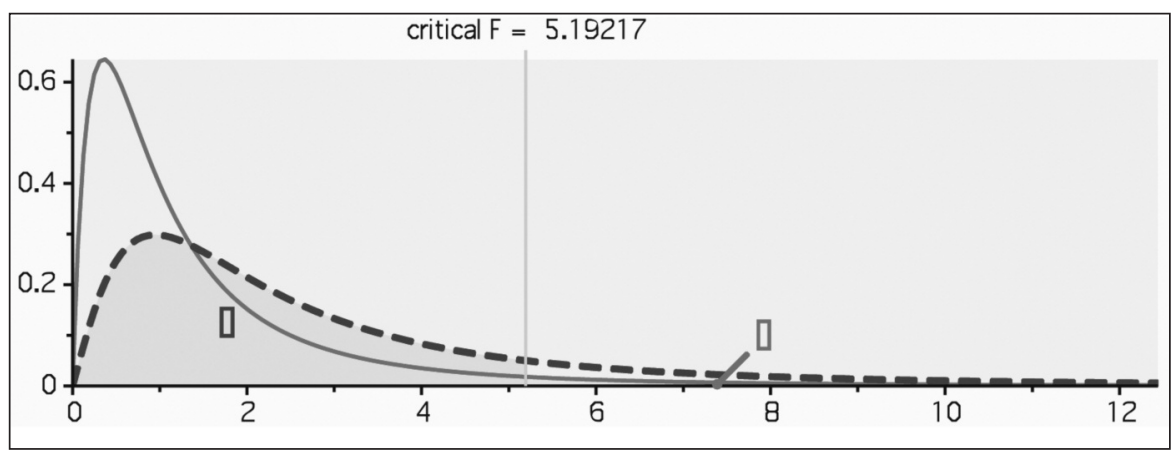

Fuente: elaboración propia con GPower

Las diferencias en las medias de valoración de la actuación o gestión controladas por los dos partidos ofrecen una F de .02 (muy inferior al valor crítico) y una significación de .87 (potencia de 0.05). El contraste de medias para el caso de los resultados electorales, una F de .66 (también muy inferior al valor crítico) y con una significación del .44 (potencia 0.17). Para las dos variables no se puede rechazar la hipótesis de que no existe diferencias en las medias según partido. Los análisis anteriores permiten incluir los datos procedentes de los dos partidos políticos, PP y PSOE, en un mismo análisis a efectos de testar las hipótesis establecidas.

En resumen, después de efectuar los análisis, la conclusión es que no existen diferencias estadísticas significativas entre las valoraciones que recibe un 
partido político o sus resultados electorales, según se encuentre o no gobernando. Idéntica conclusión se alcanza en relación a ambos partidos al testar las valoraciones que recibe cada uno o sus resultados electorales.

El análisis mediante correlación parcial corrobora los dos análisis anteriores, conduciendo a la misma conclusión: es viable metodológicamente fusionar los datos procedentes de los dos partidos a efectos de estimar un único modelo.

\section{ANÁLISIS DE RELACIÓN}

En el análisis se han empleado los valores para el PP y el PSOE conjuntamente. Esto impide estimar un coeficiente para cada partido. La hipótesis alternativa $\left(\mathrm{H}_{1}\right)$, en ese sentido, se formula del siguiente modo: "existe una relación entre la valoración anticipada (entre uno y dos meses) de los dos partidos mayoritarios, PP y PSOE, y sus resultados electorales". En definitiva, la relación entre valoración de la actuación/gestión y resultado electoral, indistintamente del partido. En ese sentido, se está testando la eficacia explicativa del voto retrospectivo respecto a los resultados electorales del partido en el gobierno y del principal partido de la oposición (Downs, 1957).

Vamos seguidamente a calcular cuatro análisis de correlación diferentes según el número de casos y el criterio de inclusión.

(1) El modelo 1, con una muestra (n) de 11 casos, incluye toda la información disponible, incluyendo la valoración al PSOE en 1996.

(2) El modelo 2, reduce el tamaño muestral a 10, excluyendo del análisis el caso de 1996. En este análisis el período temporal considerado es del 2000 a 2015.

(3) El modelo 3, reduce el número de casos a 9, incluyendo la valoración del PSOE en 1996, pero excluyendo del análisis dos elecciones "atípicas". Es el caso de los resultados del PSOE en 2004 y del Partido Popular en el 2011. Los resultados de estos dos partidos en esas elecciones reflejan condiciones excepcionales, considerando la tendencia general que experimentaban ambos partidos (Alaminos y Alaminos-Fernández, 2017).

(4) El modelo 4 con 8 casos, equivale al modelo 3, suprimiendo el valor del PSOE para 1996.

Para cada uno de los test de correlación se ha calculado su poder, para evaluar completamente las condiciones del análisis y sus resultados. Como sabemos, un test estadístico puede producir resultados no significativos cuando la hipótesis nula es correcta (y realmente no hay efecto), pero también cuando el test aplicado (por razones de tamaño muestral, por ejemplo) no ha sido lo bastante potente para establecer la validez de la hipótesis alternativa (existencia de relación) frente a la hipótesis nula. 
Cuando no se conoce el poder del test aplicado, es imposible diferenciar entre las dos situaciones que pueden dar lugar a unos resultados no significativos. En este análisis conocemos el tamaño muestral (n), la magnitud del efecto, y el alfa utilizado, por lo que se procede a determinar el poder (1- $\beta$ ) de cada uno de los test efectuados. Por ello, la estimación del poder de estos test está orientado a realizar una evaluación crítica de la magnitud del error tipo II ( $\beta$ ) aceptando la hipótesis nula de no efecto. Y por el contario, confirmando la existencia del efecto considerado.

La definición de parámetro de no centralidad de la distribución de t-test de la correlación entre dos variables es:

$$
\delta=\sqrt{\frac{\rho^{2}}{1-\rho^{2}}} \cdot \sqrt{N}
$$

Siendo $\rho$ la correlación de la población asociada con la $\mathrm{H}_{1}$ y $\mathrm{N}$ el tamaño muestral. Este parámetro refleja el grado en que $\mathrm{H}_{0}$ es falsa (Johnson y Kotz, 1970).

El tipo de análisis efectuado es de correlación (t-test) a una cola debido a que la hipótesis que queremos testar $\mathrm{H}_{0}$ "la correlación es menor o igual a cero" frente a la hipótesis alternativa $\mathrm{H}_{1}$ "la correlación es mayor que cero".

Lo primero que puede observarse es que las correlaciones para los cuatro modelos son significativas por debajo del 0.05. En otras palabras, se rechaza la hipótesis $\mathrm{H}_{0}$ "no existe relación entre ambas variables". La valoración anticipada (uno a dos meses antes de las elecciones) está relacionada con los resultados que obtiene el partido en las elecciones generales.

Tabla 2. Correlación entre la valoración de actuación/gestión y resultado electoral del PP y PSOE

\begin{tabular}{l|c|c|c|c|c}
\hline & (n) & $\begin{array}{c}\text { Correlación } r \\
\text { (valoración actuación/gestión } \\
\text { y resultado electoral del PP } \\
\text { y PSOE) }\end{array}$ & Sig. & $\begin{array}{c}\text { Potencia } \\
(\alpha=.05)\end{array}$ & $\begin{array}{c}\text { Valor } \\
\text { crítico } r\end{array}$ \\
\hline Modelo 1 & 11 & .57 & .03 & .61 & .52 \\
\hline Modelo 2 & 10 & .57 & .04 & .57 & .54 \\
\hline Modelo 3 & 9 & .72 & .01 & .80 & .58 \\
\hline Modelo 4 & 8 & .75 & .01 & .77 & .62 \\
\hline
\end{tabular}

Fuente: elaboración propia. Test de significación a una cola 
Dada la relación entre el número de casos y la magnitud del efecto y potencia, la potencia del análisis es mayor en los modelos 3 y 4 a pesar de tener un número menor de muestra. Estos modelos excluyen los resultados de PSOE en 2004 y PP en 2011, por lo que confirman el carácter excepcional de estos resultados en la pauta normal de ambos partidos. Cuando se retiran del análisis, mejora la punta de relación entre valoración anticipada de la actuación del partido y sus resultados electorales. Asimismo, la exclusión de los resultados del PSOE en 1996 (analizando los resultados desde las elecciones de 2000 a 2015), aun reduciendo el tamaño muestral, incrementa la relación entre ambas variables (valoración de actuación/gestión y resultados electorales); posiblemente en la medida que las elecciones de 2000 iniciaron un ciclo electoral diferenciado del ciclo anterior 1989-1996, y con ello una relación más consistente entre valoración de estos partidos y su resultado.

El análisis del poder del test actúa aquí con finalidad de refinamiento, dado que, en definitiva; el poder expresa el grado de probabilidad de rechazar me-

Gráfico 7. Análisis del poder. Modelos de correlación

Modelo 1

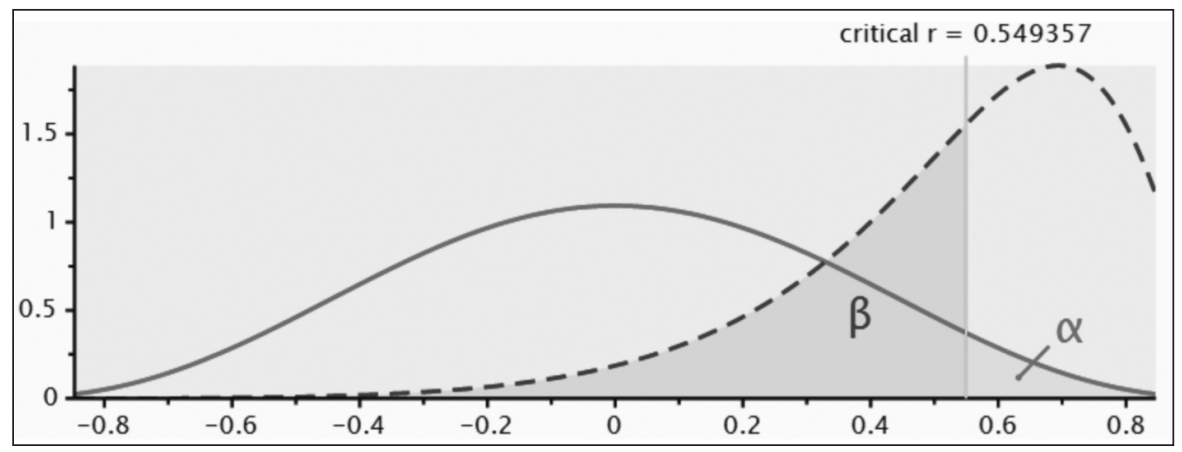

Modelo 2

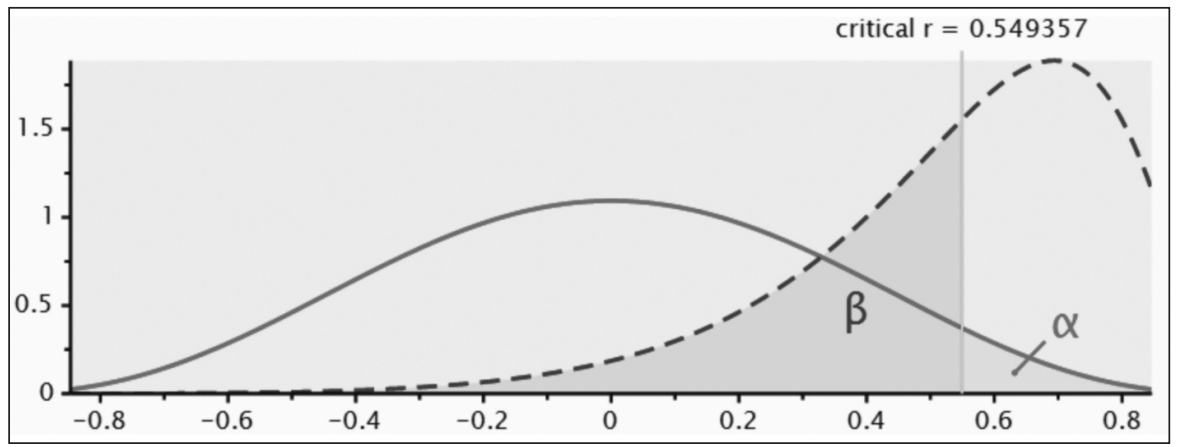


Modelo 3

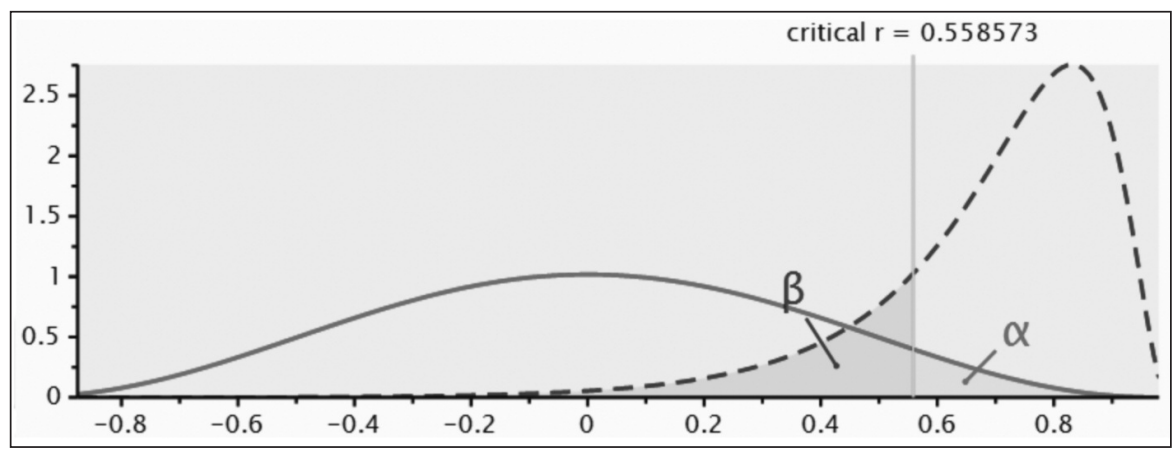

Modelo 4

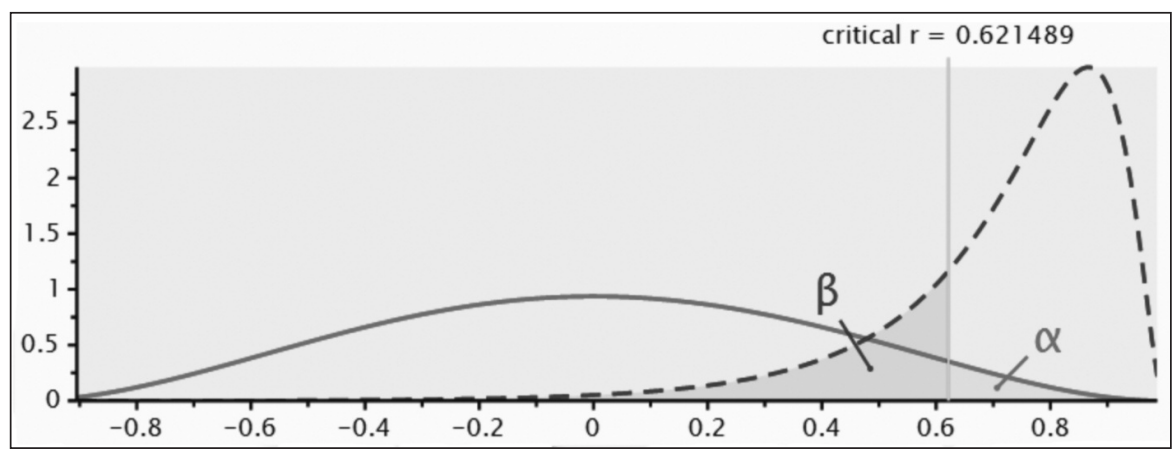

Fuente: elaboración propia. Programa GPower

diante un test estadístico la $\mathrm{H}_{0}$ cuando es realmente falsa. Todos los test afirman la existencia de correlación entre las variables (rechazo de $\mathrm{H}_{0}$ ), si bien con mayor probabilidad en los modelos 3 y 4 . La correlación en el modelo 3 es de .72 y en el 4 de .75 , ambos con una significación de .01, muy elevada. De todos los modelos, el más consistente en términos de poder es el modelo 3.

La comprobación de los valores críticos (los valores que excedan el valor crítico se consideran demasiado elevados para lo que se esperaría si la hipótesis nula fuera cierta) confirma que todas las correlaciones superan los valores críticos. En otras palabras, el test superaría el riesgo de cometer un error de tipo II (falso negativo). Los gráficos siguientes muestran la distribución centrada del estadístico para $\mathrm{H}_{0}$, y la distribución no centrada asociada para la $\mathrm{H}_{1}$. 
Sin embargo, un elemento importante es la falacia ecológica. Al tratar con variables e indicadores de carácter agregado. Para controlar la falacia del agregado corresponde establecer si esta relación agregada se establece a nivel micro mediante las respuestas individuales en la encuesta. Es decir, ¿confianza y voto se relacionan en las respuestas individuales? Los controles efectuados a nivel micro (Alaminos y Alaminos-Fernández, 2017) muestran una relación significativa entre la intención de voto y la valoración de la actuación o gestión del PP y del PSOE. Es decir, se valida que la relación entre ambas variables se establece a nivel individual, controlando con ello la falacia ecológica o del agregado. Ciertamente, no es lo mismo la intención de voto que voto efectivo en unas elecciones. Sin embargo, esta estrategia de validación proxy es la única viable, dado el carácter secreto del voto.

\section{ANÁLISIS DE DEPENDENCIA}

Tras confirmar la validez general de la hipótesis por la que la valoración de la actuación/gestión de los partidos está relacionada (anticipadamente) con los resultados electorales de estos partidos, vamos a proceder a efectuar un análisis de regresión simple, estableciendo una relación de dependencia entre los resultados electorales y la valoración anticipada en el tiempo de estos partidos.

Evidentemente es un ejercicio que no permite por su diseño establecer una predicción específica para cada uno de los dos partidos, siendo considerados de forma indistinta. Esto incrementa la heterogeneidad en la relación, por lo que previsiblemente la capacidad predictiva (al ser conjunta para los dos partidos) sea menor de la que podría encontrarse de ser los datos diferenciados para cada uno de los partidos.

$$
y=\mathrm{a}+b \mathrm{x}+e
$$

Siendo $y$ los resultados electorales expresados en porcentaje del Partido Popular y del Partido Socialista en las elecciones generales de 1996, 2000, 2004, 2008, 2011 y 2015; y x el indicador de la valoración que la opinión pública tiene de la actuación (ILO) o la gestión (ILG) del Partido Popular o del Partido Socialista Obrero Español.

En el análisis de regresión se van a testar los cuatro modelos considerados para el análisis de correlación. Es decir, variando el tamaño muestral y con ello la especificación del modelo, en lo que se refiere al número de elecciones o de partidos incluidos en el modelo.

Vamos seguidamente a calcular los cuatro análisis de regresión diferentes según el número de casos y el criterio de inclusión. 
Tabla 3. Regresión simple de la valoración actuación/gestión de los partidos (x) y su resultado electoral (y)

\begin{tabular}{l|c|c|c|c|c|c|c}
\hline & & $\mathrm{R}^{2}$ & $\mathrm{~B}$ & $t$ & & & \\
\hline $\mathrm{Y}=\mathrm{a}+\mathrm{bx}+\mathrm{e}$ & $(\mathrm{n})$ & & & & Sig. & $\begin{array}{c}\text { Potencia } \\
(\alpha=.05)\end{array}$ & $\begin{array}{c}\text { Valor } \\
\text { crítico t }\end{array}$ \\
\hline Modelo 1 & 11 & .32 &, 570 & 2,083 &, 067 & .68 & 1.8 \\
\hline Modelo 2 & 10 & .33 &, 578 & 2,002 &, 080 & .64 & 1.8 \\
\hline Modelo 3 & 9 & .52 &, 722 & 2,757 &, 028 & .87 & 1.8 \\
\hline Modelo 4 & 8 & .56 &, 751 & 2,782 &, 032 & .88 & 1.9 \\
\hline
\end{tabular}

Fuente: elaboración propia. Poder calculado con el programa GPower

(1) El modelo 1, con una muestra (n) de 11 casos, contiene toda la información disponible, incluyendo la valoración al PSOE en 1996.

(2) El modelo 2, reduce el tamaño muestral a 10, excluyendo del análisis el caso de 1996. En este análisis el período temporal considerado es de 2000 a 2015.

(3) El modelo 3, reduce el número de casos a 9, incluyendo la valoración del PSOE en 1996, pero excluyendo del análisis dos elecciones "atípicas". Es el caso de los resultados del PSOE en 2004 y del Partido Popular en el 2011.

(4) El modelo 4 con 8 casos, excluye las elecciones atípicas de 2004 para el PSOE, de 2011 para el Partido Popular y suprime el valor del PSOE para 1996.

En la tabla 3 se muestran varios indicadores para los cuatro modelos de regresión testados.

Dos de los modelos, el 1 y el 2, son los que muestran peor ajuste. Sus coeficientes $\beta$ tienen una significación superior al 0.05 por lo que en este caso no puede rechazarse la hipótesis nula. Es decir, que los resultados electorales no dependen de la valoración de la actuación/gestión de los partidos efectuada de forma anticipada. Sin embargo, el poder de los dos análisis es bastante elevado. El modelo 1, con una significación de 0.06 , tiene un poder de .68, mientras que el modelo 2, con significación de 0.08 un poder .64. En ambos análisis no se rechaza la hipótesis nula, (que afirma la no dependencia), sin embargo, las potencias que muestran son muy elevadas y las simulaciones de análisis de sensibilidad efectuadas indican como ligeros cambios en la magnitud del coeficiente $\beta$ o un incremento del tamaño muestral dan por resultado el rechazo de la hipótesis nula, y por lo tanto afirmando dependencia. En ese sentido, la aceptación de la hipótesis nula, que establece que no existe relación de dependencia entre ambas variables, tiene asociada una probabilidad elevada de ser rechazada (como posible error de tipo II). 
Los modelos 3 y 4, como sabemos, analizan menos casos (una $n$ menor que los otros dos modelos) pero se benefician de la eliminación de los casos atípicos (PSOE en 2004 y PP en 2011) y del cambio de ciclo electoral. Sus

\section{Gráfico 8. Análisis del poder. Modelos de regresión}

Modelo 1

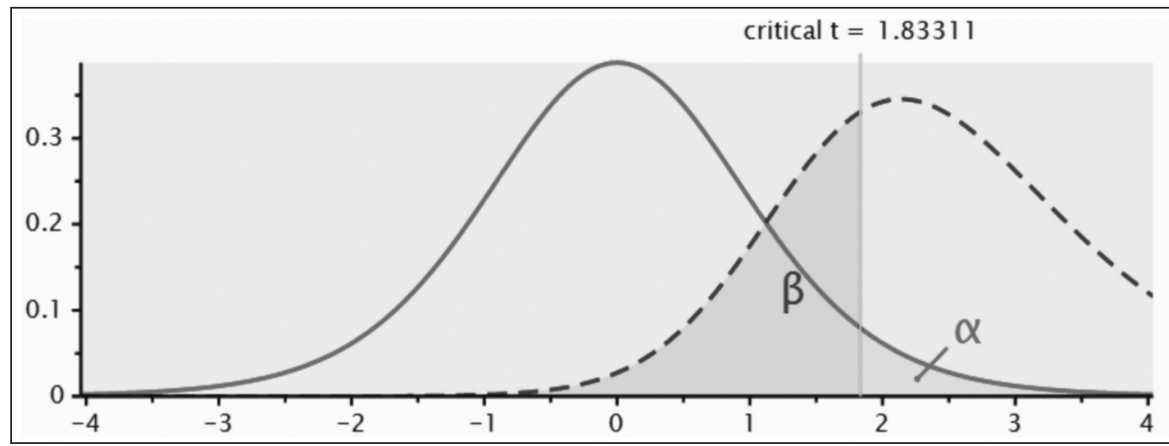

Modelo 2

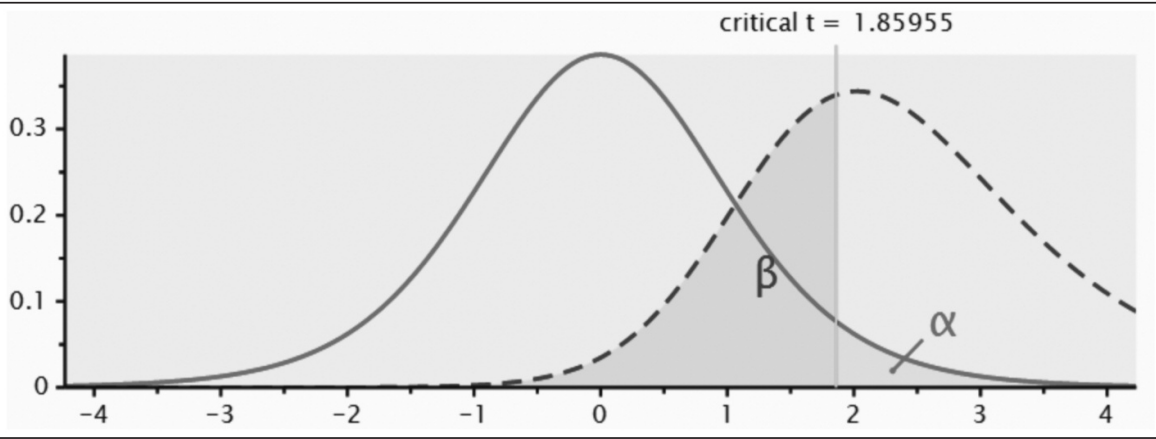

Modelo 3

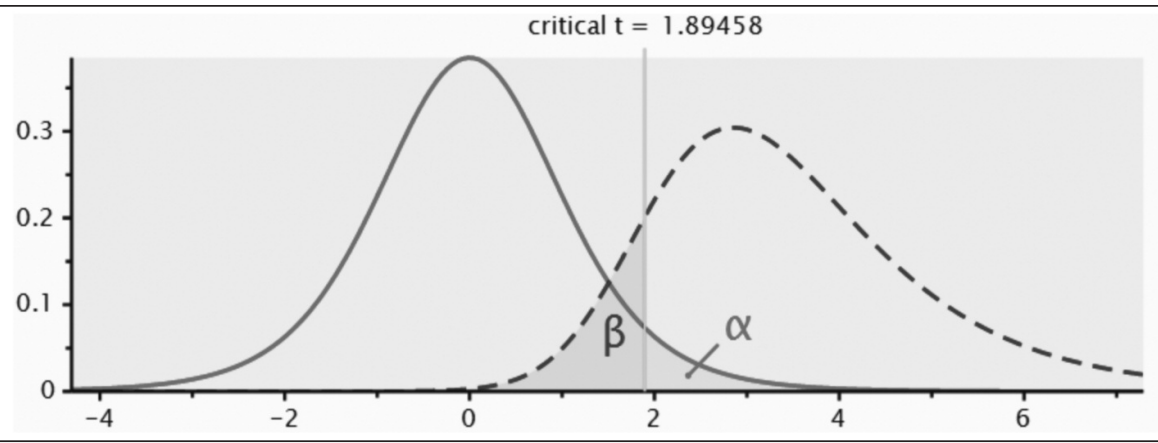




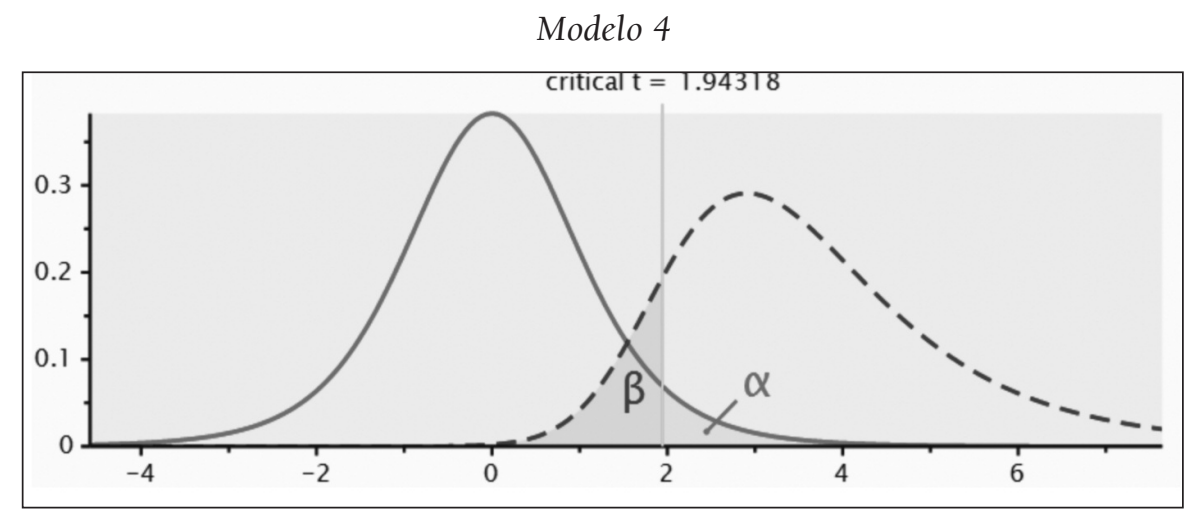

Fuente: elaboración propia. Programa GPower

coeficientes de significación $\alpha$ son inferiores al 0.05 permitiendo con ello rechazar la $\mathrm{H}_{0}$. Aceptando, con ello, la hipótesis alternativa $\left(\mathrm{H}_{1}\right)$ y afirmando que sí existe relación de dependencia entre los resultados electorales y la valoración de la gestión/actuación del PP y del PSOE. El modelo 3 tiene una significación $\alpha$ del 0.02 , con una potencia de .87. El modelo 4, una significación de 0.03 con una potencia de .88. En ambos casos se puede rechazar la hipótesis nula de una forma bastante concluyente.

Para los análisis que excluyen las elecciones atípicas, se concluye que la valoración anticipada de la actuación y gestión de los partidos influye en los resultados electorales de los dos partidos. Una valoración anticipada medida entre uno y dos meses antes de las elecciones. En ese sentido, es un modelo donde la variable dependiente, por su operativización, se anticipa a los resultados electorales.

Considerando el coeficiente de determinación $\mathrm{R}^{2}$, en el caso del modelo 3 la valoración de la gestión/actuación explica el 52\% de la varianza de los resultados electorales del PP y del PSOE. El modelo 4, explica el 56\% de la variación en los resultados electorales. Considerando que se trata de una sola variable de opinión (valoración), medida con carácter anticipado a las elecciones; puede concluirse que tiene un valor explicativo muy elevado.

En ese sentido, si bien el valor explicativo es elevado, su capacidad predictiva es más limitada. Consideremos el modelo 3, por ejemplo, que conserva el mayor número de casos para una potencia superior a .80. En el gráfico puede apreciarse la estructura de relación lineal presente en los datos. No obstante, se observa como la distancia de varios de los puntos a la recta de regresión es demasiado elevada. Esta distancia expresa el error entre los casos predichos (su valor se encuentra dentro de la recta) y los reales. Apreciándose una estructura rela- 
Gráfico 9. Modelo 3. Regresión entre valoración anticipada y resultados electorales del PP y del PSOE

\section{Regresión entre valoración anticipada y resultados electorales}

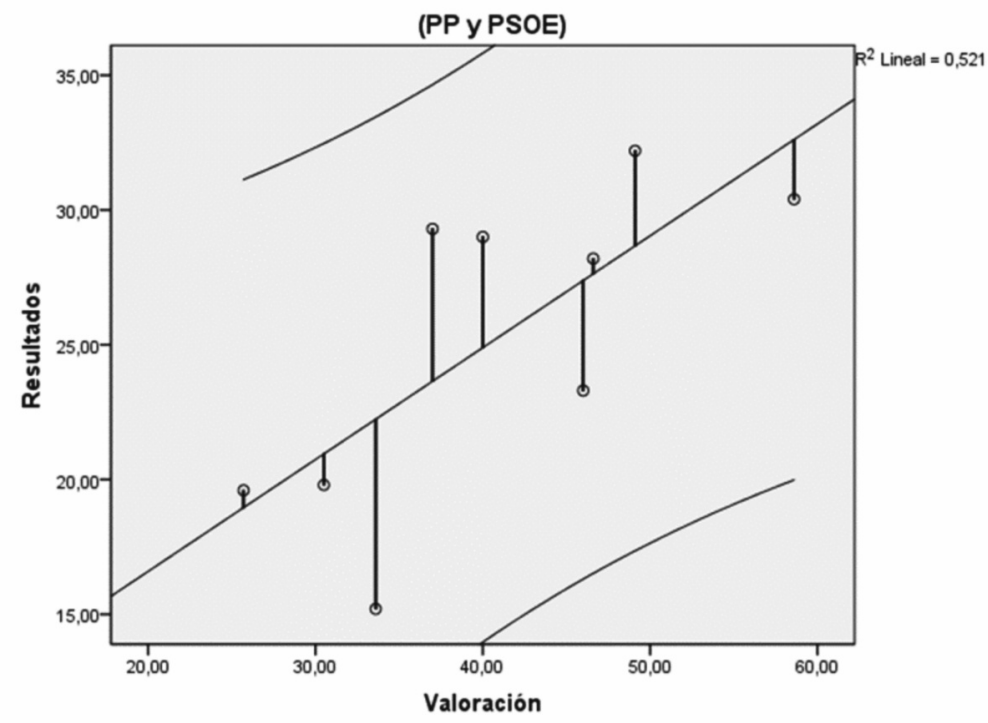

Fuente: elaboración propia

Gráfico 10. Estimación del poder, correlación de valoración anticipada y resultados del Partido Popular

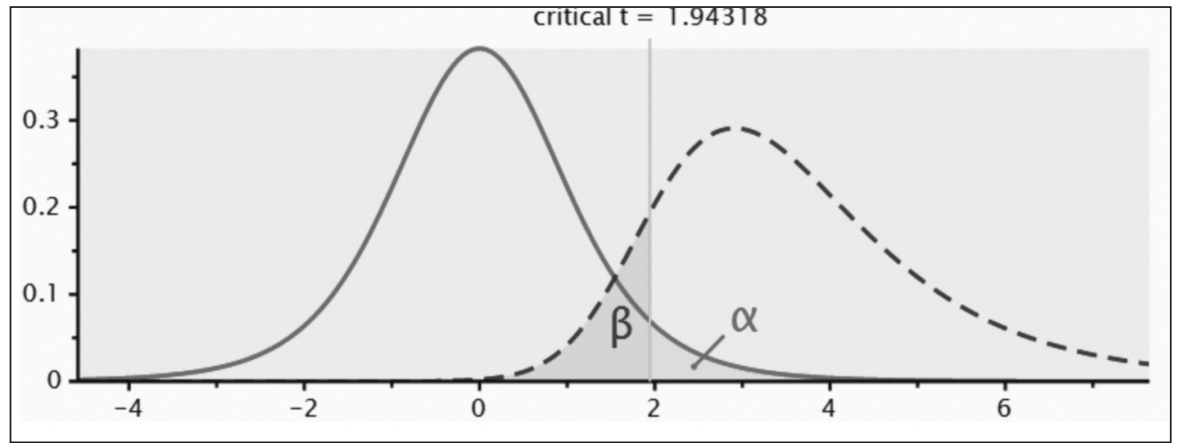

Fuente: elaboración propia con el programa GPower 
cional explicativa de forma lineal, la capacidad predictiva es menor por esa excesiva distancia de varios puntos (resultados electorales del PP y del PSOE) a la recta.

Considerando el valor B no estandarizado de los dos modelos con suficiente significación, (modelo 3 y modelo 4) podemos evaluar el impacto electoral de la valoración de la actuación y gestión. En ambos modelos se establece que el cambio medio esperado en los resultados electorales del PP y del PSOE es de un incremento del .41 por cada punto de mejora en el indicador de valoración de actuación o gestión. En otras palabras, que la mejora en la valoración tiene consecuencias en los resultados electorales casi de medio punto de voto por cada incremento de un punto en el indicador de valoración.

\section{DEBATE DE RESULTADOS}

El análisis, debido a su diseño, evalúa la relación entre el indicador anticipado de valoración de la actuación y gestión de los partidos mayoritarios y sus resultados electorales. Los resultados de los dos partidos son tratados de forma indistinta (no se considera qué resultado o valoración corresponde con cada partido). Por este motivo, los análisis realizados concluyen que existe dicha relación, pero debido al diseño no existe la posibilidad de efectuar predicciones de voto, o considerar el cambio medio en resultados según evaluación para cada uno de los partidos por separado.

El número de casos para un único partido, máximo cinco casos, además de incluir elecciones atípicas, no permite un análisis adecuado. Así, por ejemplo, el poder de un análisis de correlación de la valoración del PP únicamente con los resultados electorales del PP es de 0.16 . No es viable un análisis separado para cada partido, como ejemplifica el gráfico 10.

No obstante, se confirma la relación entre confianza y voto final en términos agregados. Es decir, que existe una relación estadísticamente significativa entre el indicador de valoración de la actuación o gestión de los partidos considerados (PP y PSOE) con los resultados electorales obtenidos en la elección general más próxima a la última valoración (entre uno y dos meses se anticipa la valoración a la elección).

La falacia ecológica ha sido controlada indirectamente mediante una variable proxy, la intención de voto. En otros estudios (Alaminos y Alaminos-Fernández) se ha establecido una relación estadísticamente significativa entre la intención de voto y la valoración de los partidos políticos considerados.

Asimismo, dado que se utiliza un indicador de valoración de la actuación de los partidos, se ha evaluado el posible impacto en su valor de los casos per- 
didos. En un período temporal de 20 años, una tendencia de incremento o decremento en los valores perdidos puede afectar a la magnitud del indicador, reduciendo su significado a una subpoblación. Este control del indicador se ha efectuado en otros estudios, mostrando un nivel estable con un valor promedio inferior al 5\%.

En el análisis de los datos se han testado cuatro modelos diferentes, variando en el tamaño muestral. En la medida que cada caso es una elección, excluir casos implica excluir elecciones del análisis. Las diferentes elecciones excluidas corresponden con resultados atípicos, consecuencia de circunstancias excepcionales. Por ejemplo, los ataques terroristas de 2011 y la falta de transparencia del gobierno conservador produjeron un efecto evidente en las tendencias electorales. Entre las conclusiones, la retirada del análisis de las elecciones atípicas produce un mejor ajuste de los modelos, incrementando los coeficientes estadísticos de asociación o dependencia entre valoración de la actuación de los partidos y sus resultados electorales.

Esta asociación puede expresarse como relación de dependencia, en la que se propone que el resultado electoral vendría explicado en parte por la valoración que la sociedad hace de la actuación o gestión de los partidos que han tenido desde 1996 responsabilidades de gobierno. Los análisis efectuados confirman, para el caso de los modelos 3 y 4 (con eliminación de resultados atípicos) que el indicador de valoración anticipado explica porcentajes superiores al $50 \%$ de la variable explicada. En definitiva, de acuerdo a los postulados de la teoría del voto retrospectivo.

Asimismo, nuevamente se valida como determinadas elecciones tienen un resultado atípico dentro del modelo de voto retrospectivo, incrementándose la capacidad explicativa de este tipo de voto cuando se retiran de la ecuación.

Desde el punto de vista metodológico, los test realizados muestran que, de acuerdo a Downs, en la práctica la diferencia entre la valoración de la actuación o de la gestión según el partido esté o no gobernando no es significativa. En otras palabras, que, para España, la valoración del partido incorpora las actuaciones o gestiones efectuada anteriormente; de tal forma que las valoraciones de las capacidades del partido parecen haberse incorporado como una parte significativa de lo que se denomina "imagen de partido". La crisis actual del sistema de bipartidismo efectivo es, en ese sentido, un reflejo de la acción acumulada de una valoración negativa de la opinión pública que se ha incorporado a sus imágenes de partido.

Por último, si bien existe una capacidad explicativa (modelo de regresión) del voto retrospectivo, esta carece de la precisión suficiente para definir 
una predicción electoral. Muestra correctamente la tendencia de voto y permite estimar un cambio medio de $0,41 \%$ en los resultados electorales por cada incremento de un punto en el indicador de valoración, pero el intervalo de confianza y el error es excesivo para ser adecuado.

\section{BIBLIOGRAFÍA}

Alaminos, A. (2015) "Forecasting elections with high volatility". Italian Journal of Applied Statistics. 25 (2), 165-184.

Alaminos, A. (2012) "Missing mixed mode: elemental structures". OBETS: Revista de Ciencias Sociales. Número 7. Páginas 327-333

Alaminos, A. (1996) "Las predicciones electorales de las elecciones generales de 1996 en España”. En VVAA. Elecciones Generales 1996 y medios de comunicación en la región de Murcia. Murcia: Fundación Instituto de la Comunicación de Murcia

Alaminos, A. (1994). "Predicciones electorales en España" en Alaminos et al., Future Days. Alicante: Fundación Cultural CAM.

Alaminos, A. (1991). Teoría y práctica de la encuesta. Madrid: CEDEAL

Alaminos, A. y Alaminos Fernández, P. (2017). Las dinámicas del voto retrospectivo en España (1996-2017). Tendencias Sociales. Revista de Sociología. Aceptada pendiente de publicación.

Alaminos, A., Francés, F., Penalva, C., Santacreu, O. (2015). Análisis multivariante para las Ciencias Sociales I. Índices de distancia, conglomerados y análisis factorial. Cuenca (Ecuador): PYDLOS

Bono, R. y J. Arnaud, 1995. Consideraciones generales en torno a los estudios de potencia. Anales de psicología. 11.193 -202.

Bradley, D.R., Russell, R.L. y Reeve, C.P. (1996) Statistical power in complex experimental designs. Behavior Research Methods, Instruments \& Computers, 28, 319-326

Campbell y Garand (2000) Before the vote. Forecasting American national elections. Thousand Oaks, CA: Sage Publications.

Cohen, J. (1992). A power primer. Psychological Bulletin. 112. 155-159

Cohen, J. (1988) Statistical power analysis for the behavioral sciences. Hillsdale: Lawrence Erlbaum Associates.

Downs (1957). An economic theory of democracy. New York: Harper and Row.

Ellis, Paul D. (2010). The Essential Guide to Effect Sizes: An Introduction to Statistical Power, Meta-Analysis and the Interpretation of Research Results. United Kingdom: Cambridge University Press.

Faul, F., Erdfelder, E., Lang, A.-G., y Buchner, A. (2007). G*Power 3: A flexible statistical power analysis program for the social, behavioral, and biomedical sciences. Behavior Research Methods, 39, 175-191.

Faul, F., Erdfelder, E., Buchner, A., y Lang, A.-G. (2009). Statistical power analyses using $G^{*}$ Power 3.1: Tests for correlation and regression analyses. Behavior Research Methods, 41, 1149-1160. 
Frances, J.F, Alaminos, A., Penalva, C., Santacreu, O. (2014) El proceso de medición de la realidad social: La investigación a través de encuestas. Cuenca (Ecuador): PYDLOS Johnson, N. y Kotz, S. (1970) Continuous Univariate Distributions: Distributions in Statistics. Vol 1. Boston. Hougton Mifflin

Lewis-Beck y Rice (1992) Forecasting elections. Washington DC: CQ Press.

Norpoth, Helmut; Gschwend, Thomas (2010). The chancellor model: forecasting German elections. International Journal of Forecasting 26, 1, 42-53 pages.

DOI: http://dx.doi.org/doi:10.1016/j.ijforecast.2009.02.008

ANTONIO ALAMINOS es Catedrático de Sociología. Especializado en métodos de investigación y modelos matemáticos. Líneas de investigación en comportamiento electoral, contacto cultural y prospectiva.

PALOMA ALAMINOS es investigadora del programa Música y Sociedad. Especializada en métodos de investigación aplicada a los impactos sociales de las Nuevas Tecnologías, prospectiva y movimientos sociales. Su última publicación, Las dinámicas del voto retrospectivo en España en la revistaTendencias.

Recibido: 09/09/2017

Aceptado: 03/10/2017 
CRÍTICA DE LIBROS BOOK REVIEWS 



\title{
Crítica de libro: \\ FRANCISCO JOSÉ FRANCÉS GARCÍA, ANTONIO ALAMINOS CHICA, CLEMENTE PENALVA VERDÚ, ÓSCAR ANTONIO SANTACREU FERNÁNDEZ
} LA INVESTIGACIÓN PARTICIPATIVA: MÉTODOS Y TÉCNICAS

CUENCA (ECUADOR): PYDLOS EDICIONES, 2015

ISBN: 978-9978-14-316-2

\author{
Liberto Carratalá Puertas \\ Universidad de Alicante, España \\ liberto.carratala@ua.es
}

\begin{abstract}
Cómo citar / Citation
Carratalá Puertas, Liberto (2017). "Crítica de libro: Francisco José Francés García, Antonio Alaminos Chica, Clemente Penalva Verdú y Óscar Antonio Santacreu Fernández: La investigación participativa: métodos y técnicas". OBETS. Revista de Ciencias Sociales, 12(Extra 1): pp-pp. 281-287. doi:10.14198/OBETS2017.12.1.21
\end{abstract}

Corren buenos tiempos para la participación ciudadana. Tanto es así que es frecuente encontrar en la prensa -principalmente local- alguna reseña breve acerca de prácticas en las que la ciudadanía es llamada, o ha estado implicada en alguna actividad que tiene fines consultivos o están vinculadas a las decisiones de un gobierno, por regla general también local.

No obstante, y dado el atractivo que la medida tiene para una parte importante de los representantes públicos, en muchas ocasiones bajo el paraguas de la participación ciudadana se consideran acciones institucionales que, desde un punto de vista metodológico y epistemológico, deberían clasificarse como meras consultas populares. Desde estas líneas, y tal y como apunta el contenido de este libro que se reseña, se advierte al lector o lectora de que la investigación participativa es un constructo metodológico de gran envergadura que no puede reducirse a la aplicación, sin ningún tipo de limitación, de una técnica o de varias. En realidad, es una metodología que tiene una ineludible vocación transformadora de la realidad. 
Precisamente, y por esta razón, la existencia de investigaciones participativas contribuye a alimentar el debate dentro de la comunidad científica sobre el papel de la investigación social. Mientras una parte de la misma considera que su papel debe limitarse a la descripción de los fenómenos sociales (como defendía Weber), la otra piensa que tiene que trascender el reducido espacio en el que habita el conocimiento para, a través de él, transformar el objeto de estudio, en realidad sujeto (caso de la tradición iniciada por los socialistas utópicos como Saint-Simon o Proudhon).

Con la intención de clarificar este panorama y también de servir como una herramienta útil a investigadores y estudiantes que quieren realizar sus primeras incursiones en el mundo de la participación ciudadana, un grupo de profesores, sociólogos, de la Universidad de Alicante ha publicado el libro La investigación participativa: métodos y técnicas. Esta obra forma parte de una colección de cinco monografías dedicadas a la metodología de investigación social. Han visto la luz fruto de la colaboración institucional entre el Programa Interdisciplinario de Población y Desarrollo Local Sustentable (PYDLOS) de la Universidad de Cuenca (Ecuador) y el Observatorio Europeo de Tendencias Sociales (OBETS), el cual está integrado en el Instituto Interuniversitario de Desarrollo Social y Paz de la Universidad de Alicante.

Esta colección de monografías metodológicas, entre la cual se encuentra la obra que se comenta en estas páginas, nació de un curso de formación de posgrado denominado "Investigación aplicada a las Ciencias Sociales: Técnicas de producción de datos y análisis" que se imparte en la citada ciudad ecuatoriana desde el año 2014.

Los autores de La investigación participativa: métodos y técnicas son consumados especialistas en la materia, todos ellos profesores del Departamento de Sociología II de la Universidad de Alicante. Francisco Francés García, quien encabeza la lista de coautores, cuenta con una dilatada trayectoria en el ámbito de la investigación participativa. Se formó como discípulo de uno de los padres de las metodologías participativas en España como es Tomás RodríguezVillasante. Ha escrito numerosas obras de referencia sobre esta temática y, actualmente, es consultor para distintos organismos nacionales e internacionales, prestando asesoramiento en diferentes ámbitos (salud, empleo, minorías étnicas, etc).

Completan la lista Antonio Alaminos Chica, es catedrático de Sociología Matemática. Dada su especialidad (Técnicas de Investigación Social), su participación en esta obra es un aval para dar el estatus que merece a estas metodologías de investigación; Clemente Penalva Verdú es profesor de "Análisis de Información Cualitativa" en el Grado de Sociología y de "Técnicas de Investiga- 
ción Social en Comunicación" en el Grado en Publicidad y Relaciones Públicas. Como veremos más adelante, materias estrechamente relacionados con las metodologías participativas; y Óscar Santacreu Fernández, profesor de "Análisis de Datos I" y "Sociología Matemática" en el Grado de Sociología y de la asignatura "Herramientas de Investigación Cuantitativa aplicadas a la Investigación”, en el Máster Universitario en Comunicación e Industrias Creativas, también con una vinculación íntima con la materia que se expone.

El título de la obra, por sí mismo, es bastante esclarecedor tanto sobre el contenido como en la intención de los autores de destacar la necesidad de contar con una guía para proceder con rigor científico en las prácticas en las que se pretende incluir a la ciudadanía.

El libro es fácil de leer, es directo y no se pierde en vaguedades de corte filosófico que puedan hacernos perder el hilo o dificultar la comprensión del método. De hecho, la estructura narrativa corresponde con la del diseño de una investigación participativa, lo cual facilita su comprensión y remarca el carácter procesual de esta metodología.

La obra está compuesta por siete capítulos. En el primero, el objetivo es ubicar la investigación participativa como exponente más destacado de la perspectiva dialéctica en el marco de la investigación social. Los autores citan pertinentemente como referente en esta discusión a Jesús Ibáñez, recordándonos que la investigación social es una tarea necesaria, pero a la vez imposible. Sin duda, el malogrado sociólogo español, tenía en mente a Weber cuando consideró que los datos se producen y no se recogen. Partiendo de esta lógica, podemos decir que la persona dedicada a la investigación no tiene más remedio que afectar a la realidad utilizando los instrumentos de medición que ha construido y analizarla con sus propios conceptos. El objeto de estudio, se convierte, de esta manera, en un problema que tiene un sentido y un significado que son sociales, inserto junto al investigador en unas coordenadas temporales y sociales que les dan forma. Ibáñez lo llamó el regreso del sujeto. No es, por tanto, descabellada la idea -y en esta ocasión contraria al juicio de Weberque, dado el carácter instrumental de la investigación, el científico pueda aspirar a transformar de manera intencional la realidad que le rodea, en tanto que somos sujetos de la investigación, al igual que los estudiados. Al fin y al cabo, un problema se investiga para intentar solucionarlo. A la luz de lo expuesto, los autores se basan en las aportaciones del propio Ibáñez y de otros referentes teóricos en el campo metodológico como Rodríguez-Villasante, Alberich y Bergua para elaborar una tipología (teniendo de nuevo a Weber presente) de la actividad investigadora en el ámbito social la cual resulta bastante útil para arrojar claridad sobre esta compleja cuestión. 
Para ello utilizan dos conceptos teóricos construidos por Ibáñez, que son, por una parte, los niveles tecnológico (con qué se hace la investigación), metodológico (cómo se hace la investigación) y epistemológico (para qué y para quién se investiga); y por la otra, las perspectivas distributiva (que tendría en la encuesta su principal exponente), estructural (representada por el grupo de discusión y la entrevista semiestructurada) y la dialéctica (dentro de la cual encontraríamos los socioanálisis, las metodologías IAP o los procesos participativos). De la combinación de los tres niveles y perspectivas obtenemos el paradigma de la investigación social vigente en la actualidad.

Una vez situada la investigación participativa dentro del paradigma, los autores se detienen, aunque de forma breve, en la importancia y el papel del sujeto en la investigación dialéctica, así como el método para articular la relación entre la teoría y la práctica. De esta forma, en los capítulos dos y tres los autores destacan que en la perspectiva dialéctica la relación sujeto-objeto pierde el interés. El fin deja de ser crear un relato de la realidad de manera pretendidamente objetiva. Por el contrario, gana fuerza conocer cómo se articulan las relaciones sociales en el medio instituido para provocar la transformación social. Una variación de las condiciones que vendría de la mano de procesos dialécticos de carácter asambleario. Así, nos encontramos, dicen los autores citando a Montañés, ante procesos que integran objetividades y subjetividades de forma compleja, multidimensional y dinámica.

En cuanto al método, teoría y práctica constituyen dos partes indivisibles en la investigación social. Los autores se apoyan nuevamente en RodríguezVillasante quien alerta que la realidad es un ente cada vez más complejo, con una evolución imprevisible e incierta. La teoría y la práctica se presentan como dos etapas "encabalgadas" donde la teoría está supeditada a la práctica y tiene como objetivo mejorarla continuamente. Esto supone una ruptura con el paradigma clásico de las ciencias en el que la teoría tiene una posición de superioridad jerárquica incuestionable tendente a acumular conocimiento. La consideración de la perspectiva dialéctica supone una inversión de las prioridades ya que, si el fin de la investigación social es la transformación de la realidad, el aparato teórico debe ponerse al servicio de este objetivo.

El cuarto capítulo recoge las características de la investigación participativa como fuerza generadora de procesos transformadores de la realidad, que es reflexiva y definida por los propios sujetos de la acción. Los autores indican con acierto que se conoce para implicar, que se implica para actuar y que se actúa para transformar y reflexionar sobre la acción. De esta manera, los procesos participativos constituyen un conjunto de posicionamientos y métodos aplicados que tienen como fin la transformación social a través de la acción y 
la reflexividad. Para alcanzarlos deben cumplir con cuatro tareas básicas: la difusión del proyecto (necesario para implicar a los sujetos), la formación (para proporcionar herramientas a los sujetos para que puedan diagnosticar su propia realidad), la consulta y la recogida de información (necesarios para retroalimentar el proceso y mantenerlo vivo) y la codecisión y la cogestión (implicarse significa hacerlo en el momento de tomar las decisiones y en el curso y orientación de los hechos derivados de las etapas anteriores).

Una vez definidas las etapas del proceso, los autores describen cuáles son los elementos estructurales y metodológicos de la acción participativa, a saber: componentes político-institucionales (los espacios que articulan las relaciones entre los representantes comunitarios y la ciudadanía), contextuales (los factores externos que determinan las relaciones entre los actores), operacionales (el conjunto de herramientas, técnicas, medios y mecanismos que facilitan el proceso participativo), y actitudinales (que determinan la disposición de los actores, a título individual y colectivo, a llegar a acuerdos y consensos sobre la realidad construida). No obstante, los autores advierten que el investigador tiene un margen de acción limitado en el que puede introducir estrategias metodológicas de forma eficaz, siendo esta posibilidad mayor en los componentes operacionales y actitudinales y menor en los contextuales y político-institucionales.

En el capítulo quinto los autores hacen referencia al enfoque InvestigaciónAcción-Participación (IAP) como el método de investigación participativa por excelencia. También se recoge las fases en las que se divide el proceso. No obstante, se advierte que una IAP es más un conjunto de posicionamientos metodológicos que un procedimiento unificado. Bajo esta premisa, los autores consideran que la IAP se caracteriza por unir la reflexión a la acción, por trascender la dicotomía objeto (investigado) - sujeto (investigador), que es sustituida por la relación sujeto-sujeto; por romper la asimetría de los actores de la comunidad (políticos, técnicos/expertos, ciudadanía); por intervenir en la génesis social de los fenómenos y no solo en sus síntomas o en sus efectos; por los objetivos de la investigación, que son definidos por las necesidades de la ciudadanía afectada por el problema; y por la finalidad del estudio, que siempre es la transformación de la situación-problema que afecta a la ciudadanía, entendiendo a la comunidad como el principal agente transformador.

Teniendo esto presente, los autores identifican tres fases que componen un ciclo de IAP: la fase de negociación de la demanda (con los actores sociales, el planteamiento del problema y los objetivos, la presentación y difusión del proceso, la recogida de información exploratoria, la autoformación, la constitución de un grupo dinamizador del proceso y el proyecto de IAP), el autodiagnóstico (proceso de devolución de la información, el trabajo de campo utilizan- 
do técnicas de creatividad social, el análisis y la interpretación de la información, la elaboración de un nuevo documento con el diagnóstico y las propuestas de acción) y la programación y puesta en marcha de acciones (talleres de negociación y decisión, propuestas de acción ciudadana, diseño del PAI, evaluación y la redacción del informe final). Una vez llegados a este punto, el informe debería servir para la reformulación del proceso con nuevos temas a tratar y la definición de nuevos objetivos. Los autores cierran este apartado destacando que este enfoque supone un avance en tres aspectos claves de la perspectiva dialéctica: la inclusividad, la intensidad y la influencia. Esta triada expresa el grado y calidad de la participación de una comunidad ya que hace referencia a quiénes participan, a la presencia o ausencia de espacios de deliberación previos a la toma de decisiones y al poder de la ciudadanía para hacerlas valer.

En el último capítulo, el más amplio de todos, los autores describen de qué partes se compone un proceso de participación ciudadana y qué técnicas de intervención serían las más adecuadas para cada una de sus fases. Se trata del apartado más práctico de toda la obra y una guía muy útil para aquellos y aquellas que quieran diseñar un proceso participativo incluyendo cómo van a intervenir en el nivel comunitario.

En su exposición, los autores han realizado una división de las técnicas a emplear en función de las etapas del proceso anteriormente señaladas. De esta forma, para la etapa correspondiente a la negociación inicial de objetivos y la construcción participada del proyecto se sugiere el uso del focus group, del sociograma, del mapa cognitivo y de la observación participante. Para el autodiagnóstico se recomiendan técnicas como las entrevistas o los grupos de discusión, el uso de técnicas biográficas, otras de tipo audiovisual como el Photovoice, el DAFO, la encuesta participativa, la encuesta deliberativa o el jurado ciudadano. Finalmente, para la programación e implementación de acciones se propone la técnica del árbol de problemas y soluciones, el flujograma situacional, los talleres de futuro y el método EASW (European Awareness Sustainability Workshop).

Todas ellas son herramientas que promueven la creatividad social basadas en la deliberación, la expresión de demandas y la racionalización de las mismas a través del debate y la reflexión colectiva. Tengo que destacar que los autores han descrito con precisión la implementación de cada una de estas técnicas. También han utilizado abundante material gráfico que sirven de valioso ejemplo para facilitar al investigador su puesta en práctica sobre el trabajo de campo.

Pocos peros se le pueden poner a este texto, cuyos autores han cuidado al máximo el lenguaje utilizado para facilitar la comprensión de lo que se presen- 
ta. Quizá, hubiera sido de agradecer un breve anticipo de las dificultades que se van a encontrar los investigadores en el momento de implementar estas técnicas. ¿La principal de todas? La dificultad de enganchar a la ciudadanía para participar en las actividades que se le proponen, poco habituada a involucrarse en iniciativas de este tipo. También, lo débil del compromiso de los representantes públicos para vincular las propuestas elaboradas por la ciudadanía a sus decisiones lo que redunda en el desencanto y descreimiento de los participantes en este tipo de procesos.

LIBERTO CARRATALÁ PUERTAS. Doctor en Sociología, actualmente trabaja como profesor en el Departamento de Sociología I de la Universidad de Alicante. Sus campos de investigación son la Sociología Política, las Políticas Públicas, los procesos de participación ciudadana, Demografía, Comunicación Política, jóvenes y valores humanos. Ha tomado parte en numerosas investigaciones para instituciones públicas donde ha trabajado como investigador principal y colaborador. Además, es autor de artículos, informes y libros sobre procesos de acción comunitaria.

Recibido: 10/10/2017 



\section{NORMAS EDITORIALES E INSTRUCCIONES PARA COLABORACIONES}

A continuación se ofrece un resumen de las normas editoriales e instrucciones para colaboraciones. Se recomienda consultar la última versión disponible en la dirección http://revistaobets.ua.es Instrucciones para el envío de originales

1. OBETS. Revista de Ciencias Sociales tiene como objetivo la difusión de estudios de naturaleza académica que permitan un mejor conocimiento de la sociedad contemporánea. En este sentido

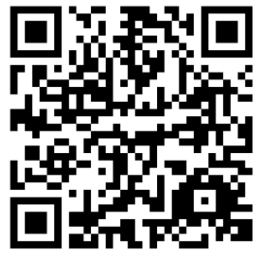
está abierta a la publicación de resultados de investigación empírica, metodológica o teórica de ámbito nacional e internacional, en un amplio abanico de temas inscritos en el ámbito de la Sociología y Ciencias Sociales, como estudios de opinión pública, metodología, demografía, estructura y cambio social, ciencia política, teoría sociológica, antropología o psicología social. Se dará prioridad a trabajos que comuniquen resultados de investigaciones académicas originales y que observen la estructura IMRyDC+B (introducción, metodología, resultados y discusión, conclusiones, y bibliografía actualizada).

2. Los trabajos, que podrán estar escritos en español, inglés, portugués o valenciano/ catalán deberán ser remitidos en formato digital, como archivo adjunto en formato .rtf o .doc a través de la página de envíos en la web de la revista. La recpción de artículos está abierta durante todo el año, a excepción del mes de agosto.

3. En cada edición se aceptan artículos que han sido positivamente evaluados al menos durante el mes anterior al de edición.

4. La extensión de los artículos que se remitan a la revista tendrán una extensión no superior a 10.000 palabras. Las notas de investigación (informe de hallazgos, aportaciones, etc.) tendrán una extensión máxima de 7.000 palabras. Las recensiones o críticas de libro no pueden superar las 3.000 palabras, y debe especificarse en ellas el autor, el título, la editorial, el lugar y la fecha de publicación de la obra objeto de recensión, así como el nombre, los apellidos y la dirección de contacto del autor de la crítica. Sólo se aceptarán recensiones de libros publicados en los últimos 3 años.

5. Los trabajos se enviarán en el siguiente formato

1. Tipo de letra Times New Roman, tamaño 12. Espacio sencillo, tamaño de página DIN A4.

2. Los diferentes apartados irán en negrita, en mayúsculas sólo la primera letra y con el mismo tipo y tamaño de fuente que el cuerpo del texto.

3. Los párrafos irán sangrados en la primera línea.

4. Las palabras en idioma diferente al original del trabajo deben ir en cursiva.

5. Las citas textuales deben ir entrecomilladas cuando sean inferiores a tres líneas. Si tienen más de 3 líneas irán sin entrecomillar y sangradas por el margen izquierdo, indicando en todo caso entre paréntesis el apellido del autor o autores del texto, el año de publicación y la página. Por ejemplo: (Ramírez, 2009: 10). Si hay más de dos autores pero menos de seis, se citarán todos ellos la primera vez y las veces siguientes se usará la expresión "et al.". Si son más de seis autores, se usará "et al." desde la primera mención. 
6. Para la bibliografía, al final del trabajo, deberá utilizarse el estilo APA (American Psychological Association). A continuación se ofrecen algunos ejemplos de formato:

\section{Libros y capítulos:}

Anwar, M. (1979). The Myth of Return: Pakistanis in Great Britain. London: Heinemann.

Esser, H. (2003). "What substance is there to the term 'Leitkultur'?". En R. Cuperus, K. A. Duffek y J. Kandel (Eds.), The challenge of diversity. European social democracy facing migration, integration and multiculturalism. Innsbrück/Vienna: Studienverlag, 47-58.

\section{Revistas:}

Berry, J. W. (1970). "Marginality, stress, and ethnic identification in an acculturated Aboriginal community". Journal of Cross-Cultural Psychology, 1, 17-22. Para documentos online, debe indicarse la fecha de consulta. Cuando se incluyan varias referencias de un mismo autor/a, es necesario indicar el nombre del autor en cada título y no usar guiones/rayas.

7. Las imágenes y diagramas se incluirán en el texto. En función de la resolución y calidad de dichas imágenes, la redacción puede solicitar el envío posterior de los archivos de imagen originales como condición para su publicación.

8. Los trabajos deben incluir, tanto en la lengua del artículo como en inglés, los siguientes elementos básicos: título, resumen de no más de 100 palabras, y entre 4 y 8 palabras clave separadas por punto y coma. Si el idioma del trabajo es el inglés, se incluirán estos elementos en inglés y en español. Para aquellos textos cuyo idioma sea diferente del inglés se debe enviar, además del abastract de 100 palabras, un abstract extendido en inglés del orden de 1000 a 1500 palabras en el que se describa con algo más de profundidad los métodos, datos, hallazgos e implicaciones del trabajo.

6. Los artículos han de ser inéditos. Dicha condición debe indicarse durante el proceso de envío al completar la lista de comprobación en la plataforma de la revista.

7. Las propuestas de contribuciones enviadas a la revista OBETS no pueden ser enviadas simultáneamente a ninguna otra publicación.

8. Todo original se somete al arbitraje por pares académicos. Con la finalidad de garantizar la imparcialidad en la selección de los artículos para publicar, se observa el anonimato tanto de autores como de evaluadores. Para proteger este anonimato, se respetará el siguiente formato en los trabajos enviados:

1. Una primera página en la que constará, únicamente, el título (en mayúsculas la primera letra y el resto en minúsculas), el nombre y apellidos de los autores, el centro de trabajo, dirección postal y correo electrónico, y teléfono y fax en su caso.

2. Una segunda página que contenga, nuevamente, el título, un resumen de no más de 100 palabras, y entre 4 y 8 palabras clave, separadas por punto y coma. Tanto el título como el resumen y las palabras clave deben aparecer en la lengua del trabajo y en inglés. 
3. El texto del trabajo (incluyendo el abstract extendido en inglés) en la página tercera y siguientes.

\section{Proceso de selección de originales}

1. Las obras recibidas pasarán por el siguiente proceso en el que cada paso requiere la superación del anterior:

1. Notificación de acuse de recibo.

2. Clasificación temática del artículo (UNESCO). Al menos dos miembros del Consejo Editorial comprobarán que el trabajo cumpla los estándares editoriales de un texto académico en el ámbito temático de la revista. Si hay al menos una valoración positiva, el trabajo pasará a la siguiente fase, y en caso contrario será notificado de manera justificada a los autores.

3. Revisión de aspectos formales y estilos, con notificación y devolución al autor en caso de existir errores.

4. Comprobación de la anonimización del artículo.

5. Revisión por dos personas evaluadoras externas, de acuerdo con criterios de

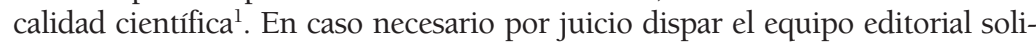
cita la contribución de un tercer evaluador/a. El formulario de evaluación está disponible en la web de la revista e incluye:

1. Evaluación de la originalidad y contribución científica.

2. Evaluación de la claridad y coherencia de objetivos, hipótesis, metodologías y conclusiones del trabajo.

3. Evaluación de la pertinencia y actualización de la bibliografía.

4. Evaluación de los aspectos formales (redacción, citas, organización del texto, tablas, gráficos, etc.)

5. Recomendación final sobre el trabajo y, en su caso, sugerencias de modificación o razones para no publicación.

6. Envío al autor del informe de revisión con la decisión correspondiente (aceptación, aceptación pendiente de rectificaciones, rechazo) de forma motivada en un plazo máximo de 6 meses desde la recepción del artículo.

2. En el caso de que un artículo sea aceptado para su publicación, se solicitará al autor/a la firma de un documento de cesión de derechos a fin de que sea publicado en la revista bajo licencia Creative Commons Reconocimiento 4.0 España (CC BY 4.0). De igual modo, las pruebas de imprenta serán enviadas al autor/a en formato PDF cuando estén disponibles y deberán ser devueltas a la secretaría de la revista en el plazo máximo de una semana, con las oportunas correcciones.

3. El proceso de evaluación suele tomar unos 4-6 meses. Al tratarse de una revista semestral, una vez aceptado un artículo la publicación final puede llevar por lo general hasta 6 meses más.

4. Para cualquier duda o aclaración, puede mandar un correo electrónico a la siguiente dirección: revistaobets@ua.es

\footnotetext{
${ }^{1}$ Centro de Información y Documentación Científica (CINDOC) CSIC. Madrid, 2001.
} 


\section{Principios éticos de publicación}

La publicación de artículos en una revista con revisión por pares es un reflejo directo de la calidad del trabajo de sus autores, y del compromiso y cualificación de los investigadores que actúan como revisores. Por ello OBETS. Revista de Ciencias Sociales es una publicación comprometida con los principios éticos de la actividad científica en los siguientes términos:

\section{Publicación y autoría}

Todos los artículos deben incluir un listado de referencias, así como indicar si han recibido apoyo económico. Los trabajos deben estar libres de plagio o fraude científico, cuyos supuestos ${ }^{2}$ se enumeran de manera no exhaustiva a continuación:

- Plagio: copia literal sin entrecomillar y citar la fuente; copia sustancial (materiales de investigación, procesos, tablas...); parafrasear o reproducir ideas sin citar la fuente y/o cambiando el significado original; reutilizar y enviar textos propios ya publicados sin indicar la fuente y el parafraseo abusivo incluso citando la fuente.

- Fraude científico: no reconocimiento de todos los investigadores/as participantes en la elaboración del trabajo, el envío simultáneo a varias publicaciones, la división de un trabajo en partes diferentes que comparten las mismas hipótesis, población y métodos, así como la utilización de datos falsos o no probados. Finalmente, los autores/as deben declarar a la revista los potenciales conflictos de interés cuando envían un trabajo.

\section{Responsabilidad de los autores}

- El envío de trabajos a OBETS supone la lectura y aceptación de las normas editoriales y de publicación de la revista, incluida la participación en un proceso anónimo de evaluación por pares.

- Todos los autores que firman un trabajo deben haber contribuido de manera significativa a su elaboración y deben estar de acuerdo con el resultado final y con el envío del trabajo para su evaluación.

- Los trabajos deben reconocer a todos los autores que han participado en su elaboración.

- Los datos utilizados en el artículo deben ser reales y auténticos.

- Los autores asumen la obligación de corregir y/o retractarse ante posibles errores detectados posteriormente.

- Los artículos han de ser inéditos y no pueden ser enviados simultáneamente a ninguna otra publicación.

\section{Proceso de revisión}

Todos los artículos enviados a la revista se someten a un proceso de revisión por pares con las siguientes características:

- La selección de los revisores se realiza en función de normas y principios previos basados tanto en su cualificación como en la calidad de su producción científica.

\footnotetext{
${ }^{2}$ Fuente: http://www.ethics.elsevier.com/
} 
- El proceso de revisión será totalmente anónimo tanto para los autores como para los revisores. Los artículos y sus revisiones serán tratados confidencialmente.

- Los revisores consideran, entre sus criterios de evaluación, el respeto a los principios éticos esenciales en la investigación científica.

- Los juicios expresados en las revisiones deben ser objetivos.

- Tanto autores como revisores deben revelar las relaciones y fuentes de financiación que puedan generar potenciales conflictos de intereses.

\section{Responsabilidades de los editores}

- El equipo editorial tiene la responsabilidad y autoridad para aceptar o rechazar un artículo basándose en las revisiones.

- El equipo editorial revelará en su caso las relaciones o fuentes de financiación que puedan ser potencialmente consideradas como conflictos de intereses respecto a los artículos que rechaza o acepta.

- Sólo se aceptarán los artículos en los que existe una evidencia cierta sobre el cumplimiento de las normas editoriales

- El Consejo de Redacción de OBETS. Revista de Ciencias Sociales se reserva el derecho de retirar cualquier trabajo recibido, aceptado o ya publicado en caso de constatarse plagio, falsificación o publicación duplicada, así como los diversos supuestos de fraude científico anteriormente enumerados. Del mismo modo, promoverá la publicación de correcciones o retractaciones frente a errores detectados.

- El equipo editorial se compromete a preservar el anonimato de los revisores de manera que nunca puedan asociarse con los artículos revisados.

\section{Cuestiones éticas de publicación}

El equipo editorial se compromete a:

- Vigilar y preservar los principios éticos de publicación

- Mantener la integridad del expediente académico

- Evitar la publicación de material plagiado o elaborado de manera fraudulenta

- Estar abierto a la publicación de correcciones, clarificaciones, retractaciones y disculpas siempre que sea necesario

- Ofrecer apoyo en el proceso de retractación de artículos

- Realizar todas las acciones necesarias para cumplir los estándares de compromiso intelectual y ético

\section{Aviso legal}

A efectos de lo estipulado en los artículos 138-143 de la Ley de Propiedad Intelectual, la publicación de un trabajo que atente contra dichos derechos será responsabilidad del autor/a. El equipo editorial de OBETS. Revista de Ciencias Sociales no se hace responsable, en ningún caso, de la credibilidad y autenticidad de los trabajos. Del mismo modo, las opiniones y hechos expresados en cada artículo son de exclusiva responsabilidad de sus autores/as y OBETS. Revista de Ciencias Sociales no se identifica necesariamente con ellos. Los autores conservan el copyright de su artículo sin restricciones. 



\section{EDITORIAL STANDARDS AND INSTRUCTIONS FOR CONTRIBUTORS}

Summary of guidelines for submitting manuscripts. Please check the latest version in http://revistaobets.ua.es

\section{Guidelines for submitting manuscripts}

1. The journal OBETS. Revista de Ciencias Sociales (OBETS. Journal of Social Sciences) aims at the dissemination of academic nature studies that allow a better understanding of contemporary society. In this sense it is open to the publication of results of empi-

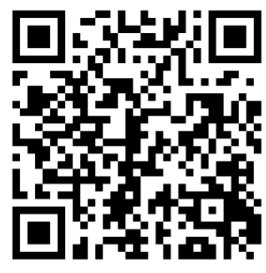
rical, methodological or theoretical research at national and international level, in a wide range of subjects enrolled in the field of sociology and social sciences, as public opinion surveys, methodology, demographics, structure and social change, political science, sociological theory, anthropology or social psychology. Priority will be given to works that communicate results of original academic research and to use the IMR\&DC+B structure (introduction, methods, results and discussion, conclusions, and updated bibliography).

2. Papers may be written in English, Italian, Portuguese, Spanish or Valencian/Catalan. They must be submitted in digital format as an attachment, in .Rtf or .Doc format through the submission page on the website of OBETS Journal. Receipt of items is open all the the year, except August.

3. For each edition are accepted only articles that have been positively evaluated at least during the month prior to the edit month.

4. The length of papers should not exceed 10000 words. The research notes (report on findings, contributions, etc.) should not exceed 7000 words. The book reviews cannot exceed 3000 words, and should include the author, title, publisher, place and date of publication of the book. The full name and address of Contact of the author of the critique must also be included. We only accept reviews of books published in the last 3 years.

5. The works will be sent in the following format

1. Font Times New Roman, font size 12. Single space, page size A4.

2. Section headers in bold. Capitalize only the first letter, and with the same type and font size that the text body.

3. Paragraphs indented on the first line.

4. The words in a language other than the original work in italics.

5. Textual citations must be quoted if they are less than three lines. If you have more than three lines shall be indented by the left margin without quotation marks. In any case, indicate in parentheses the surname of the author or authors of the text, the year of publication and page. For example: (Ramírez, 2009: 10). If there are more than two authors but less than six, all of them will be cited the first time and the time following use the expression "et al.". If more than six authors, use "et al." from the first mention. 
6. The bibliography at the end of the work shall use APA style (American Psychological Association). Please find below some examples of format:

Books and chapters:

Anwar, M. (1979). The Myth of Return: Pakistanis in Great Britain. London: Heinemann.

Esser, H. (2003). "What Is There Substance to the term 'Leitkultur'?". In R. Cuperus, KA Duffek and J. Kandel (Eds.), The challenge of diversity. European Social Democracy Facing Migration, integration and multiculturalism. Innsbruck / Vienna: Studienverlag, 47-58.

Journals:

Berry, J. W. (1970). "Marginality, stress, and ethnic identification in an acculturated Aboriginal community". Journal of Cross-Cultural Psychology, 1, 17-22.

For documents online, you must indicate the accessed date.

When several references of a same author are included, it is necessary to indicate the name of the author in each title instead of using hyphens/dashes.

7. Pictures and diagrams should be included in the text. Depending on the resolution and quality of the images, the Journal can ask for the original image files as a condition for the publication.

8. Papers should include the following basic elements, both in the language of the article and in English: title, an abstrat no longer than 100 words, and between 4 and 8 keywords separated by semi-coma. If the working language is English, these elements should be included in English and Spanish. If the paper is written in a language other than English, in addition to the 100 words abstract, an extended English abstract must be included, on the order of 1000-1500 words, going into some depth about the papers methods, data, findings and implications.

6. Articles must be unpublished. That condition should be declared during the sending process, when completing the checklist in the Journal webside.

7. Proposals for contributions sent to the OBETS journal may not be sent simultaneously to any other publication.

8. All original is submitted to arbitration by academic peers. In order to ensure impartiality in the selection of articles to publish, the journal observes the anonymity of authors and evaluators. To protect the anonymity, please respect the following format in contributions:

1. A first page that contain only the title (capitalize the first letter and the rest in lower case), the name of the authors, the workplace, and email address, telephone and fax.

2. A second page containing, again, the title (but not the name of author/s), an abstract no longer than 100 words, and between 4 and 8 keywords, separated by semi-coma. Title, abstract and keywords should appear in the original language and in English. 
3. The text of the work (including the extended English abstract) will follow in the third and following pages.

\section{Selection process of manuscripts}

1. The works received will go through the following process in which each step requires overcoming previous

\section{Acknowledgement Notice}

2. Subject classification of the article (UNESCO). At least two members of the Editorial Board will check the compliance of the article with editorial standards of an academic text in the subject area of the journal. If there is at least a positive assessment, the article proceeds to the next phase; otherwise it will be justifiably notified to authors.

3. Review of formal aspects and styles, with notice and return to the author in case of errors.

4. Verification of the anonymization of the article

5. Review by two external evaluators, according to scientific quality criteria ${ }^{1}$. If necessary by disparate evaluations, editorial team requests the contribution of a third evaluator. The evaluation form (available on the website of the journal) includes:

1. Evaluation of the originality and scientific contribution.

2. Evaluation of the clarity and consistency of objectives, hypothesis, methodologies and conclusions.

3. Evaluation of the adequacy and updating of the bibliography.

4. Evaluation of the formal aspects (writing style, quotes, text organization, Tables, graphics, etc).

5. Final recommendation on the work and, where appropriate, suggestions for change or reasons for non-publication.

6. The author(s) will be notified with the relevant decision (acceptance, acceptance pending revisions, reject) on a reasoned way within a maximum period of 6 months.

2. When an article is accepted for publication, the author will be asked to sign a transfer of rights document in order to allow the journal to publish the article under a Creative Commons Attribution 4.0 Spain license (CC BY 4.0). Similarly, proofs will be sent to the author in PDF format when they are available and they must be returned to the secretariat of the journal within one week, with appropriate corrections.

3. The evaluation process is expected to last about 4-6 months. Since Obets Journal is semmianual, the final publication of the article once accepted usually can take up to 6 more months.

4. For any questions or doubts, please send an email to the following address: revistaobets@ua.es

${ }^{1}$ Centre for Scientific Information and Documentation (CINDOC) CSIC. Madrid, 2001. 


\section{Publication ethics and malpractice statement}

The publication of articles in a peer-review journal is a direct reflection of the quality of the work of their authors, and the commitment and qualifications of the researchers who act as reviewers. Therefore OBETS. Revista de Ciencias Sociales is a publication committed to the ethical principles of scientific activity in the following terms:

\section{Publication and authorship}

All manuscripts must include a list of references, and indicate whether they have received financial support. Works must be free of plagiarism or scientific fraud. Illustrative cases ${ }^{2}$ of plagiarism and scientific fraud can be consulted in a non-exhaustive list below:

- Plagiarism: literal copy without quoting and referencing the source; substantial copying (research materials, processes, tables...); paraphrasing or reproducing ideas without citing the source and/or changing the original meaning; text-recycling (reusing a published own text) without indicating the source, and abusive paraphrasing even quoting the source.

- Scientific fraud: no recognition of all the participating researchers in the study, simultaneous submission to several publications, the division of a work in different parts ('slices') that share the same hypotheses, population and methods, as well as the use of false or unproven data. Finally, the authors should disclose potential conflicts of interest to the journal when a manuscript is sent.

2. Responsibility of the authors

- The manuscripts submission to OBETS involves reading and acceptance of the journal publishing guidelines, including participation in an anonymous peerreview process.

- All authors signing a work must have contributed significantly to its development and must agree both with the end result and with the manuscript submission for evaluation.

- Manuscripts must acknowledge all authors who have participated in their elaboration.

- Data used in the article must be real and authentic

- The authors assume the obligation to retract/correct when possible errors are later detected.

- Articles must be original and cannot be sent simultaneously to any other publication.

\section{Review Process}

All articles submitted to the journal are subjected to a peer review process with the following characteristics:

- The selection of reviewers is done according to rules and principles based on both their qualification and the quality of their scientific production.

- The review process will be totally anonymous both for authors and for reviewers. Manuscripts and reviews will be treated confidentially.

\footnotetext{
${ }^{2}$ Source: http://www.ethics.elsevier.com/
} 
- Reviewers will take into account for their evaluation criteria the respect for the ethical principles that are essential in scientific research.

- The judgments expressed in the reviews should be objective.

- Authors and reviewers should disclose all relationships and funding sources that could generate potential conflicts of interest.

\section{Responsibilities of Editors}

- The editorial board has the responsibility and authority to accept or reject a manuscript based on the peer reviews.

- The editorial board will reveal any relationships or funding sources that could potentially be considered conflicts of interest regarding the rejection or the acceptance of manuscripts.

- The journal only accepts manuscripts when reasonably certain of compliance with editorial standards.

- The Editorial Team reserves the right to remove any received, accepted or already published work where plagiarism, falsification, duplicate publication or scientific fraud is found. Similarly, it will encourage the publication of retractions or corrections to errors detected.

- The editorial team is committed to preserve the anonymity of the reviewers so that they can never be associated with the reviewed manuscripts.

\section{Publishing ethical Issues}

The editorial board is committed to:

- Monitoring and maintaining the publishing ethics

- Maintaining the integrity of the academic record

- Avoid publishing plagiarized or fraudulently prepared material

- Be willing to publish corrections, clarifications, retractions and apologies when needed

- Provide support in the process of retracting articles

- Perform all actions required to meet the standards of intellectual and ethical commitment

\section{Disclaimer}

Regarding the provisions in articles 138-143 of the Spanish Law on Intellectual Property, the publication of a work prejudicial to those rights shall be the responsibility of the author. The editorial team of OBETS. Revista de Ciencias Sociales is not responsible, in any case, for the credibility and authenticity of the works. In the same way, the opinions and facts expressed in each article are the sole responsibility of the authors and OBETS. Revista de Ciencias Sociales does not necessarily agree with them. The authors hold the copyright of their articles without restrictions. 
